

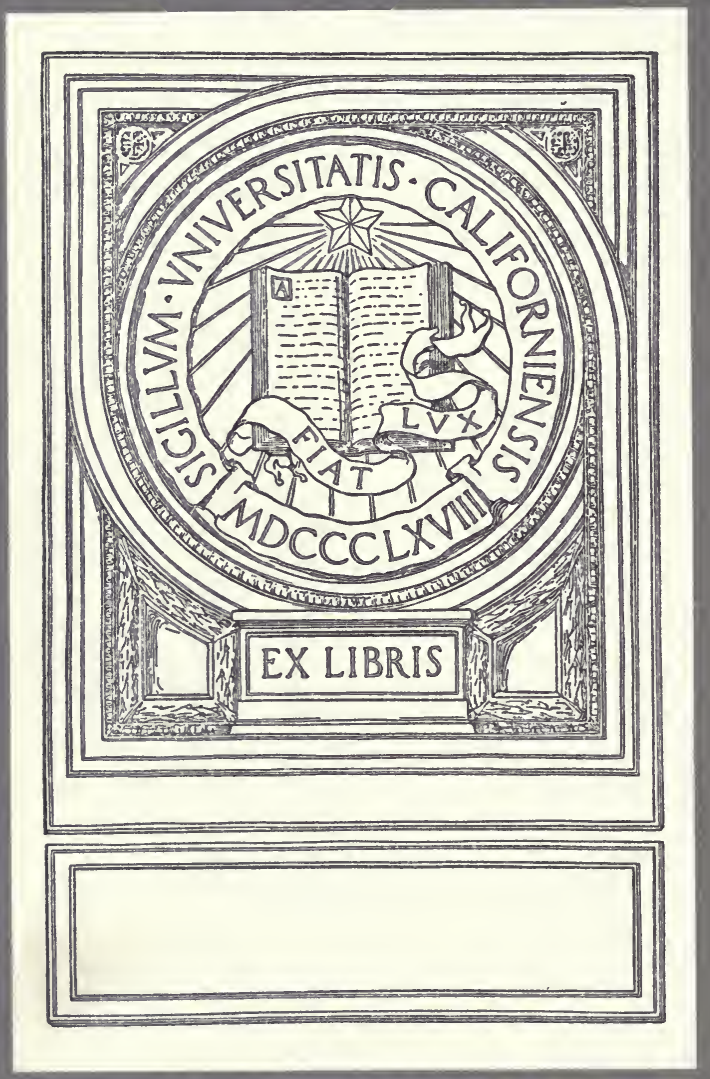






$$
\text { - }
$$




\title{
THE
}

\section{DYNAMICAL THEORY}

\author{
$\mathrm{OF}$ \\ SOUND
}

BY

HORACE LAMB, Sc.D., LL.D., F.R.S.

PROFESSOR OF MATHEMATICS IN THE VICTORIA UNIVERSITY OF MANCHESTER; FORMERLY FELLOW OF TRINITY COLLEGE, CAMBRIDGE

\author{
LONDON \\ EDWARD ARNOLD
}

1910

[All Rights reserved] 
$\theta 1224$

L3

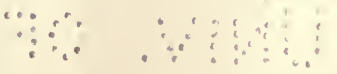

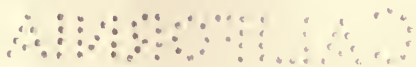




\section{PREFACE}

A COMPLETE survey of the theory of sound would lead into The many fields, physical, physiological, psychological, aesthetic. devoted mainly to the dynamical aspect of the subject. It is accordingly to a great extent mathematical, but I have tried to restrict myself to methods and processes which shall be as simple and direct as is possible, regard being had to the nature of the questions treated. I hope therefore that the book may fairly be described as elementary, and that it may serve as a stepping stone to the study of the writings of Helmholtz and Lord Rayleigh, to which I am myself indebted for almost all that I know of the subject.

The limitation of methods has involved some sacrifices. Various topics of interest have had to be omitted, whilst others are treated only in outline, but I trust that enough remains to afford a connected view of the subject in at all events its more important branches. In the latter part of the book a number of questions arise which it is hardly possible to deal with according to the stricter canons even of mathematical physics. Some recourse to intuitional assumptions is inevitable, and if in order to bring such questions within the scope of this treatise I have occasionally carried this license a little further than is customary, I would plead that this is not altogether a defect, since attention is thereby concentrated on those features which are most important from the physical point of view. 
Although a few historical notes are inserted here and there, there is no attempt at systematic citation of authorities. The reader who wishes to carry the matter further will naturally turn in the first instance to Lord Rayleigh's treatise, where full references, together with valuable critical discussions, will be found. I may perhaps be allowed to refer also to the article entitled "Schwingungen elastischer Systeme, insbesondere Akustik," in the fourth volume of the Encyclopädie der mathematischen Wissenschaften (Leipzig, 1906).

I have regarded the detailed description of experimental methods as lying outside my province. I trust, however, that no one will approach the study of the subject as here treated without some first-hand acquaintance with the leading phenomena. Fortunately, a good deal can be accomplished in this way with very simple and easily accessible appliances; and there is, moreover, no want of excellent practical manuals.

I have to thank Mr H. J. Priestley for kind assistance in reading the proof-sheets.

H. L.

January, 1910. 


\section{CONTENTS}

\section{INTRODUCTION}

ART.

1. Simple Vibrations and Pure Tones . . . . . 1

2. Musical Notes . . . . . . . . 3

3. Musical Intervals. Diatonic Scale . . . . . 5

\section{CHAPTER I}

\section{THEORY OF VIBRATIONS}

4. The Pendulum . . . . . . . . . 8

5. Simple-Harmonic Motion . . . . . . . . 9

6. Further Examples . . . . . . . . . 11

7. Dynamics of a System with One Degree of Freedom. Free Oscillations . . . . . . . . . . 12

8. Forced Oscillations of a Pendulum . . . . . 16

9. Forced Oscillations in any System with One Degree of Freedom. Selective Resonance . . . . . 20

10. Superposition of Simple Vibrations . . . . . . 22

11. Free Oscillations with Friction . . . . . . 24

12. General Dissipative System with One Degree of Freedom. Effect of Periodic Disturbing Forces . . . . 27

13. Effect of Damping on Resonance. . . . . . . 32

14. Systems of Multiple Freedom. Examples. The Double Pendulum . . . . . . . . . . . 34

15. General Equations of a Multiple System . . . . 41

16. Free Periods of a Multiple System. Stationary Property . 44

17. Forced Oscillations of a Multiple System. Principle of Reciprocity . . . . . . . . . 47

18. Composition of Simple-Harmonic Vibrations in Different Directions . . . . . . . . . . 48

19. Transition to Continuous Systems . . . . $\quad 52$

20. On the Use of Imaginary Quantities . . . . . $\quad 53$

21. Historical Note . . . . . . . . . 58 


\section{CHAPTER II}

\section{STRINGS}

ART.

22. Equation of Motion. Energy . . . . . . $\quad$. 59

23. Waves on an Unlimited String . . . . . . 61

24. Reflection. Periodic Motion of a Finite String . . . 64

25. Normal Modes of Finite String. Harmonics . . . 68

26. String excited by Plucking, or by Impact . . . . 72

27. Vibrations of a Violin String . . . . . . . 75

28. Forced Vibrations of a String . . . . . . $\quad$. 80

29. Qualifications to the Theory of Strings . . . . 81

30. Vibrations of a Loaded String . . . . . . 82

31. Hanging Chain . . . . . . . . 84

\section{CHAPTER III}

\section{FOURIER'S THEOREM}

32. The Sine-Series . . . . . . . . . 87

33. The Cosine-Series . . . . . . . . 92

34. Complete Form of Fourier's Theorem. Discontinuities . 92

35. Law of Convergence of Coefficients . . . . $\quad$. 94

36. Physical Approximation. Case of Plucked String . $\quad 96$

37. Application to Violin String . . . . . . 98

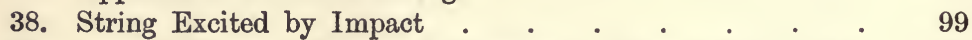

39. General Theory of Normal Functions. Harmonic Analysis 101

\section{CHAPTER IV}

\section{BARS}

40. Elementary Theory of Elasticity. Strains . . . 106

41. Stresses . . . . . . . . . 108

42. Elastic Constants. Potential Energy of Deformation . $\quad 110$

43. Longitudinal Vibrations of Bars . . . . . . 114

44. Plane Waves in an Elastic Medium . . . . . 118

45. Flexural Vibrations of a Bar . . . . . . 120

46. Free-free Bar . . . . . . . . . . . 124

47. Clamped-free Bar . . . . . . . . . . . 127

48. Summary of Results. Forced Vibrations . . . . 130

49. Applications . . . . . . . . . . . . 131

50. Effect of Permanent Tension . . . . . . 132

51. Vibrations of a Ring. Flexural and Extensional Modes . 133 


\section{CHAPTER V \\ MEMBRANES AND PLATES}

ART.

52. Equation of Motion of a Membrane. Energy . . $\quad 139$

53. Square Membrane. Normal Modes . . . . $\quad 142$

54. Circular Membrane. Normal Modes . . . . . 144

55. Uniform Flexure of a Plate . . . . . . . 150

56. Vibrations of a Plate. General Results . . . . 152

57. Vibrations of Curved Shells . . . . . . . . 155

\section{CHAPTER VI}

\section{PLANE WAVES OF SOUND}

58. Elasticity of Gases

59. Plane Waves. Velocity of Sound . . . . . 160

60. Energy of Sound-Waves . . . . . . . . . 163

61. Reflection . . . . . . . . . . . 168

62. Vibrations of a Column of Air . . . . . . 170

63. Waves of Finite Amplitude . . . . . . . 174

64. Viscosity . . . . . . . . . . . 183

65. Effect of Heat Conduction . . . . . . . 187

66. Damping of Waves in Narrow Tubes and Crevices . 190

\section{CHAPTER VII}

\section{GENERAL THEORY OF SOUND WAVES}

67. Definitions. Flux. Divergence . . . . . . 197

68. Equations of Motion . . . . . . . . 200

69. Velocity-Potential . . . . . . . . . 201

70. General Equation of Sound Waves . . . . . 204

71. Spherical Waves . . . . . . . 205

72. Waves resulting from a given Initial Disturbance . . 212

73. Sources of Sound. Reflection . . . . . . 214

74. Refraction due to Variation of Temperature . . $\quad 216$

75. Refraction by Wind . . . . . . . 219

\section{CHAPTER VIII}

\section{SIMPLE-HARMONIC WAVES. DIFFRACTION}

76. Spherical Waves. Point-Sources of Sound . . . . 223

77. Vibrating Sphere . . . . . . . . . . 228

78. Effect of a Local Periodic Force . . . . . . 233 
ART.

79. Waves generated by Vibrating Solid . . . . . 236

80. Communication of Vibrations to a Gas . . . . 237

81. Scattering of Sound Waves by an Obstacle . . . 240

82. Transmission of Sound by an Aperture . . . . 244

83. Contrast between Diffraction Effects in Sound and Light. Influence of Wave-Length . . . . . . 248

\section{CHAPTER IX}

\section{PIPES AND RESONATORS}

84. Normal Modes of Rectangular and Spherical Vessels . $\quad 254$

85. Vibrations in a Cylindrical Vessel . . . . . $\quad$. 259

86. Free Vibrations of a Resonator. Dissipation . . . 260

87. Corrected Theory of the Organ Pipe . . . . . $\quad 266$

88. Resonator under Influence of External Source. Reaction on the Source . . . . . . . . . 270

89. Mode of Action of an Organ Pipe. Vibrations caused by

90. Theory of Reed-Pipes . . . . . . . . 278

\section{CHAPTER $\mathrm{X}$}

\section{PHYSIOLOGICAL ACOUSTICS}

91. Analysis of Sound Sensutions. Musical Notes . . . 284

92. Influence of Overtones on Quality . • • . $\quad 286$

93. Interference of Pure Tones. Influence on the Definition of Intervals . $\quad . \quad$. $\quad . \quad$. . . 287

94. Helmholtz Theory of Audition . . . . . 289

95. Combination-Tones . . . . . . . . 292

96. Influence of Combination-Tones on Musical Intervals • 297

97. Perception of Direction of Sound. . . . . . 298

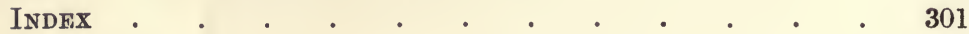




\section{THE DYNAMICAL THEORY OF SOUND}

\section{INTRODUCTION}

\section{Simple Vibrations and Pure Tones.}

In any ordinary phenomenon of sound we are concerned, first with the vibrating body, e.g. a string or a tuning fork or a column of air, in which the disturbance originates, secondly with the transmission of the vibrations through the aerial medium, next with the sensations which the impact of the waves on the drum of the ear somehow and indirectly produces, and finally with the interpretation which, guided mainly and perhaps altogether by experience, we put upon these sensations. It is in something like this natural order that the subject will be discussed in the following pages, but the later stages involving physiological and psychological questions can only be touched upon very lightly.

As few readers are likely to take up this book without some previous knowledge of the subject we may briefly recapitulate a few points which will be more or less familiar, with the view of fixing the meaning of some technical terms which will be of constant occurrence. Many of the matters here referred to will of course be dealt with more fully later.

The frontier between physics and physiology is reached at the tympanic membrane, and from the physical standpoint it is to the variations of pressure in the external ear-cavity that we must in the last resort look, under normal (as distinguished from pathological) conditions, for the cause of whatever sensations of sound we experience. These variations may conveniently be imagined to be exhibited graphically, like the ordinary variations of barometric pressure, by a curve in which the abscissae represent times and the ordinates deviations of the 
pressure on one side or other of the mean, the only difference being that the horizontal and vertical scales are now enormously magnified.

The variety of such curves is of course endless, and it is impossible to suppose that a distinct provision is made in the ear for the recognition of each, or even of each of the numerous classes into which they might conceivably be grouped. It is therefore necessary to analyse, as far as possible, both the vibration-forms and the resulting sensations into simpler elements which shall correspond each to each.

As regards the vibration-forms, there is one mode of resolution which at once claims consideration on dynamical grounds. The fundamental type of vibration in Mechanics is that known as "simple-harmonic," which is represented graphically by a curve of sines (Fig. 3, p. 10). This is met with in the pendulum, and in all other cases of a freely vibrating body or mechanical system having only one degree of freedom. It can moreover be shewn that the most complicated oscillation of any system whatever may, so far as friction can be neglected, be regarded as made up of a series of vibrations of this kind, each of which might be excited separately by suitable precautions. The reason for the preeminent position which the simpleharmonic type occupies in Mechanics is that it is the only type which retains its character absolutely unchanged whenever it is transmitted from one system to another. This will be explained more fully in the following chapter.

The analysis of sensations is a much more delicate matter, and it was a great step in Acoustics when $\mathrm{Ohm}^{*}$ in 1843 definitely propounded the doctrine that the simplest and fundamental type of sound-sensation is that which corresponds to a simple-harmonic vibration. This implies that all other sound-sensations are in reality complex, being made up of elementary sensations corresponding to the various simpleharmonic constituents into which the vibration-form can be resolved. The statement is subject to some qualifications, in particular as to the degree of independence of elementary

* G. S. Ohm (1787-1854), professor of physics at Munich 1849-54, known also as the author of "Ohm's Law" of electric conduction. 
sensations very near to one another in the scale, but these need not detain us at present. It may be regarded as in the main fully established, chiefly in consequence of the labours of Helmholtz*. The sensation corresponding to a simple-harmonic vibration is called a "simple tone" or a "pure tone," or merely a "tone." The sound emitted by a tuning fork fitted with a suitable resonator, or by a wide stopped organ pipe, gives the best approach to it.

Since the form of the vibration-curve is fixed, the distinction between one simple tone and another can only be due to difference of frequency or of amplitude. The "frequency," i.e. the number of complete vibrations per second, determines the "pitch," greater frequency corresponding to higher pitch. The lower and upper limits of frequency for tones audible to the human ear are put at about 24 and 24,000 respectively; the range employed in music is much narrower, and extends only from about 40 to 4000 . As between tones of the same pitch, the amplitude, or rather its square, determines the rate of supply of energy to the ear and so the relative "intensity," but it will be understood that it is physical rather than subjective intensity that is here involved. Between tones of different pitch only a vague comparison of loudness is possible, and this may have little relation to the supply of energy. Near the limits of audibility the sensation may be feeble, even though the energy-supply be relatively considerable.

\section{Musical Notes.}

From the chaos of more complex sounds there stands out a special class, viz. that of musical "notes." The characteristic of such sounds is that the sensation is smooth, continuous, and capable (at least in imagination) of indefinite prolongation without perceptible change. The nature of the corresponding vibrations is well ascertained. If we investigate any contrivance

* Hermann Helmholtz (1821-94), successively professor of physiology (Königsberg 1849), anatomy (Bonn 1855), physiology (Heidelberg 1858) and physics (Berlin 1871). Reference will often be made to his classical work : Die Lehre von den Tonempfindungen als physiologische Grundlage fur die Theorie der Musik, Brunswick, 1862. An English translation from the third edition (1870) was published by A. J. Ellis under the title Sensations of Tone, London, 1875. 
by which a note of good musical quality is actually produced, we find that the vibration can be resolved into a series of simpleharmonic components whose frequencies stand to one another in a certain special relation, viz. they are proportional to the numbers $1,2,3, \ldots$ Individual members of the series may be absent, and there is practically a limit on the ascending side, but no other ratios are admissible. It is evident from the above relation that the resultant vibration-form is necessarily periodic in character, recurring exactly at intervals equal to the period in which the first member of the series goes through its phases. It must be remembered, however, that the ear has no knowledge of the periodic character as such, and it must not be supposed that every periodic vibration will necessarily produce a sensation which is musically tolerable. The superposition of simple-harmonic vibrations to produce periodic vibration-forms is illustrated by some of the diagrams given below in Chapter III.

One musical note may differ from another in respect of pitch, quality, and loudness. The pitch is usually estimated as that of the first simple-harmonic vibration in the series, viz. that of lowest frequency, but if the amplitude of this first component be relatively small, and especially if it fall near the lower limit of the audible scale, the estimated pitch may be that of the second component.

By "quality" is meant that unmistakable character which distinguishes a note on one instrument from the note of the same pitch as given by another. Every musical instrument has as a rule its own specific quality*, which is seldom likely to be confused with that of another. Everyone recognizes for instance the difference in character between the sound of a flute, a violin, a trumpet, and the human voice, respectively. It is obvious that difference of quality, so far as it is not due to adventitious circumstancest, can only be ascribed to difference of vibration-form, and so to differences in the relative amplitudes and phases of the simple-harmonic constituents. According to

* French timbre; German Klangfarbe.

+ Such as the manner in which the sound sets in and ceases; this is different for instance in the violin and the piano. 
Helmholtz the influence of phase is inappreciable. This has been contested by some writers, but there can be no doubt that in most cases the difference of quality is a question of relative amplitudes alone.

Comparisons of loudness can only be made strictly between sounds of the same quality and about the same pitch.

It follows from the preceding that, so far as Ohm's law is valid, the sensation of a musical note must be complex, and made up of the simpler sensations, or tones, which correspond to the various simple-harmonic elements in the vibration-form. This doctrine has to contend with strong and to some extent instinctive prepossessions to the contrary, and some preliminary training is usually necessary before it is accepted as a fact of personal experience. We shall return to this question later; at present we merely record that that element in the sensation which corresponds to the gravest simple-harmonic constituent is called the "fundamental tone," and that the others are termed its " overtones" or " harmonics."

\section{Musical Intervals. Diatonic Scale.}

There are certain special relations, familiar to trained ears, in which two notes or two simple tones may stand to one another. These are the various consonant and other "intervals." Physically they are marked by the property that the frequencies corresponding to the respective pitches are in a definite numerical ratio, which can be expressed by means of two small integers. The names of the more important consonant intervals, with the respective ratios, are as follows:

$\begin{array}{llll}\text { Unison } & 1: 1 & \text { Octave } & 1: 2 \\ \text { Fifth } & 2: 3 & \text { Fourth } & 3: 4 \\ \text { Major Third } & 4: 5 & \text { Minor Sixth } & 5: 8 \\ \text { Minor Third } & 5: 6 & \text { Major Sixth } & 3: 5 .\end{array}$

The ear has of course no appreciation of the numerical relations themselves; but each interval is more or less sharply " defined," in the sense that a slight mistuning of either note is at once detected by the beats, and consequent sensation of roughness, which are produced. The explanation of these latter peculiarities must be deferred for the present. 
The names given to the various intervals are in a sense accidental, and refer to the relative positions of the notes on the ordinary "diatonic scale." This is based on the "major chord," which is a combination of three notes forming a Major and a Minor Third; i.e. their frequencies are as $4: 5: 6$. If we start from any arbitrary note, which we will call $\mathrm{C}$, as keynote, the two notes which lie a Fifth above and below it are called the "dominant" (G) and the "subdominant" (F) respectively. If we form the major chord from $\mathrm{C}$ we get the notes $\mathrm{E}=\frac{5}{4} \mathrm{C}$, and $G=\frac{3}{2} C$. Again if we form the major chord from $G$ we get the notes $\mathrm{B}=\frac{5}{4} \mathrm{G}=\frac{15}{8} \mathrm{C}$, and $d=\frac{3}{2} \mathrm{G}=\frac{9}{4} \mathrm{C}$. The latter falls outside the octave beginning with $\mathrm{C}$; the corresponding note within the octave is $\mathrm{D}=\frac{9}{8} \mathrm{C}$. Lastly, forming the major chord from $\mathrm{F}$, we get $\mathrm{A},=\frac{5}{4} \mathrm{~F}=\frac{5}{4} \times \frac{3}{2} \mathrm{C}=\frac{5}{6} \mathrm{C}$, the octave of which is $\mathrm{A}=\frac{5}{3} \mathrm{C}$, and $\mathrm{C}$ itself. We thus obtain the scale of seven notes whose frequencies are proportional to the numbers here given:

$\begin{array}{ccccccc}\text { C } & \text { D } & \text { E } & \text { F } & \text { G } & \text { A } & \text { B } \\ 1 & \frac{9}{8} & \frac{5}{4} & \frac{4}{3} & \frac{3}{2} & \frac{5}{3} & \frac{15}{8} \\ 24 & 27 & 30 & 32 & 36 & 40 & 45\end{array}$

This is continued upwards and downwards in octaves; the same letters are repeated as the names of the notes, but the various octaves may be distinguished by difference of type, and by accents or suffixes. The precise pitch of the key-note is so far arbitrary; it determines, and is determined by, that of any other note in the scale. Among musicians the standard has varied in different places and at different times, the general tendency being in the direction of a rise. German physical writers, including Helmholtz, have followed a standard which assigns to a certain A a frequency of 440 *. On this basis we have the following frequencies for a certain range of the scale:
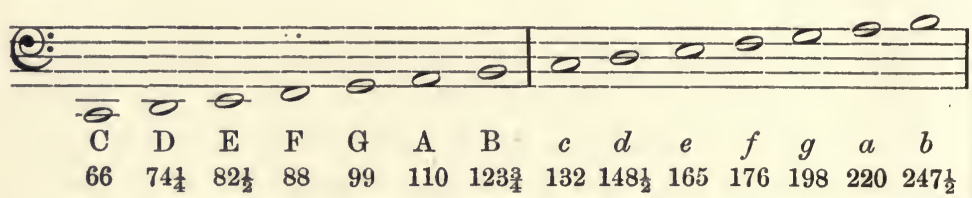

* This makes $c^{\prime}=264$. Physical instrument makers now often take $c^{\prime}=256$, which is convenient on account of its continued divisibility by 2 . 


\section{CHAPTER I}

\section{THEORY OF VIBRATIONS}

\section{The Pendulum.}

A vibrating body, such as a string or a bar or a plate, cannot give rise to a sound except in so far as it acts on the surrounding medium, which in turn exerts a certain reaction on the body. The reaction is however in many cases so slight that its effects only become sensible after a large number of oscillations. Hence, to simplify matters, we begin by ignoring it, and investigate the nature of the vibrations of a mechanical system considered as completely isolated.

The theory of vibrations begins, historically and naturally, with the pendulum. With this simple apparatus we are able to illustrate, in all essentials, many important principles of acoustics, the mere differences of scale as regards amplitude and period, enormous as they are, being unimportant from the dynamical point of view.

A particle of mass $M$, suspended from a fixed point $O$ by a light string of length $l$, is supposed to make small oscillations, in a vertical plane, about its position of equilibrium. If the inclination of the string to the vertical never exceeds a few degrees, the vertical displacement of the particle may (to a first approximation) be neglected, and the tension $(P)$ of the string may be

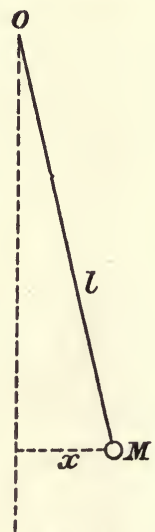

Fig. 1. equated to the gravity $M g$ of the particle. Since the horizontal displacement $(x)$ is affected only by the horizontal component of the tension, we have

$$
M \frac{d^{2} x}{d t^{2}}=-P \frac{x}{l}=-M g \frac{x}{l} .
$$


If we put

$$
n^{2}=g / l \text {, }
$$

this becomes

$$
\frac{d^{2} x}{d t^{2}}+n^{2} x=0 \text {; }
$$

and the solution is

$$
x=A \cos n t+B \sin n t,
$$

where the constants $A, B$ may have any values. That this formula really satisfies (3) is verified at once by differentiation; and since it contains two arbitrary constants $A, B$, we are able to adapt it to any prescribed initial conditions of displacement and velocity. Thus if, when $t=0$, we are to have $x=x_{0}$, $d x / d t=u_{0}$, we find

$$
x=x_{0} \cos n t+\frac{u_{0}}{n} \sin n t .
$$

It is of course necessary, in the application to the pendulum, that the initial conditions should be such as are consistent with the assumed "smallness" of the oscillations. Thus in (5) we must suppose that the ratios $x_{0} / l$ and $u_{0} / n l$ are both small. In virtue of (2) the latter ratio is equal to $\sqrt{ }\left(u_{0}^{2} / g l\right)$, so that $u_{0}$ must be small compared with the velocity "due to" half the length of the pendulum.

\section{Simple-Harmonic Motion.}

If in $\S 4(4)$ we put

$$
A=a \cos \epsilon, \quad B=-a \sin \epsilon,
$$

as is always possible by a suitable choice of $a$ and $\epsilon$, we get

$$
x=a \cos (n t+\epsilon) \text {. }
$$

The particular type of vibration represented by this formula is of fundamental importance. It is called a "simple-harmonic," or (sometimes) a "simple" vibration. Its character is best exhibited if we imagine a geometrical point $Q$ to describe a circle of radius $a$ with the constant angular velocity $n$. The orthogonal projection $P$ of $Q$ on a fixed diameter $A O A^{\prime}$ will move exactly according to

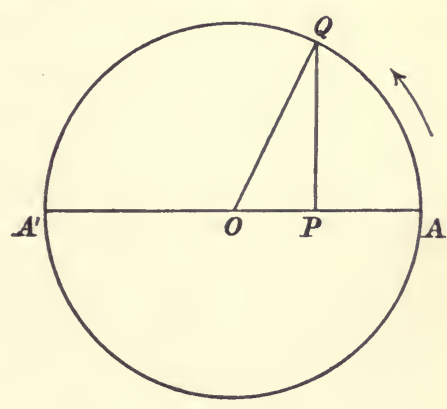

Fig. 2. 
the formula (2), provided it be started at the proper instant. The angle $n t+\epsilon(=A O Q)$ is called the "phase"; and the elements $a, \epsilon$ are called the "amplitude" and the "initial phase," respectively. The interval $2 \pi / n$ between two successive transits through the origin in the same direction is called the "period." In acoustics, where we have to deal with very rapid vibrations, it is usual to specify, instead of the period, its reciprocal the "frequency" $(N)$, i.e. the number of complete vibrations per second; thus

$$
N=n / 2 \pi * \text {. }
$$

In the case of the pendulum, where $n=\sqrt{ }(g / l)$, the period is $2 \pi \sqrt{ }(l / g)$. As in the case of all other dynamical systems which we shall have occasion to consider, this is independent of the amplitude so long as the latter is small $\uparrow$.

The velocity of $P$ in any position is

$$
\frac{d x}{d t}=-n a \sin (n t+\epsilon)=-n \cdot P Q,
$$

as appears also by resolving the velocity $(n a)$ of $Q$ parallel to $O A$.

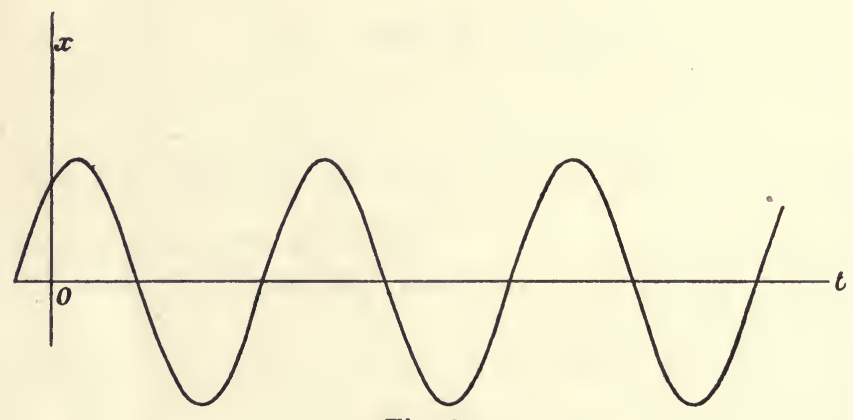

Fig. 3.

In all cases of rectilinear motion of a point the method of graphical representation by means of a curve constructed with

* The want of a separate name for the angular velocity $n$ in the auxiliary circle is sometimes felt. In the theory of the tides the term "speed" was introduced by Lord Kelvin. As an alternative term in acoustics the word "rapidity" may perhaps be suggested.

+ This observation was made by Galileo in 1583 , the pendulum being a lamp which hangs in the cathedral of Pisa. 
the time $t$ as abscissa and the displacement $x$ as ordinate is of great value. This is called the "curve of positions," or the "space-time curve." In experimental acoustics numerous mechanical and optical devices have been contrived by means of which such curves can be obtained. In the present case of a simple-harmonic vibration, the formula (2) shews that the curve in question is the well-known "curve of sines."

\section{Further Examples.}

The governing feature in the theory of the pendulum is that the force acting on the particle is always towards the position of equilibrium and (to a sufficient approximation) proportional to the displacement therefrom. All cases of this kind are covered by the differential equation

$$
M \frac{d^{2} x}{d t^{2}}=-K x,
$$

and the oscillation is therefore of the type (2) of $\S 5$, with $n^{2}=K / M$. The motion is therefore simple-harmonic, with the frequency

$$
N=\frac{n}{2 \pi}=\frac{1}{2 \pi} \sqrt{\left(\frac{K}{M}\right)}
$$

determined solely by the nature of the system, and independent of the amplitude. The structure of this formula should be noticed, on account of its wide analogies. The frequency varies as the square root of the ratio of two quantities, one of which $(K)$ measures the elasticity, or the degree of stability, of the system, whilst the other is a coefficient of inertia.

Consider, for example, the vertical oscillations of a mass $M$ hanging from a fixed support by a helical spring. In conformity with Hooke's law of elasticity, we assume that the force exerted by the spring is equal to the increase of length multiplied by a certain constant $K$, which may be called the "stiffness" of the particular spring. In the position of equilibrium the tension of the spring exactly balances the gravity $M g$; and if $M$ be displaced downwards through a space $x$, an additional force $K x$ towards this position

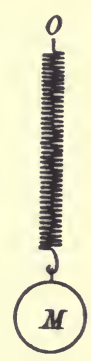

Fig. 4. is called into play, so that the equation of motion is of 
the type (1). The inertia of the spring itself is here neglected*.

Again, suppose we have a mass $M$ attached to a wire which is tightly stretched between two fixed points with a tension $P$. We neglect gravity and the inertia of the wire

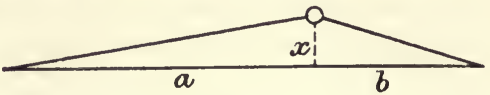

Fig. 5 . itself; and we further assume the lateral displacement $(x)$ to be so small that the change in tension is a negligible fraction of $P$. If $a, b$ denote the distances of the attached particle from the two ends, we have

$$
M \frac{d^{2} x}{d t^{2}}=-P \frac{x}{a}-P \frac{x}{b}
$$

which is of the same form as $\S 4(3)$, with $n^{2}=P(a+b) / M a b$. The frequency is therefore

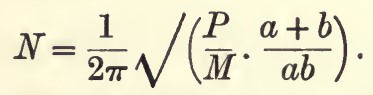

This case is of interest because acoustical frequencies can easily be realized. Thus if the tension be 10 kilogrammes, and a mass of 5 grammes be attached at the middle, the wire being $50 \mathrm{~cm}$. long, we find $N=63$.

\section{Dynamics of a System with One Degree of Freedom.} Free Oscillations.

The above examples are all concerned with the rectilinear motion of a particle, but exactly the same type of vibration is met with in every case of a dynamical system of one degree of freedom oscillating freely, through a small range, about a configuration of stable equilibrium.

A system is said to have "one degree of freedom" when the various configurations which it can assume can all be specified by assigning the proper values to a single variable element or "coordinate." Thus, the position of a cylinder (of any form of section) rolling on a horizontal plane is defined by the angle through which it has turned from some standard position. A system of two particles attached at different points of a string whose ends $A, B$ are fixed has one degree of freedom

\footnotetext{
* A correction on this account is investigated in $\S 7$.
} 
if it be restricted to displacements in the vertical plane through $A, B$, for the configuration may be specified by the inclination of any one of the strings to the horizontal. Again, the configuration of a steam-engine and of the whole train of machinery which it actuates is defined by the angular coordinate of the flywheel. The variety of such systems is endless, but if we exclude frictional or other dissipative forces the whole motion of the system when started anyhow and left to itself is

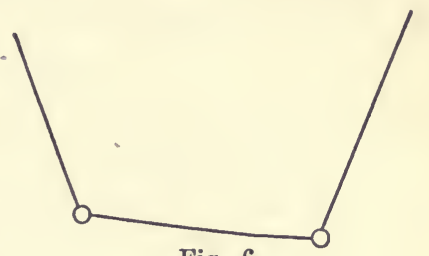

Fig. 6. governed by the equation of energy. And in the case of small oscillations about stable equilibrium, the differential equation of motion, as we shall see, reduces always to the type $\S 6(1)$.

We denote by $q$ the variable coordinate which specifies the configuration. As in the case of Fig. 6, this may be chosen in various ways, but the particular choice made is immaterial. From the definition of the system it is plain that each particle is restricted to a certain path. If in consequence of an infinitesimal variation $\delta q$ of the coordinate a particle $m$ describes an element $\delta s$ of its path, we have $\delta s=\alpha \delta q$, where $\alpha$ is a coefficient which is in general different for different particles, and also depends on the particular configuration $q$ from which the variation is made. Hence, dividing by the time-element $\delta t$, the velocity of this particle is $v=\alpha d q / d t$, or in the fluxional notation*, $v=\alpha \dot{q}$.

Hence the total kinetic energy, usually denoted by $T$, is

$$
\begin{array}{r}
T=\frac{1}{2} \Sigma\left(m v^{2}\right)=\frac{1}{2} a \dot{q}^{2}, \\
\quad a=\Sigma\left(m \alpha^{2}\right), \quad \ldots .
\end{array}
$$

where

the summation $\Sigma$ embracing all the particles of the system.

The coefficient $a$ is in general a function of $q$; it may be called the "coefficient of inertia" for the particular configuration $q$. For example, in the case of the rolling cylinder referred

* The use of dots to denote differentiations with respect to $t$ was revived by Lagrange in the Mécanique Analytique (1788), and again in later times by Thomson and Tait. We write $\dot{q}$ for $d q / d t$ and $\ddot{q}$ for $d^{2} q / d t^{2}$. 
to above, it is the (usually variable) moment of inertia about the line of contact with the horizontal plane, provided $q$ denote the angular coordinate.

The potential energy of the system, since it depends on the configuration, will be a function of $q$ only. If we denote it by $V$, the conservation of energy gives

$$
\frac{1}{2} a \dot{q}^{2}+V=\text { const. }
$$

provided the system be free from extraneous forces. The value of the constant is of course determined by the initial circumstances. If we differentiate (3) with respect to $t$, the resulting equation is divisible by $\dot{q}$, and we obtain

$$
a \ddot{q}+\frac{1}{2} \frac{d a}{d q} \dot{q}^{2}+\frac{d V}{d q}=0
$$

which may be regarded as the equation of free motion of the system, with the unknown reactions between its parts eliminated. In the application to small oscillations it greatly simplifies.

In order that there may be equilibrium the equation (4) must be satisfied by $q=$ const. This requires that $d V / d q=0$; i.e. an equilibrium configuration is characterised by the fact that the potential energy is "stationary" in value for small deviations from it. By adding or subtracting a constant, we can choose $q$ so as to vanish in the equilibrium configuration which is under consideration, whence, expanding in powers of the small quantity $q$, we have

$$
V=\text { const. }+\frac{1}{2} c q^{2}+\ldots,
$$

the first power of $q$ being absent on account of the stationary property. The constant $c$ is positive if the equilibrium configuration be stable, and $V$ accordingly then a minimum*. It may be called the "coefficient of stability."

If we substitute from (5) in (4), and omit terms of the second order in $q, \dot{q}$, we obtain

$$
a \ddot{q}+c q=0,
$$

where $a$ may now be supposed to be constant, and to have the value corresponding to the equilibrium configuration.

* In the opposite case the solution of (6) below would involve real exponen. tials instead of circular functions, indicating instability. 
Since (6) is of the same type as $§ 6(1)$, with

$$
n^{2}=c / a
$$

the variation of $q$ is simple-harmonic, say

$$
q=C \cos (n t+\epsilon)
$$

with the frequency

$$
N=\frac{1}{2 \pi} \sqrt{ }\left(\frac{c}{a}\right)
$$

Moreover, since the displacement of any particle of the system along its path, from its equilibrium position, is proportional to $q$ (being equal to $\alpha q$ in the above notation), we see that each particle will execute a simple-harmonic vibration of the above frequency, and that the different particles will keep step with one another, passing through their mean positions simultaneously. The amplitudes of the respective particles are moreover in fixed ratios to one another, the absolute amplitude, and the phase, being alone arbitrary, i.e. dependent on the particular initial conditions.

The kinetic and potential energies are respectively

$$
\left.\begin{array}{l}
T=\frac{1}{2} a \dot{q}^{2}=\frac{1}{2} n^{2} a C^{2} \sin ^{2}(n t+\epsilon), \\
V=\frac{1}{2} c q^{2}=\frac{1}{2} c C^{2} \cos ^{2}(n t+\epsilon),
\end{array}\right\}
$$

the sum being

$$
T+V=\frac{1}{2} n^{2} a C^{2}=\frac{1}{2} c C^{2},
$$

in virtue of (7). Since the mean values of $\sin ^{2}(n t+\epsilon)$ and $\cos ^{2}(n t+\epsilon)$ are obviously equal, and therefore each $=\frac{1}{2}$, the energy is on the average half kinetic and half potential.

The application of the theory to particular cases requires only the calculation of the coefficients $a$ and $c$, the latter being (in mechanical problems) usually the more troublesome. In the case of a body attached to a vertical wire, and making torsional oscillations about the axis of the wire, $a$ is the moment of inertia about this axis, and $c$ is the modulus of torsion, i.e. $c q$ is the torsional couple when the body is turned through an angle $q$.

Again in the case of a mass suspended by a coiled spring (Fig. 4), if we assume that the vertical displacement of any point of the spring is proportional to its depth $z$ below the 
point of suspension in the unstrained state, the kinetic energy is given by

$$
\begin{aligned}
2 T & =M \dot{q}^{2}+\int_{0}^{l}(z / l)^{2} \dot{q}^{2} \rho d z \\
& =\left(M+\frac{1}{3} \rho l\right) \dot{q}^{2}, \quad \ldots . .
\end{aligned}
$$

if $\rho$ be the line-density, $l$ the unstretched length, and $q$ the displacement of the weight. The inertia of the spring can therefore be allowed for by imagining the suspended mass to be increased by one-third that of the spring.

\section{Forced Oscillations of a Pendulum.}

The vibrations so far considered are "free," i.e. the system is supposed subject to no forces except those incidental to its constitution and its relation to the environment. We have now to examine the effect of disturbing forces, and in particular that of a force which is a simple-harmonic function of the time. This kind of case arises when one vibrating body acts on another under such conditions that the reaction on the first body may be neglected.

For definiteness we take the case of a mass movable in a straight line, the subsequent generalization $(\$ 9)$ being a very simple matter. The equation (1) of $\S 6$ is now replaced by

$$
M \frac{d^{2} x}{d t^{2}}=-K x+F \cos p t
$$

the last term representing the disturbing force, whose amplitude $F$, and frequency $p / 2 \pi$, are regarded as given*. If we write

$$
K / M=n^{2}, \quad F / M=f,
$$

we have

$$
\frac{d^{2} x}{d t^{2}}+n^{2} x=f \cos p t
$$

The complete solution of this equation is

$$
x=A \cos n t+B \sin n t+\frac{f}{n^{2}-p^{2}} \cos p t,
$$

as is easily verified by differentiation.

The first part of this, with its arbitrary constants $A, B$, represents a free vibration of the character explained in $\S 5$,

* The slightly more general case where the force is represented by $F \cos (p t+a)$ can be allowed for by changing the origin from which $t$ is reckoned. 
with the frequency $n / 2 \pi$ proper to the system. - On this is superposed a "forced vibration" represented by the last term. This is of simple-harmonic type, with the frequency $p / 2 \pi$ of the disturbing force, and the phase is the same as that of the force, or the opposite, according as $p \lesseqgtr n$, i.e. according as the imposed frequency is less or greater than the natural frequency.

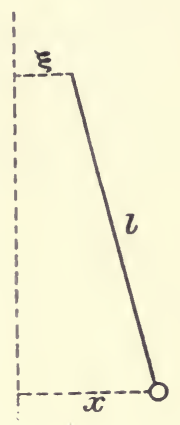

Fig. 7.

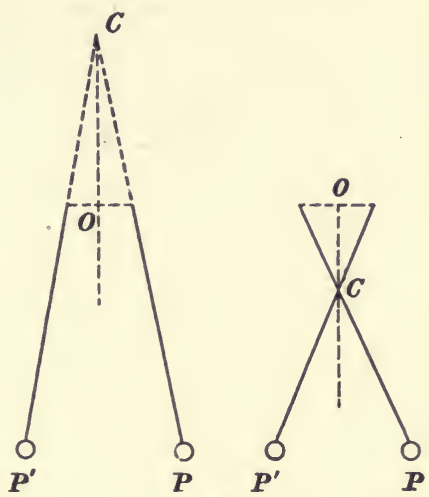

Fig. 8.

The above theory is easily illustrated by means of the pendulum. If the upper end of the string, instead of being fixed, is made to execute a horizontal motion in which the displacement at time $t$ is $\xi$ (Fig. 7), the equation of motion (1) of $\S 4$ is replaced by

or

$$
\begin{gathered}
M \frac{d^{2} x}{d t^{2}}=-M g \frac{x-\xi}{l}, \\
\frac{d^{2} x}{d t^{2}}+n^{2} x=n^{2} \xi .
\end{gathered}
$$

This is the same as if the upper end were fixed, and the bob were subject to a horizontal force whose accelerative effect is $n^{2} \xi$. If as a particular case we take

$$
\xi=\alpha \cos p t,
$$

we get the form (3), with $f=n^{2} \alpha$. The annexed Fig. 8 represents the forced oscillation in the two cases of $p<n$ and $p>n$, respectively. The pendulum oscillates as if $C$ were a fixed 
point, the distance $C P$ being equal to the length of the simple pendulum whose free period is equal to the imposed period $2 \pi / p$.

This example is due to Young*, who applied it to illustrate the dynamical theory of the tides, where the same question of phase arises. It appears from this theory that the tides in an imagined equatorial belt of ocean, of a breadth not exceeding a few degrees of latitude, and of any depth comparable with the actual depth of the sea, would be "inverted," i.e. there would be low water beneath the moon, and high water in longitudes $90^{\circ} \mathrm{E}$. and $\mathrm{W}$. from it, the reason being that the period of the disturbing force (viz. 12 lunar hours) is less than the corresponding free period, so that there is opposition of phase.

The arbitrary constants in the complete solution (4) are determined by the initial conditions. Suppose, for example, that the body starts from rest in the zero position at the instant $t=0$. We find

$$
x=\frac{f}{p^{2}-n^{2}}(\cos n t-\cos p t),
$$

as may be immediately verified.

When the imposed frequency $p / 2 \pi$ is nearly equal to the natural period, the last term in (4) becomes very large, and it may be that the assumption as to the smallness of $x$ on which the equation (1) is usually based (as in the case of the pendulum) is thereby violated. The result expressed by (4) is then not to be accepted without reserve, but we have at all events an indication of the reason why an amplitude of abnormal amount ensues whenever there is approximate agreement between the free and the forced period.

In the case $(p=n)$ of exact coincidence between the two periods, the solution (4) becomes altogether unmeaning, but an intelligible result may be obtained if we examine any particular.

* Dr Thomas Young (1773-1829), famous for his researches on light, and other branches of physics. The elementary theory of free and forced oscillations was given by him in an article on "A Theory of the Tides, including the consideration of Resistance," Nicholson's Journal, 1813 ; Miscellaneous Works, London, 1855, vol. II., p. 262. 
case in which the initial conditions are definite. Thus, in the case of (8), the formula may be written

$$
x=\frac{f}{p+n} \cdot \frac{\sin \frac{1}{2}(p-n) t}{\frac{1}{2}(p-n)} \cdot \sin \frac{1}{2}(p+n) t ;
$$

and as $p$ approaches equality with $n$ this tends to the limiting form.

$$
x=\frac{f}{2 n} t \sin n t .
$$

This may be described (roughly) as a simple vibration whose amplitude increases proportionally to $t$. For a reason just indicated this is only valid as a representation of the earlier stages of the motion.

The case of a disturbing force of more general character may be briefly noticed. The differential equation is then of the form

$$
\frac{d^{2} x}{d t^{2}}+n^{2} x=f(t)
$$

The method of solution, by variation of parameters, or otherwise, is explained in books on differential equations. The result, which may easily be verified, is

$$
x=\frac{1}{n} \sin n t \int f(t) \cos n t d t-\frac{1}{n} \cos n t \int f(t) \sin n t d t .
$$

It is unnecessary to add explicitly terms of the type $A \cos n t+B \sin n t$, which express the free vibrations, since these are already present in virtue of the arbitrary constants implied in the indefinite integrals.

If the force $f(t)$ is only sensible for a certain finite range of $t$, and if the particle be originally at rest in the position of equilibrium, we may write

$$
x=\frac{1}{n} \sin n t \int_{-\infty}^{t} f(t) \cos n t d t-\frac{1}{n} \cos n t \int_{-\infty}^{t} f(t) \sin n t d t,
$$

since this makes $x=0, d x / d t=0$ for $t=-\infty$. The vibration which remains after the force has ceased to be sensible is accordingly

where

$$
x=A \cos n t+B \sin n t,
$$

$$
A=-\frac{1}{n} \int_{-\infty}^{\infty} f(t) \sin n t d t, \quad B=\frac{1}{n} \int_{-\infty}^{\infty} f(t) \cos n t d t .
$$


For example, let

$$
f(t)=\frac{\mu}{\pi} \cdot \frac{\tau}{t^{2}+\tau^{2}}
$$

this represents a force which is sensible for a greater or less interval on both sides of the instant $t=0$, according to the value of $\tau$, the integral amount or impulse being $\mu^{*}$. By making $\tau$ sufficiently small we can approximate as closely as we please to the case of an instantaneous impulse. Since

$$
\int_{-\infty}^{\infty} \frac{\cos n t d t}{t^{2}+\tau^{2}}=\frac{\pi}{\tau} e^{-n \tau}, \int_{-\infty}^{\infty} \frac{\sin n t d t}{t^{2}+\tau^{2}}=0, \ldots . .(17) \dagger
$$

we have

$$
x=-\frac{\mu e^{-n \tau}}{n} \sin n t \text {. }
$$

The exponential factor shews the effect of spreading out the impulse. This effect is greater, the greater the frequency of the natural vibration.

\section{Forced Oscillations in any System with One Degree} of Freedom. Selective Resonance.

The generalization of these results offers no difficulty. When given extraneous forces act on a system with one degree of freedom, whose coordinate is $q$, the work which they perform in an infinitely small change of configuration, being proportional to $\delta q$, may be denoted by $Q \delta q$. The quantity $Q$ is called the "force" acting on the system, "referred to the coordinate $q . "$ For instance, if $q$ be the angular coordinate of a body which can rotate about a fixed axis, $Q$ is the moment of the extraneous forces about this axis.

It follows that in any actual motion of the system the rate at which extraneous forces are doing work is $Q \dot{q}$. The equation of energy now takes the form

$$
\frac{d}{d t}(T+V)=Q \dot{q}
$$

whence, inserting the value of $T$ from $\$ 7$ (1), we have

$$
a \ddot{q}+\frac{1}{2} \frac{d a}{d q} \dot{q}^{2}+\frac{d V}{d q}=Q .
$$

* The graph of this function is given, for another purpose, in Fig. 14, p. 33.

+ The former of these integrals is evaluated in most books on the Integral Calculus. 
When dealing with small motions in the neighbourhood of a configuration of equilibrium we may neglect terms of the second order as before. Hence, substituting the value of $V$ from $§ 7$ (5), we find

$$
a \ddot{q}+c q=Q .
$$

When $Q$ is of simple-harmonic type, varying (say) as $\cos p t$, the forced oscillation is given by

$$
q=\frac{Q}{c-p^{2} a}
$$

which is of course merely a generalized form of the last term in $\S 8$ (4).

Two special cases may be noticed. When $p$ is very small, (4) reduces to $q=Q / c$. This may be described as the "equilibrium" value* of the displacement, viz. it is the statical displacement which would be maintained by a constant force equal to the instantaneous value of $Q$. In other words, it is the displacement which would be produced if the system were devoid of inertia $(a=0)$. Denoting this equilibrium value by $\bar{q}$, we may write (4) in the form

$$
q=\frac{\bar{q}}{1-p^{2} / n^{2}},
$$

where, as in $\S 7, n$ denotes the speed of a free vibration.

When, on the other hand, $p$ is very great compared with $n$, (4) reduces to

$$
q=-Q / p^{2} a,
$$

approximately. This is almost the same as if the system were devoid of potential energy, the inertia alone having any sensible influence.

When two or more disturbing forces of simple-harmonic type act on a system, the forced vibrations due to them may be superposed by mere addition. Thus a disturbing force

$$
Q=f_{1} \cos \left(p_{1} t+\alpha_{1}\right)+f_{2} \cos \left(p_{2} t+\alpha_{2}\right)+\ldots
$$

will produce the forced oscillation

$$
q=\frac{f_{1}}{n^{2}-p_{1}{ }^{2}} \cos \left(p_{1} t+\alpha_{1}\right)+\frac{f_{2}}{n^{2}-p_{2}{ }^{2}} \cos \left(p_{2} t+\alpha_{2}\right)+\ldots
$$

* The name is taken from the theory of the tides, where the equilibrium tide-height is defined as that which would be maintained by the disturbing forces if these were to remain permanently at their instantaneous values. 
It will be observed that the amplitudes of the various terms are not proportional to those of the corresponding terms in the value of $Q$, owing to the difference in the denominators.

This is an illustration of a remark made in $\S 1$ that the simple-harmonic type is the only one which is unaltered in character when it is transmitted, the character of the composite vibration represented by (8) being different from that of the generating force. In particular if one of the imposed speeds $p_{1}, p_{2}, \ldots$ be nearly coincident with the natural speed $n$, the corresponding element in the forced vibration may greatly predominate over the rest. This is the theory of selective "resonance," so far as it is possible to develop it without reference to dissipative forces.

\section{Superposition of Simple Vibrations.}

The superposition of simple-harmonic motions in the same straight line has many important applications. For instance, the height of the tide at any station is the algebraic sum of a number of simple-harmonic components, the most considerable (at many stations) being those whose periods are half a lunar and half a solar day, respectively.

The composition of two simple vibrations may be illustrated by the geometrical method of Fig. 2. If two points $Q_{1}, Q_{2}$ describe concentric circles with the angular velocities $n_{1}, n_{2}$, their projections

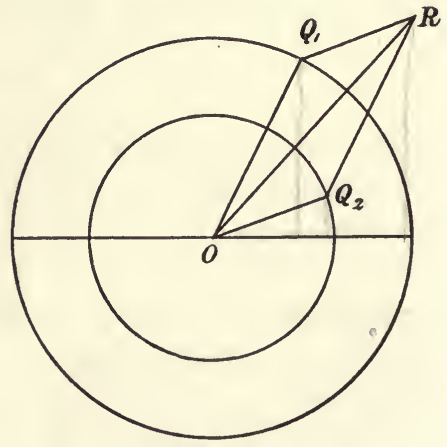

Fig. 9. on a fixed diameter will execute simple-harmonic vibrations of the forms

$$
x_{1}=a_{1} \cos \left(n_{1} t+\epsilon_{1}\right), \quad x_{2}=a_{2} \cos \left(n_{2} t+\epsilon_{2}\right),
$$

where $a_{1}, a_{2}$ are the radii of the two circles, and $\epsilon_{1}, \epsilon_{2}$ are the initial inclinations of the radii $O Q_{1}, O Q_{2}$ to the axis of $x$. The result of the superposition is

$$
x=x_{1}+x_{2},
$$


and it appears that the value of $x$ is the projection of $O R$, the diagonal of the parallelogram determined by $O Q_{1}, O Q_{2}$.

If $n_{1}=n_{2}$, the two component vibrations have the same period, the angle $Q_{1} O Q_{2}$ is constant, and the resultant vibration is simple-harmonic of the same period.

But if $n_{1}, n_{2}$ are unequal, the angle $Q_{1} O Q_{2}$ will vary between 0 and $180^{\circ}$, and $O R$ will oscillate between the values $a_{1} \pm a_{2}$. In Lord Kelvin's "tidal clock," the "hands" $O Q_{1}, O Q_{2}$ revolve in half a lunar and half a solar day, respectively, and the sides $Q_{1} R, Q_{2} R$ of the parallelogram are formed of rods jointed to these and to one another. The projection of $R$ then indicates the tide-height due to the superposition of the lunar and solar semidiurnal tides.

If the periods $2 \pi / n_{1}, 2 \pi / n_{2}$ are very nearly though not exactly equal, the angle $Q_{1} O Q_{2}$ will vary very little in the course of a single revolution of $O Q_{1}$ or $O Q_{2}$, and the resultant vibration may be described, in general terms, as a simple vibration whose amplitude fluctuates between the limits $a_{1} \pm a_{2}$. The period of a fluctuation is the interval in which one arm $O Q_{1}$ gains four right angles on the other, or $2 \pi /\left(n_{1}-n_{2}\right)$. Inverting, we see that the frequency of the fluctuations is the difference of the frequencies of the two constituent vibrations. We have here the reason for the alternation of "spring" and "neap" tides, according as the phases of the lunar and solar semidiurnal tides agree or are opposed. In acoustics we have the important phenomenon of " beats" between two tones of slightly different pitch. The contrast between the maximum and minimum amplitudes is of course greatest when the amplitudes $a_{1}, a_{2}$ of

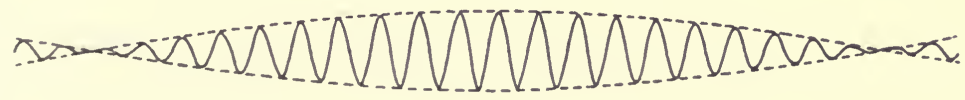

Fig. 10.

the primary vibrations are equal. We then have

$$
\begin{aligned}
x & =a_{1} \cos \left(n_{1} t+\epsilon_{1}\right)+a_{2} \cos \left(n_{2} t+\epsilon_{2}\right) \\
& =2 a \cos \left\{\frac{1}{2}\left(n_{1}-n_{2}\right) t+\frac{1}{2}\left(\epsilon_{1}-\epsilon_{2}\right)\right\} \cos \left\{\frac{1}{2}\left(n_{1}+n_{2}\right) t+\frac{1}{2}\left(\epsilon_{1}+\epsilon_{2}\right)\right\} .
\end{aligned}
$$

This may be described, in the same general manner as before, 
as a simple vibration whose period is $2 \pi / \frac{1}{2}\left(n_{1}+n_{2}\right)$, and whose amplitude oscillates between the limits 0 and $2 a$, in the time $\pi / \frac{1}{2}\left(n_{1}-n_{2}\right)$. This is illustrated graphically, with $x$ as ordinate and $t$ as abscissa, in Fig. 10, for the case of $n_{1}: n_{2}=41: 39$.

\section{Free Oscillations with Friction.}

The conception of a dynamical system as perfectly isolated and free from dissipative forces, which was adapted provisionally in $\$ 4-10$, is of course an ideal one. In practice the energy of free vibrations is gradually used up, or rather converted into other forms, although in most cases of acoustical interest the process is a comparatively slow one, in the sense that the fraction of the energy which is dissipated in the course of a single period is very minute.

To represent the effects of dissipation, whether this be due to causes internal to the system, or to the communication of energy to a surrounding medium, we introduce forces of resistance which are proportional to velocity. The forces in question are by hypothesis functions of the velocity*, and when the motion is small, the first power only need be regarded.

The equation of free motion of a particle about a position of equilibrium thus becomes

$$
M \frac{d^{2} x}{d t^{2}}=-K x-R \frac{d x}{d t},
$$

where $R$ is the coefficient of resistance. If we write

$$
\begin{aligned}
& K / M=n^{2}, \quad R / M=k, \\
& \frac{d^{2} x}{d t^{2}}+k \frac{d x}{d t}+n^{2} x=0 .
\end{aligned}
$$

The solution of this equation may be made to depend on that of $\$ 4$ (3) by the following artifice $\dagger$. We put

$$
x=y e^{-\frac{1}{2} k t} \text {, }
$$

* We shall see at a later stage (Chap. VIII) that the resistance of a medium may introduce additional forces depending on the acceleration. These have the effect of a slight apparent increase of inertia, and contribute nothing to the dissipation. It is unnecessary to take explicit account of them at present.

+ Another method of solution is given in $\S 20$. 
and obtain, on substitution,

$$
\frac{d^{2} y}{d t^{2}}+\left(n^{2}-\frac{1}{4} k^{2}\right) y=0 \text {. }
$$

We have now three cases to distinguish. If the friction be relatively small, more precisely if $k<2 n$, we may put

$$
n^{\prime 2}=n^{2}-\frac{1}{4} k^{2}
$$

and the solution of (3) is

$$
y=A \cos n^{\prime} t+B \sin n^{\prime} t,
$$

whence $\quad x=e^{-\frac{1}{2} k t}\left(A \cos n^{\prime} t+B \sin n^{\prime} t\right)$.

Changing the arbitrary constants, and putting

we have

$$
\begin{array}{r}
\tau=2 / k, \ldots \ldots . . \\
x=a e^{-t / \tau} \cos \left(n^{\prime} t+\epsilon\right) .
\end{array}
$$

This may be described as a modified simple-harmonic vibration in which the amplitude $\left(a e^{-t / \tau}\right)$ sinks asymptotically to 0 as $t$ increases. The time $\tau$ in which the amplitude is diminished in the ratio $1 / e$ is called the "modulus of decay." The relation between $x$ and $t$ is exhibited graphically in Fig. 11,

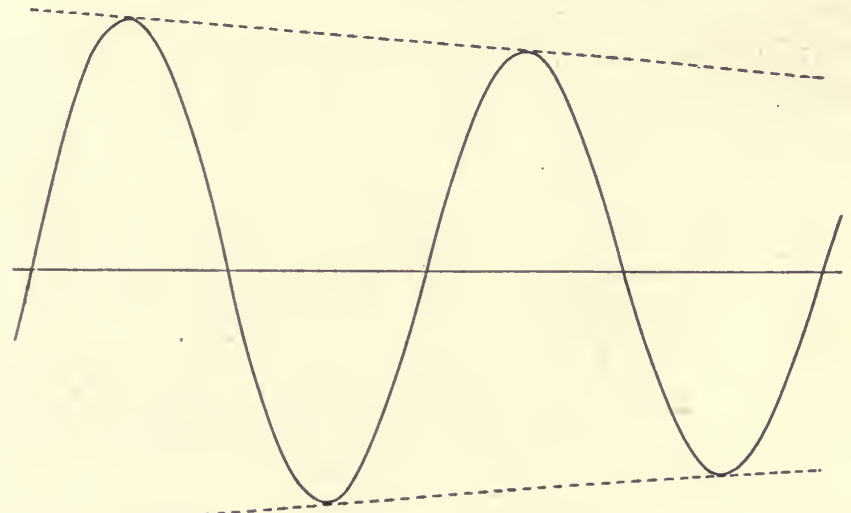

Fig. 11.

where the dotted lines represent portions of the exponential curves $x= \pm a e^{-t / \tau}$. For the sake of clearness the rapidity of decay is here taken to be much greater than it would be in any ordinary acoustical example. 
We have seen that a true simple-harmonic vibration may be regarded as the orthogonal projection of uniform motion in a circle. An analogous representation of the modified type (10) is obtained if we replace the circle by an equiangular spiral described with constant angular velocity $n^{\prime}$ about the pole 0 , in the direction in which the radius vector $r$ decreases*. The formula (10) is in fact equivalent to $x=r \cos \theta$, provided

$$
r=a e^{-t / r}, \theta=n^{\prime} t+\epsilon .
$$

Eliminating $t$ we have

$$
r=\alpha e^{-\beta \theta},
$$

where $\beta=\left(n^{\prime} \tau\right)^{-1}, \alpha=a e^{\beta \epsilon}$. This is the polar equation of the spiral in question. The curve in Fig. 12 corresponds in scale with Fig. 11.

In most acoustical applications the fraction $k / 2 n$, or $1 / n \tau$, is a very small quantity. In this case, the difference between $n$ and $n^{\prime}$ is a small quantity of the second order, and may usually be ignored; in other words, the effect of friction on the period is insensible. It may be noted that thequantity $1 / n \tau$, whose square is neglected, is the ratio of the period $2 \pi / n$ to the time $2 \pi \tau$

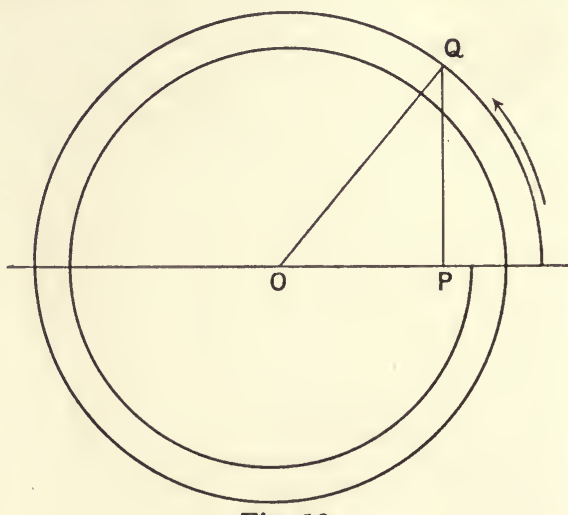

Fig. 12. in which the amplitude is diminished in the ratio $e^{-2 \pi}$ or $\frac{1}{535}$.

If $k$ be greater than $2 n$ the form of the solution of (3) is altered, viz. we have

whence

$$
y=A e^{\sqrt{ }\left(\frac{1}{4} k^{2}-n^{2}\right) t}+B e^{-\sqrt{ }\left(\frac{1}{4} k^{2}-n^{2}\right) t},
$$

$x=A e^{-\alpha t}+B e^{-\beta t}$,

if

$$
\left.\begin{array}{l}
\alpha \\
\beta
\end{array}\right\}=\frac{1}{2} k \mp \sqrt{ }\left(\frac{1}{4} k^{2}-n^{2}\right) \text {. }
$$

* This theorem was given in 1867 by P. G. Tait (1831-1901), Professor of Natural Philosophy at Edinburgh (1860-1901). 
The particle comes asymptotically to rest but does not oscillate; in fact we may easily see that it passes once at most through its zero position. This type of motion is realized in the case of a pendulum swinging in a very viscous liquid, and in "dead-beat" galvanometers and other electrical instruments, but it is 'of little interest in acoustics.

If $k=2 n$, exactly, the solution of (3) is of the form

$$
x=(A+B t) e^{-n t},
$$

as to which similar remarks may be made.

12. General Dissipative System with One Degree of Freedom. Effect of Periodic Disturbing Forces.

The effect of dissipation on the free motion of any system having one degree of freedom is allowed for by the assumption that there is a loss of mechanical energy at a rate proportional to the square of the generalized velocity, so that in the notation of $\S 7$

$$
\frac{d}{d t}\left(\frac{1}{2} a \dot{q}^{2}+\frac{1}{2} c q^{2}\right)=-b \dot{q}^{2}
$$

whence

$$
a \ddot{q}+b \dot{q}+c q=0 \text {. }
$$

This is of course the same as if we had introduced a frictional force $Q=-b \dot{q}$ in $\S 9(3)$.

The equation (1) has the same form as $§ 11$ (3), and the results will correspond if we put

$$
n^{2}=c / a, \quad \tau=2 a / b .
$$

When the dissipation is small, the rate of decay of the amplitude can be estimated by an independent method, due to Stokes*, which we shall often find useful. The period being practically unaffected by vicosity, a considerable number of oscillations can be fairly represented by

$$
q=C \cos (n t+\epsilon)
$$

provided $C$ and $\epsilon$ be gradually changed so as to fit the altering circumstances. The average energy over such an interval will be $\frac{1}{2} n^{2} a C^{2}$, approximately, by $\S 7$ (11); and the rate of dissipation will be

$$
b \dot{q}^{2}=\frac{1}{2} n^{2} b C^{2}\{1-\cos 2(n t+\epsilon)\},
$$

* Sir George Gabriel Stokes (1819-1903), Lucasian Professor of Mathematics at Cambridge (1849-1903). 
the mean value of which is $\frac{1}{2} n^{2} b C^{2}$. Equating the mean rate of decay of the energy to the mean dissipation, we get

$$
\frac{d}{d t}\left(\frac{1}{2} n^{2} a C^{2}\right)=-\frac{1}{2} n^{2} b C^{2},
$$

whence

$$
\frac{d C}{d t}+\frac{1}{2} \frac{b}{a} C=0
$$

or

$$
C=C_{0} e^{-t / \tau} \text {, }
$$

if $\tau=2 a / b$, as in (3).

When there are given extraneous forces in addition to the dissipative influences, the equation of energy takes the form

$$
\frac{d}{d t}\left(\frac{1}{2} a \dot{q}^{2}+\frac{1}{2} c q^{2}\right)=-\frac{1}{2} b \dot{q}^{2}+Q \dot{q}
$$

whence

$$
a \ddot{q}+b \dot{q}+c q=Q \text {. }
$$

As in $\$ 9$ we consider specially the case of a disturbing force of simple-harmonic type, say

$$
Q=C \cos p t \text {. }
$$

A particular solution of (9) is then obtained in the form

$$
q=F \cos p t+G \sin p t,
$$

provided the constants $F, G$ are properly chosen. The necessary conditions are found on substitution to be

If we put

$$
\left.\begin{array}{r}
\left(c-p^{2} a\right) F+p b G=C, \\
-p b F+\left(c-p^{2} a\right) G=0 .
\end{array}\right\}
$$

$$
c-p^{2} a=R \cos \alpha, \quad p b=R \sin \alpha,
$$

we find

$$
F=\frac{C}{R} \cos \alpha, \quad G=\frac{C}{R} \sin \alpha,
$$

whence*

$$
q=\frac{C}{R} \cos (p t-\alpha) \text {. }
$$

The values of $R$ and $\alpha$ are determined by

$$
R^{2}=\left(c-p^{2} a\right)^{2}+p^{2} b^{2}, \quad \tan \alpha=\frac{p b}{c-p^{2} a} .
$$

$R$ is to be taken positively, and $\alpha$ may be assumed to lie between 0 and $\pi$.

* A more rapid way of obtaining this solution is explained in $\S 20$. 
The equation (9) is still satisfied if we add to (15) terms representing a free oscillation; and these added terms are necessary in order to constitute a complete solution capable of adjustment to arbitrary initial conditions. The free vibration dies out, however, asymptotically, so that after the lapse of a sufficient time the forced vibration (15) is alone sensible.

The circumstances which affect the amplitude and phase of this forced vibration require careful attention. The amplitude is a maximum when $R^{2}$ is least, i.e. when

$$
p=n\left(1-\frac{b^{2}}{2 a c}\right)^{\frac{1}{2}},
$$

and the maximum amplitude is accordingly

$$
\frac{C}{n b}\left(1-\frac{b^{2}}{4 a c}\right)^{-\frac{1}{2}} \text {. }
$$

In most cases of interest $b^{2} / a c$ is a small quantity of the second order; the maximum is then $C / n b$, and occurs when $p=n$, very approximately.

Again, it appears from (15) and (16) that the phase of $q$ lags behind that of the disturbing force by an angle $\alpha$, which lies between 0 and $\frac{1}{2} \pi$, or between $\frac{1}{2} \pi$ and $\pi$, according as $p^{2}$ is less or greater than $c / a$, i.e. according as the imposed frequency is less or greater than the natural frequency. If, keeping $p$ constant, we diminish the dissipation-coefficient $b$, $\alpha$ tends to the limit 0 or $\pi$, respectively, in accordance with $\S 8$, where we found exact agreement or opposition of phase in the absence of resistance. But if, keeping $b$ constant, we make $p$ approach the value $n(=\sqrt{ }(c / a))$ which determines the frequency in the absence of dissipation, $\alpha$ tends to the limit $\frac{1}{2} \pi$, and the phases of $q$ and $Q$ differ by an amount corresponding to a quarter-period. This means that the maxima of the disturbing force are now synchronous with the maxima of the velocity $\dot{q}$.

Some light is thrown on these relations if we examine the case of a pendulum whose bob receives equal positive and negative instantaneous impulses alternately at regular intervals. It is seen at once from Fig. 13 that an impulse in the direction of motion accelerates or retards the phase of an otherwise free vibration, according as it precedes or follows 
(within the limits of a quarter-period) the instant of maximum velocity. Thus if when the particle is at $P$, on its way to 0 , the velocity be increased in the ratio of $P Q$ to $P Q_{1}$, the phase is accelerated by the angle $Q O Q_{1}$, whilst a similar impulse at $P^{\prime}$ would retard the phase by the angle $Q^{\prime} O Q_{1}^{\prime}$.

In order that no effect may be produced on the phase it is necessary that the impulse be delivered at the instant of passing through 0 . If we imagine that a small assisting impulse is given at every such passage, as in the case of the ordinary clock escapement, we have an illustration of the circumstances of maximum resonance. The period of the disturbing force is exactly equal to the natural period, and the force synchronizes with the velocity. The amplitude is determined by the consideration that the work done by the impulses must balance that lost by friction. The result is not essentially different if the impulse be dif-

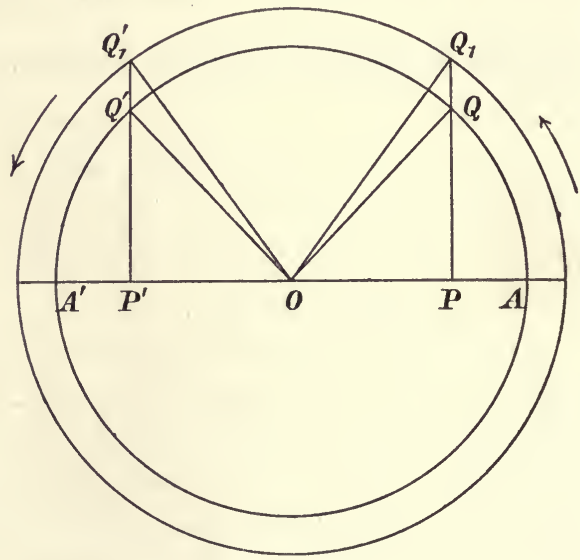

Fig. 13. fused symmetrically about 0 , as in the case of a simpleharmonic force, since the acceleration of phase on one side of $O$ is cancelled by the retardation on the other.

Next suppose that the assisting impulses are given each time the bob passes the symmetrically situated points $P, P^{\prime}$ inwards. There is an acceleration of phase at each impulse, and the period is shortened. This illustrates the case of a disturbing force whose period is less than the natural period, and whose maxima and minima precede the maxima and minima of the velocity. If on the other hand the impulses are given as the bob passes the points $P$ and $P^{\prime}$ outwards, there is a repeated retardation of phase, and the period is lengthened. This corresponds to the case of a disturbing force whose period 
is greater than the natural period; the maxima and minima of the force now follow those of the velocity. The reader is recommended to follow out in detail the argument here sketched, and to examine the effect of substituting a continuous simpleharmonic force for the series of disconnected impulses. An explanation may also be found, on the same principles, of the fact that a small frictional force varying as the velocity has no sensible effect on the free period.

We return to the analytical discussion. A difference of phase between the force and the displacement is essential in order that the disturbing force may supply energy to compensate that which is continually being lost by dissipation. When, as in $\S 9$, there is complete agreement (or opposition) of phase between $q$ and $Q$, the force is, in astronomical phrase, "in quadrature " with the velocity $\dot{q}$, that is, the phases differ by $\frac{1}{2} \pi$, and the total work done in a complete period is zero. Under the present circumstances the disturbing force is at any instant doing work at the rate

$$
\begin{aligned}
Q \dot{q} & =-\frac{p C^{2}}{R} \sin (p t-\alpha) \cos p t \\
& =\frac{p C^{2}}{2 R}\{\sin \alpha-\sin (2 p t-\alpha)\},
\end{aligned}
$$

the mean value of which is

$$
\frac{p C^{2}}{2 R} \sin \alpha=\frac{C^{2}}{2 b} \sin ^{2} \alpha .
$$

The same expression is of course obtained as the mean value of $b \dot{q}^{2}$, since the energy supplied by the disturbing force must exactly compensate, on the average, that which is continually being lost by dissipation, the mean energy stored in the system being constant.

It follows from (16) and (20) that the dissipation is greatest when $\alpha=\frac{1}{2} \pi$, or $p=n$, i.e. when the imposed frequency coincides with that of the free vibration in the absence of resistance. The maximum value is $\frac{1}{2} C^{2} / b$, being greater, of course, the smaller the value of $b$. 


\section{Effect of Damping on Resonance.}

The abnormal amplitude and dissipation which ensue whenever the imposed period is equal, or nearly equal, to the natural period constitute the phenomenon of "resonance," already referred to in $\S 8$, of which we shall have many acoustical examples in the sequel. It may be illustrated mechanically by giving a slight to-and-fro motion of suitable period to the point of suspension of a simple pendulum, or better by means of a double pendulum $(\$ 14)$, i.e. an arrangement in which two weights are attached at different points to a string hanging vertically from a fixed point. If the upper weight $(M)$ be considerable, whilst the lower one $(m)$ is relatively small, $M$ will swing almost exactly like the bob of a simple pendulum, the reaction of $m$ being slight. Under these conditions the motion of $m$ is practically that of a pendulum whose point of suspension has an imposed simple-harmonic vibration $(\$ 8)$, and if the length of the lower portion of the string be properly adjusted, a violent motion of $m$ may ensue.

One very important point remains to be mentioned. As the interval $p / n$ between the forced and the natural frequencies diverges from unity (on either side), the dissipation falls off from its maximum the more rapidly, the smaller the value of the frictional coefficient $b$. In other words, the greater the intensity of the resonance in the case of exact coincidence of frequencies, the narrower the range over which it is approximately equal to the maximum. For example, a tuning fork, even when mounted on a "resonance box," requires very perfect tuning in order that it may be excited perceptibly by the vibrations of another fork in the neighbourhood, whereas the column of air in a nearly closed vessel (e.g. a bottle or an organ pipe) will respond vigorously to a much wider range of frequencies. To elucidate the point, we notice that the expression $(20)$ of $\$ 12$ for the dissipation may be written

$$
\frac{C^{2}}{2 b} \sin ^{2} \alpha=\frac{1}{2} C^{2} \cdot \frac{p^{2} b}{\left(c-p^{2} a\right)^{2}+p^{2} b^{2}}=\frac{C^{2}}{4 \sqrt{ }(a c)} \cdot \frac{\beta}{\beta^{2}+\frac{1}{4}\left(\frac{p}{n}-\frac{n}{p}\right)^{2}},
$$

where

$$
\beta=\frac{1}{2} n b / c=1 / n \tau,
$$


in the notation of $\S 12(3)$. The second factor has its maximum value $1 / \beta$ when $p=n$, and evidently diminishes more rapidly, as $p / n$ deviates from unity, the smaller the value of $\beta$. The question may be conveniently illustrated graphically by constructing a curve which shall shew the dissipation corresponding to different frequencies. As regards the abscissa, it would in strictness be most proper to take, not the ratio $p / n$, but its logarithm, since equal intervals (in the musical sense) then correspond to equal lengths of the axis of $x$. We might therefore write

$$
x=\log \frac{p}{n}, y=\frac{\beta}{\beta^{2}+\sinh ^{2} x} ;
$$

but when, as usually happens, the sensible resonance is confined to a small range of $p / n$, we may use the simpler formulae

$$
x=\frac{p}{n}-1, y=\frac{\beta}{\beta^{2}+x^{2}} .
$$

The curve represented by the latter equation is symmetrical about the axis of $y$, and approaches the axis of $x$ asymptotically as $x$ increases. It is evident that if $\beta$ be increased in any

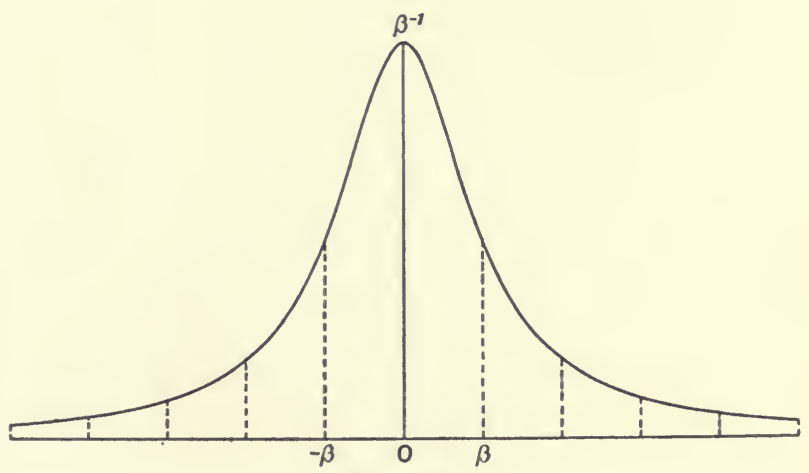

Fig. 14.

ratio, the new curve is obtained by increasing all the abscissae in that ratio, and diminishing the ordinates in the inverse ratio, the area $(\pi)$ included between the curve and the axis of $x$ being 
unaltered. The intensity sinks to one-half its maximum when $x^{2}=\beta^{2}$, or

$$
\frac{p}{n}=1 \pm \frac{1}{n \tau}
$$

Thus if the damping be such that a free vibration would have its amplitude diminished in the ratio $1 / e$ in $10,100,1000$ periods*, respectively, the corresponding values of the interval $p / n$ at which the dissipation would be reduced to one-half the maximum would be $1 \pm \cdot 016,1 \pm \cdot 0016,1 \pm \cdot 00016$. The curve in (4) is shewn in Fig. 14.

The above argument deals with the dissipation, which is the most important feature. The consideration of the square of the amplitude, or of the energy stored in the system, leads to very similar results, especially when the damping is slight.

\section{Systems of Multiple Freedom. Examples. The} Double Pendulum.

We approach the consideration of systems having any finite number of degrees of freedom. A system is said to have $m$ such degrees when $m$ independent variables, or "coordinates," are required and are sufficient to specify the various configurations which it can assume. The notion, first brought into formal prominence by Lord Kelvin $\dagger$, has a wide application in mechanism and in theoretical mechanics. In the case of the telescope of an altazimuth instrument or of an equatorial we have $m=2$; in the gyroscope, or (more generally) in any case of a rigid body free to turn about a fixed point, $m=3$; for a rigid structure or frame movable in two dimensions $m=3$; for a rigid structure freely movable in space $m=6$. The choice of the coordinates in any particular case can be made in an endless variety of ways, but the number is always determinate. Thus in technical mechanics we have the proposition that a rigid frame movable in one plane can be fixed by

* In an experiment by Lord Rayleigh, the number of periods for a particular tuning fork of $256 \mathrm{v} . \mathrm{s}$. was about 5900 . When a resonator was used the number fell to 3300. Theory of Sound, vol. Ir., p. 436.

† William Thomson, afterwards Lord Kelvin (1824-1907), Professor of Natural Philosophy at Glasgow 1846-99. The matter is explained in Thomson and Tait's Natural Philosophy, 2nd ed., \$§ 195-201 (1879). 
means of three links connecting any three points of it to any three fixed points in the plane*. Similarly any rigid threedimensional structure can be anchored firmly by six links connecting six points of it with six points fixed relatively to the earth.

Proceeding to the vibrations of a multiple system about a configuration of equilibrium, we begin as before with the examination of a few particular cases.

Take first the oscillations of a particle in a smooth bowl of any continuous shape. By means of suitable constraints, the particle may be restricted to oscillate in any given vertical plane through the lowest point 0 , e.g. by confining it between two frictionless guides infinitely close to one another. In general there will be a lateral pressure on one or other of these guides, which will however vanish if the plane in question passes through either of the principal directions of curvature at 0 . Hence two modes of free simple-harmonic vibration, in perpendicular directions, are possible, with speeds

$$
n_{1}=\sqrt{ }\left(g / R_{1}\right), \quad n_{2}=\sqrt{ }\left(g / R_{2}\right),
$$

where $R_{1}, R_{2}$, are the radii of curvature of the principal sections at 0 . On account of the assumed smallness of the motion, these vibrations may be superposed. The result is, if $x, y$ be horizontal rectangular coordinates through $O$,

$$
\left.\begin{array}{l}
x=A_{1} \cos n_{1} t+A_{2} \sin n_{1} t, \\
y=B_{1} \cos n_{2} t+B_{2} \sin n_{2} t .
\end{array}\right\}
$$

Since this contains four arbitrary constants, we can adjust the solution to given initial values of $x, y, \dot{x}, \dot{y}$.

This case is very neatly illustrated by Blackburn's pendulum + (Fig. 15). A weight hangs by a string $C P$ from a point $C$ of a string $A C B$ whose ends $A, B$ are fixed. The strings being supposed destitute of inertia, the point $P$ will always be in the same plane with $A, B, C$. Under this condition the locus of $P$ is the ring-shaped surface generated by revolving a circle

* Provided the directions of the three links be not concurrent (or parallel). There is a proviso of a more complex character in the case which follows; but such details need not occupy us here.

+ H. Blackburn, Professor of Mathematics at Glasgow 1849-79. 
with centre $C$ and radius $C P$, in the plane $A C B$, about $A B$ as an axis; and the principal radii of curvature at the lowest point $O$ are $R_{1}=C O, R_{2}=E O$, where $E$ is the point of $A B$ vertically above 0 . The corresponding directions of vibration are respectively in. and perpendicular to the plane $A B O$.

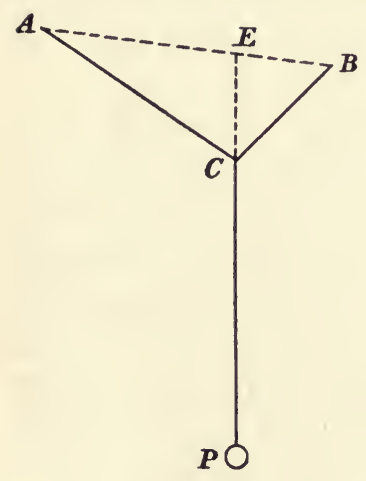

Fig. 15.

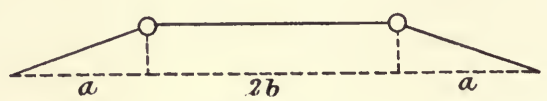

Fig. 16.

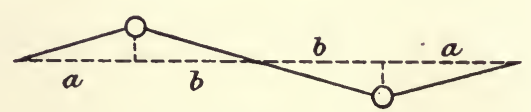

Fig. 17.

Another very simple case is that of two equal particles $M$ attached symmetrically at distances $a$ from the ends of a tense string, whose total length is, say, $2(a+b)$, so that $2 b$ denotes the length of the central portion. One obvious mode of simple-harmonic vibration is that in which the deflections of the two particles are always equal and of the same sign (Fig. 16). If $P$ be the tension of the string, the equation of motion of either particle is then

$$
M \frac{d^{2} x}{d t^{2}}=-P \frac{x}{a}
$$

and the speed is therefore

$$
n_{1}=\sqrt{ }(P / M a)
$$

In another mode the two deflections are equal in magnitude and opposite in sign, so that the middle point of the string is stationary (Fig. 17). The circumstances are therefore exactly the same as in $\S 6$, and the speed is

$$
n_{2}=\sqrt{ }\left(\frac{P}{M} \cdot \frac{a+b}{a b}\right),
$$

greater, as we should expect, than $n_{1}$. If we denote the 
deflections of the two particles by $x, y$, the superposition of the two modes gives

$$
\left.\begin{array}{l}
x=A \cos \left(n_{1} t+\epsilon_{1}\right)+B \cos \left(n_{2} t+\epsilon_{2}\right), \\
y=A \cos \left(n_{1} t+\epsilon_{1}\right)-B \cos \left(n_{2} t+\epsilon_{2}\right),
\end{array}\right\}
$$

where the four constants $A, B, \epsilon_{1}, \epsilon_{2}$ are arbitrary.

In the case of three attached particles the nature of the various modes is not so immediately obvious, even in the case of symmetry. We will suppose that the masses are equal, and that they divide the line into four equal segments $a$. Denoting the deflections by $x, y, z$, we have

$$
\left.\begin{array}{l}
M \frac{d^{2} x}{d t^{2}}=-P \frac{x}{a}-P \frac{x-y}{a}, \\
M \frac{d^{2} y}{d t^{2}}=-P \frac{y-x}{a}-P \frac{y-z}{a}, \\
M \frac{d^{2} z}{d t^{2}}=-P \frac{z}{a}-P \frac{z-y}{a} .
\end{array}\right\}
$$

If we put, for shortness, $\mu=P / M a$, these may be written

$$
\left.\begin{array}{r}
\frac{d^{2} x}{d t^{2}}+2 \mu x-\mu y=0, \\
-\mu x+\frac{d^{2} y}{d t^{2}}+2 \mu y-\mu z=0, \\
-\mu y+\frac{d^{2} z}{d t^{2}}+2 \mu z=0 .
\end{array}\right\}
$$

To ascertain the existence of modes of vibration in which the motion of each particle is simple-harmonic, with the same period and phase, we assume, tentatively,

$$
x=A \cos (n t+\epsilon), \quad y=B \cos (n t+\epsilon), \quad z=C \cos (n t+\epsilon) .
$$

It appears, on substitution in (8), that the equations will be satisfied provided

$$
\left.\begin{array}{r}
\left(n^{2}-2 \mu\right) A+\mu B=0 \\
\mu A+\left(n^{2}-2 \mu\right) B+\mu C=0, \\
\mu B+\left(n^{2}-2 \mu\right) C=0 .
\end{array}\right\}
$$

These three equations determine the two ratios $A: B: C$ and the value of $n^{2}$. Eliminating the former ratios we have

$$
\left(n^{2}-2 \mu\right)\left(n^{4}-4 \mu n^{2}+2 \mu^{2}\right)=0 \text {. }
$$


This is a cubic in $n^{2}$. One root is $n_{1}{ }^{2}=2 \mu$, and we find on reference to (10) that this makes $B_{1}=0, A_{1}=-C_{1}$, and therefore

$$
x=A_{1} \cos \left(n_{1} t+\epsilon_{1}\right), \quad y=0, \quad z=-A_{1} \cos \left(n_{1} t+\epsilon_{1}\right) .
$$

This mode might have been foreseen, and its frequency determined at once, as in the preceding example. The remaining roots of (11) are

$$
n_{3}^{2}=(2+\sqrt{ } 2) \mu, \quad n_{3}^{2}=(2-\sqrt{ } 2) \mu ;
$$

and it appears from (10) that these make

$$
A_{2}=C_{2}, \quad B_{2}=-\sqrt{ } 2 A_{2} \text {, and } A_{3}=C_{3}, \quad B_{3}=\sqrt{ } 2 A_{3} \text {, }
$$

respectively. The corresponding modes are therefore

$$
\begin{array}{r}
x=A_{2} \cos \left(n_{2} t+\epsilon_{2}\right), \quad y=-\sqrt{ } 2 A_{2} \cos \left(n_{2} t+\epsilon_{2}\right), \\
z=A_{2} \cos \left(n_{2} t+\epsilon_{2}\right),
\end{array}
$$

and

$$
\begin{array}{r}
x=A_{3} \cos \left(n_{3} t+\epsilon_{3}\right), \quad y=\sqrt{ } 2 A_{3} \cos \left(n_{3} t+\epsilon_{3}\right), \\
z=A_{3} \cos \left(n_{3} t+\epsilon_{3}\right) .
\end{array}
$$

These are shewn, along with the former mode, in Fig. 18. The complete solution of the equations is obtained by superposition of (12), (13) and (14), and contains the six arbitrary constants $A_{1}, A_{2}, A_{3}, \epsilon_{1}, \epsilon_{2}, \epsilon_{3}$.

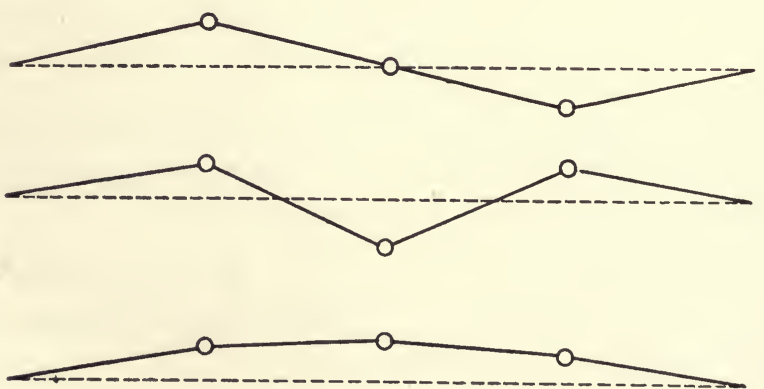

Fig. 18.

We conclude these illustrations with the case of the double pendulum, where we are entirely dependent on general method. A mass $M$ hangs from a fixed point by a string of length $a$, and a second mass $m$ hangs from $M$ by a string of length $b$. For simplicity we suppose the motion confined to one vertical 
plane. The horizontal excursions $x, y$ of $M, m$ respectively being supposed small, the tensions of the upper and lower strings will be $(M+m) g$ and $m g$, approximately. The equations of motion are therefore

$$
\left.\begin{array}{l}
M \frac{d^{2} x}{d t^{2}}=-(M+m) g \frac{x}{a}+m g \frac{y-x}{b}, \\
m \frac{d^{2} y}{d t^{2}}=-m g \frac{y-x}{b} .
\end{array}\right\}
$$

To find the possible modes of simple-harmonic vibration we assume

$$
x=A \cos (n t+\epsilon), \quad y=B \cos (n t+\epsilon) .
$$

The equations are satisfied provided

$$
\left.\begin{array}{r}
\left\{(1-\mu) n^{2}-g\left(\frac{1}{a}+\frac{\mu}{b}\right)\right\} A+\mu \frac{g}{b} B=0, \\
\frac{g}{b} A+\left(n^{2}-\frac{g}{b}\right) B=0,
\end{array}\right\}
$$

where

$$
\mu=m /(M+m) \text {. }
$$

Eliminating the ratio $A: B$, we find

$$
(1-\mu) n^{4}-g\left(\frac{1}{a}+\frac{1}{b}\right) n^{2}+\frac{g^{2}}{a b}=0,
$$

which is a quadratic in $n^{2}$. The condition for real roots, viz.

$$
g^{2}\left(\frac{1}{a}-\frac{1}{b}\right)^{2}+4 \mu \frac{g^{2}}{a b}>0 \text {, }
$$

is obviously always fulfilled. It is further easily seen that both roots are positive, so that $n$ also is real.

The problem includes a number of interesting special cases, but we will only notice one or two. If the ratio $\mu,=m /(M+m)$, be small, the two roots of (19) are $n_{1}^{2}=g / a$, $n_{2}{ }^{2}=g / b$, approximately. In the former case $M$ oscillates like the bob of a simple pendulum of length $a$, whilst $m$ executes what may be regarded as a forced oscillation

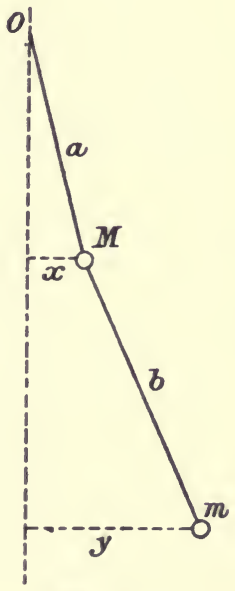

Fig. 19 
of the corresponding frequency; this case has already been referred to in $\S 13$. In the second mode the ratio $A: B$ is small, as appears from the second of equations (17); $M$ is then nearly at rest, whilst $m$ oscillates like the bob of a pendulum of length $b$.

Since the expression on the left-hand side of (20) cannot vanish, the two frequencies can never exactly coincide, but they become approximately equal if $a=b$, nearly, and $\mu$ is small. A curious phenomenon may then present itself. The motion of each mass, being made up of two superposed simple-harmonic vibrations of nearly equal period, may fluctuate greatly in extent, and if the amplitudes of the two vibrations are equal we have periods of approximate rest, as explained in $\S 10$. The motion then appears to be transferred alternately from $m$ to $M$, and from $M$ to $m$, at regular intervals*.

If, on the other hand, $M$ is small compared with $m, \mu$ is nearly equal to unity, and the two roots of (19) are $n^{2}=g /(a+b)$ and $n^{2}=m g / M \cdot(a+b) / a b$, approximately. The former root makes $B / A=(a+b) / a$, nearly, so that the two masses are always nearly in a line with the point of suspension, $m$ now oscillating like the bob of a pendulum of length $a+b$. In the second mode the ratio $B / A$ is small, so that $m$ is approximately at rest; the motion of $M$ is then like that of a particle attached to a string which is stretched between fixed points with a tension $m g$ (cf. $\S 6$ ).

Another case of interest is obtained if we make $a$ infinite. One root of (19) then vanishes, and the other is

$$
n^{2}=\frac{g}{(1-\mu) b}=\frac{g}{b}\left(1+\frac{m}{M}\right),
$$

which makes $A / B=-m / M$. This indicates that if the support of a simple pendulum yield horizontally, but without elasticity, the frequency is increased in a certain ratio which is of course

* The influence of dissipation is of course here neglected. If $m$ be subject to a frictional resistance, and especially if the modulus of decay be less than the period of the fluctuation given by the above theory, the phenomena are modified, and the illustration of the theory of resonance ( $\$ 12)$ is improved. There is now a continual, though possibly a slow, drain on the original energy of $M$. 
smaller the greater the inertia of the support. This is however more easily seen directly.

\section{General Equations of a Multiple System.}

The general theory of the small oscillations of a multiple system can only be given here in outline. In the case of one degree of freedom $(\$ 7)$ it was possible to base the theory on the equation of energy alone, but when we have more than one dependent variable this is no longer sufficient, and some further appeal must be made to Dynamics. For brevity of statement we will suppose that there are two degrees of freedom, but there is nothing in the argument which cannot at once be extended to the general case.

We imagine, then, a system such that every configuration which we need consider can be specified by means of two independent geometric variables or "coordinates" $q_{1}, q_{2}$. If in any configuration $\left(q_{1}, q_{2}\right)$ the coordinate $q_{1}$ (alone) receive an infinitesimal variation $\delta q_{1}$, any particle $m$ of the system will undergo a displacement $\delta s_{1}=\alpha_{1} \delta q_{1}$ in a certain direction. Similarly if $q_{2}$ alone be varied $m$ will be displaced through a space $\delta s_{2}=\alpha_{2} \delta q_{2}$ in a certain direction, different in general from the former. The resultant displacement $\delta s$ when both variations are made is given by

$$
\begin{aligned}
\delta s^{2} & =\delta s_{1}{ }^{2}+2 \delta s_{1} \delta s_{2} \cos \theta+\delta s_{2}{ }^{2} \\
& =\alpha_{1}{ }^{2} \delta q_{1}{ }^{2}+2 \alpha_{1} \alpha_{2} \cos \theta \delta q_{1} \delta q_{2}+\alpha_{2}{ }^{2} \delta q_{2}{ }^{2},
\end{aligned}
$$

where $\theta$ denotes the angle between the directions of $\delta s_{1}, \delta s_{2}$. If we divide by $\delta t^{2}$, we obtain the square of the velocity $v$ of the particle $m$, in any motion of the system through the configuration $\left(q_{1}, q_{2}\right)$, in terms of the generalized "components of velocity" $\dot{q}_{1}, \dot{q}_{2}$, thus

$$
v^{2}=\alpha_{1}^{2} \dot{q}_{1}^{2}+2 \alpha_{1} \alpha_{2} \cos \theta \dot{q}_{1} \dot{q}_{2}+\alpha_{2}^{2} \dot{q}_{2}^{2}
$$

The total kinetic energy of the system is therefore given by

where

$$
2 T=\Sigma\left(m v^{2}\right)=a_{11} \dot{q}_{1}^{2}+2 a_{12} \dot{q}_{1} \dot{q}_{2}+a_{22} \dot{q}_{2}^{2},
$$

$$
a_{11}=\Sigma\left(m x_{1}^{2}\right), \quad a_{12}=\Sigma\left(m \alpha_{1} \alpha_{2} \cos \theta\right), \quad a_{22}=\Sigma\left(m \alpha_{2}^{2}\right),
$$

the summation $\Sigma$ extending over all the particles $m$ of the 
system. The coefficients $a_{11}, a_{12}, a_{22}$ are in general functions of $q_{1}, q_{2}$; they are called the "coefficients of inertia" for the particular configuration considered.

Next, let $\boldsymbol{F}_{1}$ denote the total force acting on $m$, resolved in the direction of $\delta s_{1}$, and let $F_{2}$ have the corresponding meaning for the direction of $\delta s_{2}$. The work done on the system in any infinitesimal displacement will therefore be

$$
\Sigma\left(F_{1} \delta s_{1}+F_{2} \delta s_{2}\right)=\Sigma\left(F_{1} \alpha_{1}\right) \delta q_{1}+\Sigma\left(F_{2} \alpha_{2}\right) \delta q_{2} .
$$

If there are no extraneous forces, this work is accounted for by a diminution in the potential energy $V$ of the system. When extraneous forces act we have in addition the work due to these, which we may suppose expressed in the form

$$
Q_{1} \delta q_{1}+Q_{2} \delta q_{2} \text {. }
$$

The coefficients $Q_{1}, Q_{2}$ are called, by an obvious analogy, the generalized "components of (extraneous) force." Hence

$$
\Sigma\left(F_{1} \alpha_{1}\right) \delta q_{1}+\left(F_{2} \alpha_{2}\right) \delta q_{2}=-\delta V+Q_{1} \delta q_{1}+Q_{2} \delta q_{2},
$$

whence

$$
\left.\begin{array}{l}
\Sigma\left(F_{1} \alpha_{1}\right)=-\frac{\partial V}{\partial q_{1}}+Q_{1}, \\
\Sigma\left(F_{2} \alpha_{2}\right)=-\frac{\partial V}{\partial q_{2}}+Q_{2} .
\end{array}\right\}
$$

In the application to small oscillations we assume that $q_{1}, q_{2}$ are small quantities vanishing in the configuration of equilibrium, and for consistency we must also suppose that the disturbing forces $Q_{1}, Q_{2}$ are small. The quantities $\alpha_{1}, \alpha_{2}$ and therefore also $a_{11}, a_{12}, a_{22}$ may now be treated as constants. The velocity of the particle $m$ is made up of components $\alpha_{1} \dot{q}_{1}$, $\alpha_{2} \dot{q}_{2}$ in the directions $\delta s_{1}$ and $\delta s_{2}$, respectively; and if we neglect the squares of small quantities its acceleration is made up in like manner of components $\alpha_{1} \ddot{q}_{1}, \alpha_{2} \ddot{q}_{2}{ }^{*}$. Hence resolving in the direction of $\delta s_{1}$ the forces acting on $m$ we have

and similarly

$$
\left.\begin{array}{l}
m\left(\alpha_{1} \ddot{q}_{1}+\alpha_{2} \ddot{q}_{2} \cos \theta\right)=F_{1}, \\
m\left(\alpha_{1} \ddot{q}_{1} \cos \theta+\alpha_{2} \ddot{q}_{2}\right)=F_{2} .
\end{array}\right\}
$$

* The former of these two quantities is (to the first order) the acceleration calculated on the supposition that $q_{1}$ alone varies, and the latter is the acceleration when $q_{2}$ alone varies. It is only on the hypothesis of infinitely small motions that the resultant acceleration is obtained by superposition of these. 
If we multiply the former of these equations by $\alpha_{1}$ and the second by $\alpha_{2}$, and sum for all the particles of the system, we find, with the notation of (4),

$a_{11} \ddot{q}_{1}+a_{12} \ddot{q}_{2}=-\frac{\partial V}{\partial q_{1}}+Q_{1}$

and similarly

$$
\left.a_{21} \ddot{q}_{1}+a_{22} \ddot{q}_{2}=-\frac{\partial V}{\partial q_{2}}+Q_{2},\right\}
$$

where $a_{21}$ is of course identical with $a_{12}$.

When there are no extraneous forces these equations are by hypothesis satisfied by $\ddot{q}_{1}=0, \ddot{q}_{2}=0$. The configuration of equilibrium is therefore characterized by the property that

$$
\frac{\partial V}{\partial q_{1}}=0, \quad \frac{\partial V}{\partial q_{2}}=0,
$$

in other words, the potential energy is stationary for all infinitesimal displacements therefrom. Hence if $V$ be expanded in powers of $q_{1}, q_{2}$, the terms of the first order will be absent, and we may write with sufficient approximation

$$
2 V=c_{11} q_{1}^{2}+2 c_{12} q_{1} q_{2}+c_{22} q_{2}^{2},
$$

a constant term being omitted. The quantities $c_{11}, c_{12}, c_{22}$ are called the "coefficients of stability."

Hence (9) may be written

$$
\left.\begin{array}{l}
a_{11} \ddot{q}_{1}+a_{12} q_{2}+c_{11} q_{1}+c_{12} q_{2}=Q_{1} \\
a_{21} \ddot{q}_{1}+a_{22} \ddot{q}_{2}+c_{21} q_{1}+c_{22} q_{2}=Q_{2}
\end{array}\right\}
$$

where $c_{21}=c_{12}$.

If we look back to any of the special problems of $\S 14$ we shall recognize that the equations of motion are in fact of this type. For example, in the case of the double-pendulum we have

$$
2 T=M \dot{x}^{2}+m \dot{y}^{2}, \quad 2 V=M g \frac{x^{2}}{a}+m g\left\{\frac{x^{2}}{a}+\frac{(y-x)^{2}}{b}\right\} .
$$

The formulae therefore correspond if we put

$$
\left.\begin{array}{c}
q_{1}=x, \quad q_{2}=y, \\
a_{11}=M, \quad a_{12}=0, \quad a_{22}=m, \\
c_{11}=(M+m) g / a+m g / b, \quad c_{12}=-m g / b, \quad c_{22}=m g / b .
\end{array}\right\}
$$


The general case of $m$ degrees of freedom hardly differs except in the length of the formulae. We have then $m$ equations of the type

$$
a_{s 1} \ddot{q}_{1}+a_{82} \ddot{q}_{2}+\ldots+a_{s m} \ddot{q}_{m}+c_{81} q_{1}+c_{82} q_{2}+\ldots+c_{8 m} q_{m}=Q_{8},
$$

where $s$ is any one of the integers $1,2,3, \ldots m$.

\section{Free Periods of a Multiple System. Stationary}

\section{Property.}

In the case of free vibrations we have $Q_{1}=0, Q_{2}=0$, and the solution of $\S 15(12)$ then follows exactly the same course as in the particular examples already given. We assume

$$
q_{1}=A_{1} \cos (n t+\epsilon), \quad q_{2}=A_{2} \cos (n t+\epsilon)
$$

and obtain $\quad\left(c_{11}-n^{2} a_{11}\right) A_{1}+\left(c_{12}-n^{2} a_{12}\right) A_{2}=0$,

$$
\left.\left(c_{21}-n^{2} c_{21}\right) A_{1}+\left(c_{22}-n^{2} a_{22}\right) A_{2}=0 .\right\}
$$

Eliminating the ratio $A_{1}: A_{2}$, we obtain

$$
\left|\begin{array}{cc}
c_{11}-n^{2} a_{11}, & c_{12}-n^{2} a_{12} \\
c_{21}-n^{2} a_{21}, & c_{22}-n^{2} a_{22}
\end{array}\right|=0,
$$

where (it is to be noticed) the determinant is of the "symmetrical" type. This equation gives the two admissible values of $n^{2}$. Adopting either of these we obtain a solution in which the ratio of $A_{1}$ to $A_{2}$ is determined by either of the equations (2). The mode of vibration thus ascertained involves therefore two arbitrary constants, viz. the absolute value of (say) $A_{1}$, and the initial phase $\epsilon$. The second root of (3) leads to another solution of like character.

The extension of the method to the general case is obvious, but it may be well to state the results formally. In any conservative system of $m$ degrees of freedom there are in general $m$ distinct "normal modes" of free vibration about a configuration of stable equilibrium, the frequencies of which are given by a symmetrical determinantal equation of the $m$ th order in $n^{2}$, analogous to (3), and so depend solely on the constitution of the system. In each of these modes the system oscillates exactly as if it had only one degree of freedom, the coordinates $q_{1}, q_{2}, \ldots q_{m}$ being in constant ratios to one another, and the description of $\S 7$ therefore applies. The directions of motion of the various particles and the relative amplitudes are 
in any one mode determinate, though usually different for different modes, the only arbitrary elements being the absolute amplitude and the phase-constant.

The equations of motion being necessarily linear, since products and squares of the coordinates and their differential coefficients with respect to the time are expressly excluded, it follows that the different solutions may be superposed by addition of the corresponding expressions. This has been sufficiently illustrated in the preceding examples. By superposing in this way the $m$ normal modes, each with its arbitrary amplitude and phase, we obtain a solution involving $2 m$ arbitrary constants, which is exactly the right number to enable us to represent the effect of arbitrary initial values of the coordinates $q_{1}, q_{2}, \ldots q_{m}$ and velocities $\dot{q}_{1}, \dot{q}_{2}, \ldots \dot{q}_{m}$. In other words, the most general free motion of the system about a configuration of stable equilibrium may be regarded as made up of the $m$ normal modes with suitable amplitudes and initial phases. This principle dates from D. Bernoulli* (1741).

In particular cases it may happen that two (or more) of the natural periods of the system coincide. There is then a corresponding degree of indeterminateness in the character of the normal modes. The simplest example is furnished by the spherical pendulum, or by a particle oscillating in a smooth spherical bowl. The normal modes may then be taken to correspond to any two horizontal directions through the position of equilibrium. From the theoretical standpoint such coincidences may be regarded as accidental, since they are destroyed by the slightest alteration in the constitution of the system (e.g. if the bowl in the above illustration be in the slightest degree ellipsoidal), but in practice they often lead to interesting results. Cf. $§ 53$ below.

An important characteristic of the normal modes, first pointed out by Lord Rayleigh in 1883 , has still to be referred

* Daniel Bernoulli (1700-1782), one of the younger members of the distinguished family of Swiss mathematicians. Professor of mathematics at St Petersburg (1725-33), and of physics at Bâle (1750-82). His chief work was on hydrodynamics, on the theory of vibrating strings, and on the flexure of elastic beams. 
to. If, by the introduction of frictionless constraints which do no work, the system be restricted to vibrate in a mode only slightly different from one of these, the period will be altered only by a small quantity of the second order. In other words the periods of the several normal modes are "stationary" when compared with those of slightly different constrained modes. Suppose, for instance, that the normal mode in question is such that in it the coordinate $q_{1}$ alone varies. We have, then, in (2), $a_{12}=0, c_{12}=0$, and the natural frequency is determined by $n^{2}=c_{11} / a_{11}$. If the constraint be expressed by $q_{2}=\lambda q_{1}$, the condition that the constraining forces shall do no work, viz. $Q_{1} \dot{q}_{1}+Q_{2} \dot{q}_{2}=0$, or $Q_{1}+\lambda Q_{2}=0$, leads to

$$
\left(a_{11}+\lambda^{2} a_{22}\right) \ddot{q}_{1}+\left(c_{11}+\lambda^{2} c_{22}\right) q_{1}=0,
$$

and the speed $(p)$ is accordingly given by

$$
p^{2}=\frac{c_{11}+\lambda^{2} c_{22}}{a_{11}+\lambda^{2} a_{22}} \text {. }
$$

When $\lambda$ is small, this differs from $n^{2}$ by a small quantity of the second order. The proof, although limited to two degrees, is easily generalized. Owing to our liberty of choice of the coordinates, we can always arrange that $q_{1}$ shall be the only coordinate which varies in the mode in question, and that the constraint shall be expressed by a system of relations of the type $q_{2}=\lambda q_{1}, q_{3}=\mu q_{1}, q_{4}=\nu q_{1}, \ldots$.

For an obvious illustration we may have recourse again to the particle on a smooth surface. If the constrained path be a vertical section through the lowest point, the period is $2 \pi \sqrt{ }(R / g)$, where $R$ is the radius of curvature of the section, and it is known that $R$ is a maximum or minimum for the principal sections.

The equation (5) shews further that the constrained period is (as in the particular case) intermediate between the two natural periods; this property can also be generalized.

It follows that even when it is not easy to ascertain the precise character of a particular normal mode, a close approximation to the frequency can often be obtained on the assumption of an assumed type which we can judge on independent grounds to be a fairly good representation of the true one. And in the 
case of the gravest natural mode the frequency thus obtained will be an upper limit. Take, for instance, the case of three equal particles attached at equal intervals to a tense string ( $\$ 14$ ), and consider an assumed type of symmetrical vibration in which $x=z=\lambda y$. The kinetic energy is then given by

$$
2 T=M\left(\dot{x}^{2}+\dot{y}^{2}+\dot{z}^{2}\right)=M\left(1+2 \lambda^{2}\right) \dot{y}^{2},
$$

so that the inertia-coefficient is $M\left(1+2 \lambda^{2}\right)$. For the potential energy we have

$$
2 V=\frac{P}{a}\left\{x^{2}+(y-x)^{2}+(z-y)^{2}+z^{2}\right\}=\frac{P}{a}\left(4 \lambda^{2}-4 \lambda+2\right) y^{2},
$$

as is found by calculation of the work required to stretch the string (as in $\$ 22$ ), or otherwise. The coefficient of stability is therefore $P / a \cdot\left(4 \lambda^{2}-4 \lambda+2\right)$. For the speed $(p)$ we then have

$$
p^{2}=\frac{P}{M a} \cdot \frac{4 \lambda^{2}-4 \lambda+2}{1+2 \lambda^{2}} .
$$

This is stationary for $\lambda= \pm \frac{1}{2} \sqrt{ } 2$, and the corresponding speeds are as in $\S 14$. In this case it was evident beforehand that the assumed type would include the true natural modes of symmetrical character.

It is unnecessary for the purposes of this book to discuss in detail the theory of dissipation in a multiple system. The general effect is the same as in $\$ 12$; the free vibrations gradually die out, but if the dissipative forces be relatively small the periods are not sensibly affected.

\section{Forced Oscillations of a Multiple System. Prin-} ciple of Reciprocity.

The theory of forced oscillations is sufficiently illustrated if in $\$ 15$ (12) we assume that $Q_{1}$ varies as $\cos p t$, whilst $Q_{2}=0$. The equations will be satisfied if we assume that $q_{1}$ and $q_{2}$ both vary as $\cos p t$, provided

$$
\left.\begin{array}{l}
\left(c_{11}-p^{2} a_{11}\right) q_{1}+\left(c_{12}-p^{2} a_{12}\right) q_{2}=Q_{1}, \\
\left(c_{21}-p^{2} a_{21}\right) q_{1}+\left(c_{22}-p^{2} a_{22}\right) q_{2}=0 .
\end{array}\right\}
$$

These determine the (constant) ratios of $q_{1}$ and $q_{2}$ to $Q_{1}$; thus

$$
q_{1}=\frac{c_{22}-p^{2} a_{22}}{\Delta\left(p^{2}\right)} \cdot Q_{1}, \quad q_{2}=-\frac{c_{12}-p^{2} a_{12}}{\Delta\left(p^{2}\right)} \cdot Q_{1},
$$


where $\Delta\left(p^{2}\right)$ is the determinant on the left-hand side of $\S 16$ (3), with $p^{2}$ written for $n^{2}$. The general conclusion is that when a periodic force of simple-harmonic type acts on any part of the system, every part will execute a simple-harmonic vibration of the same period, with synchronism of phase, but the amplitude will of course be different in different parts. When the period of the forced vibration nearly coincides with that of one of the free modes, an abnormal amplitude of forced vibration will in general result, owing to the smallness of the denominator in the formulae (2). For a complete account of this matter we should have to take dissipative forces into consideration, as in $\S 12$.

A remarkable theorem of reciprocity, first proved by Helmholtz for aerial vibrations, and afterwards greatly extended by Lord Rayleigh, follows from (2). If we imagine a second case of forced vibration (distinguished by accents) in which $Q_{1}{ }^{\prime}=0$ whilst $Q_{2}^{\prime}$ varies as $\cos p t$, we shall have

$$
q_{1}^{\prime}=-\frac{c_{21}-p^{2} a_{21}}{\Delta\left(p^{2}\right)} \cdot Q_{2}^{\prime}
$$

Comparing with (2), we see that

$$
q_{2}: Q_{1}=q_{1}^{\prime}: Q_{2}^{\prime} \text {. }
$$

The interpretation is most easily expressed when the "forces" $Q_{1}$ and $Q_{2}^{\prime}$ are of the same character, e.g. both ordinary statical forces, or both couples, in which case we may put $Q_{1}=Q_{2}{ }^{\prime}$, and obtain $q_{2}=q_{1}^{\prime}$. In words: The vibration of type 2 due to a given periodic force of type 1 agrees in amplitude and phase with the vibration of type 1 due to an equal force of type 2 . An example from the theory of strings will be found in $\$ 28$. The above proof is easily extended to the general case of $m$ degrees of freedom.

18. Composition of Simple-Harmonic Vibrations in Different Directions.

We recur to the subject of composition of simple-harmonic vibrations which, though not so important as in Optics, claims a little further attention. If in a freely vibrating system we fix our attention on a particular particle, the directions in which it 
oscillates in the several normal modes will in general be different. The superposition then takes place of course according to the law of geometrical or vector addition.

It will suffice to consider the case of two degrees of freedom, where we have independent simple-harmonic vibrations in the directions corresponding to the $\delta s_{1}, \delta s_{2}$ of $\S 15$. The result is a plane orbit, usually of a complicated character. For instance, in the case of Blackburn's pendulum ( $\$ 14)$, we have

$$
x=A \cos \left(n_{1} t+\epsilon_{1}\right), \quad y=B \cos \left(n_{2} t+\epsilon_{2}\right),
$$

where $x, y$ are rectangular coordinates. The orbit is here contained within the rectangle bounded by the lines $x= \pm A$, $y= \pm B$. If $n_{1}, n_{2}$ are commensurable, the values of $x, y$ and $\dot{x}, \dot{y}$ will recur after the lapse of an interval equal to the least common multiple of the two periods, and the path will be re-entrant. The resulting figures, obtained in this and in other ways, are associated with the name of Lissajous*, who has had many followers in a region which is very attractive from the experimental point of view.

The simplest case is that of $n_{1}=n_{2}$. If we eliminate $t$ in (1) we then obtain

$$
\frac{x^{2}}{A^{2}}-\frac{2 x y}{A B} \cos \left(\epsilon_{1}-\epsilon_{2}\right)+\frac{y^{2}}{B^{2}}=\sin ^{2}\left(\epsilon_{1}-\epsilon_{2}\right) .
$$

This represents an ellipse which, if the initial phases $\epsilon_{1}, \epsilon_{2}$ coincide, or differ by $\pi$, degenerates into a straight line (Fig. 20). The simplest mechanical illustration is furnished by the spherical pendulum. When the relation is that of the octave $\left(n_{1}=2 n_{2}\right)$ we have a curve with two loops, which may degenerate into one or other of two parabolic arcs (Fig. 21). The curves in these and in other cases of commensurability are easily traced from the formulae (1) with the help of tables. A simple geometrical construction is indicated in Fig. 22, where the circumferences of the auxiliary circles are divided into segments corresponding to equal intervals of time in the two simple-harmonic motions which are to be compounded. If we start at a corner of any

* J. A. Lissajous (1822-80). Professor of physics at the Lycée St Louis 1850-74; rector of the Academy of Chambery $1874-5$, and of Besançon 1875-9. His chief memoir, Étude optique des mouvements vibratoires, was published in 1873. 

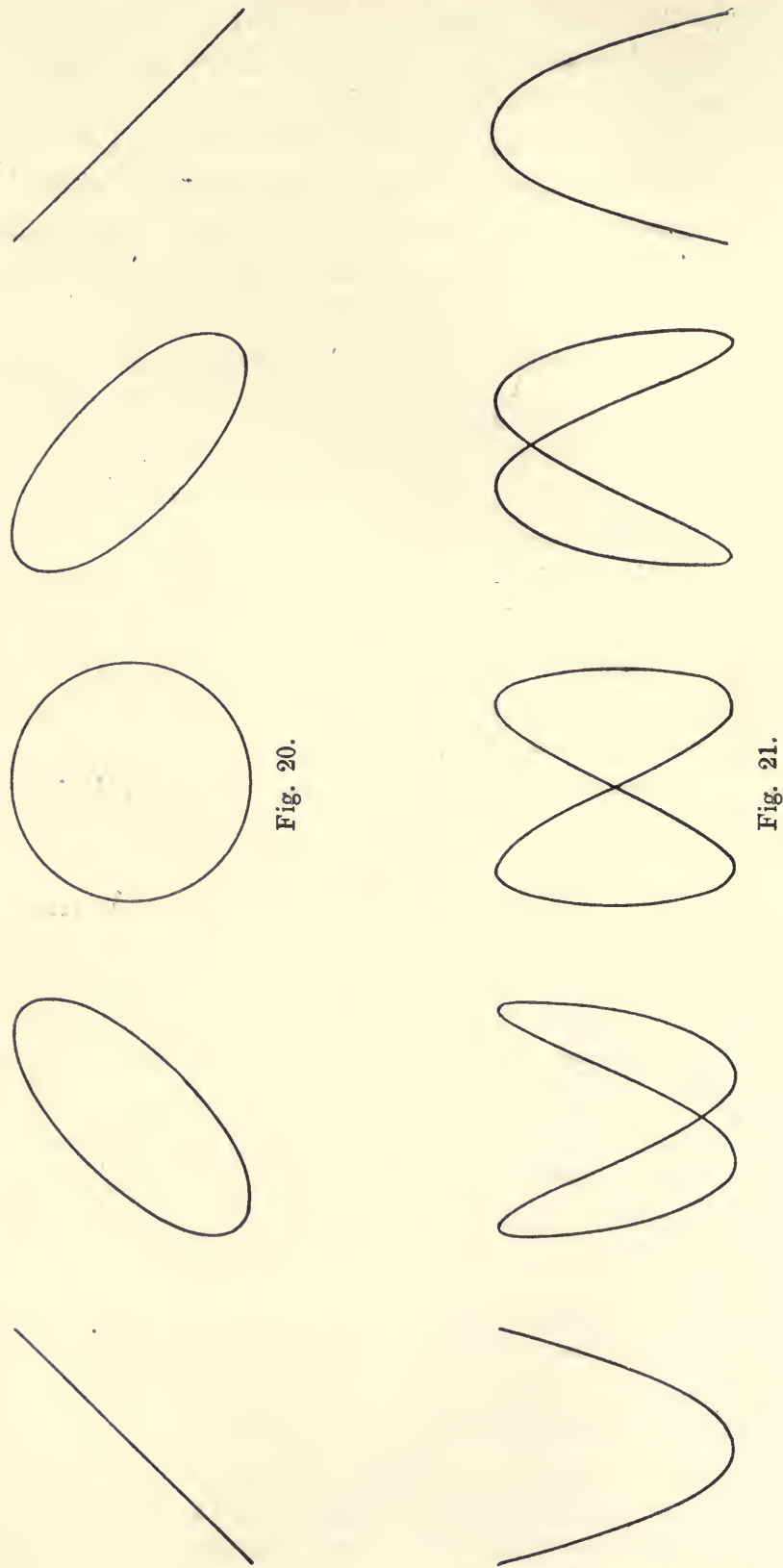
one of the rectangles in the figure, and proceed diagonally, we pass through a succession of points, equidistant in time, on a curve of the system.

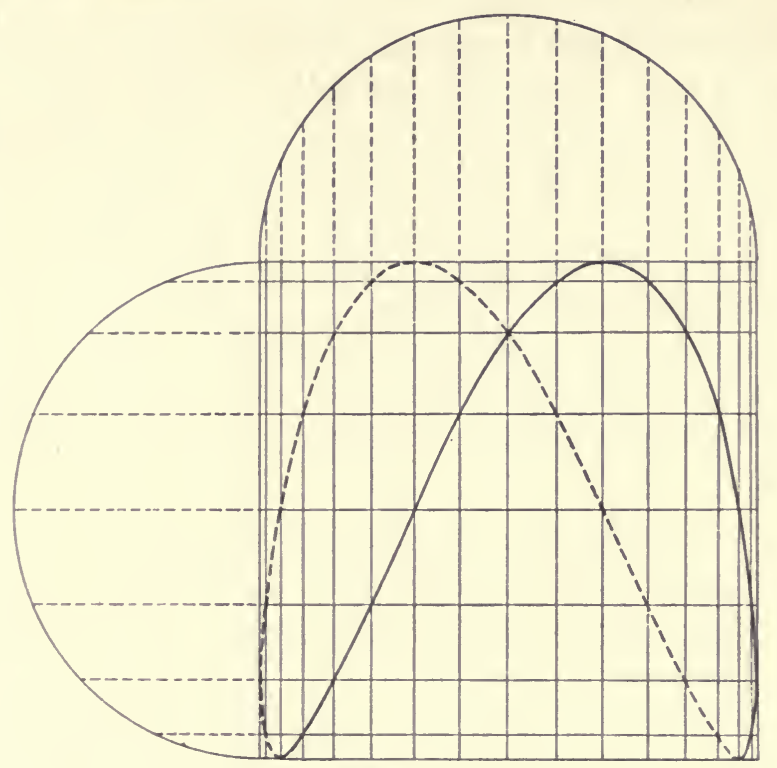

Fig. 22.

Another conception of these figures, also due to Lissajous, may be mentioned. If we write $\theta$ for $n_{1} t$, and adjust the origin of $t$, the formulae (1) are equivalent, on the hypothesis of commensurability, to

$$
x=a \cos \theta, \quad y=b \cos \frac{p}{q}(\theta-\alpha),
$$

where $p / q$ is a fraction in its lowest terms. These equations, when combined with

$$
z=a \sin \theta
$$

represent a curve of sines traced on the surface of the circular cylinder

$$
x^{2}+z^{2}=a^{2}
$$

and going through its period $p$ times in $q$ successive circuits of the cylinder. The Lissajous curve (3) is the orthogonal projection of this curve on a plane $(z=0)$ through the axis of 
the cylinder. This is illustrated by Fig. 22, where the dotted branch may be regarded as the projection of that part of the sine-curve which lies on the rear half of the curved surface. A change in the relative phase in (1) is equivalent to a change in the angle $\alpha$, and may be represented by a rotation of the cylinder about its axis, of corresponding amount. This, again, may be illustrated from Fig. 22 by starting the curve one step further to the right or left. When the ratio of the periods is nearly, but not exactly, that of two integers, the orbit gradually passes through the various phases of the commensurable case, in a recurring cycle*. Thus in the case of approximate unison, or approximate octave, the cycle includes the phases shewn in Fig. 21 or 22 , followed by the same in reverse order. The same result is obtained by a continuous rotation of Lissajous' cylinder.

\section{Transition to Continuous Systems.}

The space which we have devoted to the study of dynamical systems of finite freedom is justified by the consideration that we here meet with principles, in their primitive and most easily apprehended forms, which run through the whole of theoretical acoustics. In the subsequent chapters we shall be concerned with systems such as strings, bars, membranes, columns of air, where the number of degrees of freedom is infinite. Mathematically, it is sometimes possible to pass from one of these classes to the other by a sort of limiting process, as when D. Bernoulli (1732) discussed the vibrations of a hanging chain as a limiting form of the problem where a large number of equal and equidistant particles are attached to a tense string whose own mass is neglected. In any case, there can be no question that the general principles referred to retain their validity. The main qualification to be noticed is that the normal modes are now infinite in number. It is usual to consider them as arranged

* In Lissajous' method the vibrations which are optically compounded are those of two tuning forks. The figures obtained when the tones sounded by the forks form any one of the simpler musical intervals give a beautiful verification of the numerical relations referred to in $\S 3$. In the case of unison, when the tuning is not quite exact, the cycle of changes synchronises with the beats which are heard; see $\S 10$. 
in ascending order of frequency; the mode of slowest vibration may still be called the "fundamental," and is generally the most important.

Before leaving the general theory it may be desirable to emphasize once more the importance of the simple-harmonic type of vibration from the dynamical point of view. We have seen that it is the characteristic type for a frictionless system of one degree of freedom, or (more generally) for a system oscillating as if it possessed only one degree, as in the case of the normal modes. It is also the only type of imposed vibration which is accurately reproduced, on a larger or smaller scale, in every part of the system. If a force of perfectly arbitrary type act at any point, the vibrations produced in other parts of the system have as a rule no special resemblance to this or to one another; it is only in the case of a periodic force following the simpleharmonic law of variation with the time that the induced vibrations are exactly similar, and keep step with the force. Moreover it is only in so far as the disturbing force is simpleharmonic, or contains simple-harmonic constituents, that it is capable of generating a forced vibration of abnormal amplitude when a critical frequency is approached. It is in these circumstances that Helmholtz found the clue to his theory of audition, to which we shall have to refer at a later stage.

\section{On the Use of Imaginary Quantities.}

The treatment of dynamical equations can often be greatly simplified by the use of so-called "imaginaries." As we shall occasionally have recourse to this procedure, it may be convenient to explain briefly the principles on which it rests.

The reader will be familiar with the geometrical representation of a "complex" quantity $a+i b$, where $a, b$ are real and $i$ stands for $\sqrt{ }(-1)$, by a vector drawn from the origin to the point whose rectangular coordinates are $(a, b)$, and with the fact that addition of imaginaries corresponds to geometrical addition (or composition) of the respective vectors. The symbol $a+i b$ when applied as a multiplying operator to any vector denotes the same process by which the vector $a+i b$ may be supposed to have been derived from the vector 1 , viz. it 
alters the length in a certain ratio $r$, and turns it through a certain angle $\alpha$. These quantities are defined by

$$
r \cos \alpha=a, \quad r \sin \alpha=b,
$$

or

$$
r=\sqrt{ }\left(a^{2}+b^{2}\right), \quad \alpha=\tan ^{-1}(b / a),
$$

the quadrant in which $\alpha$ lies being determined by the sign attributed to $\cos \alpha$ or $\sin \alpha$ by (1). We have then

$$
a+i b=r(\cos \alpha+i \sin \alpha) \text {. }
$$

Hence a symbol of the form $\cos \alpha+i \sin \alpha$ denotes the operation of turning a vector through an angle $\alpha$ without alteration of length; in particular the symbol $i$ denotes the operation of turning through a right angle in the positive (counter-clockwise) direction.

The symbol

$$
w=\cos \theta+i \sin \theta
$$

may be represented by a unit vector $O P$ drawn from $O$ in the direction $\theta$. If we regard this as a function of $\theta$, and if

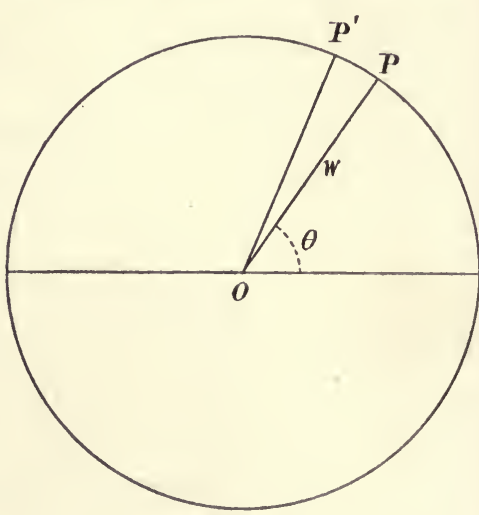

Fig. 23. $w+\delta w$ be represented by $O P^{\prime}$, the angle $P O P^{\prime}$ will be equal to $\delta \theta$. The vector $P P^{\prime}$ which represents $\delta w$ will therefore have a length $\delta \theta$, and since it is turned through a right angle relatively to $O P$, its symbol will be $i \delta \theta . w$. Hence

$$
\frac{d w}{d \theta}=i w \text {. }
$$

It is easily shewn that the only solution of this equation which fulfils the necessary condition that $z=1$ for $\theta=0$, is

$$
w=e^{i \theta},
$$

where $e^{i \theta}$ is to be taken as defined by the ordinary exponential series. Thus

$$
e^{i \theta}=\cos \theta+i \sin \theta .
$$

We may add that the "addition-theorem" of the exponential function can now be derived immediately from the geometrical representation. 
It has been thought worth while to recapitulate these elementary matters because they have interesting illustrations in the present subject. Thus if $x, y$ be rectangular coordinates, and we write

$$
\begin{aligned}
& z=x+i y, \\
& z=C e^{i n t}
\end{aligned}
$$

the equation

where $C$ may of course be complex, expresses that the vector $C$ is turned in a time $t$ through an angle $n t$ in the positive direction. It therefore represents uniform motion in a circle, with angular velocity $n$, in the positive direction. The radius of this circle is given by the "absolute value" of $C$, which is often denoted by $|C|$; thus if $C=A+i B$, where $A$ and $B$ are real, we have $|C|=\sqrt{ }\left(A^{2}+B^{2}\right)$. In the same way the equation

$$
z=C^{\prime} e^{-i n t}
$$

represents uniform motion in a circle, with angular velocity $n$, in the negative (or clockwise) direction.

We come now to the application to linear differential equations with constant coefficients. From our point of view the simplest case is the equation

$$
\frac{d^{2} x}{d t^{2}}+n^{2} x=0
$$

of $\S 4$. In order that every step of the work may admit of interpretation, we associate with this the independent equation

$$
\frac{d^{2} y}{d t^{2}}+n^{2} y=0
$$

as in the theory of the spherical pendulum. The two may be combined in the one equation

$$
\frac{d^{2} z}{d t^{2}}+n^{2} z=0
$$

which may indeed be regarded as representing directly, without the intermediary of (11) and (12), the law of acceleration in the spherical pendulum and similar problems. To solve (13) we assume $z=C e^{\lambda t}$, and we find that the equation is satisfied provided $\lambda^{2}+n^{2}=0$, or $\lambda= \pm i n$. Since different solutions can be added, we obtain the form

$$
z=C e^{i n t}+C^{\prime} e^{-i n t},
$$


with two complex arbitrary constants $C, C^{\prime}$. These can be determined so as to identify $z$ and $\dot{z}$, at the instant $t=0$, with the vectors which represent the initial position and velocity of the point $(x, y)$. It appears from (14) that the most general motion of a point subject to (13) may be obtained by the superposition of two uniform circular motions in opposite directions. The same problem (virtually) has been treated in $\S 18$, where the path was found to be an ellipse. This resolution of an "elliptic harmonic" vibration into two circular vibrations in opposite directions has important applications in Optics.

The solution of the equation (11) may be derived from (14) by taking the "real" part of both sides, i.e. by projecting the motion on to the axis of $x$. Since $C, C^{\prime}$ are of the forms

$$
C=A+i B, \quad C^{\prime}=A^{\prime}+i B^{\prime},
$$

it might appear at first that the result would involve four arbitrary constants. These occur, however, in such a way that they are really equivalent only to two. Thus we find

$$
x=\left(A+A^{\prime}\right) \cos n t-\left(B-B^{\prime}\right) \sin n t .
$$

The kinematical reason for this is that, as regards their projections on a straight line, right-handed and left-handed circular motions are indistinguishable. An important practical corollary follows. We should have obtained equal generality, so far as the solution of (11) is concerned, if we had contented ourselves with either solution of (13), for example

and taken the real part

$$
z=C e^{i n t}
$$

$$
x=A \cos n t-B \sin n t .
$$

This conclusion is obviously not restricted to the particular differential equation (11) with which we started. The use of an adjunct equation such as (12) has only been resorted to in order to remove the suspicion of anything that can truly be called "imaginary" in the work. Such assistance can always be invoked mentally, but it is as unnecessary as it would be tedious always formally to introduce it. If in any case of a linear differential equation between $x$ and $t$, with constant real coefficients, we seek for a solution of the type $x=C e^{\lambda t}$, the imaginary values (if any) of $\lambda$ will occur in 
conjugate pairs of the form $m \pm i n$, and we may assert that the part of the solution corresponding to this pair of roots will be given with sufficient generality if we make use of one only of these, writing, for instance,

$$
x=C e^{(m+i n) t},
$$

and taking the real part.

We may apply these considerations, for example, to the equation

$$
\frac{d^{2} x}{d t^{2}}+k \frac{d x}{d t}+\mu x=0
$$

of resisted motion about an equilibrium position ( $\$ 11)$. If we put $x=C e^{\lambda t}$, we have

$$
\begin{aligned}
& \lambda^{2}+k \lambda+\mu=0 . \\
& \lambda=-\frac{1}{2} k \pm i n^{\prime}, \\
& n^{\prime}=\sqrt{ }\left(\mu-\frac{1}{4} k^{2}\right),
\end{aligned}
$$

provided $k^{2}<4 \mu$. On the above principle a sufficient solution is

or, in real form,

$$
x=C e^{-\frac{1}{2} k t+i n^{\prime} t},
$$

$$
x=e^{-\frac{1}{2} k t}\left(A \cos n^{\prime} t-B \sin n^{\prime} t\right),
$$

which is equivalent to $§ 11$ (8).

The same method can be followed with regard to the equation of forced oscillations, say

$$
\frac{d^{2} x}{d t^{2}}+k \frac{d x}{d t}+\mu x=f \cos p t .
$$

Instead of this we take the equation

$$
\frac{d^{2} z}{d t^{2}}+k \frac{d z}{d t}+\mu z=f e^{i p t}
$$

the implied adjunct equation being of the type (25) with $f \sin p t$ instead of $f \cos p t$ on the right hand. A particular solution is

provided

$$
\begin{gathered}
z=C e^{i p t}, \ldots . \\
\left(\mu-p^{2}+i k p\right) C=f . \\
z=\frac{f e^{i p t}}{\mu-p^{2}+i k p}
\end{gathered}
$$

Hence 
If we put

$$
\mu-p^{2}=R \cos \alpha, \quad k p=R \sin \alpha,
$$

this becomes

$$
z=\frac{f}{R} e^{i(p t-a)},
$$

the real part of which is

$$
x=\frac{f}{R} \cos (p t-\alpha) .
$$

This may be compared, for brevity, with the process of $\S 12$.

\section{Historical note.}

The theory of vibrations has a long and rather intricate history, in which Pure Mathematics and Mechanics have reacted on one another with great advantage to the progress of both sciences. Various special problems of great interest had been solved by the Bernoullis, Euler*, and other mathematicians, but it is to Lagrange that we owe the general theory of the small oscillations of a system of finite freedom treated by means of generalized coordinates. The work of Lagrange was purposely somewhat abstract in form ${ }_{+}^{+}$; the full dynamical interpretation was reserved for Thomson and Tait (Natural Philosophy, 1867), to whom we also owe the now current terminology of the subject. The theory has been very greatly extended by Lord Rayleigh, and systematically applied to acoustics as well as other branches of physics, in various writings, most of which (down to the year 1896) are incorporated in his Theory of Sound .

* Leonhard Euler, born at Bâle 1707, died at St Petersburg 1783. He wrote extensively on most branches of mathematics and mechanics, and fixed to a great extent the notations now in use.

+ Joseph Louis Lagrange, born at Turin 1736, died at Paris 1813, " the greatest mathematician since the time of Newton."

$\ddagger$ "On ne trouvera point de Figures dans cet Ouvrage. Les méthodes que j'y expose ne demandent ni constructions, ni raisonnemens géométriques ou méchaniques, mais seulement des opérations algébriques, assujéties à une marche régulière et uniforme." (Preface to the Mécanique Analytique, 1788.)

$\S 1$ st ed. London 1877,2 nd ed. London $1894-6$. See also his Scientific Papers, Cambridge 1899-1902. 


\title{
CHAPTER II
}

\author{
STRINGS
}

\section{Equation of Motion. Energy.}

We proceed to the more or less detailed study of the vibrations of various types of continuous systems. Amongst these the first place must for many reasons be assigned to the transverse vibrations of a uniform tense string. Historically; this was the first problem of the kind to be treated theoretically. The mathematical analysis is simple, and various points of the general theory sketched in the preceding chapter receive interesting illustrations, which are moreover easily verified experimentally. Again, the sequence of the natural periods of free vibration has the special "harmonic" relation which has long been recognized as in some way essential to good musical quality, although the true reason, which is ultimately a matter of physiology, has only in recent times been investigated. The mathematical theory has further suggested some remarkable theorems, as to the resolution of a vibration of arbitrary type into simple-harmonic constituents, which are of far-reaching significance. Finally it is to be noted that in the propagation of a disturbance along a uniform string we have the first and simplest type of wave-motion.

The string is supposed to be of uniform line-density $\rho$, and to be stretched with a tension $P$. The axis of $x$ is taken along the equilibrium position, and we denote by $y$ the transverse deflection at the point $x$, at time $t$. It is assumed that the gradient $\partial y / \partial x$ of the curve formed by the string at any instant is so small that the change of tension may be 
neglected. Under these conditions the equation of motion of an element $\delta x$ is

$$
\rho \delta x \cdot \frac{\partial^{2} y}{\partial t^{2}}=\delta(P \sin \psi),
$$

where $\psi$ denotes the inclination of the tangent line to the axis of $x$. The right-hand side is, in fact, the difference of the tensions on the two ends of the element, when resolved in the direction of $y$. In virtue of the assumption just made we may write $\sin \psi=\tan \psi=\partial y / \partial x$, so that (1) becomes

$$
\frac{\hat{o}^{2} y}{\partial t^{2}}=c^{2} \frac{\partial^{2} y}{\partial x^{2}}
$$

where

$$
c^{2}=P / \rho \text {. }
$$

It is easily seen that the constant $c$ has the dimensions of a velocity.

The kinetic energy of any portion of the string is given by

$$
T=\frac{1}{2} \rho \int \dot{y}^{2} d x
$$

taken between the proper limits. The potential energy may be calculated in two ways. In the first place we may imagine the string to be brought from rest in its equilibrium position to rest in any assigned form by means of lateral pressures applied to it. For simplicity suppose that at any stage of the process the ordinates all bear the same ratio $(k)$ to their final values $y$, so that the successive forms assumed by the string differ only in amplitude. The force which must be applied to an element $\delta x$ to balance the tensions on its ends is

$$
-\frac{\partial}{\partial x}(P \sin \psi) \delta x
$$

where $\sin \psi$ is now to be equated to $k \partial y / \partial x$; and the displacement when $k$ increases by $\delta k$ is $y \delta k$. The total work done on this element is therefore

$$
-P y y^{\prime \prime} \delta x \cdot \int_{0}^{1} k d k=-\frac{1}{2} P y y^{\prime \prime} \delta x,
$$

where the accents indicate differentiations with respect to $x$. The potential energy is accordingly

$$
V=-\frac{1}{2} P \int y y^{\prime \prime} d x \text {. }
$$


In the alternative method we calculate the work done in stretching the string against the tension $P$. The increase in length of an element $\delta x$ is

$$
\sqrt{ }\left(1+y^{\prime 2}\right) \delta x-\delta x=\frac{1}{2} y^{\prime 2} \delta x,
$$

approximately, so that,

$$
V=\frac{1}{2} P \int y^{\prime 2} d x
$$

The formulae (5), (6) lead to identical results when applied to the whole disturbed extent of the string. For by a partial integration we have

$$
-\int y y^{\prime \prime} d x=-\left[y y^{\prime}\right]+\int y^{\prime 2} d x
$$

where the first term refers to the limits. It vanishes at the extremities of the disturbed portion, since $y$ is there $=0$.

\section{Waves on an Unlimited String.}

The solution of $\S 22(2)$ is

$$
y=f(c t-x)+F(c t+x),
$$

where the functions $f, F$ are arbitrary. It is easily verified by differentiation that this formula does in fact satisfy the differential equation, and we shall see presently that by means of the two arbitrary functions which it contains we are able to represent the effect of any given initial distribution of displacement $(y)$ and velocity $(\dot{y})$. It was published by d'Alembert* in 1747 .

The two terms in (1) admit of simple interpretations. Taking the first term alone, we see that so far as this is concerned the value of $y$ is unaltered when $x$ and ct are increased by equal amounts; the displacement therefore which exists at the instant $t$ at the point $x$ is found at a later instant $t+\tau$ in the position $x+c \tau$. Hence the equation

$$
y=f(c t-x)
$$

represents a wave-form travelling unchanged with the velocity $c$ in the direction of $x$-positive. The equation

$$
y=F(c t+x)
$$

represents in like manner a wave travelling with the same velocity in the direction of $x$-negative. And it appears that

* J. le Rond d'Alembert (1717-83), encyclopaedist and mathematician; he made important contributions to dynamics and hydrodynamics. 
the most general free motion of the string may be regarded as made up of two such wave-systems superposed.

The form of the expression $\sqrt{ }(P / \rho)$ for the wave-velocity is to be noticed. As in all analogous cases the wave-velocity appears as the square root of the ratio of two quantities, one of which represents (in a.general sense) the elasticity, and the other the inertia, of the medium concerned.

A simple proof of the formula for the wave-velocity has been given by Prof. Tait*. Imagine a string to be drawn with constant velocity $v$ through a smooth curved tube, the portions outside the tube being straight and in the same line. Since there is no tangential acceleration the tension $P$ is uniform. Also the resultant of the tensions on the ends of an element $\delta s$, at any point of the tube, will be a force $P \delta s / R$ in the direction of the normal, where $R$ is the radius of curvature. This will balance the "centrifugal force" $\rho \delta s . v^{2} / R$ if $v^{2}=P / \rho$. Under this condition the tube may be abolished, since it exerts no pressure, and we have a standing wave on a moving string. If we now impress on everything a velocity $v$ in the opposite direction to the former, we have a wave progressing without change of form, on a string which is otherwise at rest, with the velocity $\sqrt{ }(P / \rho)$. It will be noticed that this investigation does not require the displacements to be small.

The motion of an unlimited string consequent on arbitrary initial conditions

$$
y=\phi(x), \quad \dot{y}=\psi(x), \quad[t=0],
$$

may be deduced from (1), but it will be sufficient to write down the result, viz.

$$
y=\frac{1}{2}\{\phi(x-c t)+\phi(x+c t)\}+\frac{1}{2} \int_{x-c t}^{x+c t} \psi(z) d z .
$$

This may be immediately veritied.

If the initial disturbance be restricted to a finite extent of the string, the motion finally resolves itself into two distinct waves travelling without change in opposite directions. In these separate waves we have

$$
\dot{y}=\mp c y^{\prime},
$$

"Encyc. Brit. 9th ed. Art. "Mechanics." 
as is seen at once by considering two consecutive positions of the wave-form. Thus if in Fig. 24 the curves $A, B$ represent the positions at the instants $t, t+\delta t$, we have $P Q=c \delta t, R P=-\dot{y} \delta t$, $R P / P Q=y^{\prime}$, whence the former of the relations (6). The same thing follows of course from differentiation of (2). Conversely, it is easily seen from (5), or otherwise, that if the

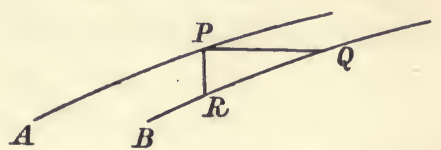

Fig. 24. initial conditions be adjusted so that either of the relations (6) is everywhere satisfied, a single progressive wave will result.

When the string is started with initial displacement, but no initial velocity, the formula (5) reduces to

$$
y=\frac{1}{2}\{\phi(x-c t)+\phi(x+c t)\} \text {. }
$$

The two component wave-forms resemble the initial profile, but are of half the height at corresponding points. It is easily seen without analysis that this hypothesis satisfies the condition of zero initial velocity.

It appears from (6) that in any case of a single progressive wave the expressions (4) and (6) of $\$ 22$ for the kinetic and potential energies are equal. Lord Rayleigh has pointed out that this very general characteristic of wave motion may be inferred otherwise as follows. Imagine the wave as resulting from an initial condition in which the string was at rest, and the energy $E$ therefore all potential, in the manner just explained. The two derived waves have half the amplitude (at corresponding points) of the original form, and the potential energy of each is therefore $\frac{1}{4} E$. Since the total energy of each wave must be $\frac{1}{2} E$, it follows that the kinetic energy of each must be $\frac{1}{4} E$.

In mathematical investigations it is not unusual to find the effect of dissipation represented by the hypothesis that each element of the string is resisted by a force proportional to its velocity, so that the differential equation takes the form

$$
\frac{\partial^{2} y}{\partial t^{2}}=c^{2} \frac{\partial^{2} y}{\partial x^{2}}-k \frac{\partial y}{\partial t} \text {. }
$$

As regards the theory of stringed instruments this particular 
correction has no importance, the direct influence of the air being quite insignificant; but the solution of (8) when $k$ is small is of some interest from the standpoint of wave-theory, and may therefore find a place here. If the square of $k$ be neglected, the equation may be written

$$
\frac{\partial^{2}}{\partial t^{2}}\left(y e^{\frac{1}{2} k t}\right)=c^{2} \frac{\partial^{2}}{\partial x^{2}}\left(y e^{\frac{1}{3} k t}\right) .
$$

This is of the same form as $\S 22(2)$, and therefore

$$
y=e^{-\frac{1}{2} k t} f(c t-x)+e^{-\frac{1}{2} k t} F(c t+x) \text {. }
$$

This represents two wave-systems travelling in opposite directions with velocity $c$; but there is now a gradual diminution of amplitude in each case as time goes on, as is indicated by the exponential factor. Again, since the functions are arbitrary, we may replace $f(c t-x)$ and $F^{\prime}(c t+x)$ by

$$
e^{\frac{1}{2} k(t-x / c)} f(c t-x) \text { and } e^{\frac{1}{2} k(t+x / c)} F^{\prime}(c t+x),
$$

respectively, so that the solution may also be written

$$
y=e^{-\frac{1}{2} k x / c} f(c t-x)+e^{\frac{1}{2} k x / c} F(c t+x) .
$$

This form is appropriate when a prescribed motion is maintained at a given point of the string. Thus if the imposed condition be that $y=\phi(t)$ for $x=0$, the waves propagated to the right of the origin are given by

$$
y=e^{-\frac{1}{2} k x / c} \phi\left(t-\frac{x}{c}\right) .
$$

The exponential shews the decrease of amplitude as the waves reach portions of the string further and further away from the origin.

\section{Reflection. Periodic Motion of a Finite String.}

If a point of the string, say the origin $O$, be fixed, we must have $y=0$ at this point for all values of $t$. Hence, in $§ 23(1)$,

$$
f(c t)+F(c t)=0 \text {, or } F(z)=-f(z) .
$$

The solution therefore takes the form

$$
y=f(c t-x)-f(c t+x)
$$

As applied for example to the portion of the string which lies to the left of $O$, this indicates the superposition of a direct 
When the initial data are of displacement only, i.e. with zero initial velocity, the successive forms assumed by the string in the course of a period can be obtained by a graphical construction. We suppose the initial form $y=\phi(x)$, where $\phi(x)$ is originally defined only for values of $x$ ranging from 0 to $l$, to be continued indefinitely both ways, subject to the conditions

$$
\phi(-x)=-\phi(x), \quad \phi(l+x)=-\phi(l-x) .
$$

If we imagine curves of the type thus obtained to travel both ways with velocity $c$, and if we take at each instant the arithmetic mean of the ordinates, in accordance with $\S 23(7)$, it is evident that the varying form thus obtained will represent

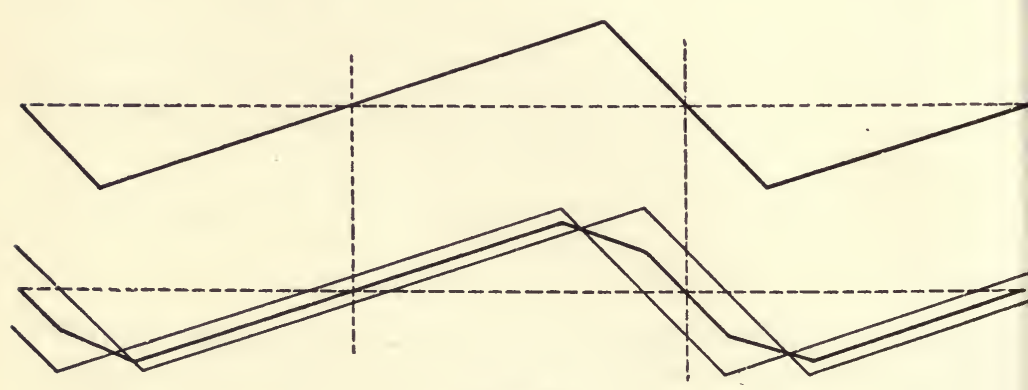

Fig. 26.

a possible motion on an unlimited string, in which the points $x=0, x= \pm l, x= \pm 2 l, \ldots$ remain at rest. The portion between $x=0$ and $x=l$ will therefore satisfy all the conditions of the question. The process is illustrated in the annexed Fig. 26; the initial form here consists of two straight pieces meeting at an angle, and the result after an interval $l / 8 c$ is ascertained.

In this way we might trace (after Young) the successive forms assumed by a string excited by "plucking," one point of the string being pressed aside out of its equilibrium position, and then released from rest, but the actual construction can in such a case be greatly simplified. It is easily seen that the form of the string at any instant consists in general of three portions; the outer portions have the same gradients as the two pieces into which the string was initially divided, whilst the gradient of the middle portion is the arithmetic mean of 
these, account being taken of sign. The line of this middle portion moves parallel to itself, with constant velocity, backwards and forwards between the two corners of the parallelogram of which the initial form constitutes two adjacent sides.

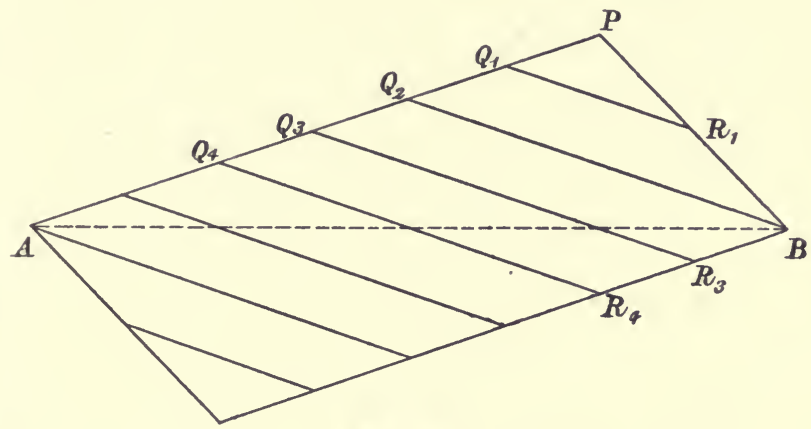

Fig. 27.

In the annexed Fig. 27, which corresponds with Fig. 26, the plucking is supposed to take place at a distance of one-fourth the length from one end, and the phases shewn follow one another at intervals of one-sixteenth of a complete period, the successive forms being $A P B, A Q_{1} R_{1} B, A Q_{2} B, A Q_{3} R_{3} B, A Q_{4} R_{4} B$, and so on. It is evident on inspection of the figure that any point of a plucked string moves backwards and forwards with constant velocity between two extreme positions, in which it rests alternately during (in general) unequal intervals. The space-time diagrams of the middle point, and of the point plucked, under the conditions of Fig. 27, are given in Fig. 28.
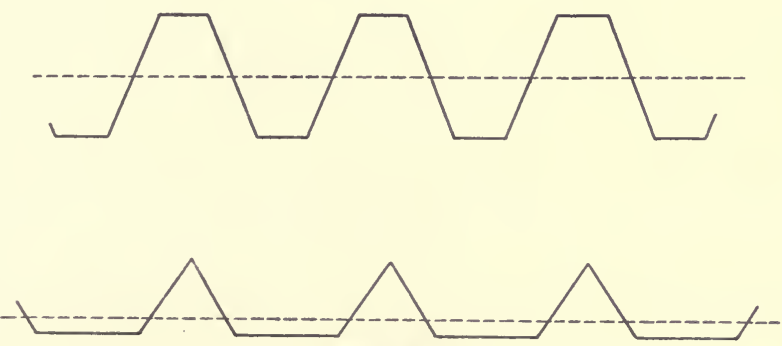

Fig. 28. 
In the latter case one of the intervals of rest vanishes*.

It is of course with the vibrations of a finite string that we are chiefly concerned in acoustics. The string is usually stretched with considerable tension between the two points which limit the vibrating portion. At one at least of these points the string passes over a bridge resting on a sounding-board, whose function it is to communicate the vibrations to the surrounding air. The direct action of the string in generating air-waves is quite insignificant, but by the alternating pressure on the bridge the whole area of the sounding-board is set into forced vibration. This implies of course a certain reaction on the string itself, which is however, in the first approximation, usually negligible, for the reason given in $\S 4$.

For experimental purposes an arrangement called a "monochord" is used. The sounding-board here forms the upper face of a rectangular "resonance chamber." The distance between the bridges can be varied and measured, and the tension, being produced by a weight attached to one end of the wire, which passes over a smooth pulley, can be regarded, at all events approximately, as known. For purposes of comparison one or more additional wires may be stretched alongside the former, their tension being adjusted, as in the pianoforte, by means of pegs at the extremities.

\section{Normal Modes of Finite String. Harmonics.}

The preceding investigations have been given on account of their historical importance, and for the sake of the analogies with other types of wave-motion which we shall meet with later. From the purely acoustical point of view they are however of secondary interest. The ear knows nothing of the particular geometrical forms assumed by the string, and is concerned solely with the frequencies and intensities of the simpleharmonic constituents into which the vibration can be resolved.

* The theoretical vibration-forms have been verified experimentally by Krigar-Menzel and Raps, Wied. Ann., vol. L., 1893, so far as the initial stages of the motion are concerned. After a few vibrations the form is seen to be undergoing a gradual change. This is attributed to a slight yielding of the - supports of the string, in consequence of which the normal frequencies are not exactly commensurable, and the resulting motion therefore not accurately periodic. The construction in Fig. 27 is also due to these writers. 
To ascertain the normal modes of vibration of a finite string we may have recourse to the general procedure explained in Chapter I. In any such mode $y$ will vary as a simple-harmonic function of the time, say $\cos (n t+\epsilon)$. This makes $\ddot{y}=-n^{2} y$, and the equation (2) of $\S 22$ therefore assumes the form

$$
\frac{\partial^{2} y}{\partial x^{2}}+\frac{n^{2}}{c^{2}} y=0 \text {. }
$$

The solution of this, exhibiting the time-factor, is

$$
y=\left(A \cos \frac{n x}{c}+B \sin \frac{n x}{c}\right) \cos (n t+\epsilon) .
$$

The fixed ends of the string being at $x=0, x=l$, we must have $A=0, \sin (n l / c)=0$, whence

$$
n l / \pi c=1,2,3, \ldots
$$

This gives the admissible values of $n$. In any one normal mode we have, therefore,

$$
y=C_{8} \sin \frac{s \pi x}{l} \cos \left(\frac{s \pi c t}{l}+\epsilon_{s}\right),
$$

where $s$ is an integer, and the amplitude $C_{s}$ and initial phase $\epsilon_{s}$ are arbitrary. The gravest, or fundamental mode, which determines the pitch of note sounded, corresponds to $s=1$.
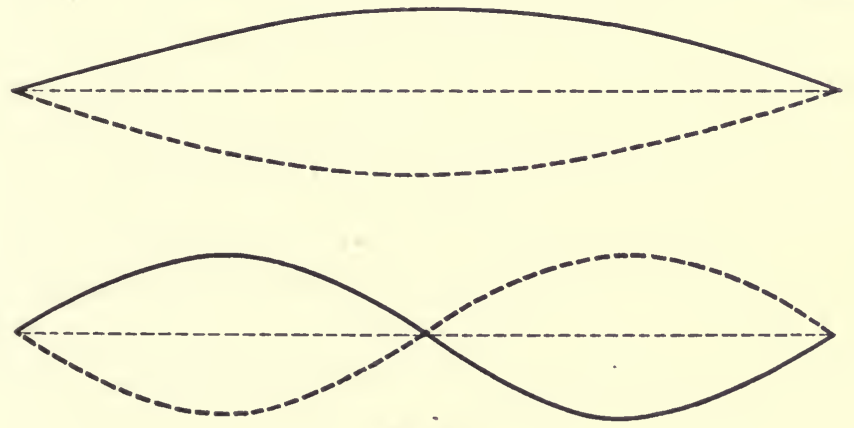

Fig. 29.

The string then oscillates in the form of the curve of sines between the two extreme positions shewn in the upper part of Fig. 29. The frequency is

$$
N=\frac{n}{2 \pi}=\frac{c}{2 l}=\frac{1}{2 l} \sqrt{ }\left(\frac{P}{\rho}\right),
$$


and so varies inversely as the length and as the square root of the line-density, and directly as the square root of the tension. These statements, which were formulated as the result of experiment long before the mathematical theory had been developed, are known as Mersenne's laws*. The determination of absolute pitch by the formula (5) does not admit of very great accuracy owing to the difficulty in measuring the tension, which is apt (owing to friction) to be slightly different on the two sides of a bridge.

The principles that the frequency diminishes with increase of length and with increase of line-density have a familiar illustration in the pianoforte, where longer and intrinsically heavier strings are used for the graver notes. If the relation of pitch were adjusted by length alone the strings corresponding to the lower notes would have to be at least 100 times as long as those belonging to the highest. In order to secure a sufficiently low pitch within practical limits of length, and with a sufficient degree of tension, the string is loaded with a coil of wire wrapped closely round it. This has the effect of increasing the inertia without seriously impairing the flexibility, which is an essential point. The influence of tension, again, is illustrated in the process of tuning, which consists in tightening up the wires when these have stretched, or the pegs have yielded, so that the instrument has fallen in pitch, or become "flat."

In the next normal mode after the fundamental the middle point $x=\frac{1}{2} l$ is at rest (Fig. 29). And in the, sth mode, whose frequency is by (3) $s$ times that of the fundamental, there are $s-1$ internal points of rest, or "nodes," in addition to the ends. Midway between these we have the points of maximum amplitude, or "loops." Each segment into which the string is divided by the nodes vibrates as in the fundamental mode of a string of $1 /$ sth the length.

As already stated $(\S 2)$ the sequence of simple vibrations with frequencies proportional to the natural numbers $1,2,3, \ldots$, which we here meet with, has important properties, musically

* M. Mersenne (1588-1648), a Franciscan friar, was a schoolfellow and lifelong friend of Descartes, and maintained an extensive correspondence with him and other men of science of the day. 
and physiologically. Its occurrence in vibrating systems is of course quite exceptional. Even in the present case, if the string deviate appreciably from uniformity or from perfect flexibility, the above scale of frequencies is at once departed from*.

We were led in $§ 16$ to the conclusion, on physical grounds, that in any system of finite extent the effect of the most general initial conditions consistent with its constitution may be obtained by superposition of the several normal modes, with suitable amplitudes and phase-constants. We infer that the most general motion of a finite string can be represented by the formula

$$
y=\Sigma C_{8} \sin \frac{s \pi x}{l} \cos \left(\frac{s \pi c t}{l}+\epsilon_{8}\right),
$$

provided the constants $C_{\boldsymbol{s}}, \epsilon_{\boldsymbol{8}}$ be properly determined, the summation $\Sigma$ extending over all integral values of $s$. An equivalent form is

$$
y=\Sigma\left(A_{8} \cos \frac{s \pi c t}{l}+B_{8} \sin \frac{s \pi c t}{l}\right) \sin \frac{s \pi x}{l},
$$

where

$$
A_{8}=C_{8} \cos \epsilon_{8}, \quad B_{8}=-C_{8} \sin \epsilon_{8} \text {. }
$$

If the string start from rest in a given position at the instant $t=0$ the coefficients $B_{s}$ will vanish; if it be started with given velocities from the equilibrium position $(y=0)$ the coefficients $A_{8}$ will vanish.

Since the value of every term in (6) or (7) recurs whenever $t$ is increased by $2 l / c$, the vibration is essentially periodic, as already proved in $\$ 24$. In all other respects the motion of the string when started in an arbitrary manner is, from the present point of view, of a complex character, being made up of an endless series of simple-harmonic vibrations. The resulting note is accordingly made up of a series of pure tones, consisting (in general) of a fundamental, its octave, twelfth, double octave, and so on.

It is not altogether easy to excite a string in such a way

* The fact that a particular sequence of notes, musically related to one another, is associated with lengths of string proportional to the quantities $1, \frac{1}{2}, \frac{1}{3}, \frac{1}{4}, \ldots$ was known to the Greeks, and was the origin of the name "harmonic" as applied to the numerical series. 
that the resulting motion shall be strictly simple-harmonic, and the sensation accordingly that of a pure tone. But, as will be shewn more fully in $\S 39$, it is possible to suppress all the tones below any assigned rank $(s)$ by checking the vibration at a node of the sth mode, as, for instance, by contact with a camel-hair pencil. The remaining nodal points of this constituent are then points of rest, whilst half-way between them there is vigorous vibration. The experiment, which is very striking, is easily made with the monochord.

The energy in any normal mode is easily calculated. We find

$$
\begin{aligned}
& T=\frac{1}{2} \rho \int_{0}^{l} \dot{y}^{2} d x=\frac{s^{2} \pi^{2} \rho c^{2}}{4 l} \cdot C_{8}{ }^{2} \sin ^{2}\left(\frac{s \pi c t}{l}+\epsilon_{8}\right), \\
& V=\frac{1}{2} P \int_{0}^{l} y^{\prime 2} d x=\frac{s^{2} \pi^{2} P}{4 l} \cdot C_{8}{ }^{2} \cos ^{2}\left(\frac{s \pi c t}{l}+\epsilon_{8}\right) .
\end{aligned}
$$

The coefficients are equal, in virtue of $\S 22(3)$, and the total energy in this mode is

$$
T+V=\frac{s^{2} \pi^{2} P}{4 l} \cdot C_{8}^{2} .
$$

It is further easily proved that the whole energy of the string is the sum of the energies corresponding to the various normal modes, viz.

$$
T+V=\frac{\pi^{2} P}{4 l} \sum s^{2} C_{s}{ }^{2}=\frac{\pi^{2} P}{4 l} \Sigma s^{2}\left(A_{s}{ }^{2}+B_{s}{ }^{2}\right) .
$$

This is a general property of the normal modes of a vibrating system. The proof, in the present case, depends on the fact that

$$
\int_{0}^{l} \sin \frac{s \pi x}{l} \sin \frac{s^{\prime} \pi x}{l} d x=0,
$$

if $s, s^{\prime}$ be any two unequal integers. See $§ 32(4)$.

\section{String excited by Plucking, or by Impact.}

The relative amplitudes of the various modes is of course a matter of importance, as on it the quality of the note depends (\$2). Usually a string is excited in one of three ways, viz. by plucking (as in the harp, zither, \&c.), by striking with a hammer (pianoforte), or by bowing (violin, violoncello, \&c.). 
If the string be pulled aside through a small space $\beta$, at a distance $a$ from the end $x=0$, and then be released, the values of the coefficients in $\$ 25(7)$ are found to be

$$
A_{s}=\frac{2 \beta l^{2}}{s^{2} \pi^{2} a(l-a)} \sin \frac{s \pi a}{l}, \quad B_{s}=0,
$$

whence $\quad y=\frac{2 \beta l^{2}}{\pi^{2} a(l-a)} \Sigma \frac{1}{s^{2}} \sin \frac{s \pi a}{l} \sin \frac{s \pi x}{l} \cos \frac{s \pi c t}{l}$.

The mode of calculation will be explained in the next chapter (see $\S 36$ ). We notice that the harmonic of order $s$ will be altogether absent if $\sin (s \pi a / l)=0$, i.e. if the point of plucking be at one of its nodes; this was remarked by Young (1841). Thus if the string be plucked at the centre, all the harmonics of even order will be absent. The formula (1) combined with $\S 25$ (12) shews that, apart from a trigonometrical factor which lies between 0 and 1 , the intensities of the successive harmonics will vary as $1 / s^{2}$. The higher harmonics are therefore relatively feebly represented in the actual vibration of the string.

The effect of the impact of a hammer depends on the manner and duration of the contact, and is more difficult to estimate. The question is indeed, strictly, one of forced vibrations $(\$ 28)$; but in the somewhat fictitious case where the duration is so small that the impact has ceased before the disturbance (travelling with the velocity $c$ ) has had time to spread over any appreciable fraction of the length, we may treat the problem as one of free motion with given initial velocity concentrated on a short length. The result is

$$
A_{8}=0, \quad B_{8}=\frac{2 \mu}{s \pi \rho c} \sin \frac{s \pi a}{l},
$$

where $a$ is the distance from the origin to the point struck, and $\mu$ represents the total momentum communicated by the impact. Hence

$$
y=\frac{2 \mu}{\pi \rho c} \Sigma \frac{1}{s} \sin \frac{s \pi a}{l} \sin \frac{s \pi x}{l} \sin \frac{s \pi c t}{l} .
$$

As in the previous problem, the sth mode is absent if the origin be at one of its nodes. Apart from the trigonometrical factor on which this circumstance depends, the intensities of 
the successive modes are, according to $\$ 25$ (12), now of the same order of magnitude. The unreal character of the preceding hypothesis betrays itself in this result; but we may at all events infer that in the case of a very brief impact the higher harmonics are relatively much more in evidence than in the former problem.

In reality the impact, even in the case of a metallic hammer, is far from instantaneous, the time of contact, though very short as measured by ordinary standards, being at all events comparable with the period of vibration of the string*. The effect of an impulse of finite duration has been calculated by Helmholtz, to whom most of the present theory is due, on the supposition that the pressure begins at the instant $t=0$, and lasts for a time $\tau$, during which it rises from zero to a maximum and falls to zero again, according to the law $\sin (\pi t / \tau)$. A somewhat simpler result is obtained if we imagine the law of pressure to be

$$
Y=\frac{2}{\pi} \frac{\mu \tau}{t^{2}+\tau^{2}}
$$

where $\mu$ represents the time-integral of the force from $t=-\infty$ to $t=+\infty$. This law, whose graphical representation has the form of the curve in Fig. 14, p. 33, has the defect that there is no definite instant of beginning or ending, but as the true law is in any case unknown, it may serve for purposes of illustration. The interval of time during which the force is sensible is comparable with $\tau$, and can be made as narrow as we please by diminishing $\tau$. The details of the calculation will more conveniently find a place in the next chapter $(\$ 38)$. The result is

$$
A_{s}=0, \quad B_{s}=\frac{2 \mu}{\pi \rho c} \cdot \frac{1}{s} e^{-s \pi c \tau / l} \sin \frac{s \pi a}{l} .
$$

When $\tau$ is infinitesimal this agrees with (3). In other cases the intensities of the higher harmonics vary as $e^{-2 s \pi c \tau / l}$, if we omit the trigonometrical factor.

Although the pressure is thus rendered less abrupt as regards its variation with the time, it is still assumed to be

* Kaufmann, Wied. Ann., vol. LIv. (1895). 
concentrated at a point. If we were to imagine it distributed continuously over a short length of the string this would further increase the relative weight of the lower harmonics (see § 38).

According to a general principle, which is here exemplified, and which will be further referred to in the next chapter, the higher harmonics are excited in greater relative intensity, the more abrupt the character of the originating disturbance. From a musical point of view the harmonics after about the sixth are to be discouraged, since they come sufficiently near to one another in the scale to be mutually discordant. In the pianoforte the hammers are covered with layers of softer material, so that the variation of pressure during the impact is rendered more gradual.

The point at which the blow is delivered is also a matter of importance. To obtain a note of rich musical quality the lower harmonics should be present in considerable force, and the middle regions of the string are on this account to be avoided. On the other hand, the harmonics of higher order than the sixth are prejudicial, as already stated. Both requirements are met by fixing the striking point at a distance of about one-seventh of the length from one end. The partial tones which have nodes at or near this point will then not be excited at all, or only with comparatively feeble intensity.

\section{Vibrations of a Violin String.}

The theory of the vibrations of a string when excited by bowing is somewhat difficult, but the main features have been elucidated by Helmholtz. Since the pitch is found to be that natural to the string, the vibrations are to be regarded as in a sense "free," the function of the bow being to maintain the motion by supplying energy to make up for the losses by dissipation. In the case of the violin \&c., where the strings are of light material and pass over a bridge resting on a very sensitive surface (of the resonance cavity), these losses may be relatively considerable. The mode of action of the bow appears to be that it drags the string with it for a time by friction, until at length the latter springs back; after a further 
interval the string is carried forward again, and so on*, the complete cycle taking place in the period of vibration.

In order to obtain data for mathematical analysis Helmholtz began by an experimental study of the character of the vibration at various points. The device was an optical one, of the kind employed by Lissajous ( $\$ 18$ ), by which the rectilinear vibration of the point examined is compounded with an independent vibration at right angles, whose period is commensurable, or nearly so, with that of the string. A microscope whose axis is horizontal is directed to the point to be studied, the string itself being vertical. The eye-piece of the microscope is fixed, but the objective is carried by one of the prongs of a tuning fork and vibrates in a vertical direction. When the fork alone vibrates the image of a bright point on the string is drawn out into a vertical line; when the string alone vibrates the appearance is that of a horizontal line. When both vibrations coexist the result would be a closed curve if the periods were exactly commensurable. For example, if the period of the fork were exactly commensurable with that of the string, and if the vibration of the point examined were simple-harmonic, the result would be one of the corresponding series of Lissajous figures $(\S 18)$; whilst if the relation between the periods were inexact, the curve would pass in succession through the various phases of the series. In the actual circumstances the forms of the curves are modified, and it is possible from the result to make inferences as to the true nature of the vibration studied.

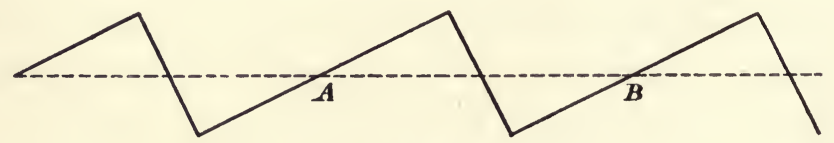

Fig. 30.

The interpretation is facilitated by the ideal representation of the successive phases as orthogonal projections of a curve traced on a revolving cylinder. It was found that the space-

* In order that work may be done it is necessary to suppose that the frictional force is greater in the first stage than in the second. This is consistent with the known law that friction of (relative) rest is greater than friction of motion. The remark is due to Lord Rayleigh. 
time diagram of the point of the string under examination has the simple form shewn in Fig. 30. The nature of the modification in the Lissajous figures may be illustrated in the case of unison between fork and string. If the portion of the broken line in Fig. 30 which lies between $A$ and $B$ be wrapped round a cylinder whose circumference is equal to $A B$, its projections on planes through the axis will include such forms as are here shewn (Fig. 31)*.

The period of vibration of the point examined is made up of two intervals, usually of unequal duration, during which the point moves backwards and forwards, respectively, with constant but (in general) unequal velocities. The ratio of the two intervals is further ascertained to be equal to that of the two segments into which the string is divided by the point. These results have been confirmed by subsequent observers who have obtained the space-time diagram in a more direct mannert. In order that they may come out clearly some precautions are necessary. Something depends on the skill with which the bow is used, and apparently on the quality of the instrument. In order, also, that the diagram should be free from minor irregularities the bow should be

* In the actual experiments of Helmholtz the frequency of the string was four times that of the fork. The circumference of the cylinder in the above mode of representation then includes four periods of the zig-zag line in Fig. 30.

+ Krigar-Menzel and Raps, Wied. Ann., vol. xLIV. (1891).
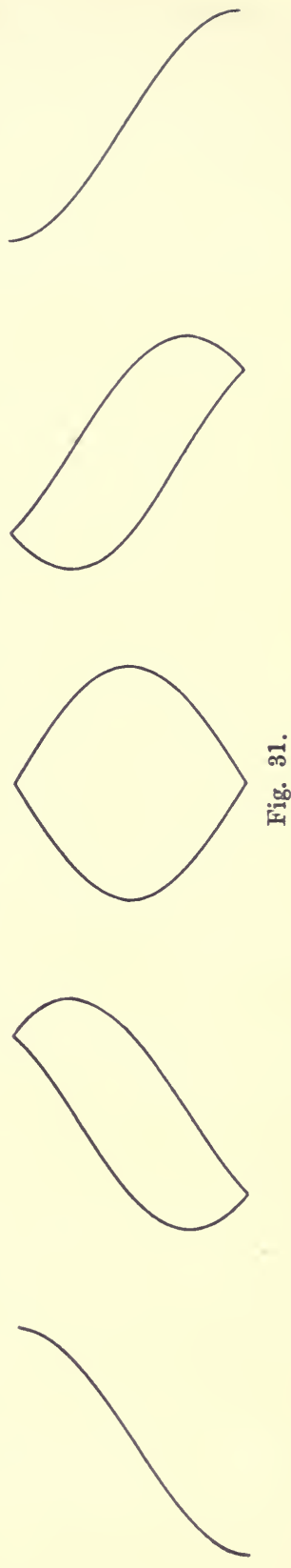
applied at a node of one of the harmonics, and the point observed should be at another node of the same.

Except at the two instants in each period when the velocity suddenly changes, the acceleration of the point $(P)$ examined is zero. It follows from $\$ 22$ (2) that the curvature of the string in the neighbourhood of $P$ vanishes, and that the form of the string at any instant is accordingly made up of straight pieces.

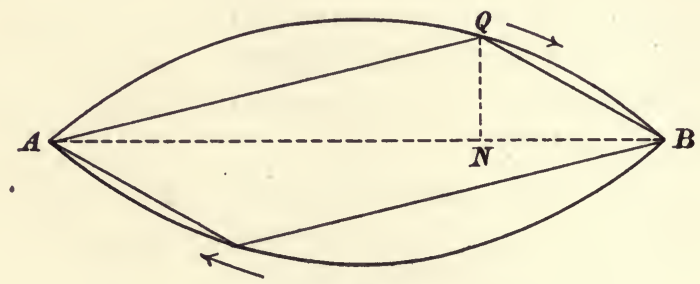

Fig. 32.

It appears that all the conditions of the problem can be satisfied if we assume that the form is always that of two such pieces meeting at a variable point $Q$. In Fig. 32 let $A B(=l)$ be the undisturbed position of the string, and let $\alpha(=A N)$ and $\beta$ $(=N Q)$ be the coordinates of $Q$ referred to $A$ as origin and $A B$ as axis of abscissae. The equations of the two portions of the string are

$$
y_{1}=\beta x / \alpha, \quad y_{2}=\beta(l-x) /(l-\alpha),
$$

and the difference of the velocities near $Q$ on the two sides is accordingly

$$
\dot{y}_{1}-\dot{y}_{2}=\frac{-l \beta \dot{\alpha}}{\alpha(l-\alpha)} .
$$

In the time $\delta t$ a length $\dot{a} \delta t$ of the string is traversed by the point $Q$, so that a mass $\rho \dot{\alpha} \delta t$ has its velocity increased by the above amount. This is the effect of the transverse force

$$
P y_{2}^{\prime}-P y_{1}^{\prime}=-\frac{P l \beta}{\alpha(l-\alpha)},
$$

where $P$ is the tension, acting for the time $\delta t$. Equating the change of momentum to the impulse of the force we find

$$
\dot{\alpha}^{2}=P / \rho=c^{2} \text {. }
$$


The point of discontinuity $Q$ (of the gradient) must therefore travel right or left with the velocity $c$.

Let us suppose that $Q$ starts from $A$ at the instant $t=0$, and that $\beta$ is at first positive. The observations of Helmholtz shew that the velocity at a point $x$, viz.

$$
\dot{y}_{2}=(l-x) \frac{d}{d t}\left(\frac{\beta}{l-\alpha}\right)=(l-x) c \frac{d}{d \alpha}\left(\frac{\beta}{l-\alpha}\right)
$$

is during an interval $x / c$ constant, whence

$$
\beta=C \alpha(l-\alpha),
$$

no additive constant being admissible, since $\beta$ must vanish with $\alpha$. This is the equation of a parabolic arc passing through $A, B$. The conditions of the problem are therefore all fulfilled if we imagine $Q$ to travel backwards and forwards along two such arcs, with velocity $c$, in the manner indicated in Fig. 32 . In terms of the maximum displacement $\beta_{0}$ we have $C= \pm 4 \beta_{0} / l^{2}$, and the equations of the two portions of the string at any instant are therefore

$$
y_{1}=\frac{4 \beta_{0}}{l^{2}}(l-\alpha) x, \quad y_{2}=\frac{4 \beta_{0}}{l^{2}} \alpha(l-x) .
$$

It only remains to resolve this motion into its simpleharmonic constituents. The details of the calculation are given in $\S 37$. The result is

$$
y=\frac{8 \beta_{0}}{\pi^{2}} \Sigma \frac{1}{s^{2}} \sin \frac{s \pi x}{l} \sin \frac{s \pi c t}{l},
$$

where the summation embraces all integral values of $s$. Comparing with $§ 25(7)$ we have

$$
A_{8}=0, \quad B_{8}=\frac{8 \beta_{0}}{\pi^{2} s^{2}} .
$$

These results, and indeed the whole investigation, take no account of the position of the point to which the bow is applied. It is plain, however, that the position of the bow must have some influence on the character of the vibration; and it is found in fact that those normal modes are absent which have a node at the point in question. It is for this reason that the somewhat idealized vibration-form which is adopted as a basis of calculation is only obtained in its purity at corresponding nodes. 


\section{Forced Vibrations of a String.}

The simplest case of forced vibration is where a given simple-harmonic motion

$$
y=\beta \cos (p t+\alpha)
$$

is imposed at a point $(x=a)$. The portions of the string on the two sides of this point are to be treated separately. The results are

$$
\left.\begin{array}{l}
y_{1}=\frac{\sin \frac{p x}{c}}{\sin \frac{p a}{c} \beta \cos (p t+\alpha) \quad[0<x<a],} \\
y_{2}=\frac{\sin \frac{p(l-x)}{c}}{\sin \frac{p(l-a)}{c}} \beta \cos (p t+\alpha) \quad[a<x<l],
\end{array}\right\}
$$

for these satisfy the general differential equation $\$ 22(2)$, they make $y_{1}=0$ for $x=0$, and $y_{2}=0$ for $x=l$, and they agree with (1) when $x=a$. The amplitude of $y_{1}$ or $y_{2}$ becomes very great, owing to the smallness of the denominator, whenever $p a / c$ or $p(l-a) / c$ is nearly equal to a multiple of $\pi$, i.e. when the imposed period $2 \pi / p$ approximates to a natural period of a string of length $a$ or $l-a$, respectively. To obtain a practical result in such cases we should have to take account of dissipative forces.

The case is illustrated by pressing the stem of a vibrating tuning fork on a piano string. The sound swells out powerfully whenever the portion of the string between the point of contact and either end has a natural mode in unison with the fork. This plan is recommended by Helmholtz as a means of producing pure tones, since the higher modes of the fork, not being harmonic with the fundamental, are not reinforced.

When a transverse force of amount $Y$ per unit length acts on the string, the equation (2) of $\S 22$ is replaced by

$$
\rho \frac{\partial^{2} y}{\partial t^{2}}=P \frac{\partial^{2} y}{\partial x^{2}}+Y \text {. }
$$

In general $Y$ will be a function both of $x$ and $t$.

The case of a periodic force $F \cos (p t+\alpha)$ concentrated on an infinitely short length of the wire at $x=a$ may be deduced from 
the formulae (2). The value of $\beta$ in terms of $F$ is found from the consideration that the force must just balance the pull of the string on this point, i.e.

$$
F \cos (p t+\alpha)=P y_{1}^{\prime}-P y_{2}^{\prime}
$$

for $x=a$. This leads to

$$
y_{1}=\frac{\sin \frac{p x}{c} \sin \frac{p(l-a)}{c}}{\frac{p}{c} \sin \frac{p l}{c}} \cdot \frac{F}{P} \cos (p t+\alpha) .
$$

The formula for $y_{2}$ differs only in that the letters $x$ and $a$ are interchanged; we have here an instance of the reciprocal theorem of $\S 17$, according to which the vibration at a point $x$ due to a periodic force at $a$ must be the same as the vibration at the point $a$ due to an equal force (of the same period) at $x$.

The amplitude becomes as a rule great when $\sin (p l / c)$ is small, i.e. when the imposed period approaches a natural period of the whole string. An indeterminate case occurs when $\sin (p a / c)=0$ and $\sin (p l / c)=0$ simultaneously, the point $x=a$ being then a node.

\section{Qualifications to the Theory of Strings.}

We have in $\$ 26,27$ considered the relative amplitudes of the different harmonics when a string is excited in various ways, but we must not assume that the corresponding relative intensities are accurately reproduced in the resulting sound-waves, which are started indirectly through the sounding board. If we neglect the reaction on the string, which may for a considerable number of vibrations be insensible, we may regard the string as exerting on each bridge a force proportional to the value of $\partial y / \partial x$ there*, as given by the respective formula. The differentiation introduces a factor $s$ in the coefficient of the sth harmonic, and so increases the importance of the higher modes. On the other hand, the amplitude of vibration of the sounding board due to a simple-harmonic force of given amplitude, will vary somewhat with the frequency,

* Thus in the case of the plucked string it appears from Fig. 27 that the pressure on each end alternates between two constant values of opposite sign. 
on the general principle illustrated in $\S 9^{*}$. This is probably to the relative advantage of the lower modes. The effect of yielding of the bridges in modifying the natural frequencies of the string has been discussed by Rayleigh + ; it is probably in practice very slight.

Another cause which must be mentioned as affecting our results to some extent is the imperfect flexibility of the string, or wire. In the case of the higher normal modes the segments into which the string is divided may be so short that flexural couples come into play, and tend to raise the frequency by increasing the potential energy of a given deformation. This will be referred to later $(\$ 50)$. A further point is that the abrupt forms postulated in the theory of plucked or bowed strings are not exactly realized, and that such investigations as those of $\$ \S 26,27$ are to be viewed as approximations, which are however quite adequate so far as the determination of the amplitudes of the graver and more important harmonics is concerned.

\section{Vibrations of a Loaded String.}

We conclude this chapter with the discussion of one or two problems which, besides being of some interest in themselves, may serve to remind us again that the harmonic scale of frequencies is after all an exceptional phenomenon, even in the case of strings.

Take first the case of a string, otherwise uniform, loaded with a mass $M$ at its centre. It is obvious that those normal modes of the unloaded string which have a node at this point are unaffected. Leaving these on one side, we consider only those vibrations in which there is at every instant complete symmetry with regard to the centre. If the lateral displacement of $M$ be $\beta \cos (n t+\epsilon)$, we have, for the first half of the string,

$$
y_{1}=\frac{\sin \frac{n x}{c}}{\sin \frac{n l}{2 c}} \beta \cos (n t+\epsilon) .
$$

* Some interesting experiments bearing on these questions have been made by Barton and Garrett, Phil. Mag. (6), vol. x., 1905. See also Barton, TextBook of Sound, London, 1908, § 361 .

+ Theory of Sound, § 135. 
The equation of motion of $M$ is

$$
M \ddot{y}_{1}=-2 P y_{1}^{\prime},
$$

where after the differentiations we must suppose $x=\frac{1}{2} l$. This gives

$$
\frac{n l}{2 c} \tan \frac{n l}{2 c}=\frac{l}{b},
$$

where $b$ is written for $M / \rho$, i.e. $b$ is the length of string whose mass would be equal to that of the attached particle. The frequencies are therefore determined by

$$
n l / 2 c=x_{1}, x_{2}, x_{3}, \ldots,
$$

where $x_{1}, x_{2}, x_{3}, \ldots$ are the roots of the transcendental equation

$$
x \tan x=l / b \text {. }
$$

Equations more or less of this character occur in many branches of mathematical physics, and can often be solved approximately by graphical construction. Thus in the present instance if we trace the curves

$$
y=\cot x, \quad y=\frac{b}{l} \cdot x,
$$

the abscissae give the roots. If $b$ be relatively small these fall a little short of $\frac{1}{2} \pi, \frac{3}{2} \pi, \frac{5}{2} \pi, \ldots$, respectively, and the

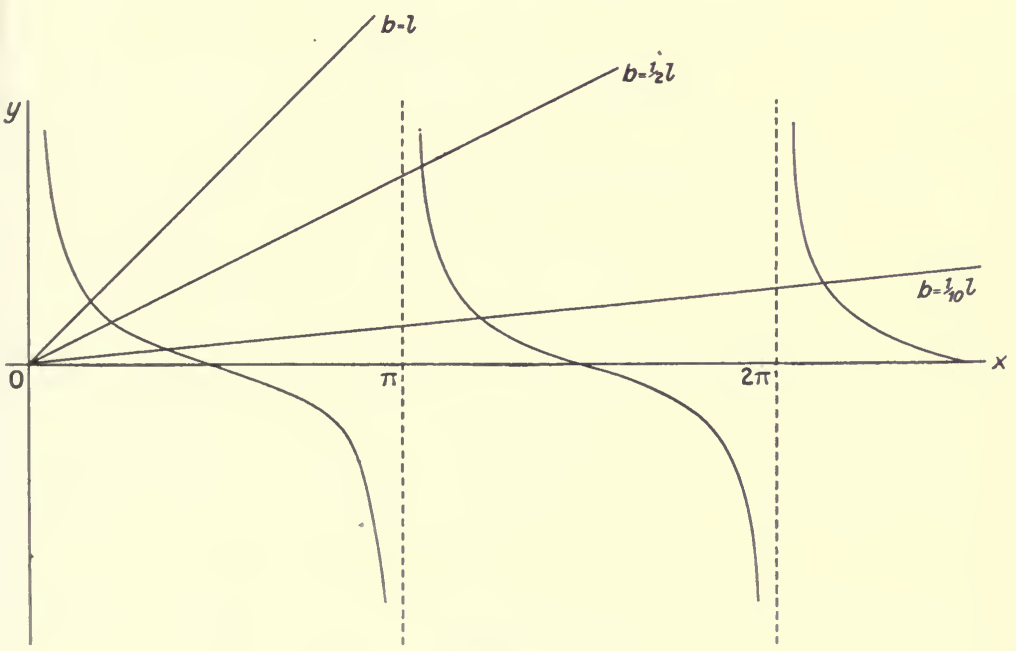

Fig. 33. 
frequencies are therefore slightly lower than in the symmetric modes of an unloaded string. As $b$ increases the frequencies all diminish, the physical reason being of course the increased inertia. Finally, when $M$ is very large compared with the mass $\rho l$ of the string, $l / b$ is small, and the lowest root of $(5)$ is given approximately by $x^{2}=l / b$, whence

$$
n=\frac{2 c}{\sqrt{ }(l b)}=2 \sqrt{ }\left(\frac{P}{M l}\right)
$$

in agreement with $\S 6(4)$.

\section{Hanging Chain.}

The contrast with previous continuous systems is still more marked in the case of the small oscillations of a uniform chain hanging vertically from its upper end, which is fixed. This has no immediate acoustic importance, but it is interesting historically*, and is, from the standpoint of general theory, instructive in various ways.

We take the origin at the equilibrium position of the free end. The tension at a height $x$ above this point will be $P=g \rho x$, the vertical motion being neglected as of the second order. Hence if $y$ denote the horizontal deflection, we have

$$
\begin{aligned}
& \rho \frac{\partial^{2} y}{\partial t^{2}}=\frac{\partial}{\partial x}\left(P \frac{\partial y}{\partial x}\right), \\
& \frac{\partial^{2} y}{\partial t^{2}}=g \frac{\partial}{\partial x}\left(x \frac{\partial y}{\partial x}\right) .
\end{aligned}
$$

To ascertain the normal modes we assume that $y$ varies as $\cos (n t+\epsilon)$, and obtain

$$
\frac{\partial}{\partial x}\left(x \frac{\partial y}{\partial x}\right)+\frac{n^{2}}{g} y=0
$$

This can be integrated by a series, but the solution assumes a somewhat neater form if we introduce a new independent variable in place of $x$. The wave-velocity on a string having a uniform tension equal to that which obtains at the point $x$ would be $\sqrt{ }(P / \rho)$ or $\sqrt{ }(g x)$. Hence if $\tau$ denotes the time which

* It appears to have been the first instance in which the various normal modes of a continuous system were determined, viz. by D. Bernoulli (1732). The Bessel's Function also makes its first appearance in this connection. 
a point moving always with this local wave-velocity would take to travel from the lower end to the point $x$, we have

$$
\tau=\int_{0}^{x} \frac{d x}{\sqrt{ }(g x)}=2 \sqrt{\left(\frac{x}{g}\right), \quad x=\frac{1}{4} g \tau^{2} .}
$$

In terms of $\tau$ as independent variable the equation (3) becomes

$$
\frac{\partial^{2} y}{\partial \tau^{2}}+\frac{1}{\tau} \frac{\partial y}{\partial \tau}+n^{2} y=0
$$

For the present purpose we do not require the complete solution, but only that solution which remains finite when $\tau=0$. This is

$$
y=C\left(1-\frac{n^{2} \tau^{2}}{2^{2}}+\frac{n^{4} \tau^{4}}{2^{2} .4^{2}}-\ldots\right)
$$

where $C$ is arbitrary, as may be verified by actual differentiation, and substitution in (5). The function defined by the series in brackets presents itself in many physical problems; it is called the "Bessel's Function of Zero Order," and is denoted by $J_{0}(n \tau)^{*}$. Hence, inserting the time-factor,

$$
y=C J_{0}(n \tau) \cos (n t+\epsilon) \text {. }
$$

The value of $\tau$ corresponding to the upper end $(x=l)$ is

$$
\tau_{1}=2 \sqrt{ }(l / g)
$$

and the condition that this end should be fixed gives

$$
J_{0}\left(n \tau_{1}\right)=0 \text {. }
$$

This determines the admissible values of $n$. The first few roots are given by

$$
n \tau_{1} / \pi=\cdot 7655,1 \cdot 7571,2 \cdot 7546, \ldots,
$$

where the numbers tend to the form $s-\frac{1}{4}, s$ being integral. In the modes after the first, the values of $\tau$ corresponding to the lower roots give the nodes. Thus in the second mode there is a node at the point $\tau / \tau_{1}=\cdot 7655 / 1 \cdot 7571$, or $x / l=\tau^{2} / \tau_{1}{ }^{2}=\cdot 190$. The gravest period is $2 \pi / n=5 \cdot 225 \mathrm{~V}(l / g)$, whereas the period of oscillation of a rigid bar of the same length is $5 \cdot 130 \sqrt{ }(l / g)$. The comparison verifies a general principle referred to in $\S 16$,

* Elaborate numerical tables of the Bessel's Functions, calculated by Meissel and others, are given by Gray and Mathews, Treatise on Bessel Functions, London, 1895. A convenient abridgment is included in Dale's Five-Figure Tables of Mathematical Functions, London, 1903. 
according to which any constraint has the effect of quickening the gravest oscillation. The first two modes are shewn (on different scales) in Fig. $3 t$, the two nodal points representing the point of suspension in the two cases.

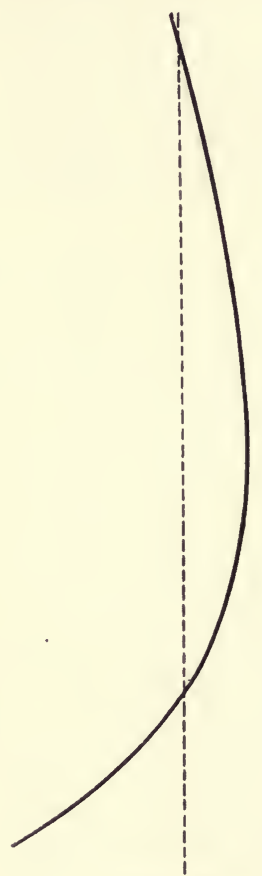

Fig. 34 . 


\section{CHAPTER III}

\section{FOURIER'S THEOREM}

\section{The Sine-Series.}

The study of the transverse vibrations of strings has already suggested a remarkable theorem of pure mathematics, to which some further attention must now be given. The theory of the normal modes has led us $(\$ 25)$ to the conclusion that the free motion of a string of length $l$, started in any arbitrary manner, can be expressed by a series of the form

$$
y=\Sigma\left(A_{8} \cos \frac{s \pi c t}{l}+B_{8} \sin \frac{s \pi c t}{l}\right) \sin \frac{s \pi x}{l},
$$

where $s=1,2,3, \ldots$, provided the constants $A_{8}, B_{8}$ be properly determined. In particular if the string be supposed to start from rest at the instant $t=0$ in the arbitrary form $y=f(x)$, it should be possible to determine the coefficients $A_{g}$ so that

$$
f(x)=\Sigma A_{8} \sin \frac{s \pi x}{l},
$$

for values of $x$ ranging from $x=0$ to $x=l$. This is a particular case of "Fourier's Theorem*." Since $l$ is at our disposal we may conveniently replace it (for general purposes) by $\pi$, and the statement then is that an arbitrary function $f(x)$ can be expressed, for values of $x$ ranging from 0 to $\pi$, in the form

$$
f(x)=A_{1} \sin x+A_{2} \sin 2 x+\ldots+A_{8} \sin s x+\ldots
$$

* J. B. J. Fourier (1768-1830). The history of the theorem is closely interwoven with that of the theory of strings, and of the theory of heatconduction. Fourier's own researches are expounded in his Théorie de la Chaleur, Paris, 1822. An ontline of the history is given in Prof. Carslaw's book cited on p. 96. The subject is treated most fully by H. Burkhardt in his report entitled Entwickelungen nach oscillirenden Funktionen..., Leipzig, 1908. 
The reasoning by which we have been led to this result is of a physical rather than a mathematical nature, and we have moreover not referred to the restrictions which physical considerations alone would impose on the character of the arbitrary function $f(x)$. Leaving such points for the moment, and assuming the theorem provisionally, we proceed to the determination of the coefficients. If we multiply both sides of (3) by $\sin s x$, and integrate from $x=0$ to $x=\pi$, we get on the right hand a series whose general term is

$A_{r} \int_{0}^{\pi} \sin r x \sin s x d x$

$$
=\frac{1}{2} A_{r} \int_{0}^{\pi}\{\cos (r-s) x-\cos (r+s) x\} d x .
$$

When the integers $r, s$ are unequal this vanishes, since each cosine goes through its cycle of values, positive and negative, once or oftener within the range of integration. But when $r=s$, the first cosine is replaced by unity, and the result is $\frac{1}{2} \pi A_{8}$. Hence

$$
A_{s}=\frac{2}{\pi} \int_{0}^{\pi} f(x) \sin s x d x
$$

The process may be illustrated by a few examples. Take, first, the case of

$$
f(x)=x(\pi-x)
$$

which is represented by an arc of a parabola. We find, after a series of partial integrations,

$$
A_{8}=\frac{2}{\pi} \int_{0}^{\pi} x(\pi-x) \sin s x d x=\frac{4}{\pi s^{3}}(1-\cos s \pi) .
$$

This is equal to 0 or $8 / \pi s^{3}$, according as $s$ is even or odd. The theorem therefore becomes

$$
x(\pi-x)=\frac{8}{\pi}\left(\sin x+\frac{1}{3^{3}} \sin 3 x+\frac{1}{5^{3}} \sin 5 x+\ldots\right) .
$$

If we put $x=\frac{1}{2} \pi$ in this we obtain the formula

$$
\frac{\pi^{3}}{32}=1-\frac{1}{3^{3}}+\frac{1}{5^{3}}-\ldots,
$$

which is known on other grounds to be correct. The equality in (8) may also be tested graphically. It is found that the discrepancy between the graph of $x(\pi-x)$ and that of the 
function represented by the first three terms on the right hand is so slight that it would be barely perceptible on a scale suited to the pages of this book.

In the next example the graph of $f(x)$ consists of two straight lines through the points $x=0, x=\pi$, respectively, meeting at an angle at the point $x=\alpha$. If we assume the ordinate at the latter point to be unity, we have

$$
\left.\begin{array}{ll}
f(x)=x / \alpha & {[0<x<\alpha],} \\
f(x)=(\pi-x) /(\pi-\alpha) & {[\alpha<x<\pi] .}
\end{array}\right\}
$$

We find, after some reductions,

$$
\begin{array}{r}
A_{8}=\frac{2}{\pi \alpha} \int_{0}^{\alpha} x \sin s x d x+\frac{2}{\pi(\pi-\alpha)} \int_{\alpha}^{\pi}(\pi-x) \sin s x d x \\
=\frac{2}{\alpha(\pi-\alpha)} \cdot \frac{1}{s^{2}} \sin s \alpha .
\end{array}
$$

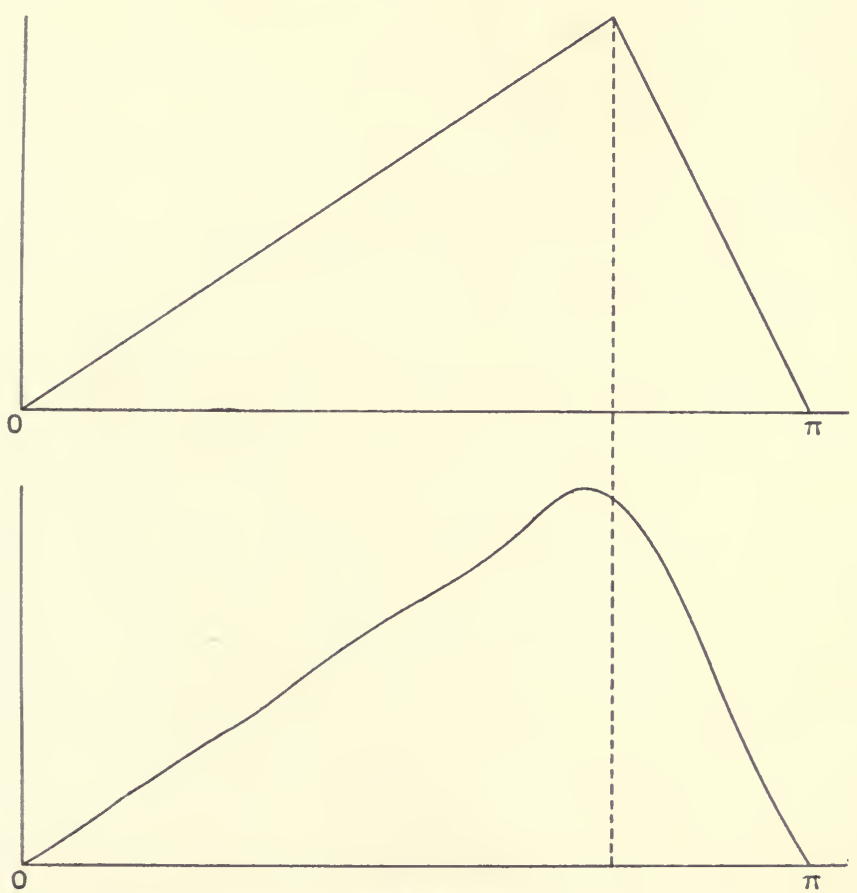

Fig. 35 . 
Thus

$$
\begin{aligned}
f(x)=\frac{2}{\alpha(\pi-\alpha)}(\sin \alpha \sin x & +\frac{1}{2^{2}} \sin 2 \alpha \sin 2 x \\
& \left.+\frac{1}{3^{2}} \sin 3 \alpha \sin 3 x+\ldots\right) .
\end{aligned}
$$

As a check on this result we may put $\alpha=\frac{1}{2} \pi, x=\frac{1}{2} \pi$; this gives

$$
\frac{\pi^{2}}{8}=1+\frac{1}{3^{2}}+\frac{1}{5^{2}}+\ldots
$$

which is known to be right. This example is of interest in connection with the theory of the plucked string (\$26,36). Fig. 35 shews the graph of $f(x)$ together with that of the function represented by the first eight terms of the series on the right hand of equation (12), in the case of $\alpha=\frac{3}{4} \pi$. The fourth and eighth terms contribute nothing to the result in this case, since they correspond to modes having a node at the point plucked.

Again, let $\quad f(x)=\pi-x$.

We find

$$
A_{8}=\frac{2}{\pi} \int_{0}^{\pi}(\pi-x) \sin s x d x=\frac{2}{s} .
$$

The theorem therefore asserts that

$$
\pi-x=2\left(\sin x+\frac{1}{2} \sin 2 x+\frac{1}{3} \sin 3 x+\ldots\right) .
$$

If we put $x=\frac{1}{2} \pi$, we obtain

$$
\frac{\pi}{4}=1-\frac{1}{3}+\frac{1}{5}-\ldots
$$

which is Euler's formula for the quadrature of the circle. The formula (16) also verifies obviously for $x=\pi$; but if we put $x=0$ we see that there is some limitation to its validity. The necessary modification is stated in $\S 34$. The series is moreover much more slowly convergent than in the preceding case; this is illustrated by Fig. 36, which shews the graph of $\pi-x$ together with that of the function represented by the first eight terms of the series. For any value of $x$ other than 0 we can obtain an approximation as close as we please, provided we take a sufficient number of terms, but the smaller the value of $x$ the greater will be the number of terms required to attain a prescribed standard. 


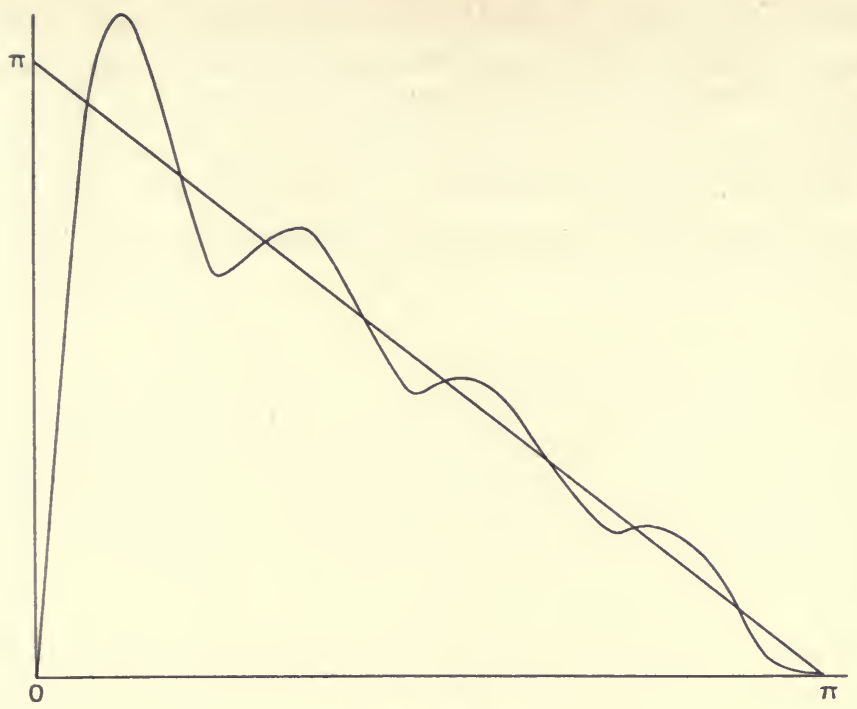

Fig. 36 .

The preceding illustrations, with the diagrams, afford at all events a presumption in favour of the theorem in question, but shew at the same time that it is subject to some restrictions. The theorem admits of independent mathematical proof under certain conditions as to the nature of the "arbitrary" function $f(x)$. We shall, however, not enter upon this, but shall content ourselves with the following formal statement:

If we form the sum of the first $m$ terms of the series (2), and write

$$
f_{m}(x)=A_{1} \sin x+A_{2} \sin 2 x+\ldots+A_{m} \sin m x,
$$

where

$$
A_{s}=\frac{2}{\pi} \int_{0}^{\pi} f(x) \sin s x d x,
$$

it may be shewn that, for any assigned value of $x$ in the range from 0 to $\pi$, the sum $f_{m}(x)$ will tend with increasing $m$ to the limit $f(x)$, provided the function $f(x)$ is continuous throughout the above range, has only a finite number of maxima and minima, and vanishes for $x=0$ and $x=\pi$.

It will be noticed that the conditions here postulated are 
fulfilled as a matter of course by any function which it is natural to assume as representing the initial form, or the initial velocity, of a tense string. We also see that the difficulty met with in the case of (16) can be accounted for by the fact that the function does not vanish for $x=0$. An extension of the statement to meet such cases will be given presently $(\S 34)$.

\section{The Cosine-Series.}

The theory of the longitudinal vibrations of rods, or of columns of air, leads, in addition, to a similar theorem relating to the expansion of an arbitrary function in a series of cosines. The formal statement is now as follows:

If we write

$$
f_{m}(x)=A_{0}+A_{1} \cos x+A_{2} \cos 2 x+\ldots+A_{m} \cos m x,
$$

where

$$
A_{0}=\frac{1}{\pi} \int_{0}^{\pi} f(x) d x
$$

whilst for $s>0$

$$
A_{8}=\frac{2}{\pi} \int_{0}^{\pi} f(x) \cos s x d x,
$$

it may be shewn that as $m$ increases the sum $f_{m}(x)$ will tend to the limit $f(x)$, provided $f(x)$ is continuous throughout the range from 0 to $\pi$, and has at most a finite number of maxima and minima. There is now no restriction as to the values of $f(0)$ and $f(\pi)$.

If the determination of the effect of special initial conditions in a longitudinally vibrating bar which is free at both ends were as interesting a problem as it is in the case of strings we should have recourse to the cosine-series.

\section{Complete Form of Fourier's Theorem. Discon-} tinuities.

The question arises as to what is represented by the sine-series or the cosine-series, supposed continued to infinity, when $x$ lies outside the limits 0 and $\pi$. The answer is supplied by the consideration that both series are periodic functions of $x$, the period being $2 \pi$, whilst the former is an odd, the 
whilst for $s>0$

$$
\left.\begin{array}{l}
A_{s}=\frac{1}{\pi} \int_{0}^{\pi}\{f(x)+f(-x)\} \cos s x d x=\frac{1}{\pi} \int_{-\pi}^{\pi} f(x) \cos s x d x, \\
B_{8}=\frac{1}{\pi} \int_{0}^{\pi}\{f(x)-f(-x)\} \sin s x d x=\frac{1}{\pi} \int_{-\pi}^{\pi} f(x) \sin s x d x,
\end{array}\right\}
$$

tends with increasing $m$ to the limit $f(x)$, provided $f(x)$ is continuous from $x=-\pi$ to $x=\pi$ and has at most a finite number of maxima and minima, and provided also that $f(-\pi)=f(\pi)$. For values of $x$ outside this range the limit represents, under these conditions, a periodic function of period $2 \pi$. This is the complete form of Fourier's Theorem, and includes the others as special cases.

We should be led directly, on physical grounds, to this form of the theorem if we were to investigate the "longitudinal" vibrations of the column of air in a reentrant circular tube.

We have so far supposed the function $f(x)$ to be continuous, as well as finite, even when continued beyond the original range as a periodic function. But the theorems hold, with a modification to be stated immediately, even if $f(x)$ have a finite number of isolated discontinuities. In such a case the series $f_{m}(x)$ still converges, with increasing $m$, to the value of $f(x)$, except at the points of discontinuity. But if $a$ be a point where $f(x)$ abruptly changes its value, the sum $f_{m}(a)$ tends to the limit

$$
\frac{1}{2}\{. f(a-0)+f(a+0)\}
$$

where $f(a-0)$ and $f(a+0)$ represent the values of $f(x)$ at infinitesimal distances to the left and right, respectively, of the point $a$. For example, in the case of the sine-series $\$ 32(3)$, if $f(x)$ does not vanish when $x=0$ or when $x=\pi$, there is discontinuity at these points in the periodic function, and the series $f_{m}(0)$, for example, has the limit 0 , which is the arithmetic mean of the values of the continued function on the two sides of the point $x=0$. This is illustrated in Fig. 36.

\section{Law of Convergence of Coefficients.}

It remains to say something as to the law of decrease of the successive terms. It is evident at once that under the 
conditions laid down the values of the coefficients $A_{8}$ and $B_{8}$ must ultimately diminish indefinitely as $s$ increases, owing to the more and more rapid fluctuation in sign of $\cos s x$ and $\sin s x$, and the consequent more complete cancelling of the various elements in the definite integrals of $\S 32(5)$ and $\S 33$ (3).

More definite results have been formulated by Stokes. The following statement must be understood to refer to the function as continued in the manner above explained; and care is necessary, in particular cases, to see whether discontinuities of $f(x)$ or its derivatives are introduced at the terminal points of the various segments:

If $f(x)$ have (in a period) a finite number of isolated discontinuities, the coefficients converge ultimately towards zero like the members of the sequence

$$
1, \frac{1}{2}, \frac{1}{3}, \frac{1}{4}, \ldots
$$

This is exemplified by $\S 32(16)$ and Fig. 36.

If $f(x)$ is everywhere continuous, whilst its first derivative $f^{\prime}(x)$ has a finite number of isolated discontinuities, the convergence is ultimately that of the sequence

$$
1, \frac{1}{2^{2}}, \frac{1}{3^{2}}, \frac{1}{4^{2}}, \ldots
$$

This is illustrated by $\S 32(12)$ and Fig. 35.

If $f(x), f^{\prime}(x)$ are continuous, whilst $f^{\prime \prime}(x)$ is discontinuous at isolated points, the sequence of comparison is

$$
1, \frac{1}{2^{3}}, \frac{1}{3^{3}}, \frac{1}{4^{3}}, \ldots
$$

as in the case of $\S 32$ (8). And, generally, if $f(x)$ and its derivatives up to the order $n-1$ inclusive are continuous, whilst the $n$th derivative has (in a period) a finite number of isolated discontinuities, the convergency is ultimately as

$$
1, \frac{1}{2^{n+1}}, \frac{1}{3^{n+1}}, \frac{1}{4^{n+1}}, \ldots
$$

The nature of the proof, which is simple, may be briefly 
indicated for the case of the sine-series. We have, by a partial integration,

$$
\begin{aligned}
A_{s} & =\frac{2}{\pi} \int_{0}^{\pi} f(x) \sin s x d x \\
& =-\frac{1}{s}\left[\frac{2}{\pi} f(x) \cos s x\right]+\frac{2}{s \pi} \int_{0}^{\pi} f^{\prime}(x) \cos s x d x,
\end{aligned}
$$

where the integrated term is to be calculated separately for each of the segments lying between the points of discontinuity of $f(x)$, if any, which occur in the range extending from $x=0$ to $x=\pi$ inclusively. For example, if as in $\S 32$ (14) the only discontinuity is at $x=0$, its value is $2 f(0) / s \pi$. In any case there is, for all values of $s$, an upper limit to the coefficient of $1 / s$ in the first part of (4); we denote this limit by $M$. The definite integral in the second term tends ultimately to zero, as $s$ increases, owing to the fluctuations in sign of $\cos s x$. Hence $A_{8}$ is ultimately comparable with $M / s$. If there is no discontinuity of $f(x)$, even at the points $x=0, x=\pi$, the first term in the above value of $A_{s}$ vanishes, and continuing the integration we find

$$
A_{8}=\frac{1}{s^{2}}\left[\frac{2}{\pi} f^{\prime}(x) \sin s x\right]-\frac{2}{s^{2} \pi} \int_{0}^{\pi} f^{\prime \prime}(x) \sin s x d x .
$$

In the first part, regard must be had to the discontinuities of $f^{\prime}(x)$, if any. Denoting by $M$ the upper limit of the coefficient of $1 / s^{2}$, we see that $A_{s}$ is ultimately comparable with $M / s^{2}$, the second term in (5) vanishing in comparison, by the principle of fluctuation. The further course of the argument is now sufficiently apparent.

\section{Physical Approximation. Case of Plucked String.}

It has been thought worth while to state Fourier's theorem with some care, although we do not enter into the details of the mathematical proof, which is necessarily somewhat intricate, owing to the various restrictions which are involved*.

From a physical point of view the matter may be dealt with, and perhaps adequately, in a much simpler manner. To explain this, it is best to take a definite problem, for instance that of

* The most recent English treatise on the subject is that of Prof. H. S. Carslaw, Fourier's Series and Integrals, London, 1906. 
the plucked string $(\$ 26)$. The differential equation, and the terminal conditions, are satisfied by the finite series

$$
\begin{aligned}
y=A_{1} \sin \frac{\pi x}{l} \cos \frac{\pi c t}{l}+ & A_{2} \sin \frac{2 \pi x}{l} \cos \frac{2 \pi c t}{l}+\ldots \\
& +A_{m} \sin \frac{m \pi x}{l} \cos \frac{m \pi c t}{l},
\end{aligned}
$$

each term of which represents a normal mode of vibration. This makes the initial velocity zero, whilst the initial form is

$$
y=A_{1} \sin \frac{\pi x}{l}+A_{2} \sin \frac{2 \pi x}{l}+\ldots+A_{m} \sin \frac{m \pi x}{l} .
$$

The question we now have to consider is, how to determine the coefficients $A_{1}, A_{2}, \ldots A_{n}$ so that (2) may represent, as closely as may be, a prescribed initial form

$$
y=f(x) \text {. }
$$

There are many reasons why, from the physical point of view, we may be content with an approximate solution of the problem. Leaving aside such questions as the resistance of the air and the yielding of the supports at the ends of the string, we have still to remember that in substituting a mathematical line of matter, capable only of exerting tension, we have considerably over-idealized the circumstances. In the higher normal modes, at all events, the imperfect flexibility, and the uncertainty as to the true nature of the terminal conditions, render this representation somewhat inadequate, so that a solution which professes to determine these modes accurately is open to the criticism that it attempts too much. Again, the assumed initial form in which two straight pieces meet at a point, is one which can only be approximately realized; if we go too far in this direction we should produce a permanent bend, or kink, in an actual wire.

The determination of the coefficients in the finite series (2) will depend on the kind of approximation aimed at. For example, we might divide the length of the string into $m+1$ equal parts, and choose the coefficients'so that the functions (2) and (3) should be equal at the $m$ dividing points. The curves represented by these equations will then intersect in $m$ points in addition to the ends. Another method is to make the sum of 
the squares of the errors involved in the substitution of (2) for (3) as small as possible. Thus if, for shortness, we replace $l$ by $\pi$, we have to choose the coefficients so as to make the integral

$$
\int_{0}^{\pi}\left\{f(x)-\left(A_{1} \sin x+A_{2} \sin 2 x+\ldots+A_{m} \sin m x\right)\right\}^{2} d x
$$

a minimum. If we differentiate with respect to $A_{s}$ we get

$$
\begin{aligned}
& \int_{0}^{\pi}\left\{f(x)-\left(A_{1} \sin x+A_{2} \sin 2 x+\ldots+A_{m} \sin m x\right)\right\} \sin s x d x=0, \\
& \text { or, by } \S 32(4), \\
& \qquad A_{8}=\frac{2}{\pi} \int_{0}^{\pi} f(x) \sin s x d x . \quad \ldots \ldots \ldots \ldots . . .
\end{aligned}
$$

Hence this method of least squares, applied to the expression (2) consisting of a finite number of terms, gives precisely the values of the coefficients which were obtained by Fourier's process*. Each coefficient is determined by itself, and the effect of adding more terms to (2) is to improve the approximation, without affecting the values of the coefficients already found. If we revert to general units, the formula (6) is replaced by

$$
A_{8}=\frac{2}{l} \int_{0}^{l} f(x) \sin \frac{s \pi x}{l} d x .
$$

In the case of the plucked string, the form to which we endeavour to approximate is

$$
y=\beta x / a \quad[0<x<a], \quad y=\beta(l-x) /(l-a) \quad[a<x<l] .
$$

The result is obtained at once from $\S 32$ (11) if we write $\pi x / l$ for $x$, and therefore $\pi a / l$ for $\alpha, l$ for $\pi$, and introduce the factor $\beta$. Thus

$$
A_{8}=\frac{2 \beta l^{2}}{s^{2} \pi^{2} a(l-a)} \sin \frac{s \pi a}{l},
$$

as stated in $\S 26$ (1). The nature of the approximation is illustrated in Fig. 35.

\section{Application to Violin String.}

To apply the method to the problem of the violin string ( $(27)$, we take as origin of $t$ the instant when the point $Q$ in

* This theorem is due to A. Toepler (1876). 
Fig. 32 starts from $A$ to describe the upper parabolic arc. At this instant we have $y=0$, everywhere, whilst

$$
\dot{y}=\frac{4 \beta_{0} c}{l^{2}}(l-x) \quad[t=0] .
$$

We therefore begin with the finite series

$$
\begin{aligned}
y=A_{1} \sin \frac{\pi x}{l} \sin \frac{\pi c t}{l}+ & A_{2} \sin \frac{2 \pi x}{l} \sin \frac{2 \pi c t}{l}+\ldots \\
& +A_{m} \sin \frac{m \pi x}{l} \sin \frac{m \pi c t}{l} .
\end{aligned}
$$

This satisfies the differential equation, and makes $y=0$ for $t=0$. It only remains so to determine the constants that the series

$$
B_{1} \sin \frac{\pi x}{l}+B_{2} \sin \frac{2 \pi x}{l}+\ldots+B_{m} \sin \frac{m \pi x}{l},
$$

where

$$
B_{s}=\frac{s \pi c}{l} A_{s}
$$

may represent the initial distribution (1) of velocity, as nearly as possible. The determination of $B_{s}$ has virtually been made in $§ 32$ (15). With the necessary modifications of notation we find

$$
A_{8}=\frac{8 \beta_{0}}{\pi^{2}} \cdot \frac{1}{s^{2}}
$$

as stated in $§ 27$. The graph of the initial velocity, and the approximation attained by taking the first eight terms of the series (3), are shewn in Fig. 36.

It will be noticed that our approximation has even an advantage over the result obtained by carrying the series to infinity. In the latter case, the initial velocity, as represented by (1), is discontinuous when $x=0$, being zero for $x=0$, but equal to $4 \beta_{0} c / l$ when $x$ differs ever so little from 0 . The idealized representation of the motion in $\$ 27$ is in this respect imperfect; the parabola in Fig. 32 should be slightly modified so as to touch the line $A B$ at its extremities.

\section{String Excited by Impact.}

As a final example we take the case of a string started by an impact, as in $\S 26$. We begin with the case of a force 
distributed continuously in space and in time, the differential equation being

$$
\frac{\partial^{2} y}{\partial t^{2}}=c^{2} \frac{\partial^{2} y}{\partial x^{2}}+\frac{Y}{\rho}
$$

as in $§ 28$. Suppose, in the first place, that

$$
\frac{Y}{\rho}=f_{1}(t) \sin \frac{\pi x}{l}+f_{2}(t) \sin \frac{2 \pi x}{l}+\ldots+f_{m}(t) \sin \frac{m \pi x}{l},
$$

the coefficients being known functions of $t$. The equation (1) is then satisfied by

$$
y=\eta_{1} \sin \frac{\pi x}{l}+\eta_{2} \sin \frac{2 \pi x}{l}+\ldots+\eta_{m} \sin \frac{m \pi x}{l},
$$

provided

$$
\frac{\partial^{2} \eta_{8}}{\partial t^{2}}+\frac{s^{2} \pi^{2} c^{2}}{l^{2}} \eta_{s}=f_{s}(t)
$$

The solution of this equation has been given in $\S 8$. If we assume that $\eta_{s}=0, \dot{\eta}_{s}=0$ for $\dot{t}=-\infty$, and that $f_{s}(t)$ is sensible only for a finite range of $t$, the resulting value of $\eta_{s}$ is

$$
\begin{aligned}
\eta_{s}=\frac{l}{s \pi c} \sin \frac{s \pi c t}{l} \int_{-\infty}^{\infty} f_{s}(t) \cos \frac{s \pi c t}{l} d t \\
\quad-\frac{l}{s \pi c} \cos \frac{s \pi c t}{l} \int_{-\infty}^{\infty} f_{s}(t) \sin \frac{s \pi c t}{l} d t .
\end{aligned}
$$

If as a particular case we put

$$
f_{8}(t)=\frac{C_{8}}{\pi} \cdot \frac{\tau}{t^{2}+\tau^{2}},
$$

we have

$$
\eta_{s}=\frac{C_{s} l}{s \pi c} e^{-s \pi c \tau l l} \sin \frac{s \pi c t}{l},
$$

by $\S 8$ (18). As a function of $t, Y$ now follows the special law indicated by the last factor in (6), at every point of the string, but we have not yet made any special assumption as to the distribution of the force over the length. Its time-integral is given by

$$
\frac{1}{\rho} \int_{-\infty}^{\infty} Y d t=C_{1} \sin \frac{\pi x}{l}+C_{2} \sin \frac{2 \pi x}{l}+\ldots+C_{m} \sin \frac{m \pi x}{l} .
$$

We may now seek to determine the values of the coefficients so that this expression may be sensible only in the neighbourhood of the point $x=a$. We assume, then, that

$$
\frac{1}{\rho} \int_{-\infty}^{\infty} Y d t=\phi(x),
$$


where $\phi(x)$ vanishes except between the limits $a-\epsilon$ and $a+\epsilon$, say. The formula (7) of $\$ 36$ then gives

$$
C_{8}=\frac{2}{l} \int_{0}^{l} \phi(x) \sin \frac{s \pi x}{l} d x=\frac{2}{l} \int_{a-e}^{a+e} \phi(x) \sin \frac{s \pi x}{l} d x .
$$

If $\epsilon$ be small, the series thus obtained converges at first very slowly, and a great many terms might have to be taken to secure a reasonable approximation. In the terms of lower order we have

$$
C_{s}=\frac{2}{l} \sin \frac{s \pi a}{l} \int_{a-\mathrm{e}}^{a+e} \phi(x) d x=\frac{2 \mu}{\rho l} \sin \frac{s \pi a}{l},
$$

nearly, where

$$
\mu=\rho \int_{a-e}^{a+e} \phi(x) d x,
$$

i.e. $\mu$ represents the total impulse. The corresponding term in the value (3) of $y$ is

$$
\frac{2 \mu}{\rho \pi c} \cdot \frac{1}{s} e^{-8 \pi c \tau / l} \cdot \sin \frac{s \pi a}{l} \cdot \sin \frac{s \pi x}{l} \cdot \sin \frac{s \pi c t}{l} .
$$

But however small $\epsilon$ may be, so long as it is not evanescent, the value of $C_{8}$ given by (10) will ultimately tend to zero with increasing $s$, owing to the more and more complete mutual destruction of positive and negative elements under the integral sign. This shews the effect of diffusing the impulse over a small but finite portion of the string.

The case of an instantaneous local impulse is obtained by putting $\tau=0$ (cf. $§ 26$ ).

\section{General Theory of Normal Functions. Har-} monic Analysis.

The space which has been devoted to Fourier's theorem is no more than is warranted by its importance, especially in relation to the theory of strings, but it is well to remember that from the standpoint of the theory of vibrations the theorem is only one out of an infinite number which can be based on the same kind of physical considerations. Every vibratory system has its own series of "normal functions," as they are called, which express the configuration of the system in the various normal modes. In the case of a uniform string, or of the doubly-open organ pipe, these functions happen to have he simple form 
$\sin (s \pi x / l)$, or $\cos (s \pi x / l)$, respectively. More complicated forms will be met with when we come to the theory of transverse vibrations of bars, and to that of membranes; and even in the cases just mentioned the simplicity of type would at once disappear if the uniformity of line-density, or of cross-section, respectively, were departed from. In some problems, indeed, of considerable interest, e.g. that of the vibrations of a rectangular plate, the precise form of the functions has still to be discovered. But in any case the functions theoretically exist; and on the principle that any free motion whatever of the system consists of some combination or other of the various normal modes, it must be possible to express any arbitrary initial state, and therefore any arbitrary function of position in the system, by a series of normal functions. Such preeminence as attaches to Fourier's theorem is, from the present point of view, due merely to the fact that in it we have the simplest exemplification of this principle in the case of a continuous system, and the one where the physical induction has been most fully corroborated by independent mathematical proof. It may also be added that it is only in the case of strings that the calculation of the effect of particular initial conditions has any great interest.

There is however another point of view from which the resolution of a function into a series of sines or cosines of the variable is of peculiar importance, viz. when we are dealing with functions of the time. The dynamical reason for this has already been dwelt upon $(\$ 19)$.

When a function $f(t)$ is known to be periodic, of period $\tau$, its resolution by Fourier's theorem is

$$
\begin{aligned}
f(t)=A_{0} & +A_{1} \cos \frac{2 \pi t}{\tau}+A_{2} \cos \frac{2 \pi t}{\tau}+A_{3} \cos \frac{3 \pi t}{\tau}+\ldots \\
& +B_{1} \sin \frac{2 \pi t}{\tau}+B_{2} \sin \frac{2 \pi t}{\tau}+B_{3} \sin \frac{3 \pi t}{\tau}+\ldots
\end{aligned}
$$

where

$$
A_{0}=\frac{1}{\tau} \int_{0}^{\tau} f(t) d t
$$

whilst for $s>0$,

$$
A_{8}=\frac{2}{\tau} \int_{0}^{\tau} f(t) \cos \frac{2 s \pi t}{\tau} d t, \quad B_{8}=\frac{2}{\tau} \int_{0}^{\tau} f(t) \sin \frac{2 s \pi t}{\tau} .
$$


This is of course merely a restatement of the theorem of $\S 34$, with the necessary changes of notation. It will be noticed that $A_{0}$ represents the mean value of the function.

We have already been led to formulae of the type (1) as expressing the motion at any assigned point of a freely vibrating string, the period $\tau$ being equal to $2 l / c$. Another important acoustical application is to the analysis of a periodic current of air, as in the siren, or the reed-stops of an organ ( $(90)$. Again, in the case of electromagnetically driven tuning forks, a periodic current can powerfully excite, not only a fork in unison with itself, but also others whose natural frequencies are respectively, twice, three times, ... as great. This is due to the fact that the disturbing force is of the type (1), the selective resonance taking place according to the principles of $\S 9$.

Various mechanical contrivances for resolving a given periodic curve into its simple-harmonic constituents, and conversely for compounding a number of independent sineand cosine-curves whose periods are as $1, \frac{1}{2}, \frac{1}{3}, \ldots$, have been devised by Lord Kelvin and others. From the standpoint of the present subject the most remarkable of these is perhaps the machine constructed by Prof. A. A. Michelson, in which provision is made for as many as 80 constituents*.

It is hardly necessary to say explicitly that the resolution of a periodic function of $t$ in the form (1) can only be effected in one way, the values of the coefficients as given by (2) and (3) being determinate. In particular, a series of the above type cannot vanish for all values of $t$ unless its coefficients severally vanish. Thus in a freely vibrating string, if the motion at any given point $x$ be prevented, as by touching with a camel-hair pencil, the coefficients of $\cos (s \pi c t / l)$ and $\sin (s \pi c t / l)$ in the general formula (7) of $\$ 25$ must be zero, i.e. we must have

$$
A_{8} \sin \frac{s \pi x}{l}=0, \quad B_{8} \sin \frac{s \pi x}{l}=0
$$

for all values of $s$. Unless $x$ be commensurable with $l$ this requires that $A_{s}=0, B_{s}=0$, and the whole string will be

* Phil. Mag. (5), vol. xuv. (1898); this paper contains a number of most interesting examples of results obtained. The construction is also explained in his book On Light Waves and their Uses, Chicago, 1903. 
reduced to rest. In the excepted case the conditions (4) are satisfied independently of the values of $A_{s}$ and $B_{8}$ whenever $\sin (s \pi x / l)=0$, i.e. those normal modes remain unaffected which have a node at the point touched.

A question arises as to the effect of non-periodic forces on a dynamical system. For the reason already so often insisted upon, it is convenient, whenever possible, to resolve the force into a series of terms of the type

$$
A \cos p t+B \sin p t \text {. }
$$

Each element $A \cos p t$ or $B \sin p t$ then produces throughout the system its own effect, viz. an oscillation of the same type and period, the configuration and its amplitude depending on the speed $p$. In some cases the resolution presents itself quite naturally, as for example in the theory of the tides. The disturbing effect of the sun and moon, when account is taken of their varying declinations, and of the inequalities in their orbital motions, can be sufficiently represented by a series of terms of the type (5). It follows that the tide-height at any particular place must be expressed by a series of like character, in which the values of $p$ are known. The theoretical determination of the coefficients is out of the question for the actual ocean, with its variable depth and irregular boundaries, but their values can be inferred a posteriori with more or less accuracy from a comparison of the formula with observation, and when once ascertained can be used for prediction*.

When the disturbing force is perfectly arbitrary in character, without any obvious periodic elements, the question is more complicated. There is a form of Fourier's theorem specially appropriate to this case, but its application is usually difficult, and it is simpler to have recourse, as in $\$ 38$, to the formula (12) of $\S 8$. The objection that this implies a knowledge of the whole previous history of the system is met if we introduce the consideration of damping, which is in reality always present. The equation

$$
\frac{d^{2} x}{d t^{2}}+k \frac{d x}{d t}+n^{2} x=f(t)
$$

* For an elementary account of the matter see Sir G. H. Darwin, The Tides, London, 1898. 
may be written

$$
\left(\frac{d^{2}}{d t^{2}}+n^{\prime 2}\right) e^{\frac{1}{2} k t} x=e^{\frac{1}{2} k t} f(t)
$$

provided

$$
n^{\prime 2}=n^{2}-\frac{1}{4} k^{2}
$$

Hence, by the formula referred to,

$$
\begin{aligned}
x=\frac{1}{n^{\prime}} e^{-\frac{1}{2} k t} \sin n^{\prime} t \int e^{\frac{1}{2} k t} f(t) \cos n^{\prime} t d t & \\
& -\frac{1}{n^{\prime}} e^{-\frac{1}{2} k t} \cos n^{\prime} t \int e^{\frac{1}{2} k t} f(t) \sin n^{\prime} t d t .
\end{aligned}
$$

If we have $x=0, \dot{x}=0$ for $t=-\infty$, the limits of integration are $-\infty$ and $t$. For instance, the value of $x$ when $t=0$ becomes

$$
x=-\frac{1}{n^{\prime}} \int_{-\infty}^{0} e^{\frac{1}{2} k t} f(t) \sin n^{\prime} t d t .
$$

Owing to the presence of the exponential factor it is only for a certain range of negative values of $t$ that the function under the integral sign has as a rule an appreciable value. In other words, the effects of the action of the force prior to a certain antecedent epoch have practically disappeared. 


\section{CHAPTER IV}

BARS

\section{Elementary Theory of Elasticity. Strains.}

We require a few elementary notions from the theory of elasticity. As regards the purely geometrical study of deformations, or "strains," it is usual to begin with the "consideration of a body in a state of uniform or "homogeneous" strain. This is sufficiently defined by the property that any two lines in the substance which were originally straight and parallel remain straight and parallel, although their direction relative to other lines in the substance is usually altered. A parallelogram therefore remains a parallelogram, and it easily follows that the lengths of all finite parallel straight lines are altered in the same ratio; this ratio will however usually be different for different directions in the substance.

It can be shewn that there are three mutually perpendicular directions in the substance which remain mutually perpendicular after the deformation; these are called the "principal axes" of the strain. It is unnecessary, for our purposes, to give the formal proof, as the existence of such axes will be in evidence in such simple cases as we shall meet with. It follows from this theorem that any originally spherical portion of the substance is deformed into an ellipsoid whose axes are in the directions of the principal strain-axes.

If $P Q, P^{\prime} Q^{\prime}$ denote any straight line in the substance, before and after the strain, the ratio of the increase of length to the original length, viz.

$$
\epsilon=\frac{P^{\prime} Q^{\prime}-P Q}{P Q},
$$


is called the "extension"; it will in general be different for different directions of $P Q$. In the theory of elastic solids, $\epsilon$ is always a very minute fraction. We denote by $\epsilon_{1}, \epsilon_{2}, \epsilon_{3}$, the extensions in the directions of the principal axes.

The ratio of the increase of volume to the original volume is called the "dilatation." Denoting it by $\Delta$, and considering the change of volume of a cubical block whose edges are along the principal axes, we have

$$
1+\Delta=\left(1+\epsilon_{1}\right)\left(1+\epsilon_{2}\right)\left(1+\epsilon_{3}\right)
$$

or

$$
\Delta=\epsilon_{1}+\epsilon_{2}+\epsilon_{3},
$$

the products of small quantities being neglected.

There are two special types of homogeneous strain which require notice. First, suppose $\epsilon_{1}=\epsilon_{2}=\epsilon_{3},=\epsilon$, say. Any originally-spherical portion of the substance then remains spherical, and the extension is therefore the same in all directions. The strain may accordingly be described as one of uniform extension; and we note that $\epsilon=\frac{1}{3} \Delta$.

Again, take the case of $\epsilon_{1}=-\epsilon_{2}$, $=\epsilon$, say, whilst $\epsilon_{3}=0$, and therefore $\Delta=0$. A square whose diagonals $A O C, B O D$ are parallel to the axes

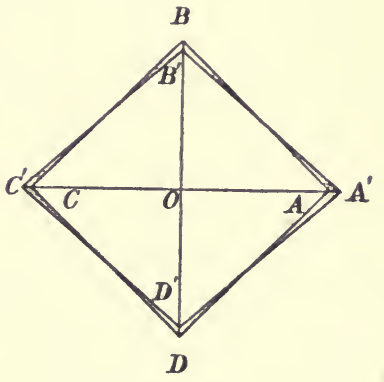

Fig. 38. 1,2 is converted into a rhombus $A^{\prime} B^{\prime} C^{\prime} D^{\prime}$, and since

$$
(1+\epsilon)^{2}+(1-\epsilon)^{2}=2,
$$

to the first order, the lengths of the sides are unaltered. Also

$$
\tan A^{\prime} O B^{\prime}=\frac{1+\epsilon}{1-\epsilon}=\tan \left(\frac{1}{4} \pi+\epsilon\right),
$$

so that the angles of the rhombus are $\frac{1}{2} \pi \pm 2 \epsilon$. Another view of this state of strain is obtained if we imagine the rhombus $A^{\prime} B^{\prime} C^{\prime} D^{\prime}$ to be moved in its own plane so that $A^{\prime} B^{\prime}$ coincides with $A B$. This is legitimate, since no displacement of the body as a whole affects the question. We then see that any two planes of the substance parallel to $A B$ and the axis 3 are displaced relatively to one another, without change of mutual 
distance, by an amount proportional to this distance. This kind of strain is called a "shear," from the fact that it is of the type which tends to be set up by the action of the two edges of a pair of shears. The "amount" $(\eta)$ of the shear is specified by the relative displacement per unit of mutual distance, i.e. by the ratio $D D^{\prime} / A D$, or $2 \epsilon$, in the first part of Fig. 39. Again, by moving $B^{\prime} C^{\prime}$ into coincidence with $B C$, we might prove that the strain is also equivalent to a shearing of planes parallel to $B C$ and the axis 3 , in the direction of $B C$. This is shewn in the second half of the figure.
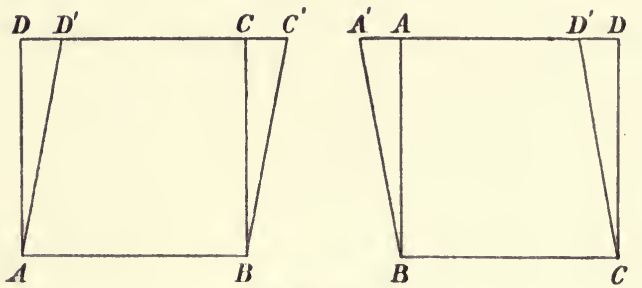

Fig. 39 .

\section{Stresses.}

The name "stress" is applied to the mutual action which is exerted across any ideal surface $S$ drawn in a body, between the portions of matter immediately adjacent to $S$ on either side. We are here concerned with molecular actions sensible only over an exceedingly short range, so that the portions of matter in question are confined to two exceedingly thin strata, whose common boundary is $S$. The resultant force on a small portion of either stratum may then be taken to be ultimately proportional to its area, and the intensity of the stress is accordingly specified by the force per unit area. This force may be of the nature either of a push or a pull, and may be normal or oblique, or even tangential to the area.

For simplicity, it is usual to begin with the notion of a state of uniform or "homogeneous" stress, i.e. the stress over any plane is assumed to be uniform, and the same in direction and intensity for any two parallel planes. It will of course in general be different for planes drawn in different directions. It may be shewn that there are then three mutually perpendic- 
ular sets of planes such that across each of these the stress is in the direction of the normal ; but for a reason already indicated we need not stop to prove this theorem. The planes in question are called the "principal planes" of the stress, and the corresponding stress-intensities are called the "principal stresses." They are usually reckoned as positive when of the nature of tensions; we denote them by $p_{1}, p_{2}, p_{3}$.

There are certain special types of stress to be noticed. First let $p_{1}=p_{2}=p_{3}$. The stress across every plane is then in the direction of the normal, and of uniform intensity, as in hydrostatics.

Next, let $p_{1}=-p_{2},=p$, say, whilst $p_{3}=0$. Consider a unit cube whose faces are parallel to the principal planes. The portion included between the faces represented by $A B, D A$, in the figure, and the diagonal plane represented by $B D$, is in equilibrium under three forces. Two of these forces are parallel and proportional to $D A$ and $A B$, viz. the forces on $A B$ and $D A$, respectively. The third force is therefore along and

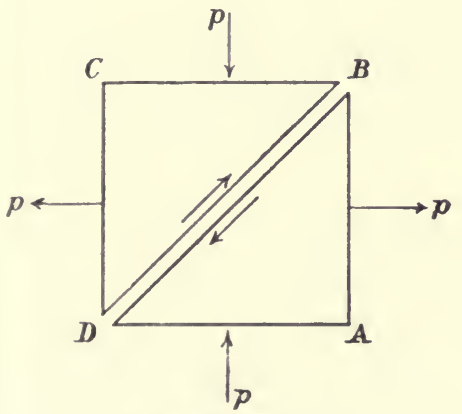

Fig. 40.

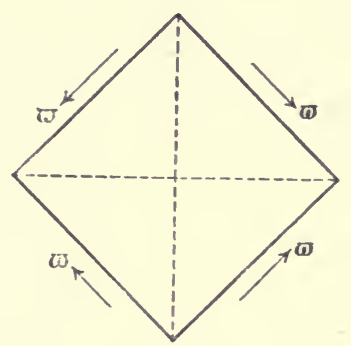

Fig. 41.

proportional to $B D$; and its amount $(\varpi)$ per unit area is $p$. A similar result holds with respect to the diagonal plane $A C$. A cube four of whose faces are parallel to these diagonal planes is in equilibrium under tangential stresses, in the manner shewn. This type is accordingly called a "shearing stress." Its amount $(\varpi)$ is specified by the tangential force per unit area on the planes in question. 
In general the states of strain and stress in a body are not uniform, or "homogeneous," but vary continuously from point to point; but the above notions are still applicable to the infinitely small elements into which the body may be conceived to be divided.

\section{Elastic Constants. Potential Energy of Defor- mation.}

The theory of strains is a matter of pure geometry; that of stresses one of pure statics. When we come to connect the two we require some physical assumption. The usual hypothesis, known as "Hooke's law,"* is that the stresses are linear functions of the strains. This law ceases to hold, even approximately, when the strains exceed certain values called the "elastic limits"; but for the purposes of acoustics it may be adopted without hesitation, on account of the excessive minuteness of the varying strains with which we are concerned $\dagger$.

In an "isotropic" substance, i.e. one in which there is no distinction of properties between one direction and another, the principal axes of strain must evidently coincide with those of stress. Moreover the principal stress $p_{1}$ must involve the principal strains $\epsilon_{2}, \epsilon_{3}$ symmetrically, and so on. The most general assumption consistent with this requirement, and with Hooke's law, is of the form

$$
\left.\begin{array}{l}
p_{1}=\lambda\left(\epsilon_{1}+\epsilon_{2}+\epsilon_{3}\right)+2 \mu \epsilon_{1}, \\
p_{2}=\lambda\left(\epsilon_{1}+\epsilon_{2}+\epsilon_{3}\right)+2 \mu \epsilon_{2}, \\
p_{3}=\lambda\left(\epsilon_{1}+\epsilon_{2}+\epsilon_{3}\right)+2 \mu \epsilon_{3},
\end{array}\right\}
$$

where $\lambda, \mu$ are constants depending on the nature of the material ${ }_{+}^{+}$. It will be noticed that $\epsilon_{1}, \epsilon_{2}, \epsilon_{3}$ are pure ratios and that the dimensions of $\lambda, \mu$ are therefore those of stress, or force

* Robert Hooke (1635-1703), professor of geometry at Gresham College $1665-1703$.

+ If Hooke's law were sensibly departed from, the frequencies of the normal modes of a vibrating bar would no longer be independent of the amplitude. Since the ear is very sensitive to variation of pitch, this would easily be detected. This remark is due to Stokes.

\# There is a great diversity of notation as regards these constants. The above symbols are those introduced by G. Lamé (1795-1870), professor of physics at the École polytechnique 1832-44. 
divided by area, viz. $\left[M L^{-1} T^{-2}\right]$ if $[M],[L],[T]$ denote the units of mass, length, and time.

There are various combinations of the constants $\lambda, \mu$ which are important in physics, as well as in technical mechanics. In a uniform dilatation we have $p_{1}=p_{2}=p_{3}$ ( $=p$, say), $\epsilon_{1}=\epsilon_{2}=\epsilon_{3}$ $\left(=\frac{1}{3} \Delta\right)$, whence

Hence if we write

$$
\begin{gathered}
p=\left(\lambda+\frac{2}{3} \mu\right) \Delta . \\
\kappa=\lambda+\frac{2}{3} \mu,
\end{gathered}
$$

$\kappa$ will denote the "volume-elasticity" or "cubical elasticity" of the substance, i.e. the ratio of the uniform stress to the dilatation which it involves.

Next suppose that $\epsilon_{1}=-\epsilon_{2}=\epsilon, \epsilon_{3}=0$, and therefore $p_{1}=-p_{2}=p, p_{3}=0$, which is the case of a pure shear, involving a shearing stress. According to the investigations of $\$ 40,41$ the shearing stress is $\varpi=p$, and the shear is $\eta=2 \epsilon$. Hence, from (1),

$$
\varpi=\mu \eta,
$$

i.e. $\mu$ denotes the ratio of the stress to the strain (appropriately measured) in a pure shear. It is called the "rigidity" of the substance.

Again, suppose we have a bar stretched lengthways, but free from lateral stress. We put, then, in (1), $p_{2}=0, p_{3}=0$. This leads to

$$
p_{1}=E \epsilon_{1}
$$

where

$$
E=\frac{(3 \lambda+2 \mu) \mu}{\lambda+\mu}=\frac{3 \kappa \mu}{\lambda+\mu} .
$$

This ratio of the longitudinal stress to the corresponding extension is called "Young's modulus" of elasticity; its technical importance is obvious. We also find

$$
\epsilon_{2}=\epsilon_{3}=-\sigma \epsilon_{1},
$$

where

$$
\sigma=\frac{\lambda}{2(\lambda+\mu)} .
$$

This fraction accordingly measures the ratio of lateral contraction to longitudinal extension under the circumstances supposed; it is known as "Poisson's ratio."*

* S. D. Poisson (1781-1840). His chief contributions to acoustics relate to the vibrations of membranes and plates, and to the general theory of soundwaves in air. 
By solving the equations (1) we can express $\epsilon_{1}, \epsilon_{2}, \epsilon_{3}$ as linear functions of $p_{1}, p_{2}, p_{3}$. It is obvious, however, that the formula for $\epsilon_{1}$ must involve $p_{2}$ and $p_{3}$ symmetrically; and from this consideration, and from the physical meanings of the constants $E$ and $\sigma$, it follows immediately that the result must be equivalent to

$$
\left.\begin{array}{l}
E \epsilon_{1}=p_{1}-\sigma\left(p_{2}+p_{3}\right), \\
E \epsilon_{2}=p_{2}-\sigma\left(p_{3}+p_{1}\right), \\
E \epsilon_{3}=p_{3}-\sigma\left(p_{1}+p_{2}\right) .
\end{array}\right\}
$$

Of the various elastic constants and their combinations, one or other may appear specially important, according to the nature of the question in view, and this may account for the great diversity of notations which has arisen. In any case two independent quantities are necessary and sufficient to define the elastic behaviour of an isotropic substance. From a physical standpoint $\kappa$ and $\mu$ might appear to be the most fundamental; whilst as regards facility of direct measurement preference may be given to $E$ and $\mu$, whence $\kappa$ and $\sigma$ can be derived by the formulae

$$
\kappa=\frac{\mu E}{9 \mu-3 E}, \quad \sigma=\frac{E}{2 \mu}-1,
$$

which follow easily from (3), (6) and (7). On a particular hypothesis as to the ultimate structure of an elastic solid Poisson was led to the conclusion that the two elastic constants are not independent, but are connected by an invariable relation, which in our notation is expressed by $\lambda=\mu$. This makes

$$
\kappa=\frac{5}{3} \mu, \quad E=\frac{5}{2} \mu, \quad \sigma=\frac{1}{4} .
$$

On experimental grounds Wertheim (1848) proposed the relation $\lambda=2 \mu$, which makes

$$
\kappa=\frac{8}{3} \mu, \quad E=\kappa, \quad \sigma=\frac{1}{3} .
$$

More accurate methods of measurement, introduced by Kirchhoff* and others, support the view, which has been consistently held by English physicists $\uparrow$ that there is no necessary

* G. R. Kirchhoff (1824-87), professor of physics at Heidelberg 1854-75, at Berlin 1875-87; famous for his share in the discovery of spectrum analysis, but the author also of important memoirs on the theory of elasticity and its applications to the vibrations of bars and plates.

+ Notably by Green (1793-1841), Stokes, and Lord Kelvin. 
definite relation between $\lambda$ and $\mu$, and consequently no universal value of $\sigma$. We may note that in an absolutely incompressible medium we should have

$$
\kappa=\infty, E=3 \mu, \quad \sigma=\frac{1}{2} .
$$

The following table gives the results of a few determinations by Everett (1867). The second column gives the volumedensity in grammes per cubic centimetre. The next three columns give the respective elastic constants, in dynes per square centimetre. These are followed in the last column by the corresponding values of $\sigma$. The last two rows illustrate the fact that the elastic constants may vary appreciably in different specimens of nominally the same substance.

\begin{tabular}{|l|c|c|c|c|c|}
\hline & $\rho$ & $E$ & $\mu$ & $\kappa$ & $\sigma$ \\
\hline Steel & $7 \cdot 849$ & $2.139 \times 10^{12}$ & $8 \cdot 19 \times 10^{11}$ & $1 \cdot 841 \times 10^{12}$ & $\cdot 310$ \\
Iron (wrought) & $7 \cdot 677$ & $1.963 \times 10^{12}$ & $7 \cdot 69 \times 10^{11}$ & $1 \cdot 456 \times 10^{12}$ & $\cdot 275$ \\
Iron (cast) & $7 \cdot 235$ & $1 \cdot 349 \times 10^{12}$ & $5.32 \times 10^{11}$ & $\cdot 964 \times 10^{12}$ & $\cdot 267$ \\
Copper & $8 \cdot 843$ & $1 \cdot 234 \times 10^{12}$ & $4 \cdot 47 \times 10^{11}$ & $1 \cdot 684 \times 10^{12}$ & $\cdot 378$ \\
Glass (1) & $2 \cdot 942$ & $\cdot 603 \times 10^{12}$ & $2 \cdot 40 \times 10^{11}$ & $\cdot 415 \times 10^{12}$ & $\cdot 258$ \\
Glass (2) & $2 \cdot 935$ & $\cdot 574 \times 10^{12}$ & $2.35 \times 10^{11}$ & $\cdot 347 \times 10^{12}$ & $\cdot 229$ \\
\hline
\end{tabular}

For technical purposes the elastic constants $\boldsymbol{E}, \kappa, \mu$ are often expressed in gravitation measure, e.g. in grammes per square centimetre. The corresponding numbers in the above table are then divided by $g$. Another mode of specification, employed by Young, is in terms of the length of a bar of the particular substance, whose weight per unit area of cross-section would be equal to the modulus in question when expressed in gravitation measure; this is called the "length-modulus." Thus if $L$ be the length-modulus of extension of a bar free to contract laterally we have

$$
E=g \rho L
$$

Taking $g=981$, the above table gives, in the case of steel, $L=278 \times 10^{6}$ centimetres. 
The potential energy $(W)$ per unit volume of a strained isotropic substance may be found by calculating the work done by the stresses on the faces of a unit cube, on the hypothesis that the strains increase from zero to their final values keeping their mutual ratios unchanged. The average stresses are then one-half the final stresses.

Thus in the case of a uniform dilatation $\Delta$ we have

$$
W=\frac{1}{2} p \Delta=\frac{1}{2} \kappa \Delta^{2} .
$$

In the case of a pure shear $\eta$,

$$
W=\frac{1}{2} \varpi \eta=\frac{1}{2} \mu \eta^{2} .
$$

In the extension of a bar, with freedom of lateral contraction,

$$
W=\frac{1}{2} p_{1} \epsilon_{1}=\frac{1}{2} E \epsilon_{1}^{2} .
$$

In the general case we have

$$
\begin{aligned}
W & =\frac{1}{2}\left(p_{1} \epsilon_{1}+p_{2} \epsilon_{2}+p_{3} \epsilon_{3}\right) \\
& =\frac{1}{2} \lambda\left(\epsilon_{1}+\epsilon_{2}+\epsilon_{3}\right)^{2}+\mu\left(\epsilon_{1}{ }^{2}+\epsilon_{2}{ }^{2}+\epsilon_{3}{ }^{2}\right) \\
& =\frac{1}{2} \kappa \Delta^{2}+\frac{1}{3} \mu\left\{\left(\epsilon_{2}-\epsilon_{3}\right)^{2}+\left(\epsilon_{3}-\epsilon_{1}\right)+\left(\epsilon_{1}-\epsilon_{2}\right)^{2}\right\} .
\end{aligned}
$$

This shews that in order that the potential energy may be a minimum in the unstrained state $\kappa$ and $\mu$ must be positive. It is otherwise obvious from the meaning of the symbols that if either of these were negative the unstrained state would be unstable.

\section{Longitudinal Vibrations of Bars.}

We take the axis of $x$ along the bar, and denote by $x+\xi$ the position at time $t$ of that cross-section whose undisturbed position is $x$, so that $\xi$ denotes the displacement. An element of length is then altered from $\delta x$ to $\delta(x+\xi)$, or $\left(1+\xi^{\prime}\right) \delta x$, where the accent denotes differentiation with respect to $x$. Equating this to $(1+\epsilon) \delta x$, we have

$$
\epsilon=\frac{\partial \xi}{\partial x}
$$

The tension across the sectional area $(\omega)$ is therefore $E \epsilon \omega$. The acceleration of momentum of the mass included between the two cross-sections corresponding to $x$ and $x+\delta x$ is $\rho \omega \delta x . \ddot{\xi}$. 
Equating this to the difference of the forces on the two ends, we have

or

$$
\begin{gathered}
\rho \omega \delta x \cdot \frac{\partial^{2} \xi}{\partial t^{2}}=\delta\left(E \omega \frac{\partial \xi}{\partial x}\right), \\
\rho \omega \frac{\partial^{2} \xi}{\partial t^{2}}=E \frac{\partial}{\partial x}\left(\omega \frac{\partial \xi}{\partial x}\right) .
\end{gathered}
$$

If the section be uniform, this reduces to

$$
\frac{\partial^{2} \xi}{\partial t^{2}}=c^{2} \frac{\partial^{2} \xi}{\partial x^{2}},
$$

where

$$
c^{2}=E / \rho \text {. }
$$

It will be noticed that in this investigation it is not necessary to assume the substance of the bar to be isotropic, provided the proper value of the Young's modulus be taken*.

-The result is also unaffected if the bar, or wire, be subject to a permanent longitudinal tension, since by Hooke's law the stress due to the extension (1) may be superposed on the permanent tension, so long as the limits of perfect elasticity are not transgressed.

As in $§ 23$ the general solution of (3) is

$$
\xi=f(c t-x)+F(c t+x),
$$

representing two wave-systems travelling unchanged in opposite directions with the velocity $c$ given by (4). In terms of the length-modulus, we have by $§ 42$ (14)

$$
c=\sqrt{ }(g L)
$$

this is the velocity due to a fall from rest through a height $\frac{1}{2} L$. Some numerical values of $c$ are given in the last column of the table on p. 119.

The application to particular problems may be treated very briefly. The various cases that arise present themselves in a more interesting form when we come to the vibrations of - columns of air.

In the case of a rod or wire fixed at both ends, we have $\xi=0$ for $x=0$ and $x=l$ (say); and the mathematical theory

* In an "aelotropic" or crystalline solid the values of $E$ will be different for bars cut in different directions from the substance. 
is exactly the same as in the case of the transverse vibrations of a string. The frequencies of the various modes are given by

$$
N=s c / 2 l \text {, }
$$

where $s=1,2,3, \ldots$. The result is unaffected by permanent tension in the wire.

When the rod is free, the condition of zero stress at the ends gives $\xi^{\prime}=0$ for $x=0$ and $x=l$. Introducing this condition in (5) we find

$$
F^{\prime \prime}(c t)=f^{\prime}(c t), \quad F^{\prime}(c t+l)=f^{\prime}(c t-l),
$$

for all values of $t$. The former of these gives on integration

$$
F(c t)=f(c t) \text {, }
$$

no explicit additive constant being necessary since it may be supposed included in the value of $f(c t)$. The second relation then gives

$$
f(c t+l)=f(c t-l)+C .
$$

The constant $C$ is connected with the total momentum of the bar in the direction of its length. We have, from (9) and (10),

$$
\int_{0}^{l} \dot{\xi} d x=c \int_{0}^{l}\left\{f^{\prime}(c t-x)+f^{\prime}(c t+x)\right\} d x=c C .
$$

Since nothing essential is altered if we superpose any uniform velocity in the direction of the length, we may assume the mass-centre to be at rest, in which case $C=0$. The formula (10) then shews that the residual motion is periodic, since everything recurs when $t$ increases by $2 l / c$.

In the analytical process for ascertaining the normal modes we assume that $\xi$ varies as $\cos (n t+\epsilon)$, whence

$$
\frac{\partial^{2} \xi}{\partial x^{2}}+\frac{n^{2}}{c^{2}} \xi=0
$$

and $\quad \xi=\left(A \cos \frac{n x}{c}+B \sin \frac{n x}{c}\right) \cos (n t+\epsilon)$.

The conditions that $\partial \xi / \partial x=0$ for $x=0$ and $x=l$ require $B=0$, $\sin (n l / c)=0$, whence

$$
n l / c=s \pi,
$$

where $s=0,1,2,3, \ldots$, the scale of periods being harmonic. The nodes $(\xi=0)$ are given by $\cos (s \pi x / l)=0$, and the loops, 
or places of zero stress, by $\sin (s \pi x / l)=0$. In the gravest mode $(s=1)$ we have a node at the centre*.

On the principles explained in $\$ 16,32$ the most general free motion of the bar, under the present conditions, may be expressed by a series

$$
\xi=\Sigma\left(A_{s} \cos \frac{s \pi c t}{l}+B_{s} \sin \frac{s \pi c t}{l}\right) \cos \frac{s \pi x}{l},
$$

where $s=0,1,2,3, \ldots$ Thus if the bar be started from rest in the state of strain defined by

$$
\xi=f(x) \quad[t=0],
$$

we have $B_{s}=0$; and we infer that it must be possible to determine the coefficient $A_{8}$ so that

$$
f(x)=\Sigma A_{8} \cos \frac{s \pi x}{l}
$$

for values of $x$ ranging from 0 to $l$. This is the result referred to by anticipation in $\S 33$.

The longitudinal vibrations of bars or wires have hardly any practical application of importance, except in some primitive forms of telephone. As regards bars, the pitch is very high compared with that of the transverse vibrations, which it is difficult to avoid exciting simultaneously. Again, if we compare the frequencies of longitudinal vibration of a tense wire with those of the corresponding transverse modes, the ratio will be that of the wave-velocities, i.e. of $\sqrt{ }(E / \rho)$ to $\sqrt{ }(P / \rho \omega)$, where $P$ denotes the permanent tension. If $\epsilon_{0}$ be the extension due to $P$, we have $P=E \epsilon_{0} . \omega$, and the ratio is $1 / \sqrt{ } \epsilon_{0}$, which is usually very great $\dagger$. Longitudinal vibrations may be elicited on the monochord by rubbing the wire lengthwise with a piece of leather sprinkled with resin; the resulting note is very shrill.

It is assumed in the preceding theory that the extension and the accompanying stress are at any instant uniform over the cross-section; in other words, we have assumed that the

* The case $s=0$ needs, in strictness, separate examination. It leads to $\xi=A_{0}(1+a t)$, which may be interpreted as an oscillation of infinitely long period. If the mass-centre be at rest we have $A_{0}=0$.

+ This comparison is due to Poisson (1828). 
lateral contraction adjusts itself instantaneously through the thickness. This is not quite exact, as there is a certain degree of lateral inertia, but the error is insignificant so long as the wave-length is large compared with the diameter. In the modes of very high order it might become sensible, but these are in any case of no importance from the point of view of acoustics. - A correction has been investigated by Lord Rayleigh.

\section{Plane Waves in an Elastic Medium.}

The theory of plane waves in an unlimited isotropic elastic medium is so closely analogous to that of longitudinal waves in a rod that it may be briefly noticed here. It is assumed that the state of things is at any instant uniform over any plane perpendicular to the direction of propagation $(x)$.

Such waves may be of two types, which are distinguished as "dilatational" or "longitudinal," and "distortional" or "transversal," respectively. In the former class the displacement is wholly in the direction of propagation. Denoting it by $\xi$, we have, in the notation of $\S 42$,

$$
\epsilon_{1}=\partial \xi / \partial x, \quad \epsilon_{2}=0, \quad \epsilon_{3}=0,
$$

and therefore $p_{1}=(\lambda+2 \mu) \epsilon_{1}=\left(\kappa+\frac{4}{3} \mu\right) \partial \xi / \partial x$.

Considering the portion of matter corresponding to unit area of a stratum of thickness $\delta x$, we have

whence

$$
\rho \delta x . \ddot{\xi}=\delta p_{1},
$$

if

$$
\frac{\partial^{2} \xi}{\partial t^{2}}=a^{2} \frac{\partial^{2} \xi}{\partial x^{2}}
$$

Some numerical values of the wave-velocity $a$ are given on the next page, and it will be observed that they are in all cases greater than the corresponding values of $c$, as was to be expected, since the potential energy due to a given extension $\partial \xi / \partial x$ is now greater owing to the absence of lateral yielding.

In the second type of plane waves the displacement is everywhere at right angles to the direction of propagation. It may be resolved into two components parallel to $y$ and $z$, respectively, which may be treated separately. Considering 
the former component $(\eta)$ alone, we see that the strain at any point consists in a shear of amount $\partial \eta / \partial x$. The consequent stress across any plane perpendicular to $O x$ is parallel to $O y$, and its intensity is $\mu \partial \eta / \partial x$. Hence forming the equation of motion of a portion of matter defined as before we have

$$
\begin{array}{rlrl} 
& & \rho \delta x . \ddot{\eta}=\delta\left(\mu \eta^{\prime}\right), \\
\text { or } & \frac{\partial^{2} \eta}{\partial t^{2}}=b^{2} \frac{\partial^{2} \eta}{\partial x^{2}}, \\
\text { if } & b^{2}=\mu / \rho .
\end{array}
$$

Some values of the wave-velocity $b$ are tabulated below.

Wave-velocities (metres per second).

\begin{tabular}{|l|c|c|c|}
\hline & $a$ & $b$ & $c$ \\
\hline Steel & $6.11 \times 10^{3}$ & $3.23 \times 10^{3}$ & $5 \cdot 22 \times 10^{3}$ \\
Iron (wrought) & $5.68 \times 10^{3}$ & $3.16 \times 10^{3}$ & $5.06 \times 10^{3}$ \\
Iron (cast) & $4.81 \times 10^{3}$ & $2.71 \times 10^{3}$ & $4.32 \times 10^{3}$ \\
Copper & $5.08 \times 10^{3}$ & $2.25 \times 10^{3}$ & $3.74 \times 10^{3}$ \\
Glass (1) & $5.00 \times 10^{3}$ & $2.86 \times 10^{3}$ & $4.53 \times 10^{3}$ \\
Glass (2) & $4.74 \times 10^{3}$ & $2.83 \times 10^{3}$ & $4.42 \times 10^{3}$ \\
\hline
\end{tabular}

It may be shewn that any local disturbance in an unlimited elastic medium breaks up into two waves, diverging with the velocities $a$ and $b$, which tend ultimately to assume the "longitudinal" and "transverse" characters, respectively. The theory is historically important in relation to Optics, but in our present subject great caution is necessary in drawing inferences as to the propagation of waves in limited solids. We have already seen that in a cylindrical or prismatic rod the velocity of longitudinal waves is quite distinct from $a$, and the theory becomes altogether different in the case of flexural vibrations, to be referred to presently. In these cases a modification was of course to be expected, since the wavelength is understood to be large compared with the dimensions 
of the cross-section. But even in the other extreme, when all the dimensions of the body are large compared with the wavelength, the circumstances may be profoundly modified by the existence of a free boundary. A new type of waves, called after the discoverer the "Rayleigh waves" (1885), make their appearance, and under some conditions may become, from the observational point of view, predominant. These are surface waves in which the agitation penetrates only to a relatively small depth. Their velocity is somewhat less than that of the distortional waves; thus for an incompressible solid it is $9554 b$, whilst on Poisson's hypothesis $\left(\sigma=\frac{1}{4}\right)$ it is $9194 b$. In modern observations of the tremors due to distant earthquakes three phases of the disturbance are often recognized. The first is interpreted as due to the arrival of the dilatational waves, propagated directly through the substance of the earth, the second as due to that of the distortional waves, also propagated directly, and the third to that of the Rayleigh waves, which have travelled over the surface and are therefore delayed more than in proportion to the difference of wavevelocity*. The latter waves as they spread over the surface are less attenuated than the former, which diverge in three dimensions. It has even been attempted to deduce estimates of the volume-elasticity and rigidity of the materials of the earth from the various wave-velocities, as inferred from the seismic recordst.

45. Flexural Vibrations of a Bar.

We proceed to the transverse vibrations of a bar naturally straight. To avoid unnecessary complications we will suppose that the bar has a longitudinal plane of symmetry, and that the flexure takes place paralle! to this plane. We will also assume for the present that the total longitudinal stress on any section is zero. The resultant stress at a section there-

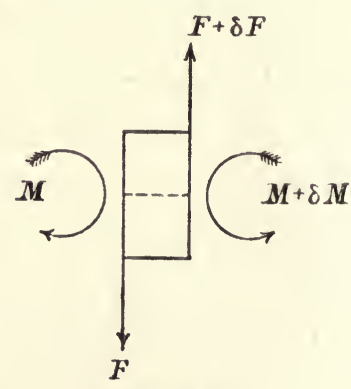

Fig. 42.

* R. D. Oldham, Phil. Trans. A, 1900.

+ Prof. A. E. H. Love, Phil. Trans. A, vol. ccrir., p. 215 (1908). 
fore reduces to a transverse "shearing force" $F$, and a couple or "bending moment" $M$. These will be functions of $x$, the longitudinal coordinate. If $\eta$ denote the lateral displacement, parallel to the plane of symmetry, then, resolving transversally the forces acting on an element $\delta x$ of the length, we have

$$
\begin{gathered}
\rho \omega \delta x . \ddot{\eta}=\delta F, \\
\rho \omega \frac{\partial^{2} \eta}{\partial t^{2}}=\frac{\partial F}{\partial x} .
\end{gathered}
$$

or

Again, if $\kappa$ denote the radius of gyration of the area of the cross-section $\omega$ about an axis through its centre of gravity, normal to the plane of flexure, the element of mass is ultimately a disk of area $\omega$, thickness $\delta x$, and moment of inertia $\rho \omega \delta x . \kappa^{2 *}$. Since the axis of this disk has been turned through a small angle $\partial \eta / \partial x$ from the position of equilibrium, the equation of angular motion is

$$
\begin{array}{r}
\rho \omega \delta x . \kappa^{2} \frac{\partial^{2}}{\partial t^{2}}\left(\frac{\partial \eta}{\partial x}\right)=\delta M+F \delta x, \\
\rho \omega \kappa^{2} \frac{\partial^{3} \eta}{\partial x \partial t^{2}}=\frac{\partial M}{\partial x}+F . \quad \ldots
\end{array}
$$

If we eliminate $F$ between (1) and (2) we have

$$
\rho \omega\left(\frac{\partial^{2} \eta}{\partial t^{2}}-\kappa^{2} \frac{\partial^{4} \eta}{\partial x^{2} \partial t^{2}}\right)=-\frac{\partial^{2} M}{\partial x^{2}}
$$

provided the sectional area $\omega$ be uniform.

We have next to express $M$ in terms of the deformation of the bar. Consider in the first instance the case of a bar uniformly bent, so that its axis becomes an arc of a circle. It is evident from symmetry that the shearing force $F$ now vanishes, and it hardly needs calculation to shew that the strain in any part of the cross-section will be proportional to the curvature. Hence by Hooke's law the resultant couple $M$ will also vary as the curvature, or

$$
M=\mathfrak{i b} / R,
$$

where $R$ is the radius of curvature, and $\mathcal{B}$ is a constant depending on the shape and size of the cross-section, and on the elastic properties of the material.

* The symbol $\kappa$ is not required at present in its former sense as an elastic constant. 
The value of $\mathfrak{Z B}$ is found as follows. We take rectangular axes $G y, G z$ in the plane of a section, the origin being at the centre (i.e. the centre of gravity of the area), and the axis of $z$ normal to the plane of flexure. Assuming the axis of the bar, i.e. the line through the centres of the sections, to be unextended, we see that if $R$ denote the radius of the circle into which it is bent, the length of a longitudinal linear element whose distance from the plane $x z$ is $y$ is altered in the ratio of $R+y$ to $R$, and that the extension is accordingly $y / R$. The corresponding stress per unit area of the section is $E y / R$,

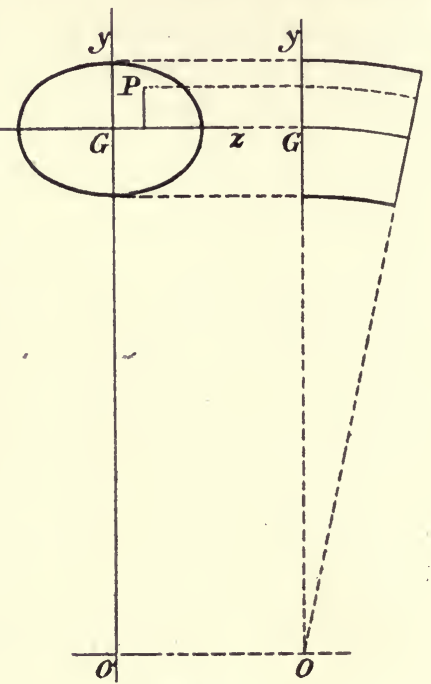

Fig. 43. where $E$ is the appropriate Young's modulus. The total longitudinal tension is therefore

$$
=\frac{E}{R} \iint y d y d z=0 \text {. }
$$

This justifies the provisional assumption that the axis (as above defined) is on the present hypothesis unextended. For the bending moment we have, taking moments about $G z$,

$$
M=\frac{E}{R} \iint y^{2} d y d z=\frac{E \omega \kappa^{2}}{R} .
$$

Except in the special case just considered, viz. that of a bar bent statically into an arc of uniform curvature, there will be a shearing of cross-sections relative to one another, and also a warping of the sections so that these do not remain accurately plane. An exact investigation is out of the question, but enough is understood of the matter to warrant the statement that the additional strains thus introduced are as a rule small compared with those taken account of in the preceding calculation. We therefore adopt the formula (5) as sufficiently 
accurate in all cases, provided $R$ denote the radius of curvature at the point considered.

In the present application $\partial \eta / \partial x$ is a small quantity, so that we may put $R^{-1}=\partial^{2} \eta / \partial x^{2}$, and therefore

$$
M=E \omega \kappa^{2} \frac{\partial^{2} \eta}{\partial x^{2}}
$$

Substituting in (3) we obtain

$$
\frac{\partial^{2} \eta}{\partial t^{2}}-\kappa^{2} \frac{\partial^{4} \eta}{\partial x^{2} \partial t^{2}}+\frac{E \kappa^{2}}{\rho} \frac{\partial^{4} \eta}{\partial x^{4}}=0 .
$$

For most purposes this equation may be simplified by the omission of the second term, as we shall see immediately.

The kinetic energy of the bar is

$$
T=\frac{1}{2} \rho \omega \int\left(\frac{\partial \eta}{\partial t}\right)^{2} d x+\frac{1}{2} \rho \omega \kappa^{2} \int\left(\frac{\partial^{2} \eta}{\partial x \partial t}\right)^{2} d x
$$

The second term, which represents the energy of rotation of the elements, is usually negligible.

The potential energy is found, in accordance with $§ 42(16)$, by integrating the expression $\frac{1}{2} E \epsilon^{2},=\frac{1}{2} E y^{2} / R^{2}$, first over the area of the cross-section, and then over the length; thus

$$
V=\frac{1}{2} E \omega \kappa^{2} \int\left(\frac{\partial^{2} \eta}{\partial x^{2}}\right)^{2} d x
$$

Consider for a moment the propagation of a system of waves of simple-harmonic profile along an unlimited rod, assuming

$$
\eta=C \cos k(c t-x) \text {. }
$$

Since everything here recurs whenever $x$ is increased by $2 \pi / k$, the constant $k$ is connected with the wave-length $\lambda$ by the relation

$$
k=2 \pi / \lambda \text {. }
$$

On substitution the equation (7) is found to be satisfied provided

$$
c^{2}=\frac{k^{2} \kappa^{2}}{1+k^{2} \kappa^{2}} \cdot \frac{E}{\rho} .
$$

This gives the wave-velocity $c$, which is seen not to be a definite quantity fixed by the constitution of the rod, but to depend also on the wave-length. To trace the progress of a wave of any type other than (10), it would be necessary to resolve the wave- 
form into simple-harmonic functions of $x$. Each of these would travel with its own velocity, so that the resultant wave-profile would continually alter. For this reason it would be hopeless to look for a general solution of (7), or even of the modified form (13) below, of the same simple character that we met with in the theory of strings ( $\$ 23)$, and again in that of the longitudinal vibrations of rods.

A further remark is that when we substitute from (10) in (7), the second term is of the order $k^{2} \kappa^{2}$ as compared with the first. When the wave-length is large compared with the dimensions of the cross-section this is a very small quantity, and the term in question, which arose through taking account of the rotatory inertia of the elements of the bar, viz. in equation (2), may be neglected. It is easy to see, and it may be verified $a$ posteriori, that the same simplification is legitimate in discussing the vibrations of a finite bar, at all events so long as the distance between successive nodes is large compared with $\kappa$. We accordingly take the equation

$$
\frac{\partial^{2} \eta}{\partial t^{2}}+\frac{E \kappa^{2}}{\rho} \frac{\partial^{4} \eta}{\partial x^{4}}=0
$$

as the basis of our subsequent work, together with the formulae

$$
M=E \omega \kappa^{2} \frac{\partial^{2} \eta}{\partial x^{2}}, \quad F=-\frac{\partial M}{\partial x}=-E \omega \kappa^{2} \frac{\partial^{3} \eta}{\partial x^{3}} .
$$

\section{Free-Free Bar.}

To ascertain the normal modes of a finite bar we assume as usual that $\eta$ varies as $\cos (n t+\epsilon)$. The equation (13) of the preceding section then reduces to

$$
\frac{\partial^{4} \eta}{\partial x^{4}}=m^{4} \eta
$$

where

$$
m^{4}=n^{2} \rho / \kappa^{2} E \text {. }
$$

It is to be noted that $m$ is of the nature of the reciprocal of a line. The solution of (1) is

$$
\eta=A \cosh m x+B \sinh m x+C \cos m x+D \sin m x,
$$

the time-factor being for the present omitted. The three ratios $A: B: C: D$, and the admissible values of $m$, and thence of $n^{2}$, are fixed by the four terminal conditions, viz. two for each end. 
Take first the case of a perfectly free bar, of length $l$, say. If we take the origin at the middle*, these conditions are, by $\S 45(14)$,

$$
\eta^{\prime \prime}=0, \quad \eta^{\prime \prime \prime}=0 \quad\left[x= \pm \frac{1}{2} l .\right.
$$

The normal modes fall naturally into two classes; in one of these $\eta$ is an even, in the other an odd function of $x$. For the symmetrical vibrations we have

$$
\eta=A \cosh m x+C \cos m x,
$$

with the terminal conditions

$$
\left.\begin{array}{l}
A \cosh \frac{1}{2} m l-C \cos \frac{1}{2} m l=0, \\
A \sinh \frac{1}{2} m l+C \sin \frac{1}{2} m l=0,
\end{array}\right\}
$$

whence

$$
\tanh \frac{1}{2} m l=-\tan \frac{1}{2} m l \text {. }
$$

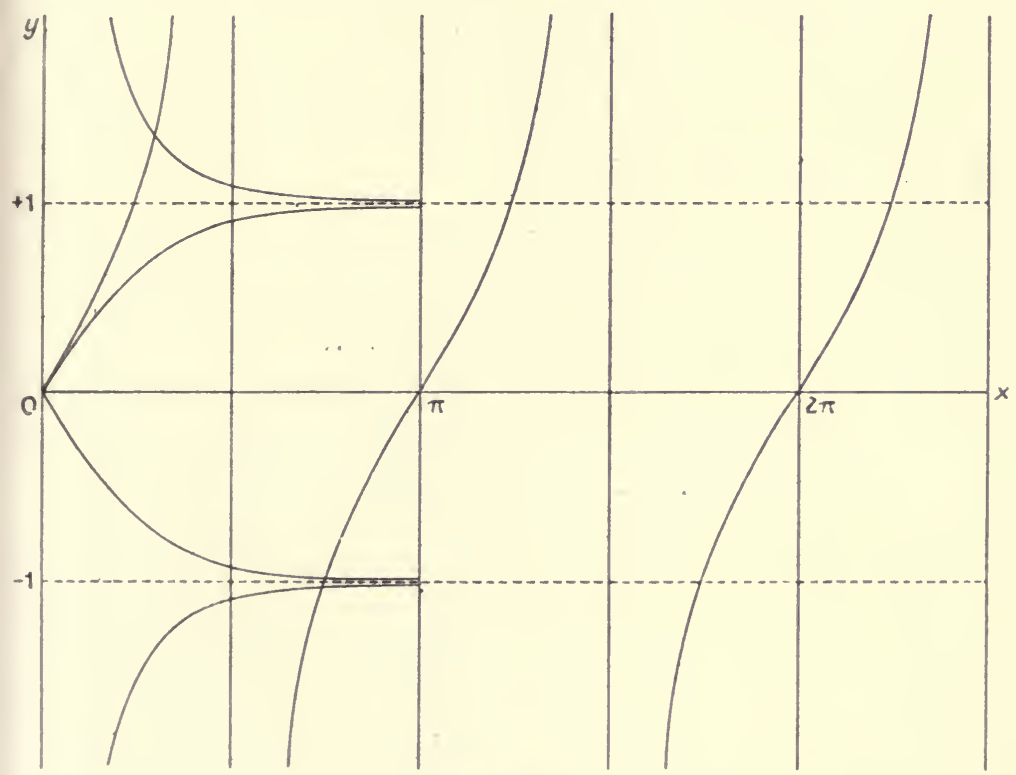

Fig. 44.

The roots of this equation are easily found approximately by graphical construction, viz. as the abscissae of the intersections of the curves $y=\tan x, y=-\tanh x$, the latter of which is

* This improvement on the ordinary procedure is due to Sir A. G. Greenhill, Mess. of Math. vol. xvi., p. 115 (1886). 
asymptotic to the line $y=-1$. The figure shews that we have approximately

$$
\frac{1}{2} m l=\left(s-\frac{1}{4}\right) \pi+\alpha_{s},
$$

where $s=1,2,3, \ldots$, and $\alpha_{8}$ is small. It follows from (2) that the frequencies of the successive normal modes of symmetrical type are approximately proportional to $3^{2}, 7^{2}, 1^{2}, \ldots$ For a more exact computation of the roots we have

$$
\tan \alpha_{8}=\frac{\tan \frac{1}{2} m l+1}{1-\tan \frac{1}{2} m l}=\frac{1-\tanh \frac{1}{2} m l}{1+\tanh \frac{1}{2} m l}=e^{-m l}=\zeta_{8} e^{-2 a_{8}},
$$

where

$$
\zeta_{8}=e^{-28 \pi+\frac{1}{2} \pi} \text {. }
$$

Hence $\quad \alpha_{8}=\tan ^{-1}\left(\zeta_{8} e^{-2 a_{8}}\right)=\zeta_{8} e^{-2 a_{8}}-\frac{1}{3} \zeta_{8}^{3} e^{-6 a_{8}}+\ldots$

Since $\zeta_{s}$ is small, even for $s=1$ (viz. $\zeta_{1}=00898$ ), this is easily solved by successive approximation.

In the asymmetric modes we have

$$
\eta=B \sinh m x+D \sin m x,
$$

with the terminal conditions

$$
\left.\begin{array}{l}
B \sinh \frac{1}{2} m l-D \sin \frac{1}{2} m l=0, \\
B \cosh \frac{1}{2} m l-D \cos \frac{1}{2} m l=0,
\end{array}\right\}
$$

whence $\quad \tanh \frac{1}{2} m l=\tan \frac{1}{2} m l$.

The roots of this are given by the intersections of the curves $y=\tan x, y=\tanh x$, the latter of which is asymptotic to the line $y=1$; see Fig. 44. It appears that

$$
\frac{1}{2} m l=\left(s+\frac{1}{4}\right) \pi-\beta_{s},
$$

where $s=1,2,3, \ldots$, and $\beta_{8}$ is small. The corresponding frequencies are approximately proportional to $5^{2}, 9^{2}, 13^{2}, \ldots$ For the more exact calculation we have

$$
\tan \beta_{s}=\frac{1-\tan \frac{1}{2} m l}{1+\tan \frac{1}{2} m l}=\frac{1-\tanh \frac{1}{2} m l}{1+\tanh \frac{1}{2} m l}=e^{-m l}=\zeta_{s} e^{2 \beta_{s}},
$$

where

$$
\zeta_{8}=e^{-2 s \pi-\frac{1}{2} \pi} \text {. }
$$

Hence

$$
\beta_{8}=\tan ^{-1}\left(\zeta_{8} e^{2 \beta_{8}}\right)=\zeta_{8} e^{2 \beta_{8}}-\frac{1}{3} \zeta_{8}^{3} e^{-6 \beta_{8}}+
$$

Since $\zeta_{1}=\cdot 00039$, the approximation is very rapid, even for $s=1$. 
Combining the results for the two classes it is found that

$$
m l / \pi=1.50562,2.49975,3.50001, \ldots, \quad \ldots(19)
$$

where the values for the symmetric and asymmetric types alternate. The subsequent numbers are adequately represented by $s+\frac{1}{2}$. The fact that the frequencies are approximately proportional to $3^{2}, 5^{2}, 7^{2}, \ldots$ was ascertained, from observation alone, by Chladni*.

To examine the form assumed by the bar in any normal mode, we require the ratio of the arbitrary constants, as determined by (6) or (13). Thus in the case of symmetry we have

$$
\eta=C\left(\cos \frac{1}{2} m l \cosh m x+\cosh \frac{1}{2} m l \cos m x\right) \cos (n t+\epsilon),
$$

where $m$ is a root of (7). The curve may be traced with the help of a table of hyperbolic functions, and the positions of the nodes found by interpolation. The form assumed in the gravest mode is shewn in Fig. 45. The nodes here are at a distance of $\cdot 224$ of the length from the ends.

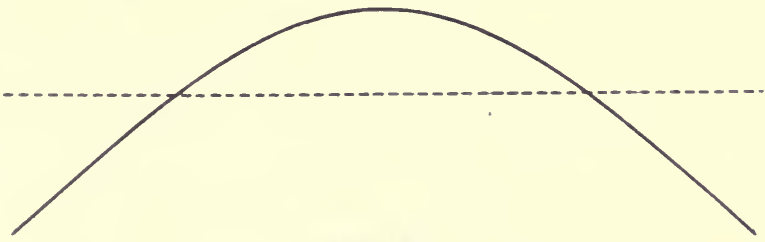

Fig. 45.

The corresponding formula for the asymmetric modes is $\eta=C\left(\sin \frac{1}{2} m l \sinh m x+\sinh \frac{1}{2} m l \sin m x\right) \cos (n t+\epsilon)$, where $m$ is determined by (14).

\section{Clamped-free Bar.}

The next most interesting case is that of a bar clamped at one end and free at the other. Here also there is an advantage in taking the origin at the middle point of the length + . The terminal conditions then are

$$
\eta=0, \eta^{\prime}=0 \quad\left[x=-\frac{1}{2} l\right]
$$

* E. F. F. Chladni, born at Wittenberg 1756, died at Breslau 1827. Distinguished by his experimental researches in acoustics. These are recorded in his book Die Akustik, Leipzig, 1802.

† Greenhill, l.c. 
at the clamped end, and

$$
\eta^{\prime \prime}=0, \quad \eta^{\prime \prime}=0 \quad\left[x=\frac{1}{2} l\right]
$$

for the free end. In one class of vibrations we have

$$
\eta=A \cosh m x+D \sin m x,
$$

with the conditions

$$
\left.\begin{array}{r}
A \cosh \frac{1}{2} m l-D \sin \frac{1}{2} m l=0, \\
-A \sinh \frac{1}{2} m l+D \cos \frac{1}{2} m l=0,
\end{array}\right\}
$$

whence

$$
\operatorname{coth} \frac{1}{2} m l=\tan \frac{1}{2} m l \text {. }
$$

This is solved graphically by the intersections of the curves $y=\tan x, y=\operatorname{coth} x$, the latter of which has $y=1$ as an asymptote; see Fig. 44. We have, approximately,

$$
\frac{1}{2} m l=\left(s+\frac{1}{4}\right) \pi+a_{8}^{\prime},
$$

where $s=0,1,2,3, \ldots$, and $\alpha_{s}^{\prime}$ is small. This leads to

$$
\tan \alpha_{s}^{\prime}=\zeta_{s} e^{-2 a_{s}^{\prime}},
$$

where

$$
\zeta_{8}=e^{-2 s \pi-\frac{1}{2} \pi},
$$

whence

$$
\alpha_{s}^{\prime}=\zeta_{s} e^{-2 a_{s}^{\prime}}-\frac{1}{3} \zeta_{s}^{3} e^{-6 a_{s}^{\prime}}+\ldots,
$$

which can easily be solved by successive approximation, except in the case of the first root $(s=0)$. For this special methods are necessary*. In the remaining type of vibrations we have

$$
\eta=B \sinh m x+C \cos m x,
$$

with

$$
\left.\begin{array}{rl}
-B \sinh \frac{1}{2} m l+C \cos \frac{1}{2} m l & =0, \\
B \cosh \frac{1}{2} m l+C \sin \frac{1}{2} m l & =0,
\end{array}\right\}
$$

whence

$$
\operatorname{coth} \frac{1}{2} m l=-\tan \frac{1}{2} m l \text {. }
$$

The intersections of the curves $y=\tan x, y=-\operatorname{coth} x$ are also shewn in Fig. 44. The roots of (12) are given by

$$
\frac{1}{2} m l=\left(s-\frac{1}{4}\right) \pi-\beta_{8}{ }^{\prime},
$$

where $s=1,2,3, \ldots$ Hence

where

$$
\tan \beta_{8}{ }^{\prime}=\zeta_{s} e^{2 \beta_{8}^{\prime}}
$$

and therefore

$$
\zeta_{8}=e^{-28 \pi+\frac{1}{2} \pi}
$$

$$
\beta_{8}{ }^{\prime}=\zeta_{8} e^{2 \beta_{s}{ }^{\prime}}-\frac{1}{3} \zeta_{8}{ }^{3} e^{6 \beta_{s}{ }^{\prime}}+\ldots
$$

* One such method will be indicated later in connection with the radial vibrations of air in a spherical vessel ( $\$ 84)$. Another very powerful method is explained in Rayleigh's treatise. 
The frequencies of the whole series of normal modes, after the first, are approximately proportional to $3^{2}, 5^{2}, 7^{2}, \ldots$, as found experimentally by Chladni. The accurate solution gives, to five places,

$$
m l / \pi=59686,1 \cdot 49418,2 \cdot 50025, \ldots .
$$

In the modes which follow the first we have respectively one, two, three, ... internal nodes. The annexed figure shews the gravest mode.

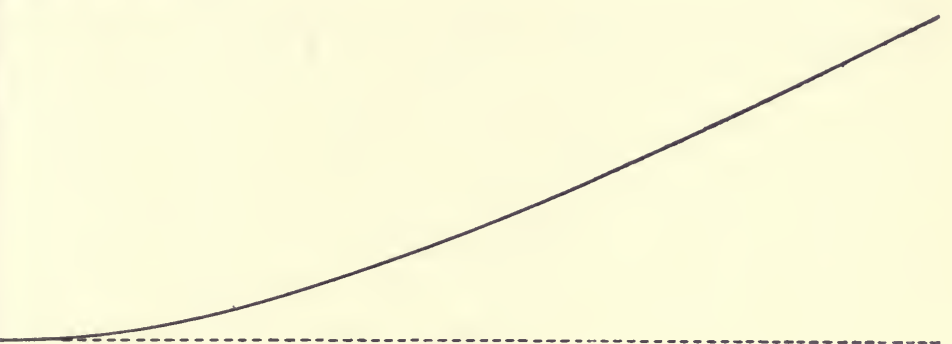

Fig. 46.

Other problems, which are however of less interest, may be obtained by varying the terminal conditions. We will only notice the case where both ends are "supported," i.e. fixed in position but free from terminal couples. The conditions then are, by $\S 45(14)$,

$$
\eta=0, \eta^{\prime \prime}=0 \quad\left[x= \pm \frac{1}{2} l\right] .
$$

In the symmetrical class we have

$$
\eta=C \cos m x \cdot \cos (n t+\epsilon),
$$

with $\cos \frac{1}{2} m l=0$, whence

$$
m l / \pi=1,3,5, \ldots .
$$

In the asymmetric class

$$
\begin{gathered}
\eta=C \sin m x \cdot \cos (n t+\epsilon), \\
m l / \pi=2,4,6, \ldots .
\end{gathered}
$$

The frequencies are, by $\S 46$ (2), proportional to the values of $m^{2}$, and so to the squares of the natural numbers.

The foundations of the theory of the transverse vibrations were laid by D. Bernoulli (1735) and Euler (1740). The latter also gave the numerical solution of the period equation in a few of the more important cases. In more recent times 
the calculations, including the determination of the nodes \&c., have been greatly extended by Lissajous (1850), Seebeck* (1848) and Lord Rayleigh.

\section{Summary of Results. Forced Vibrations.}

In any one of the preceding cases, and in any particular mode, $m$ varies inversely as $l$, and therefore, by $\$ 46(2)$, the period $2 \pi / n$ will for bars of the same material vary as $l^{2} / \kappa$. Hence for bars which are in all respects similar to one another (geometrically) the period will vary as the linear scale. For bars of the same section the period is as the square of the length. As regards the shape and size of the cross-section, everything depends on the radius of gyration $\kappa$; thus for bars of rectangular section the frequency varies as the thickness in the plane of vibration, and is independent of the lateral dimension. This latter statement needs, however, some qualification; it is implied that the breadth is small compared with the length of the bar, or (more precisely) with the distance between consecutive nodes. When this condition is violated the problem comes under the more complex theory of plates $(\$ 55)$.

It is of interest to compare the frequencies of transverse and longitudinal vibration of a bar in corresponding cases. For a bar free at both ends we have, in the gravest transverse mode,

$$
n^{2}=\frac{\kappa^{2} E}{\rho l^{4}}(m l)^{4}=\frac{\pi^{4} \kappa^{2} E}{\rho l^{4}} \times(1 \cdot 50562)^{4},
$$

whilst in the gravest longitudinal mode

$$
n^{\prime 2}=\frac{\pi^{2} E}{\rho l^{2}}
$$

Hence

$$
\frac{n}{n^{\prime}}=7 \cdot 122 \frac{\kappa}{l}
$$

This explains the relative slowness of the transversal modes. The comparison is due to Poisson.

We pass over the question of determining the motion consequent on arbitrary initial conditions, by means of the normal functions. In the case of the free-free bar, for example, these are given by the expressions in brackets in equations (20) and (21) of $\S 46$.

\footnotetext{
* L, F. W. A, Seebeck (1805-49), professor of physics at Leipzig.
} 
The theory of forced vibrations again, is of little acoustical interest, although it has some technical importance. A simple example is furnished by the coupling rod which connects the wheels of a locomotive. Attending only to the vertical component of the motion, and treating the bar as uniform, we have to solve the equation (13) of $\S 45$ subject to the conditions

$$
\eta=\beta \cos (p t+\alpha), \eta^{\prime \prime}=0 \quad\left[x= \pm \frac{1}{2} l\right],
$$

where $n$ is the angular velocity of the wheels, and $\beta$ is the vertical amplitude. The forced oscillation is evidently of symmetrical type, and we therefore assume

$$
\eta=(A \cosh m x+C \cos m x) \cos (p t+\alpha) .
$$

This satisfies the differential equation, provided

$$
m^{4}=p^{2} \rho / \kappa^{2} E
$$

whilst the terminal conditions give

$$
\left.\begin{array}{l}
A \cosh \frac{1}{2} m l+C \cos \frac{1}{2} m l=\beta, \\
A \cosh \frac{1}{2} m l-C \cos \frac{1}{2} m l=0,
\end{array}\right\}
$$

the latter equation expressing the absence of terminal couples $\left(\eta^{\prime \prime}=0\right)$. Hence

$$
A=\frac{\beta}{2 \cosh \frac{1}{2} m l}, \quad C=\frac{\beta}{2 \cos \frac{1}{2} m l} .
$$

The oscillations would become dangerously large if $\cos \frac{1}{2} m l$ were small, i.e. if the imposed frequency $(p / 2 \pi)$ were to approximate to that of one of the symmetrical free modes of the bar when "supported" at the ends ( $\$ 47(20)$.

\section{Applications.}

The use of transverse vibrations of bars in music is restricted by the fact that the overtones are not harmonic to the fundamental. If a flat bar, otherwise free, be supported at the nodes of the fundamental (Fig. 45), and struck with a soft hammer, the production of overtones is, however, in some measure discouraged, and musical instruments of a kind (such as the "glass harmonica") have been constructed on this plan.

The most important application is in the tuning fork. 
Theory and observation alike shew that the effect of curving a bar is to lower the pitch of the gravest mode and to make the nodes approach the centre. It was found by Chladni that when the bar takes the form of an elongated $U$, the nodes are very close to the bend. The amplitude of vibration at the centre of the bend will therefore be small compared with that at the end of the prongs. The circumstances are somewhat modified by the attachment of the stem, but the transmission of energy is comparatively slow, and the vibrations have considerable persistence. A fork may also be compared to a couple of bars each clamped at one end, and the formula (2) of $\S 46$, with $m l / \pi=59686$, may be used to estimate the frequency theoretically. If this analogy were exact there would of course be no loss of energy of the kind just referred to.

Massive forks are usually set into vibration by means of a violoncello bow applied to one prong near the free end. The production of overtones having nodes in this neighbourhood is thus discouraged. The fundamental is further reinforced relatively to the other modes if the stem be screwed into the upper face of a resonance box of suitable dimensions.

When a fork is excited in this or in other ways, it often happens that the motion is not in the first instance symmetrical with respect to the medial plane. In that event the vibration may be regarded as made up of a symmetrical and an unsymmetrical component. These will in general have slightly different frequencies, and beats may be produced. But unless the stem be very firmly fixed the vibrations of the latter class are rapidly dissipated by being communicated to the support, since they involve an oscillation of the centre of mass of the fork.

The first overtone of a fork may be elicited in considerable intensity by bowing one of the prongs near the bend; the note produced is very shrill.

\section{Effect of Permanent Tension.}

In the theory developed in $\S 45$ it was assumed that the longitudinal tension, when integrated over the area of the cross-section, vanishes. It is easy to see that the effect of 
a permanent tension $P$ is merely to add a term $P \eta^{\prime \prime}$ to the equation (13) of $\S 45$, so that

$$
\frac{\partial^{2} \eta}{\partial t^{2}}=c_{0}^{2} \frac{\partial^{2} \eta}{\partial x^{2}}-\frac{\kappa^{2} E}{\rho} \frac{\partial^{4} \eta}{\partial x^{4}}
$$

where

$$
c_{0}^{2}=P / \rho \omega \text {. }
$$

This equation has been employed to estimate the effect of stiffness of a piano-wire on the sequence of proper tones, but the matter is complicated by the uncertainty as to the nature of the terminal conditions. A wire, where it passes over a bridge, cannot be quite accurately regarded either as merely "supported" or as "clamped." The question will perhaps be sufficiently illustrated if we consider a wave-system

$$
\eta=C \cos k(c t-x)
$$

on an unlimited wire. We find, on substitution in (1)

$$
c^{2}=c_{0}^{2}+c_{1}^{2}
$$

where

$$
c_{1}^{2}=\frac{E}{\rho} \cdot k^{2} \kappa^{2},
$$

i.e. $c_{1}$ is the velocity of transverse waves of length $2 \pi / k$ on a bar free from tension. We have seen that in the case of a pianostring $E / \rho$ is large compared with $c_{0}{ }^{2}$; on the other hand $\kappa$ is usually an exceedingly minute fraction of the wave-length. In the graver modes of a piano-string this second influence predominates, and $\left(c_{1} / c_{0}\right)^{2}$ is small ; the wave-velocity is practically unaffected by stiffness, and the harmonic sequence is not disturbed. It is only in the case of the modes of very high order, where the length is divided into a large number of vibrating segments, that a sensible effect could be looked for. It has already been stated that in the pianoforte such modes are, so far as may be, discouraged on independent grounds. In any case it appears from (4) that the effect of stiffness is relatively less important, the greater the value of $c_{0}$, i.e. the tighter the wires are strung.

\section{Vibrations of a Ring. Flexural and Extensional} Modes.

The theory of the vibrations of a circular ring is important as throwing light on some later questions which can only be 
dealt with imperfectly in this book, owing to the difficulties of an exact investigation. As various points of interest arise, we treat the matter somewhat fully.

The ring is supposed to be uniform, and the section is assumed to be symmetrical with respect to a plane perpendicular to the axis. We further consider only vibrations parallel to this plane. Let $u, v$ be the displacements of an element of the ring along and at right angles to the original radius vector, so that the polar coordinates of the element are changed from $(a, \theta)$ to $(a+u, \theta+v / a)$. We require expressions for the extension, and for the change of curvature. In consequence of the assumed smallness of the displacements, we may calculate the instalments of these quantities which are due to $u$ and $v$ separately, and add the results. The radial displacement by itself changes the length of an element from $a \delta \theta$ to $(a+u) \delta \theta$, and so causes an extension $u / a$. The transverse displacement obviously contributes $\partial v / a \partial \theta$. The total extension is therefore

$$
\frac{1}{a}\left(u+\frac{\partial v}{\partial \theta}\right)
$$

Again, in consequence of the radial displacement alone the normal to the curve is rotated backwards so as to make an angle $\partial u / a \partial \theta$ with the radius, and the mutual inclination of the normals at the ends of an element $a \delta \theta$ is accordingly diminished by $\partial^{2} u / a \partial \theta^{2} \cdot \delta \theta$. Dividing the angle between the normals by the altered length $(a+u) \delta \theta$ we get the altered curvature, thus

$$
\left(\delta \theta-\frac{\partial^{2} u}{a \partial \theta^{2}} \delta \theta\right) \div(a+u) \delta \theta=\frac{1}{a}-\frac{1}{a^{2}}\left(\frac{\partial^{2} u}{\partial \theta^{2}}+u\right) \text {. }
$$

Since the transverse displacement $v$ by itself contributes nothing, the increase of curvature is

$$
-\frac{1}{a^{2}}\left(\frac{\partial^{2} u}{\partial \theta^{2}}+u\right)
$$

The resultant stress across any section may be resolved into a radial shearing force $P$, a tangential tension $Q$, and a bending moment $M$. On the principles of $\$ 43,45$ we have

$$
Q=\frac{E \omega}{a}\left(u+\frac{\partial v}{\partial \theta}\right), \quad M=-\frac{E \omega \kappa^{2}}{a^{2}}\left(\frac{\partial^{2} u}{\partial \theta^{2}}+u\right),
$$


the bending moment being now proportional to the increase of curvature.

Resolving along and perpendicular to the radius vector the forces on a mass-element $\rho \omega a \delta \theta$, we have (see Fig. 47)

$$
\rho \omega a \delta \theta . \ddot{u}=\delta P-Q \delta \theta, \quad \rho \omega a \delta \theta . \ddot{v}=\delta Q+P \delta \theta ;
$$

and, taking moments about a normal to the plane of the ring,

$$
\delta M-P a \delta \theta=0,
$$

the rotational inertia being neglected as in the case of a straight bar $(§ 45)$. Thus

$$
\begin{array}{r}
\rho \omega a \frac{\partial^{2} u}{\partial t^{2}}=\frac{\partial P}{\partial \theta}-Q, \quad \rho \omega a \frac{\partial^{2} v}{\partial t^{2}}=\frac{\partial Q}{\partial \theta}+P, \\
\frac{\partial M}{\partial \theta}=P a . \quad \ldots \ldots \ldots \ldots \ldots . . .
\end{array}
$$

and

These, together with (3), are the equations of our problem. It is easily seen that they cannot be satisfied on the assumption that the tension $Q$ vanishes, and that accordingly some degree of extension is involved in any mode of vibration. This is readily accounted for, a stress of this kind being necessarily called into play by the inertia of the different portions swinging

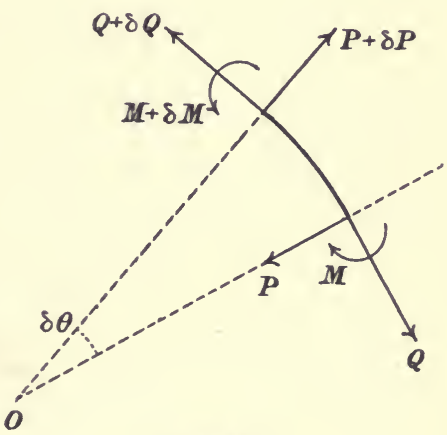

Fig. 47 . in opposite directions. It may be shewn however that in the "flexural" modes to be referred to presently the corresponding strains are small compared with those involved in the change of curvature.

Eliminating $P, Q, M$ between (3), (4), and (5), we find

$$
\left.\begin{array}{l}
\frac{\partial^{2} u}{\partial t^{2}}+\frac{E}{\rho a^{2}}\left\{u+\frac{\partial v}{\partial \theta}+\frac{\kappa^{2}}{a^{2}}\left(\frac{\partial^{2} u}{\partial \theta^{2}}+\frac{\partial^{4} u}{\partial \theta^{4}}\right)\right\}=0, \\
\frac{\partial^{2} v}{\partial t^{2}}-\frac{E}{\rho a^{2}}\left\{\frac{\partial u}{\partial \theta}+\frac{\partial^{2} v}{\partial \theta^{2}}-\frac{\kappa^{2}}{a^{2}}\left(\frac{\partial u}{\partial \theta}+\frac{\partial^{3} u}{\partial \theta^{3}}\right)\right\}=0 .
\end{array}\right\}
$$

To ascertain the normal modes we assume that $u$ and $v$ vary as $\cos (n t+\epsilon)$. Again, the ring being complete, $u$ and $v$ are 
necessarily periodic functions of $\theta$, the period being $2 \pi$, and can accordingly be expanded by Fourier's theorem. in series of sines and cosines of multiples of $\theta$; moreover it is easily proved that the terms of any given rank in the expansion must satisfy the equations separately. We find, in fact, that a sufficient assumption for our purpose is

$$
u=A \cos s \theta \cdot \cos (n t+\epsilon), \quad v=B \sin s \theta \cdot \cos (n t+\epsilon),
$$

where $s$ is integral or zero. This leads to

$$
\begin{array}{r}
\left\{\beta-1-s^{2}\left(s^{2}-1\right) \frac{\kappa^{2}}{a^{2}}\right\} A-s B=0, \\
-\left\{s+s\left(s^{2}-1\right) \frac{\kappa^{2}}{a^{2}}\right\} A+\left(\beta-s^{2}\right) B=0, \\
\beta=n^{2} a^{2} \rho / E . \ldots \ldots \ldots \ldots \ldots
\end{array}
$$

where

Hence

$$
\beta^{2}-\left\{s^{2}+1+s^{2}\left(s^{2}-1\right) \frac{\kappa^{2}}{a^{2}}\right\} \beta+s^{2}\left(s^{2}-1\right)^{2} \frac{\kappa^{2}}{a^{2}}=0 .
$$

Since $\kappa / a$ is small, the sum of the roots of this quadratic in $\beta$ is $s^{2}+1$, approximately, whilst the product $s^{2}\left(s^{2}-1\right)^{2} \kappa^{2} / a^{2}$ is small. The two roots are therefore

$$
\beta=s^{2}+1, \quad \beta=\frac{s^{2}\left(s^{2}-1\right)^{2}}{s^{2}+1} \frac{\kappa^{2}}{a^{2}},
$$

approximately.

On reference to (8) we see that the former root makes $B=s A$ nearly. The corresponding modes are closely analogous to the longitudinal modes of a straight bar, the potential energy being mainly due to the extension; and the frequencies, which are given by

$$
n^{2}=\left(s^{2}+1\right) \frac{E}{\rho a^{2}},
$$

are, for similar dimensions, of the like order of magnitude. The case $s=0$ is that of purely radial vibrations.

The vibrations corresponding to the second root are more important. We then have, from (8), $A+s B=0$, nearly; thus

$$
u=A \cos s \theta \cdot \cos (n t+\epsilon), \quad v=-\frac{A}{s} \sin s \theta \cdot \cos (n t+\epsilon),
$$

with

$$
n^{2}=\frac{s^{2}\left(s^{2}-1\right)^{2}}{s^{2}+1} \frac{E \kappa^{2}}{\rho a^{4}} .
$$


It follows from (1) that the extension is negligible, and the energy mainly flexural. The frequencies are in fact comparable with those of transverse vibration of a bar. In the mode of order $s$ there are $2 s$ nodes, or places of vanishing radial motion, but these are not points of rest, the tangential motion being there a maximum*. In the case $s=1$ the circle is merely displaced as a whole, without deformation, and the period is
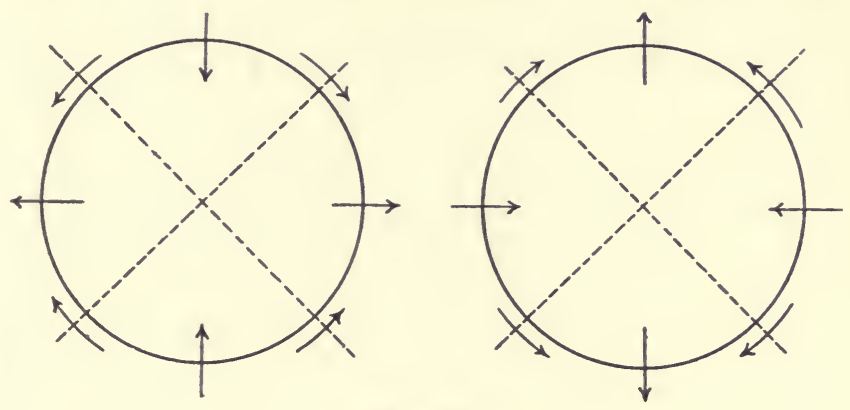

Fig. 48.

accordingly infinite. The most important case is that of $s=2$, where the ring oscillates between two slightly elliptical extreme forms. The arrows in the annexed figure shew the directions of motion at various parts of the circumference at two epochs, separated by half a period, when the ring passes through its equilibrium position. The dotted lines pass through the nodes of the radial vibration.

One further point is to be noticed. Owing to the assumed uniformity of the ring the origin of $\theta$ is arbitrary, and other modes, with the same frequencies, are obtained by adding a constant to $\theta$. In particular we have the flexural mode

$$
u=A \sin s \theta \cdot \cos (n t+\epsilon), \quad v=\frac{A}{s} \cos s \theta \cdot \cos (n t+\epsilon),
$$

with the same value of $n^{2}$ as in (14). We have here an instance of the kind referred to in $\S 16$, where two distinct normal modes

* This point is illustrated by the vibrations of a finger-bowl when excited by drawing a wetted finger along the edge. The point of rubbing is a node as regards the radial vibration, and the crispations on the contained water are accordingly most conspicuous at distances of $45^{\circ}$ on either side, where the radial motion is a maximum. 
have the same frequency, and the modes themselves accordingly become to some extent indeterminate. The case would be altered at once if the ring were not quite uniform, e.g. if it were slightly thicker at one point. The normal modes in which there is a node or a loop respectively, of radial vibration, at this point would differ somewhat in character, and have slightly different frequencies. Accordingly when both modes are excited we should have beats between the corresponding tones. This is a phenomenon often noticeable in the case of bells (and finger-bowls), the inequality being due to a slight defect of symmetry.

The vibrations of a ring in its own plane were first investigated by R. Hoppe (1871); a simplified treatment of the flexural modes was subsequently given by Lord Rayleigh. The theory of vibrations normal to the plane is more intricate, since torsion is involved as well as flexure. The problem has been solved by J. H. Michell (1889), who finds, in the case of circular crosssection,

$$
n^{2}=\frac{s^{2}\left(s^{2}-1\right)^{2}}{s^{2}+1+\sigma} \cdot \frac{E \kappa^{2}}{\rho a^{4}},
$$

where $\sigma$ is Poisson's ratio. 


\section{CHAPTER V}

\section{MEMBRANES AND PLATES}

\section{Equation of Motion of a Membrane. Energy.}

The vibrations of membranes are not very important in themselves, and the conditions assumed for the sake of mathematical simplicity are, moreover, not easily realized experimentally. The theory is however, for a two-dimensional system, comparatively simple, and the results help us to understand in a general way the character of the normal modes in other cases where the difficulties of calculation are much greater, and indeed often insuperable.

The ideal membrane of theory is a material surface such that the stress across any line-element drawn on it is always in the tangent plane. We shall consider only cases where the surface in its undisturbed state is plane, and is in a state of uniform, or "homogeneous," stress; i.e. it is assumed that the stresses across any two parallel and equal lines are the same in direction and magnitude. We further suppose, for simplicity, that the stress across any line-element is perpendicular to that element. It follows, exactly as in hydrostatics, from a consideration of the forces acting on the contour of a triangular area, that the stress (per unit length) is the same for all directions of a line-element. This uniform stress is called the "tension" of the membrane; we denote it by $P$. Its dimensions are those of a force divided by a line, or $\left[M T^{-2}\right]$.

We take rectangular axes of $x, y$ in the plane of the undisturbed membrane, and denote by $\zeta$ the displacement normal to this plane. The surface-density (i.e. the mass per 
unit area) is assumed to be uniform, and is denoted by $\rho$. To form the equations of motion we calculate the forces on the sides of a rectangular element $\delta x \delta y$ having its centre at $(x, y)$. In the displaced position, the gradient of a line parallel to $x$ is $\partial \zeta / \partial x$, and that of a line parallel to $y$ is $\partial \zeta / \partial y$. Hence the stress across a line through the centre of the element parallel to $\delta y$, when resolved in the direction of the normal to the plane $x y$, is $P \partial \zeta / \partial x . \delta y$. The corresponding components of force on the two edges $\delta y$ of the rectangle are

$$
\pm P\left\{\frac{\partial \zeta}{\partial x} \pm \frac{\partial}{\partial x}\left(\frac{\partial \zeta}{\partial x}\right) \cdot \frac{1}{2} \delta x\right\} \delta y
$$

where the upper signs relate to the edge whose abscissa is $x+\frac{1}{2} \delta x$, and the lower to the edge $x-\frac{1}{2} \delta x$. The sum of these gives $P \partial^{2} \zeta / \partial x^{2} . \delta x \delta y$. A similar calculation for the two edges $\delta x$ gives $P \partial^{2} \zeta / \partial y^{2} . \delta x \delta y$. The resultant force on the rectangle is therefore

$$
P\left(\frac{\partial^{2} \zeta}{\partial x^{2}}+\frac{\partial^{2} \zeta}{\partial y^{2}}\right) \delta x \delta y
$$

The above may be compared with the investigation by which, in the theory of Capillarity, the tensions across the boundary of an element $\delta S^{\top}$ of a soap-film are shewn to be equivalent to a normal force

$$
P\left(\frac{1}{R_{1}}+\frac{1}{R_{2}}\right) \delta S,
$$

where $R_{1}, R_{2}$ are the principal radii of curvature of the surface. It is shewn in books on solid geometry that, if $\zeta$ denote distance from the plane $x y$, we have

$$
\frac{1}{R_{1}}+\frac{1}{R_{2}}=\frac{\partial^{2} \zeta}{\partial x^{2}}+\frac{\partial^{2} \zeta}{\partial y^{2}}
$$

at points where the inclination of the tangent plane to $x y$ is small.

Equating the expression (1) to the acceleration of momentum of the element, viz. $\rho \delta x \delta y . \ddot{\zeta}$, we obtain the equation of motion

$$
\rho \frac{\partial^{2} \zeta}{\partial t^{2}}=P\left(\frac{\partial^{2} \zeta}{\partial x^{2}}+\frac{\partial^{2} \zeta}{\partial y^{2}}\right) .
$$

This is due to Euler (1766). 
The kinetic energy is given by

$$
T=\frac{1}{2} \rho \iint\left(\frac{\partial \zeta}{d t}\right)^{2} d x d y
$$

taken over the area of the membrane.

The potential energy is found most easily as the work required to stretch the membrane. As in the theory of capillarity this is equal to the tension $P$ multiplied by the increase of area. Now if a prism be constructed on a rectangular element $\delta x \delta y$ of the plane $x y$ as base, this will cut out from the displaced membrane a nearly rectangular portion whose sides are

$$
\sqrt{ }\left\{1+\left(\frac{\partial \zeta}{\partial x}\right)^{2}\right\} \delta x, \quad \sqrt{ }\left\{1+\left(\frac{\partial \zeta}{\partial y}\right)^{2}\right\} \delta y
$$

and whose area is therefore, to the second order,

$$
\begin{gathered}
\left\{1+\frac{1}{2}\left(\frac{\partial \zeta}{\partial x}\right)^{2}+\frac{1}{2}\left(\frac{\partial \zeta}{\partial y}\right)^{2}\right\} \delta x \delta y . \\
V=\frac{1}{2} P \iint\left\{\left(\frac{\partial \zeta}{\partial x}\right)^{2}+\left(\frac{\partial \zeta}{\partial y}\right)^{2}\right\} d x d y .
\end{gathered}
$$

Hence

The same expression is obtained by calculating, from the expression (1), the work done by normal pressures applied (as in $\$ 22$ ) to deform the membrane into its actual shape, the ratio of $\zeta$ to its final value being, at any stage of the process, the same all over the membrane. The result is

$$
V=-\frac{1}{2} P \iint\left(\frac{\partial^{2} \zeta}{\partial x^{2}}+\frac{\partial^{2} \zeta}{\partial y^{2}}\right) \zeta d x d \bar{y}
$$

The reader who is familiar with the theory of attractions will recognize that this is equal to

$$
V=\frac{1}{2} P \int \zeta \frac{\partial \zeta}{\partial n} d s+\frac{1}{2} P \iint\left\{\left(\frac{\partial \zeta}{\partial x}\right)^{2}+\left(\frac{\partial \zeta}{\partial y}\right)^{2}\right\} d x d y
$$

where in the first term the integration extends over all the elements $\delta s$ of the contour, and $\delta n$ is an element of the normal to $\delta s$ drawn inwards, in the plane of the membrane. Since at a fixed edge $\zeta=0$, the formula agrees with (5). 


\section{Square Membrane. Normal Modes.}

To ascertain the normal modes of a limited membrane we assume as usual that $\zeta$ varies as $\cos (n t+\epsilon)$, so that

$$
\frac{\partial^{2} \zeta}{\partial x^{2}}+\frac{\partial^{2} \zeta}{\partial y^{2}}+k^{2} \zeta=0
$$

where

$$
k^{2}=n^{2} \rho / P \text {. }
$$

At a fixed boundary we must have $\zeta=0$. It is found that the solution of (1) subject to this condition is possible only for a series of definite values of $k$, which determine, by (2), the corresponding frequencies.

In the case of a rectangular membrane, we take the origin at a corner, and the axes of $x, y$ along the edges which meet there. The equations of the remaining edges being, say, $x=a, y=b$, the equation (1) and the boundary condition are satisfied by

$$
\zeta=C \sin \frac{s \pi x}{a} \sin \frac{s^{\prime} \pi y}{b} \cos (n t+\epsilon),
$$

where $s, s^{\prime}$ are integers, provided

$$
k^{2}=\pi^{2}\left(\frac{s^{2}}{a^{2}}+\frac{s^{2}}{b^{2}}\right)
$$

It may be shewn, by an easy extension of Fourier's theorem, that (3) is the only admissible type of solution in the present case; it was given by Poisson in 1829 .

In any normal mode for which $s$ or $s^{\prime}>1$, we have nodal lines parallel to the edges. It appears from (4) that if the ratio $a^{2}: b^{2}$ is not equal to that of two integers, the frequencies are all distinct, and the nodal lines are restricted to these forms. But if $a^{2}: b^{2}$ is commensurable, some of the periods coincide, and the corresponding modes may be superposed in arbitrary proportions $(\$ 16)$. The nodal lines may then assume a great variety of forms. The simplest instance is that of the square membrane $(a=b)$, when

$$
k^{2}=\frac{\pi^{2}}{a^{2}}\left(s^{2}+s^{\prime 2}\right)
$$


Thus by superposition of the modes for which $s=2, s^{\prime}=1$ and $s=1, s^{\prime}=2$, respectively, we get

$$
\begin{gathered}
\zeta \propto \sin \frac{2 \pi x}{a} \sin \frac{\pi y}{a}+\lambda \sin \frac{\pi x}{a} \sin \frac{2 \pi y}{a} \\
\propto \sin \frac{\pi x}{a} \sin \frac{\pi y}{a}\left(\cos \frac{\pi x}{a}+\lambda \cos \frac{\pi y}{a}\right),
\end{gathered}
$$

where $\lambda$ may have any value. For example, in the cases $\lambda= \pm 1$ the diagonals $x+y=a, x-y=0$, respectively, are nodal lines. The figure shews the cases $\lambda=0, \lambda=-\frac{1}{2}, \lambda=-1$, which give a sufficient indication of the various forms that may arise.
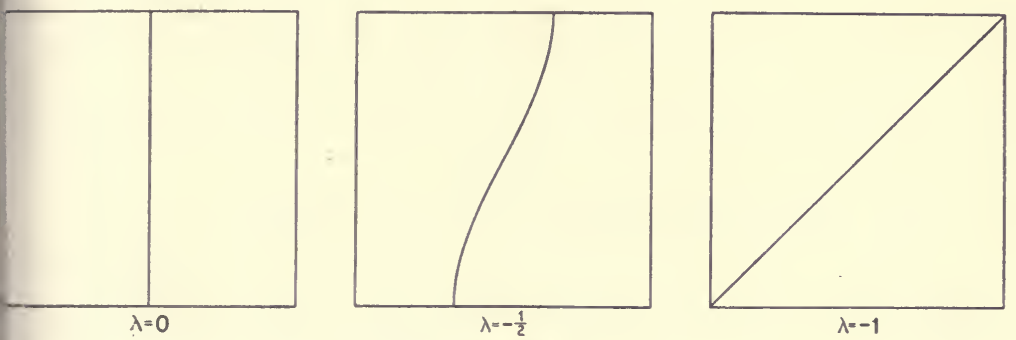

Fig. 49 .

Again, by superposition of the cases $s=3, s^{\prime}=1$ and $s=1$, $s^{\prime}=3$, we get

$$
\begin{aligned}
& \zeta \propto \sin \frac{3 \pi x}{a} \sin \frac{\pi y}{a}+\lambda \sin \frac{\pi x}{a} \sin \frac{3 \pi y}{a} \\
& \propto \sin \frac{\pi x}{a} \sin \frac{\pi y}{a}\left\{3-4 \sin ^{2} \frac{\pi x}{a}+\lambda\left(3-4 \sin ^{2} \frac{\pi y}{a}\right)\right\} .
\end{aligned}
$$

The cases $\lambda=0, \lambda= \pm \frac{1}{3}, \lambda= \pm 1$ are shewn in Fig. 50; intermediate forms are readily supplied in imagination.

A still greater variety is introduced by the fact that a number which is the sum of two squares can sometimes be so resolved in more than one way. For example, the modes for which

$$
\left.\begin{array}{rl}
s & =4,7,1,8, \\
s^{\prime} & =7,4,8,1,
\end{array}\right\}
$$

respectively, have all the same frequency. 

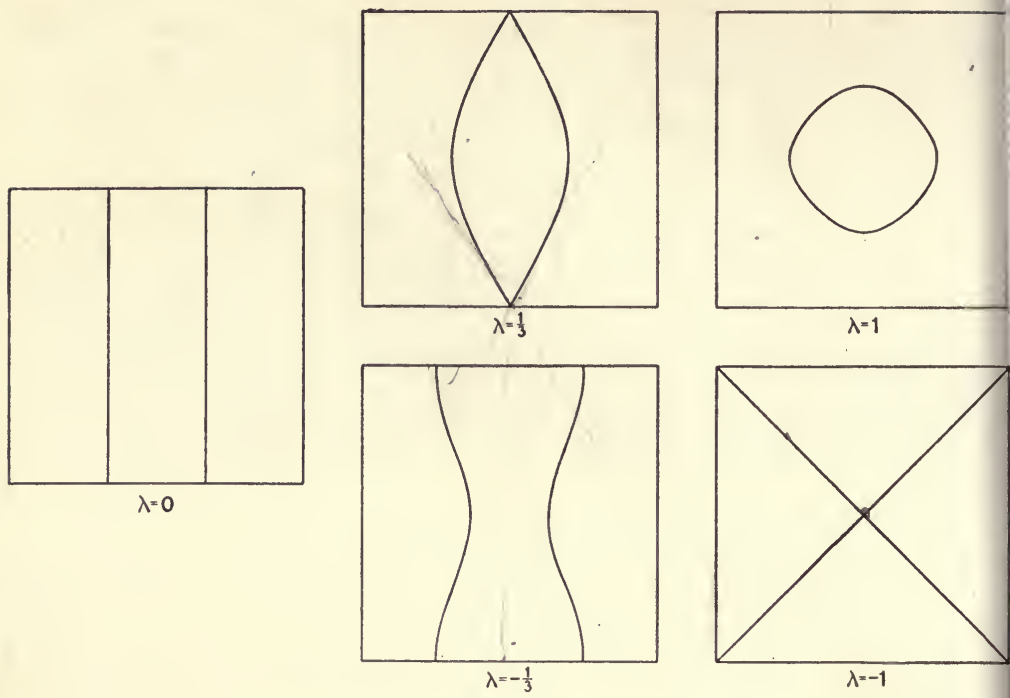

Fig. 50 .

\section{Circular Membrane. Normal Modes.}

In the case of the circular membrane we naturally have recourse to polar coordinates, with the origin at the centre. The differential equation may be obtained by transformation of $\$ 52(3)$, but a more direct process is preferable.

Take first the case of the symmetrical vibrations where $\zeta$ is a function of $r$, the distance from $O$, only. The stress across a circle of radius $r$ has a resultant $P .2 \pi r . \partial \zeta / \partial r$ normal to the plane of the undisturbed membrane, and the difference of the stresses on the edges of the annulus whose inner and outer radii are $r$ and $r+\delta r$ gives a force

$$
\frac{\partial}{\partial r}\left(P \cdot 2 \pi r \cdot \frac{\partial \zeta}{\partial r}\right) \delta r
$$

Equating this to $\rho .2 \pi r \delta r . \ddot{\zeta}$, which is the acceleration of momentum of the annulus, we get

$$
\rho \frac{\partial^{2} \zeta}{\partial t^{2}}=\frac{P}{r} \frac{\partial}{\partial r}\left(r \frac{\partial \zeta}{\partial r}\right)
$$

If $\zeta$ varies as $\cos (n t+\epsilon)$, this reduces to

$$
\frac{\partial^{2} \zeta}{\partial r^{2}}+\frac{1}{r} \frac{\partial \zeta}{\partial r}+k^{2} \zeta=0
$$

where $k^{2}=n^{2} \rho / P$, as before. 
If we assume, as is necessarily the case when the origin is included within the region to which (2) applies, that $\zeta$ can be expanded in a series of ascending powers of $r$, the coefficients (aftèr the first) may be found by substitution in (2), and we obtain

$$
\zeta=C J_{0}(k r) \cdot \cos (n t+\epsilon),
$$

provided

$$
J_{0}(z)=1-\frac{z^{2}}{2^{2}}+\frac{z^{4}}{2^{2} \cdot 4^{2}}-\ldots
$$

This is the Bessel's Function * of zero order, "of the first kind," which we have already met with in $\$ 31$; it is represented graphically in Fig. 51. If $a$ be the radius of the boundary,

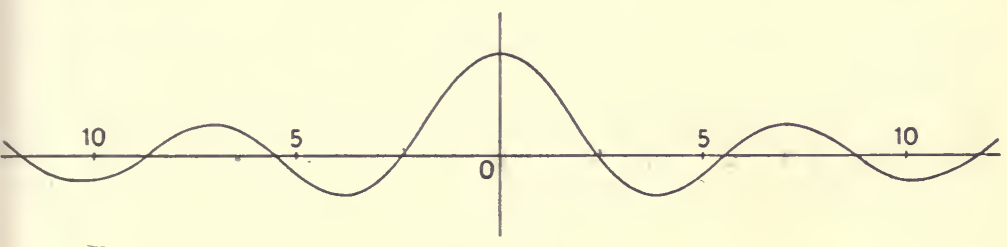

Fig. 51 .

supposed fixed, the admissible values of $k$ and thence of $n$ are determined by the equation

$$
J_{0}(k a)=0,
$$

viz. we have

$$
k a / \pi=\cdot 7655,1 \cdot 7571,2 \cdot 7546,3 \cdot 7534, \ldots
$$

the numbers tending to the form $m-\frac{1}{4}$, where $m$ is integral. The first of these roots corresponds to the gravest of all the normal modes of the membrane. In the $m$ th mode there are $m-1$ nodal circles, in addition to the edge, whose radii are given by the roots of lower order. Thus in the case of the second root we have for the nodal circle $k r / \pi=\cdot 7655$, whence $r / a=4356$. The characters of the various normal modes will be understood from Fig. 51, which may be taken to represent a section through the centre, normal to the plane of the membrane.

* F. W. Bessel (1784-1846), director of the observatory at Königsberg $1810-46$. 
The complete solution of the differential equation (2), which is of the second order, would consist of the sum of two definite functions of $k r$, each multiplied by an arbitrary constant; but the second solution, which is called a Bessel's Function "of the second kind," becomes infinite for $r=0$, and is therefore inapplicable to a complete circular area. In the case of an annular membrane, however, bounded by concentric circles, both solutions would be admissible, and both would be required in order to satisfy the conditions at the two edges*'.

The theory of the symmetrical vibrations of a circular membrane was given by Poisson (1829), who also calculated approximately a few of the roots of the period-equation (5).

When the vibrations are not symmetrical we may begin by calculating the forces on a quasi-rectangular element of area bounded by two radii vectores and two concentric circles, the sides being accordingly $\delta r$ and $r \delta \theta$. The stresses on the curved sides give a resultant

$$
\frac{\partial}{\partial r}\left(\operatorname{Pr} \delta \theta \cdot \frac{\partial \zeta}{\partial r}\right) \delta r
$$

normal to the plane, whilst the stresses on the straight sides produce

$$
\frac{\partial}{\partial \theta}\left(P \delta r \cdot \frac{\partial \zeta}{r \partial \theta}\right) \delta \theta
$$

Equating the sum of these expressions to $\rho r \delta \theta \delta r . \ddot{\zeta}$, we obtain

$$
\rho \frac{\partial^{2} \zeta}{\partial t^{2}}=P\left\{\frac{1}{r} \frac{\partial}{\partial r}\left(r \frac{\partial \zeta}{\partial r}\right)+\frac{1}{r^{2}} \frac{\partial^{2} \zeta}{\partial \theta^{2}}\right\}
$$

or, in the case of simple-harmonic vibrations,

$$
\frac{\partial^{2} \zeta}{\partial r^{2}}+\frac{1}{r} \frac{\partial \zeta}{\partial r}+\frac{1}{r^{2}} \frac{\partial^{2} \zeta}{\partial \theta^{2}}+k^{2} \zeta=0
$$

with the same meaning of $k^{2}$ as before.

* On account of the frequent occurrence of the Bessel's Functions in mathematical physics, especially in two-dimensional problems, great attention has been devoted to them by mathematicians. The difficulty in investigating their properties is much as if we had to ascertain all the properties of the cosine-function from the series

$$
1-\frac{x^{2}}{2 !}+\frac{x^{4}}{4 !}-\ldots
$$

and were ignorant of its connection with the circle. 
Since $\zeta$ is a periodic function of $\theta$, of period $2 \pi$, it can be expanded (for any particular value of $r$ ) in a series of sines and cosines of multiples of $\theta$, thus

$$
\begin{aligned}
\zeta=R_{0}+R_{1} \cos \theta+S_{1} \sin \theta & +\ldots \\
& +R_{8} \cos s \theta+S_{8} \sin s \theta+\ldots ;
\end{aligned}
$$

by Fourier's theorem; and this formula will apply to the whole membrane if the coefficients be regarded as functions of $r$. Moreover on substitution in (8) it appears that each term must satisfy the equation separately. Thus we have a typical solution

$$
\zeta=R_{8} \cos s \theta \cdot \cos (n t+\epsilon)
$$

provided

$$
\frac{\partial^{2} R_{s}}{\partial r^{2}}+\frac{1}{r} \frac{\partial R_{s}}{\partial r}+\left(k^{2}-\frac{s^{2}}{r^{2}}\right) R_{s}=0 .
$$

The solution of this, which is finite for $r=0$, can be found in the form of an ascending series. In the accepted notation we have $R_{8}=A_{8} J_{8}(k r)$, where the function $J_{8}$ is defined by

$J_{8}(z)=\frac{z^{8}}{2^{8} \cdot s !}\left\{1-\frac{z^{2}}{2(2 s+2)}+\frac{z^{4}}{2.4(2 s+2)(2 s+4)}+\ldots\right\}$.

This is known as the Bessel's Function of the sth order, of the first kind. As in the case of (2) there is a second solution which becomes infinite for $r=0$, but in the case of the complete circular membrane this of course is inadmissible. We have then the normal modes

$$
\zeta=A J_{8}(k r) \cos s \theta \cdot \cos (n t+\epsilon),
$$

where $k$ is determined by

$$
J_{8}(k a)=0 .
$$

Similarly, taking a term $S_{8} \sin s \theta$ from (9) we should have been led to the modes

$$
\zeta=B J_{8}(k r) \sin s \theta \cdot \cos (n t+\epsilon),
$$

with the same determination of $k$. Owing to the equality of periods the normal modes are to some extent indeterminate. Thus, for any admissible value of $k$, we may combine (13) and (15) in arbitrary proportions, and obtain

$$
\zeta=C J_{8}(k r) \cos (s \theta+\alpha) \cdot \cos (n t+\epsilon) .
$$


We have here $s$ nodal diameters, given by

$$
s \theta+\alpha= \pm \frac{1}{2} \pi, \pm \frac{3}{2} \pi, \ldots,
$$

and accordingly arranged at intervals of $\pi / s$. Again for every value of $k$ after the lowest we have one or more nodal circles whose radii are given by the roots of lower order. In the case $s=1$, where there is one nodal diameter, we have

$$
k a / \pi=1 \cdot 2197,2 \cdot 2330,3 \cdot 2383,4 \cdot 2411, \ldots
$$

the numbers tending to the form $m+\frac{1}{4}$. The characters of the corresponding modes may be gathered from the annexed

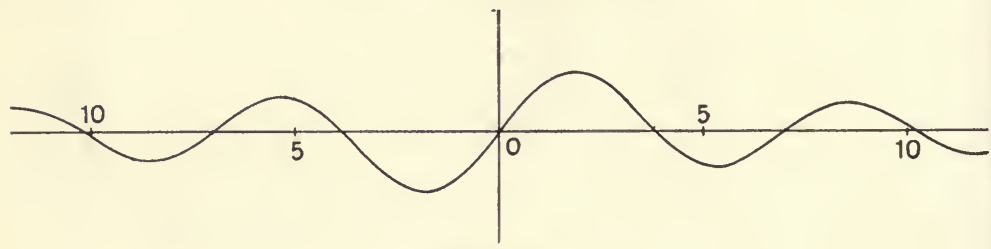

Fig. 52.

graph of the function $J_{1}(z)$; this may be supposed to represent a section through the centre, normal to the nodal diameter. In the second of the above modes, the radius of the nodal circle is given by

$$
r / a=1 \cdot 2197 / 2 \cdot 2330=\cdot 546 .
$$

Fig. 53 shews in plan the configuration of the nodal lines in the first three modes of the types $s=0, s=1, s=2$, respectively. The + and - signs distinguish the segments which are at any instant in opposite phases of vibration.

Whatever the form of the boundary, the value of $\zeta$ in the neighbourhood of any point $O$ of a membrane must admit of expression in the form (9), with

$$
R_{8}=A_{8} J_{s}(k r), \quad S_{8}=B_{8} J_{8}(k r),
$$

the factor $\cos (n t+\epsilon)$ being of course understood. If $O$ be on a nodal line we must have $\zeta=0$ for $r=0$, and therefore $A_{0}=0$. The form of the membrane near $O$ is then given by

$$
\zeta=\left(A_{1} \cos \theta+B_{1} \sin \theta\right) J_{1}(k r),
$$

ultimately, and the direction of the nodal line at $O$ is accord- 
ingly given by $\tan \theta=-A_{1} / B_{1}$. If all the coefficients of order less than $s$ vanish, we have, for small values of $r$,

$$
\zeta=\left(A_{8} \cos s \theta+B_{8} \sin s \theta\right) J_{8}(k r) .
$$

The node has then $s$ branches passing through $O$, making equal angles $\pi / s$ with one another, their directions being given by $\tan s \theta=-A_{\varepsilon} / B_{8}$. This is illustrated in the preceding diagrams; for instance the cases $s=2, s=3, s=4$ all occur in Fig. 50 .
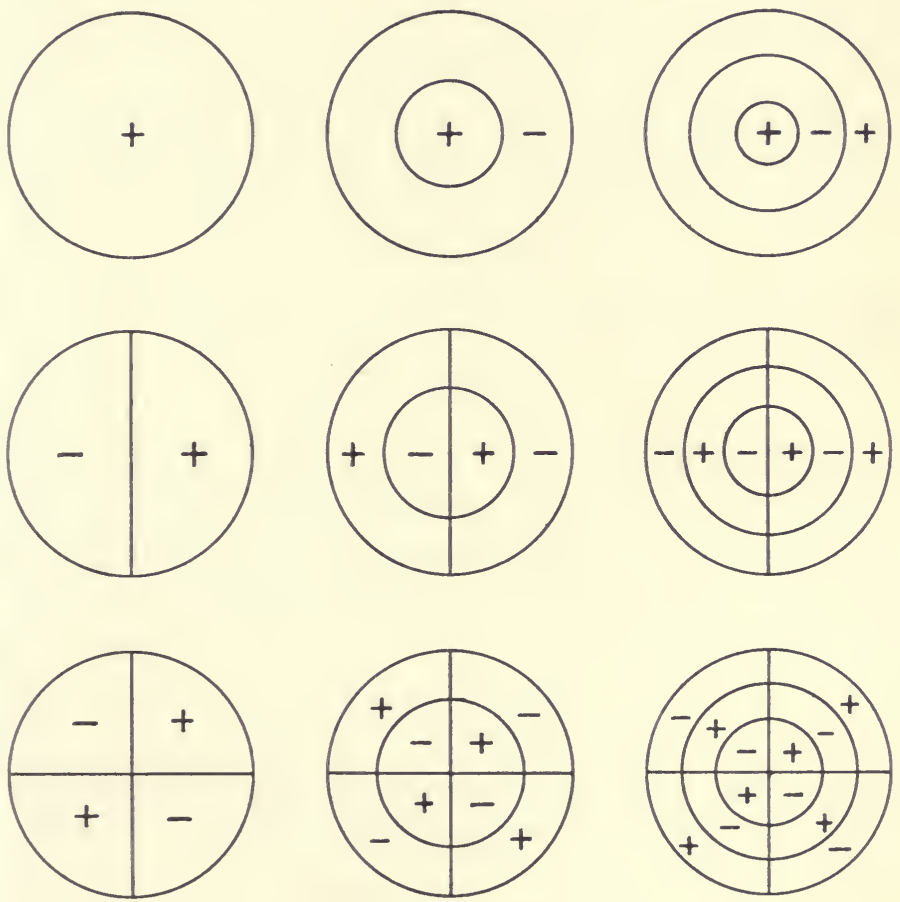

Fig. 53.

According to a general theorem stated in $\$ 16$ it must be possible by combination of the various normal modes of a membrane in suitable proportions, and with proper relations of phase, to represent the effect of arbitrary initial conditions. We do not enter into this; and the theory of the forced vibrations must also be passed over except for a simple example. 
When a force $Z$ per unit area acts on a circular membrane, the equation ( 1 ) is replaced by

$$
\rho \frac{\partial^{2} \zeta}{\partial t^{2}}=P\left(\frac{\partial^{2} \zeta}{\partial r^{2}}+\frac{1}{r} \frac{\partial \zeta}{\partial r}\right)+Z
$$

it being supposed, for simplicity, that there is symmetry as regards the distribution of $Z$ and the consequent displacements $\zeta$. If, further, $Z$ vary as $\cos (p t+\alpha)$, we have

$$
\frac{\partial^{2} \zeta}{\partial r^{2}}+\frac{1}{r} \frac{\partial \zeta}{\partial r}+k^{2} \zeta=-\frac{Z}{P}
$$

where

$$
k^{2}=p^{2} \rho / P \text {. }
$$

If $Z$ be independent of $r$, so that the impressed force is uniform over the membrane, the solution of (22) is obviously

$$
\zeta=-\frac{Z}{k^{2} P}+C J_{0}(k r)
$$

and determining the constant $C$ so that $\zeta=0$ for $r=a$, we find

$$
\zeta=\left\{\frac{J_{0}(k r)}{J_{0}(k a)}-1\right\} \frac{Z}{p^{2} \rho} .
$$

The amplitude becomes very great whenever $k a$ approximates to a root of (5), i.e. whenever the imposed frequency approaches that of one of the symmetrical free modes. When, on the other hand, the imposed vibration is relatively slow, $k a$ is small, and we have by (4)

$$
\zeta=\frac{1}{4} \frac{Z}{P}\left(a^{2}-r^{2}\right)
$$

approximately. This is the statical deflection corresponding to the instantaneous value of the disturbing force.

\section{Uniform Flexure of a Plate.}

The theory of the transverse vibrations of plates stands in the same relation to that of bars as the theory of membranes to that of strings. The reader will understand from this comparison that the mathematical difficulties are considerable, and will not be surprised to learn that some of the most interesting and, at first sight, simple problems remain unsolved. On the other hand the subject readily admits of experimental illustration. If the plate be horizontal, and fixed at one point, the 
configuration of the nodal lines can be exhibited by means of a little sand previously strewn on the surface. When any particular normal mode is excited, the sand is shaken away from the places of vigorous motion, and accumulates in the neighbourhood of the nodal lines. Usually the plate is set into vibration by bowing at right angles to the edge, and the desired mode is favoured by touching the edge with the fingers at one or more nodal points. If, as in the case of a rectangular plate fixed at the centre, the point of support is a nodal point of several normal modes, a great variety of beautiful figures nay be obtained. An extensive series of diagrams of results obtained in this way were given by Chladni; many of these are eproduced in the current manuals of experimental acoustics.

In the theoretical treatment it is assumed that one of the principal axes of strain and stress is normal to the faces of the plate, and that the corresponding stress vanishes. Putting, then, $p_{3}=0$ in the formulae (9) of $\S 42$, we find, for the remaining principal stresses,

$$
p_{1}=E^{\prime}\left(\epsilon_{1}+\sigma \epsilon_{2}\right), \quad p_{2}=E^{\prime}\left(\epsilon_{2}+\sigma \epsilon_{1}\right),
$$

where

$$
E^{\prime}=E /\left(1-\sigma^{2}\right) \text {. }
$$

If $R_{1}, R_{2}$ be the principal radii of curvature at any point of the plate, when bent, we have, by an investigation similar to that of $§ 45$,

$$
\epsilon_{1}=z / R_{1}, \quad \epsilon_{2}=z / R_{2},
$$

where $z$ denotes distance from the medial plane. If we consider a rectangular element of the plate bounded by lines of curvature, and denote by $h$ the half-thickness, this leads to bending moments

$$
\left.\begin{array}{l}
M_{1}=\int_{-h}^{h} p_{1} z d z=\frac{2}{3} E^{\prime} h^{3}\left(\frac{1}{R_{1}}+\frac{\sigma}{R_{2}}\right), \\
M_{2}=\int_{-h}^{h} p_{2} z d z=\frac{2}{3} E^{\prime} h^{3}\left(\frac{1}{R_{2}}+\frac{\sigma}{R_{1}}\right),
\end{array}\right\}
$$

per unit length of the respective edges, in the planes of the two principal curvatures.

The potential energy per unit volume is

$$
\frac{1}{2}\left(p_{1} \epsilon_{1}+p_{2} \epsilon_{2}\right)=\frac{1}{2} E^{\prime}\left(\epsilon_{1}{ }^{2}+2 \sigma \epsilon_{1} \epsilon_{2}+\epsilon_{2}^{2}\right) .
$$


If we substitute from (3), and integrate over the thickness, we find for the potential energy per unit area of the plate

$$
\frac{1}{3} E^{\prime} h^{3}\left(\frac{1}{R_{1}{ }^{2}}+\frac{2 \sigma}{R_{1} R_{2}}+\frac{1}{R_{2}^{2}}\right) .
$$

The formulae (4) may be applied to the case of a flat bar of rectangular section, uniformly bent by two opposing couples $M_{1} b$, where $b$ denotes the breadth. Along the free edges we have $M_{2}=0$, and therefore

$$
R_{2}^{-1}=-\sigma R_{1}^{-1} \text {. }
$$

The bending moment is accordingly

$$
M_{1} b=\frac{2}{3} E b h^{3} / R_{1}
$$

by (4). This agrees, as it must, with $\S 45$ (5), since $\omega=2 b h$, $\kappa^{2}=\frac{1}{3} h^{2}$. The formula (7) shews that when a bar of rectangular section is bent in a plane parallel to one pair of faces, an opposite or "anticlastic" curvature is produced in the plane of the crosssection, the ratio of the curvatures being identical with Poisson's ratio $\sigma$. This circumstance has been made the basis of practical methods of determining $\sigma$, by

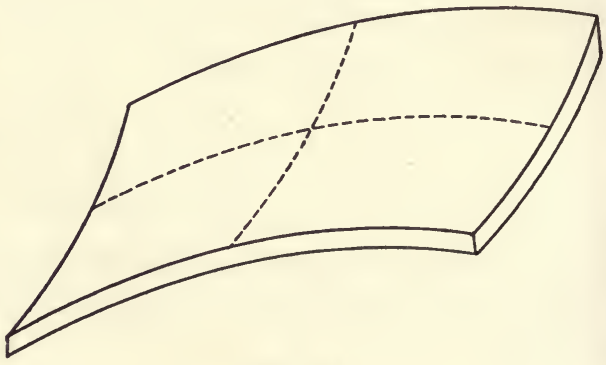

Fig. 54.

Cornu* (1869) and Mallock (1879), the curvatures being measured by optical or other means.

It follows from the above that a perfectly free rectangular plate cannot vibrate after the manner of a bar, with nodal lines parallel to one pair of opposite edges, since couples would be required, about the remaining edges, to counteract the tendency to anticlastic curvature.

\section{Vibrations of a Plate. General Results.}

In a vibrating plate the directions and amounts of the principal curvatures will in general vary from point to point.

* A. M. Cornu (1841-1902), professor of physics at the École Polytechnique 1871-1902. Famous for his experimental determination of the velocity of light, and for other important contributions to optics. 
Shearing forces will also be called into play normal to the plane of the plate. The circumstances are somewhat complicated, but the deduction of the equation of motion for the body of the plate is a straightforward matter, and presents no real difficulty. A more serious question arises when we come to the conditions to be satisfied at a free edge. It appears that the simple condition of strain which has been postulated as the basis of the formulae (4) of $\S 55$ cannot be assumed to hold, even approximately, right up to the edge. In the immediate neighbourhood of the edge, i.e. to a distance inwards comparable with the thickness, a peculiar state of strain in general exists, one remarkable result of which is a shearing force on sections perpendicular to the edge, of quite abnormal amount.

For the further development of the subject reference must be made to other works*. We merely quote a few of the more important results which have been obtained, relating chiefly to plates whose edges are free.

It is found that for a plate of given lateral dimensions the frequency $(n / 2 \pi)$ of any particular normal mode is given by

$$
n^{2}=\frac{1}{3} \frac{E^{\prime}}{\rho} \cdot h^{2} \cdot m^{4},
$$

where, as in $§ 46, m$ is a constant, of the nature of the reciprocal of a line, given by a certain transcendental equation, and $\rho$ denotes the volume-density. For plates with geometrically similar boundaries the frequency accordingly varies as the thickness, and inversely as the square of the lateral dimensions.

In the case of a perfectly free circular disk the nodal lines are circles and equidistant diameters. In the symmetrical modes, which were investigated to some extent by Poisson (1829), we have nodal circles alone. Thus in the gravest mode of this type we have a

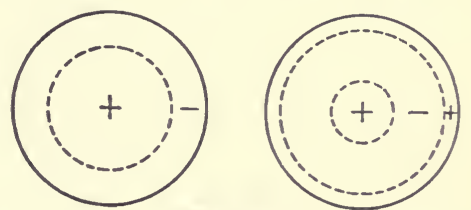

Fig. 55 . nodal circle of radius $678 a$, where $a$ is the radius of the disk; in the next mode there are two nodal circles of radii $392 a$

* See Lord Rayleigh. Theory of Sound, chap. 10 ; Love, Theory of Elasticity, Cambridge, 1906, chap. 22. 
and $842 a$, and so on, the numbers varying slightly however with the value adopted for $\sigma$. According to Poisson, the values of $m$ for the above modes are given by

$$
m^{2} a^{2}=8.8897,38.36,
$$

on the hypothesis that $\sigma=\frac{1}{4}$.

The complete theory of the free circular plate was worked out by Kirchhoff in a celebrated memoir (1850). It appears that the gravest of all the normal modes has two nodal diameters, and no nodal circle. Its frequency is

$$
.523 \frac{h}{a^{2}} \sqrt{ }\left(\frac{E}{\rho}\right) \text { or } 517 \frac{h}{a^{2}} \sqrt{ }\left(\frac{E}{\rho}\right),
$$

according as we adopt the value $\sigma=\frac{1}{4}$ or $\sigma=\frac{1}{3}$ of Poisson's ratio. The figure shews the configuration of the nodal lines in the simplest cases of one and two nodal diameters.

The theory of a circular plate clamped at the edge has been treated by Poisson and

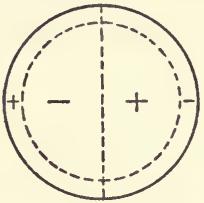

Fig. 56 .

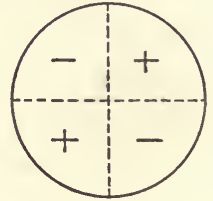
others. In the first two symmetrical modes it is found that

$$
m^{2} a^{2}=10 \cdot 2156,39.59,
$$

respectively. In the second of these modes there is a nodal circle of radius $381 a$. The theory has been applied by Lord Rayleigh to calculate the natural frequencies of a telephone plate.
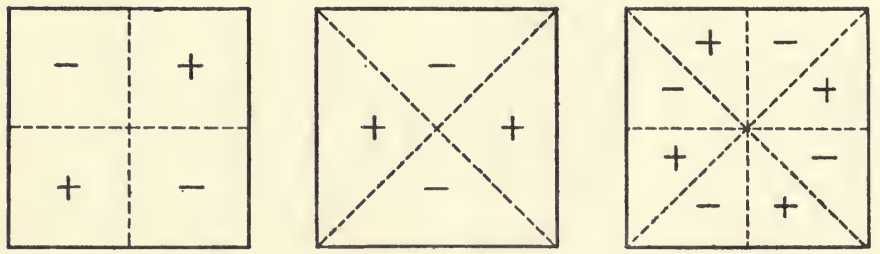

Fig. 57.

In the case of a square plate we have to depend almost wholly on observation, there being at present no exact theory. As in the case of the square membrane ( $\$ 53)$, the nodal lines 
may assume a great variety of forms, owing to the superposition of different modes having the same frequency. The gravest mode of a free plate is that in which the nodal lines form a cross through the centre, with arms parallel to the sides. The figure shews other cases in which possible forms can be assigned to the nodal lines from considerations of symmetry.

\section{Vibrations of Curved Shells.}

When we proceed to the vibrations of curved plates, or shells, we meet with further complications due to the fact that no absolutely sharp line can be drawn between flexural and extensional modes. This has been already exemplified in the case of the ring ( $(51)$. It appears, however, that as the thickness is (in imagination) reduced the normal modes tend to fall into two distinct categories. In one of these the frequencies tend to definite limits, the deformations being mainly extensional, and so analogous to the longitudinal vibrations of a bar, where the dimensions of the cross-section were found to be immaterial. In the second category the frequencies diminish without limit, being ultimately proportional to the thickness, as in the flexural vibrations of a bar or plate.

It will be understood that, acoustically, the flexural vibrations are alone of real interest. When the shape is one of revolution about an axis, the nodal lines will evidently be parallels of latitude and equidistant meridians. As in the case of $§ 51$ these are not lines of absolute rest, the tangential motion being there relatively at its greatest. This has an application to bells. A theoretical calculation of the frequencies of an actual bell is of course out of the question; but it is somewhat remarkable that no systematic experimental study appears to have been made until the subject was taken up by Lord Rayleigh in 1890. Some unexpected results were obtained. To quote a typical case, the normal modes of a particular bell, when arranged in ascending order of frequency, were found to have the following numbers of nodal meridians and parallels, and the pitches indicated:
$(4,0)$
$(4,1)$
$(6, ?)$
$(6, ?)$
$(8, ?)$
$e^{\prime}$
$c^{\prime \prime}$
$f^{\prime \prime}+$
$b^{\prime \prime} b$
$d^{\prime \prime \prime}$. 
Of these the only one which has any relation to the nominal pitch $\left(d^{\prime \prime}\right)$ of the bell is the fifth in order, and this is out by an octave. A mistake of an octave in judging pitch is not uncommon, for physiological reasons, but it is surprising that the presence of the lower dissonant tones should be so easily disregarded. It is conceivable that the mode of striking may be in some degree unfavourable to the production of the more discordant elements.

The vibrations of an elastic solid whose dimensions are all of the same order of magnitude are from our present point of view of subordinate interest. The only case which has been worked out is that of the sphere. In the most important mode one diameter extends and contracts whilst the perpendicular diameters simultaneously contract and expand, respectively. The frequency of this mode is, for such values of $\sigma$ as are commonly met with,

$$
N=840 \frac{\sqrt{ }(\mu / \rho)}{2 a},
$$

about, where $a$ is the radius. This is the lowest of all the natural frequencies. For a steel ball one centimetre in radius, this makes $N=136000$. 


\section{CHAPTER VI}

\section{PLANE WAVES OF SOUND}

\section{Elasticity of Gases.}

In any fluid there is a definite relation between the pressure $p$, the density $\rho$, and the temperature $\theta$, and any two of these quantities accordingly serve to specify the physical state of the substance. It is often convenient to use in place of $\rho$ its reciprocal $v$, the volume of unit mass.

In thermodynamical investigations the two quantities usually chosen as independent variables are $p$ and $v$. In Watt's "indicator diagram" these are taken as rectangular coordinates, $p$ being the ordinate and $y$ the abscissa. Any particular state is then represented by a point on the diagram, and any succession of states by a continuous line. We may imagine the unit mass of the fluid to be enclosed in a deformable envelope, and that an infinitesimal change of volume is produced by a displacement of the boundary in the direction of the normal, whose amount is (say) $\nu$ for any given surfaceelement $\delta S$. The work done by the contained gas in this process is $\Sigma(p \delta S . \nu)$, or $p \delta v$, since $\Sigma(\nu \delta S)=\delta v$. Hence the work done in any succession of changes, represented by a curve on the diagram, will be given by $\int p d v$, i.e. by the area included between the curve, the axis of abscissae, and the first and last ordinates. This area is of course to be taken with its proper sign, according as the work is positive or negative.

There are two kinds of successions of states which are specially important. In the first of these the temperature does not vary, and the representative lines are therefore called "isothermals." By means of a system of isothermal lines drawn at sufficiently small intervals the properties of the 
substance can be completely mapped out. The other successions referred to are those in which there is no gain or loss of heat to the substance, as if it were enclosed in a vessel (o variable volume) whose walls are absolute non-conductors. The corresponding lines are therefore called "adiabatics."

In a perfect gas we have

$$
p=R \rho \theta, \text { or } p v=R \theta,
$$

where $\theta$ is the absolute temperature on the gas thermometer and $R$ is a constant depending on the nature of the gas. The isothermal lines $p v=$ const. are therefore rectangular hyperbolas asymptotic to the coordinate axes. As regards the adiabatics the heat required to increase the pressure by $\delta p$ when the volume is constant will be given by an expression of the form $P \delta p$. If $c$ denote the specific heat (per unit mass) at constant volume, this must be equal to $c \delta \theta$, where $\delta \theta$ is the corresponding change of temperature. Now when $\delta v=0$ we have $\delta p / p=\delta \theta / \theta$, whence, comparing, $P=c \theta / p$. Again, the heat required to augment the volume by $\delta v$ when the pressure is constant. may be denoted by $Q \delta v$, which must be equal to $c^{\prime} \delta \theta$ where $c^{\prime}$ is the specific heat at constant pressure. Since, when $\delta p=0$ we have $\delta v / v=\delta \theta / \theta$, we find $Q=c^{\prime} \theta / v$. The heat absorbed when both pressure and volume are varied infinitesimally is therefore

$$
P \delta p+Q \delta v=\theta\left(c \frac{\delta p}{p}+c^{\prime} \cdot \frac{\delta v}{v}\right)
$$

and the differential equation of the adiabatics is therefore

$$
\frac{\delta p}{p}+\frac{c^{\prime}}{c} \frac{\delta v}{v}=0
$$

The ratio $c^{\prime} / c$ of the two specific heats is practically constant. Denoting it by $\gamma$, we have

$$
\log p+\gamma \log v=\text { const., }
$$

or

$$
p v^{\gamma}=\text { const., }
$$

as the equation of the adiabatic lines. The value of $\gamma$ as found by direct experiment is about $\mathbf{1 . 4 1}$ for air, oxygen, nitrogen, and hydrogen. The figure shews the isothermal and adiabatic lines through a point $P$ of the diagram, the latter curve being the steeper. 
When the pressure and volume vary in any connected manner, the ratio of the increment $\delta p$ of the pressure to the "compression," i.e. the negative dilatation $-\delta v / v$, may be called the " elasticity of volume." Its value will depend not only on the particular state, but on the manner in which the variations from that state are supposed to take place, i.e. on the direction of the corresponding curve on the diagram. If the tangent at the

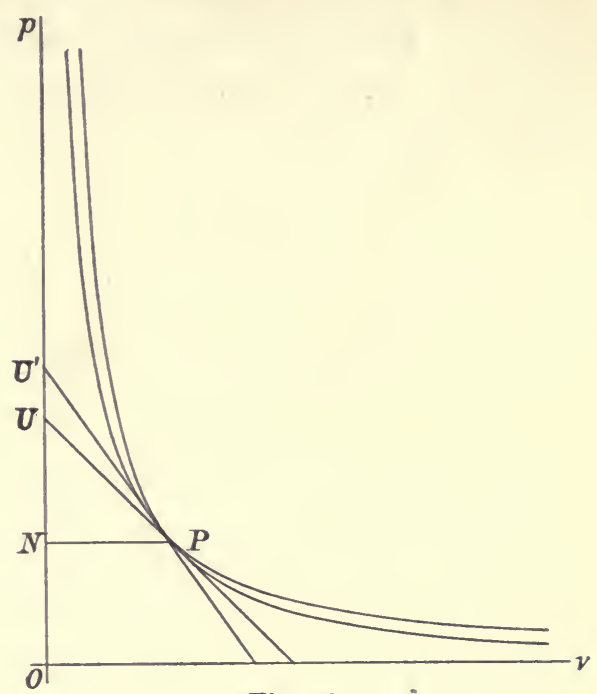

Fig. 53. point $P$ meet the axis of $p$ in $U$, and $N U$ be the projection of $P U$ on this axis, we have

$$
-v \frac{d p}{d v}=N P \cdot \tan U P N=N U
$$

this projection therefore represents the elasticity under the particular condition. On the isothermal hypothesis, to which these letters refer in the figure, the elasticity is equal to the pressure $p$, as follows at once from (1), or from the fact that the tangent to a rectangular hyperbola is bisected at the point of contact. If the variations are subject to the adiabatic law, the elasticity, as deduced from (4), is $\gamma p$, and so greater than in the former case. This is represented by $N U^{\prime}$ in the figure. Even in the case of solid and liquid bodies we ought, in strictness, to discriminate between isothermal and adiabatic coefficients of elasticity, but the differences happen not to be very important.

The work done by unit mass of a gas in expanding between any two adjacent states is easily read off from a diagram as

$$
\frac{1}{2}\left(p+p_{0}\right)\left(v_{0}-v\right), \text { or } p_{0}\left(v_{0}-v\right)+\frac{1}{2}\left(p-p_{0}\right)\left(v_{0}-v\right),
$$


correct to the second order of small quantities. When the two states are a finite distance apart we require to know the manner of transition. For changes along an isothermal line $p v=p_{0} v_{0}$ we have

$$
\int_{v}^{v_{0}} p d v=p_{0} v_{0} \log \frac{v_{0}}{v}
$$

For variations along an adiabatic

$$
\int_{v}^{v_{0}} p d v=\frac{p v-p_{0} v_{0}}{\gamma-1}
$$

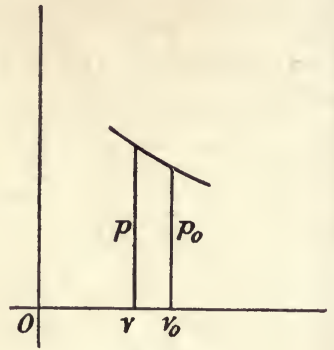

Fig. 59 .

\section{Plane Waves. Velocity of Sound.}

The theory of plane waves of sound is very similar to that of the longitudinal vibrations of rods $(\$ 43)$. We assume that the motion is everywhere parallel to the axis of $x$, and is the same at any given instant over any plane perpendicular to this axis. We denote displacement from the equilibrium position by $\xi$. The symbols $p, \rho, \xi$ are supposed to refer at the time $t$ to that plane of particles whose undisturbed position is $x$; they are therefore functions of the independent variables $x$ and $t$. The constant equilibrium values of $p, \rho$ are distinguished as $p_{0}, \rho_{0}$.

The dilatation $\Delta$ was defined in $\S 40$ as the ratio of the increment of volume to the original volume, viz.

$$
\Delta=\left(v-v_{0}\right) / v_{0}, \quad v=v_{0}(1+\Delta) .
$$

In the present branch of the subject it is usual to introduce a symbol $s$ to denote the "condensation," i.e. the ratio of the increment of density to the original density; thus

$$
s=\left(\rho-\rho_{0}\right) / \rho_{0}, \quad \rho=\rho_{0}(1+s) .
$$

Since $v=1 / \rho$, we have

$$
(1+s)(1+\Delta)=1 \text {. }
$$

The stratum of air which was originally bounded by the planes $x$ and $x+\delta x$ is at time $t$ bounded by the planes $x+\xi$ and 
$x+\xi+\delta x+\delta \xi$, and its thickness is therefore changed from $\delta x$ to $\delta x+\delta \xi$, or $(1+\partial \xi / \partial x) \delta x$, and the dilatation is accordingly

$$
\Delta=\frac{\partial \xi}{\partial x} \text {. }
$$

Hence, in the case of infinitely small disturbances, we have, by (3),

$$
s=-\Delta=-\frac{\partial \xi}{\partial x}
$$

In forming the equation of motion we assume that the pressure varies with the density according to some definite law. We have then, for small values of $s$,

$$
p=p_{0}+\kappa s,
$$

where $\kappa$ is a coefficient of cubic elasticity. Considering the acceleration of momentum of unit area of a stratum originally bounded by the planes $x$ and $x+\delta x$, we have

$$
\rho_{0} \delta x \cdot \ddot{\xi}=-\delta p,
$$

where $\delta p$ represents the excess of pressure on the anterior face. Hence, by (5) and (6),

where

$$
\begin{gathered}
\frac{\partial^{2} \xi}{\partial t^{2}}=c^{2} \frac{\partial^{2} \xi}{\partial x^{2}}, \\
c=\sqrt{ }\left(\kappa / \rho_{0}\right) .
\end{gathered}
$$

The solution of (7) is as in $\$ 23,43$

$$
\xi=f(c t-x)+F(c t+x),
$$

and represents two systems of waves travelling in opposite directions with the velocity $c^{*}$.

If we assume, as Newton + did, that, the expansions and contractions of a gas, as a sound-wave passes, take place isothermally, i.e. without variation of temperature, the relation between $p$ and $\rho$ is given by Boyle's law, viz. $p / p_{0}=\rho / \rho_{0}=1+s$, whence $\kappa=p_{0}$, as already proved. This makes

$$
c=\sqrt{ }\left(p_{0} / \rho_{0}\right) \text {. }
$$

Now for air at $0^{\circ} \mathrm{C}$. we may put, as corresponding values,

$$
p_{0}=76 \times 13.60 \times 981, \quad \rho_{0}=\cdot 00129,
$$

* The analytical theory of plane waves of sound is due to Euler (1747) and Lagrange (1759).

+ The investigation is given in Prop. 48 of the second book of the Principia (1726). 
in absolute centimetre-gramme-second units, whence $c=\mathbf{2 8 0}$ metres per second. This is considerably less than the observed velocity.

The discrepancy was first fully accounted for by Laplace and Poisson*. When a gas is rarefied or condensed the temperature tends to fall or rise, except in so far as the process is mitigated by the supply or abstraction of heat. In ordinary sound-waves the condensation $s$ changes sign so frequently, and the temperature consequently rises and falls so rapidly, that there is no time for sensible transfer of heat between adjacent portions of the gas. The flow of heat has hardly set in from one element to another before its direction is reversed, and the conditions are therefore practically adiabatic. The formula

$$
p / p_{0}=\left(\rho / \rho_{0}\right)^{\gamma}
$$

becomes, for small values of $s$,

$$
p=p_{0}(1+\gamma s)
$$

whence $\kappa=\gamma p_{0}$, as in $\S 58$, and

$$
c=\sqrt{ }\left(\gamma p_{0} / \rho_{0}\right) .
$$

Putting $\gamma=1.41$ we find that the Newtonian velocity of sound in air must be increased in the ratio $1 \cdot 187$, whence $c=332$ metres per second at $0^{\circ} \mathrm{C}$. This is in good agreement with direct observation.

As there is now no question as to the soundness of this explanation, and as the direct determination of $\gamma$ is a matter of considerable difficulty, the formula (13) is often used in the inverse manner, as a means of deducing the value of $\gamma$ for various gases from the observed velocities of sound-waves in them. For example, it was in this way that in 1895 the value of $\gamma$ for the newly discovered gas argon was found by Lord Rayleigh to lie between $1 \cdot 6$ and $1 \cdot 7$. The experimental method (due to Kundt) is referred to in $\S 62$ below.

Since $p_{0} / \rho_{0}=R \theta_{0}$, the velocity of sound as given by (13) is independent of the actual density, but will vary as the square root of the absolute temperature. Also, so far as $\gamma$ has the same value, the velocity of sound in different gases will vary

* About, or before, the year 1807. 
inversely as the square root of the density, provided the comparison be made at the same pressure. These conclusions are in agreement with observation.

The formula (8) will of course apply to any fluid medium, provided the proper value of $\kappa$ be taken. In liquids the difference between the isothermal and adiabatic elasticities may be neglected. For water at $15^{\circ} \mathrm{C}$. we may put $\kappa=2.22 \times 10^{10}, \rho_{0}=1$, in C.G.S. units, whence $c=1490$ metres per second. The number found by Colladon and Sturm (1826) by direct observation, in the water of the lake of Geneva, was 1435, at a temperature of about $8^{\circ} \mathrm{C}$.

Another formula for the velocity of sound may be noticed. If $H$ denote the height of a "homogeneous atmosphere," i.e. of a column of uniform density $\rho_{0}$ whose weight would produce the actual pressure $p_{0}$ per unit area, we have $p_{0}=g \rho_{0} H$, and the Newtonian formula (10) becomes

$$
c=\sqrt{ }(g H)
$$

cf. $\S 43(6)$. The velocity is accordingly that due to a fall from rest through a height $\frac{1}{2} H$. It appears from $\S 58(1)$ that for a given gas, and at a given place, $H$ depends only on the temperature. The corresponding adiabatic formula is

$$
c=\sqrt{ }(\gamma g H) \text {. }
$$

\section{Energy of Sound-Waves.}

The kinetic energy of a system of plane waves is, per unit area of the wave-fronts,

$$
T=\frac{1}{2} \rho_{0} \int \dot{\xi}^{2} d x,
$$

where the integration extends over the space which was occupied by the disturbed air in the equilibrium state.

The work done by unit mass in expanding through a small range was found in $\$ 5 \$$ to be given accurately, to the second order, by the expression

$$
p_{0}\left(v_{0}-v\right)+\frac{1}{2}\left(p-p_{0}\right)\left(v_{0}-v\right),
$$

where the suffix 0 refers to the final state. If we form the sum of the corresponding quantities for all the mass-elements of the system, the first term disappears whenever the conditions are such that the total change of volume is zero. Again, in the 
second term we may put, with sufficient accuracy, $p-p_{0}=\kappa s$, $v_{0}-v=v_{0} s$, and obtain $\frac{1}{2} \kappa s^{2} \cdot v_{0}$. The expression $\frac{1}{2} \kappa s^{2}$ is therefore to be integrated over the volume occupied in the undisturbed state. So far nothing is stipulated as to the hypothesis to which $\kappa$ relates; but it is only in the case of adiabatic expansion that the result can be identified with the potential energy in the strict sense of this term. We then have

$$
V=\frac{1}{2} \kappa \int s^{2} d x
$$

where $\kappa=\gamma p_{0}$, per unit area of wave-front. If $\kappa$ refer to the isothermal condition, the expression on the right hand is what is known in thermodynamics as the "free energy."

It is unnecessary to repeat what has been said in $\$ 23$ as to the resolution of an arbitrary initial disturbance into two wave-systems travelling in opposite directions. In a single progressive wave-system, say

$$
\xi=f(c t-x),
$$

we have by $\S 59(5)$

$$
\dot{\xi}=c s,
$$

where $\dot{\xi}$ denotes the particle-velocity in the direction of propagation. Since $\dot{\xi}$ has the same sign as $s$, an air-particle moves forwards (i.e. with the waves) as a phase of condensation passes it, and backwards during a rarefaction. It appears moreover, from (1), (3), and (5), that the total energy is half kinetic and half potential. This also follows independently from the general argument given in $§ 23$.

The case of a simple-harmonic train of progressive waves is specially important. The formula

$$
\xi=a \cos n\left(t-\frac{x}{c}\right)
$$

represents a train of amplitude $a$, frequency $n / 2 \pi$, and wavelength $\lambda=2 \pi c / n$. We find

$$
\begin{aligned}
T & =\frac{1}{2} \rho_{0} n^{2} a^{2} \int \sin ^{2} n\left(t-\frac{x}{c}\right) d x \\
& =\frac{1}{4} \rho_{0} n^{2} a^{2} \int\left\{1-\cos 2 n\left(t-\frac{x}{c}\right)\right\} d x .
\end{aligned}
$$

The mean value of the second term under the integral sign is zero, and the average kinetic' energy per unit volume is therefore $\frac{1}{4} \rho_{0} n^{2} a^{2}$, and the average value of the total energy $\frac{1}{2} \rho_{0} n^{2} a^{2}$. 
Since $n a$ is the maximum particle-velocity, we see that the energy in any region including an exact number of wave-lengths is the same as the kinetic energy of the whole mass when animated with the maximum velocity of the air-particles. If $s_{\mathbf{1}}$ be used to denote the maximum condensation, we have $s_{1}=n a / c$, and the average energy per unit volume may therefore also be expressed by $\frac{1}{2} \rho_{0} c^{2} s_{1}^{2}$.

We can also estimate, incidentally, the nature of the approximation involved in the derivation of the equation of motion $\$ 59$ (7). The approximation consisted in neglecting the square of $s$, or $-\partial \xi / \partial x$. Since $s_{1}=2 \pi a / \lambda$, this means that the amplitude $a$ is assumed to be small compared with $\lambda / 2 \pi$, a condition which is abundantly fulfilled in all ordinary sound-waves.

So far we have traced the course of waves regarded as already existent, without any reference to their origin. As an example, though a somewhat artificial one, of the manner in which waves may be supposed to be generated, imagine a long straight tube, of sectional area $\omega$, in which a piston is made to move to and fro through a small range, in any arbitrary manner. The origin of $x$ being taken at the mean position of the piston, the forced waves in the tube, to the right, due to a prescribed motion

$$
\xi_{0}=f(t)
$$

of the piston, will evidently be given by

$$
\xi=f\left(t-\frac{x}{c}\right) \text {. }
$$

In particular, if

$$
\xi_{0}=a \cos n t,
$$

we have

$$
\xi=a \cos n\left(t-\frac{x}{c}\right) \text {. }
$$

The rate at which work is being done by the piston on the air to the right is

$$
\begin{aligned}
p \omega \dot{\xi}_{0} & =\left(p_{0}+\kappa s\right) \omega \dot{\xi}_{0} \\
& =-p_{0} \omega n a \sin n t+\frac{\kappa n^{2} a^{2}}{c} \omega \sin ^{2} n t .
\end{aligned}
$$

The mean value of the first term is zero, whilst that of the second is

$$
\frac{1}{2} \kappa n^{2} a^{2} \omega / c=\frac{1}{2} \rho_{0} n^{2} a^{2} \omega c
$$


This is exactly the mean energy contained in a volume $\omega c$ of the space occupied by the wave-train (11). The result may perhaps at first sight appear to be a mere truism. It may be argued that in each unit of time fresh waves are generated which occupy a length $c$ of the tube, and that the piston must as a matter of course supply the corresponding amount of energy. It must be remembered, however, that an infinitely long train of waves of the type (11) would take an infinite time to establish, and that in the case of a finite train the suggested line of argument would require us to examine into what is taking place at its front. In the present instance the result would, it is true, be unaffected, but the case would be altered if the wave-velocity were different for different wave-lengths, as it is for example in dispersive media in optics, in deep-water waves in hydrodynamics, and in the case of flexural waves on a long straight bar (\$45). There is then a distinction between the wave-velocity (for a particular wave-length) and the "groupvelocity" which determines the rate of propagation of energy.

In the above problem force must be applied to the piston in order to maintain the vibration (8) against the reaction of the air. If the piston be free, the store of energy which it originally possessed will be gradually used up in the generation of air-waves. Suppose, for example, that the piston is attached to a spring, and that in the absence of the air the period of its free vibrations would be $2 \pi / n$. Under the actual conditions, its equation of motion will be of the form

$$
M\left(\ddot{\xi}+n^{2} \xi\right)=-\left(p-p_{0}\right) \omega,
$$

where the variable part of the pressure alone appears, since the constant part merely affects the equilibrium position. From the general theory of progressive waves we have

$$
p-p_{0}=\kappa s=\kappa \dot{\xi} / c,
$$

and the equation (14) becomes

$$
\ddot{\xi}+\frac{\kappa \omega}{M c} \dot{\xi}+n^{2} \xi=0 .
$$

This is of the form discussed in $\S 11$, and the solution is

$$
\xi=C e^{-t / \tau} \cos \left(n^{\prime} t+\epsilon\right),
$$


provided $\tau=2 M c / \kappa \omega=2 M / \rho_{0} \omega c, n^{\prime 2}=n^{2}-1 / \tau^{2}$. …...(18)

When $n \tau$ is large the effect on the period may be neglected. The condition for this is that $2 M^{*}$ must be large compared with $\rho_{0} \omega \lambda / 2 \pi$, where $\lambda$ is the wave-length. The inertia of the piston must therefore be great compared with that of the air contained in a length $\lambda / 2 \pi$ of the tube. The same law of decay would be given also by the indirect method explained in $\S 12$.

We have seen in (13) that the rate of propagation of energy across unit area of wave-front in a progressive system of waves of simple-harmonic type is $\frac{1}{2} \rho_{0} n^{2} a^{2} c$, or $\frac{1}{2} \rho_{0} c^{3} s_{1}{ }^{2}$, if $s_{1}$ denote the maximum condensation. The result was obtained for plane waves, but will hold for all kinds of wave at a sufficient distance from the source. Consequently if $W$ denote the total emission of sonorous energy per second from a source near the ground, the value of $s_{1}$, at a distance $r$, will be given by the relation

$$
W=\frac{1}{2} \rho_{0} c^{3} s_{1}^{2} \times 2 \pi r^{2}=\pi \rho_{0} c^{3} r^{2} s_{1}^{2} .
$$

This formula was applied by Lord Rayleigh to estimate the limit of audibility of a sound of given pitch. The value of $W$, as inferred from the power spent in actuating the source (a whistle), is the product of the current into the pressure, and if $r$ be the distance at which the sound is just audible, the formula will give a value of $s_{1}$, which is necessarily, however, greater than the true limit, since the value of $W$ is too high, not all the energy being spent in sound. In this way it was ascertained that sounds could be heard in which $s_{1}$ was certainly less than $4 \times 10^{-8}$. The corresponding amplitude as deduced from the formula $n a=c s_{1}$, was $8 \times 10^{-8} \mathrm{~cm}$. By an independent method, in which the above source of uncertainty was avoided, the limit of audibility was fixed at about $s_{1}=6 \times 10^{-9}$. Subsequent experiments by Wien (1903) and Rayleigh $†$ indicate an increase of sensitiveness with rise of pitch, for tones near the middle of the ordinary musical scale.

* The factor 2 would disappear if the piston were supposed to generate waves on both sides.

† Phil. Mag. (6), vol. xIv. (1907). 


\section{Reflection.}

When there is a fixed barrier at the origin the general solution is replaced, as in $\S 24$, by

$$
\xi=f(c t-x)-f(c t+x) \text {. }
$$

Considering, for example, the region to the left of the origin, the first term may be interpreted as representing a primary wave-system approaching the barrier; the second term then represents the reflected system. The latter has the same amplitude at corresponding points; the velocity $\dot{\xi}$ is reversed, but the condensation $s(=-\partial \xi / \partial x)$ has its sign unchanged. We have here, in its simplest form, the explanation of echoes.

There is another case of reflection which it is important to consider. Suppose that at one point (say $x=0$ ) the condition of unvarying pressure $(s=0)$ is imposed. We must have then, in $§ 59(9)$,

$$
F^{\prime}(c t)=f^{\prime}(c t),
$$

which shews that the functions $f, F$ must differ only by a constant. Since this constant would merely represent a displacement common to the whole mass, which is without influence on the question, it may be ignored. We have then

$$
\xi=f(c t-x)+f(c t+x),
$$

where as before the first term may be taken to represent an incident, and the second a reflected wave-system, in the region lying to the left of 0 . The velocity $\dot{\xi}$ is here reflected unchanged, but the sign of $s$ is reversed. The conditions would be realized if the air were in contact at the plane $x=0$ with a medium capable of exerting pressure, but destitute of inertia. This is of course an ideal case, but the condition of invariable pressure is approximated to in some degree at the open end of a pipe. The present investigation has also an application to the reflection of longitudinal waves at the free end of a rod (§ 43).

The general problem of (direct) reflection at the common boundary of two distinct fluid media is hardly more complicated. The origin being taken in the boundary, a wave-system approaching from the left will give rise to a reflected wave on the left and a transmitted wave on the right. We distinguish 
quantities relating to the incident and reflected wave by the suffixes 1 and 2, respectively, whilst those relating to the transmitted wave are indicated by (grave) accents. Since the velocity and the pressure must be the same for the two media at the origin, we have

$$
\dot{\xi}_{1}+\dot{\xi}_{2}=\dot{\xi}, \quad \kappa s_{1}+\kappa s_{2}=\kappa^{\prime} s^{\prime} \quad[x=0],
$$

the equilibrium pressure $p_{0}$ being necessarily the same. Now $\dot{\xi}_{1}=c s_{1}, \dot{\xi}_{2}=-c s_{2}, \xi^{\prime}=c^{\prime} s^{\prime}$, whence

$$
c\left(s_{1}-s_{2}\right)=c^{\prime} s^{\prime}, \quad \kappa\left(s_{1}+s_{2}\right)=\kappa^{\prime} s^{\prime} \quad[x=0] .
$$

Hence

$$
s_{2}=\frac{\kappa^{\prime} c-\kappa c^{\prime}}{\kappa^{\prime} c+\kappa c^{\prime}} s_{1}, \quad s^{\prime}=\frac{2 \kappa c}{\kappa^{\prime} c+\kappa c^{\prime}} s_{1} \quad[x=0] .
$$

These formulae relate in the first instance to the state of things at the origin, on the two sides; but it is easily seen that they will also reprent the ratios of amplitudes at corresponding points in the respective waves. If the inertia of the second medium were infinite, we should have $c^{\prime}=0$, and therefore $s_{2}=s_{1}$, as in the case of reflection at a rigid barrier. On the other hand, if the inertia of the second medium were evanescent, we should have $c^{\prime}=\infty$ and $s_{2}=-s_{1}$, as above.

The energies of corresponding portions of the various waves are proportional to $\kappa s_{1}^{2} c, \kappa s_{2}{ }^{2} c, \kappa^{\prime} s^{\prime 2} c^{\prime}$, since the lengths occupied by these portions will vary as the respective wave-velocities. The conservation of energy therefore requires

$$
\kappa s_{1}^{2} c=\kappa s_{2}^{2} c+\kappa^{\prime} s^{\prime 2} c^{\prime}
$$

this is easily verified from (6).

If we put $\kappa=\rho_{0} c^{2}, \kappa^{\prime}=\rho_{0}{ }^{\prime}{ }^{\prime 2}$, we have, from (6),

$$
\frac{s_{2}}{s_{1}}=\frac{\rho_{0}{ }^{\prime} c^{\prime}-\rho_{0} c}{\rho_{0}{ }^{\prime} c^{\prime}+\rho_{0} c} \text {. }
$$

As an example, take the case of air-waves incident normally on the surface of water. We have $\rho_{0} / \rho_{0}{ }^{\prime}={ }^{\prime} 00129, c / c^{\prime}=\cdot 222$, about; whence $s_{2} / s_{1}=.99943$. There is therefore almost complete reflection, with hardly any transmission.

In the case of two gaseous media having the same ratio of specific heats, and therefore the same elasticity $\left(\kappa=\gamma p_{0}\right)$, the formulae simplify; thus

$$
\frac{s_{2}}{s_{1}}=\frac{c-c^{\prime}}{c+c^{\prime}}, \frac{s^{\prime}}{s_{1}}=\frac{2 c}{c+c^{\prime}} .
$$


These are identical with Fresnel's formulae for the amplitudes of reflected and transmitted light in the case of normal incidence on the common boundary of two transparent media.

\section{Vibrations of a Column of Air.}

When we come to the free oscillations of the air contained in a pipe of finite length, the question definitely arises as to the condition to be satisfied at an open end. There is here a transition, more or less rapid, from plane waves in the tube to diverging spherical waves in the external space, which it is difficult to allow for exactly. In the usual rudimentary theory, which dates from D. Bernoulli, Euler, and Lagrange, it is assumed that the variation of pressure in the tube, at the open end, may be neglected. As already stated, this would be accurately the case if the external air were replaced by a substance capable of exerting pressure $\left(p_{0}\right)$ but devoid of inertia. There would then be no loss of energy on reflection at the open end ( $\S 61)$, and the vibrations in the tube, once excited, would be persistent. The hypothesis is obviously an imperfect representation of the facts; the condition $s=0$ can only be approximately fulfilled, and energy must continually be spent in the generation of waves diverging outwards from the mouth, so that the vibrations if left to themselves will be sensible only for a very limited time; this may however cover hundreds of periods. We shall return to these questions later (Chapter IX); at present we content ourselves with tracing out the consequences of the approximate theory.

The periodic character of the motion in a finite pipe can be inferred from the theory of waves, exactly as in the case of strings (§ 24). Suppose for example that a wave of limited extent is started in either direction from a point $P$ of a tube $A B$. After two reflections, at $A$ and $B$, the wave will pass $P$ again in the same direction as at first. If both ends be closed, the sign of $s$ is unaltered at either reflection, whilst that of $\dot{\xi}$ is twice reversed. Hence after the interval $2 l / c$, where $l=A B$, the initial circumstances are exactly reproduced. The same result holds if both ends be open, since there have now been 
two reversals of $s$ and none of $\dot{\xi}$ in the interval in question. But if one end be closed and the other open, the signs of $s$ and $\dot{\xi}$ at $P$ have each undergone one reversal only in the interval $2 l / c$, and a further interval of like duration must elapse before the original state of things at $P$ is restored.

The foregoing theory explains one or two important points in the theory of organ-pipes. Thus the frequency, in the gravest mode, is inversely proportional to the length, and is lower by an octave for a "stopped" pipe, i.e. a pipe closed at one end, than for an "open" pipe, i.e. one open at both ends, of the same length. It is, again, directly proportional to the velocity of sound, and so increases with rise of temperature.

In the analytical method for determining the normal modes we assume as usual that $\xi$ varies as $\cos (n t+\epsilon)$. The equation $\S 59$ (7) then becomes

$$
\frac{\partial^{2} \xi}{\partial x^{2}}+\frac{n^{2}}{c^{2}} \xi=0
$$

the solution of which is

$$
\xi=\left(A \cos \frac{n x}{c}+B \sin \frac{n x}{c}\right) \cos (n t+\epsilon),
$$

as in $§ 25$. The corresponding wave-length of progressive waves in free air is $\lambda=2 \pi c / n$. Hence in any system of standing waves there is a series of nodes $(\xi=0)$ at intervals of $\frac{1}{2} \lambda$, and a series of loops, or places of zero condensation, $(\partial \xi / \partial x=0)$, half-way between these.

For a tube closed at both ends $(x=0, x=l)$ we have

and therefore

$$
A=0, \quad \sin (n l / c)=0,
$$

$$
\xi=C_{m} \sin \frac{m \pi x}{l} \cos \left(\frac{m \pi c t}{l}+\epsilon_{m}\right),
$$

where $m=1,2,3, \ldots$, the normal modes forming a harmonic series.

For a pipe open at both ends, the condition that $s=-\partial \xi / \partial x=0$ for $x=0$ and $x=l$ gives

$$
B=0, \quad \sin (n l / c)=0,
$$

and the typical solution is

$$
\xi=C_{m} \cos \frac{m \pi x}{l} \cos \left(\frac{m \pi c t}{l}+\epsilon_{m}\right),
$$


where $m=1,2,3, \ldots$ Here, again, the sequence of normal modes is harmonic. The figure illustrates the cases $m=1$, $m=2$. The arrows shew the direction of motion at the loops, whose position is indicated by the dotted lines, in two opposite phases; the nodes are indicated by the full transverse lines.

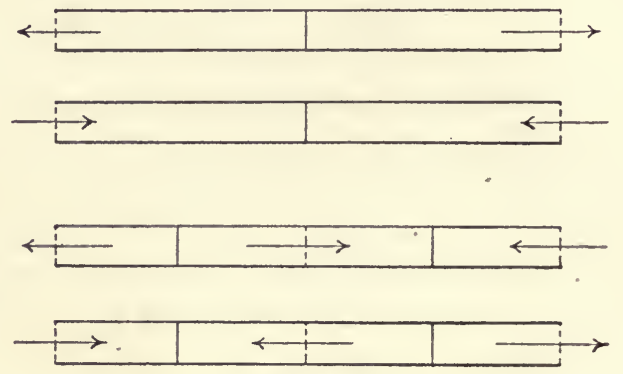

Fig. 60 .

In the case of a pipe closed at $x=0$ and open at $x=l$, we have

$$
A=0, \quad \cos (n l / c)=0,
$$

whence $n l / c=\frac{1}{2} m \pi$, the integers $m$ being odd. We thus obtain

$$
\xi=C_{m} \cos \frac{m \pi x}{2 l} \cos \left(\frac{m \pi c t}{2 l}+\epsilon_{m}\right),
$$

where $m=1,3,5, \ldots$ The absence of the harmonics of even

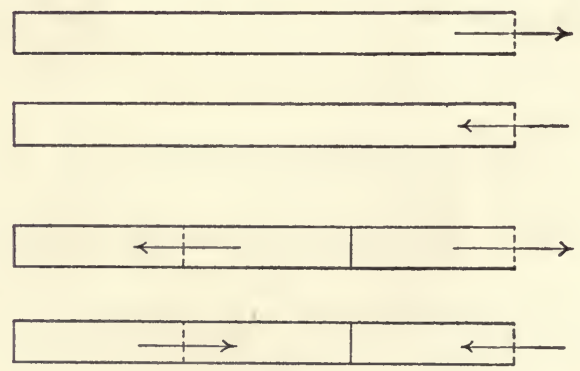

Fig. 61 .

order determines the characteristic "quality" of stopped pipes (§91). The figure shews the cases $m=1, m=3$.

The formula (2) can be applied also to the case of forced 
vibrations of given frequency $(n / 2 \pi)$. Thus if a prescribed vibration

$$
\xi=A \cos (n t+\epsilon)
$$

be maintained at $x=0$, and if the tube be closed at $x=l$, the motion of the gas is given by

$$
\xi=\frac{A}{\sin (n l / c)} \sin \frac{n(l-x)}{c} \cos (n t+\epsilon) .
$$

The amplitude becomes abnormally great, even when we take account of dissipative forces, if $\sin (n l / c)=0$, or $l=\frac{1}{2} m \lambda$, where $m$ is integral. This is the principle of a method due to Kundt (1868) by which the velocity of sound in various gases can be compared by small-scale experiments. The wave-lengths are found by measuring the distances between the nodes, whose position is indicated by the heaping up of lycopodium powder previously scattered in the tube. The vibrations are excited in the two tubes (containing the two gases to be compared) by disks fitted to the two ends of a longitudinally vibrating rod.

If the end $x=l$ is open, the formula (10) is replaced by

$$
\xi=\frac{A}{\cos (n l / c)} \cos \frac{n(l-x)}{c} \cos (n t+\epsilon),
$$

and the condition of strongest resonance is $\cos (n l / c)=0$, or $l=\frac{1}{4} m \lambda$, where $m$ is an odd integer.

The preceding investigations would apply also to the vibrations of a column of water, or other liquid, contained in a tube, provided the material of the tube were absolutely rigid. In practice, however, the yielding of the walls has an appreciable effect; the potential energy corresponding to a given strain $(\partial \xi / \partial x)$ of the fluid is diminished, and the wavevelocity is lowered. The fact was observed by Wertheim (1847), but the true explanation is due to Helmholtz (1848). The question has been further investigated by Korteweg (1878) and the present writer. Owing to the much greater velocities $(\$ 44)$ of elastic waves in solids such as glass or steel, as compared with the sound-velocity in water, the stresses in the walls adjust themselves so rapidly that it is legitimate to assume that the deformation of the tube has the statical value corresponding to the instantaneous distribution of 
pressure in the liquid. If $c_{0}$ be the theoretical velocity of sound in the liquid, as given by $\S 59$ (8), c the actual velocity, it is found that in the case of a tube of small thickness $h$

$$
c^{2}=\frac{c_{0}{ }^{2}}{1+2 \kappa a / h E}
$$

where $a$ is the internal radius, $\kappa$ is the volume-elasticity of the liquid, and $E$ is the value of Young's modulus for the material of the tube. Thus in the case of water $\left(\kappa=2.22 \times 10^{10}\right)$ contained in a glass tube $\left(E=6.03 \times 10^{11}\right)$ whose thickness is one-tenth of the radius, we find $c=\cdot 759 c_{0}$. Even in the other extreme, when the walls are very thick, it. is found that

$$
c^{2} / c_{0}^{2}=\mu /(\kappa+\mu),
$$

where $\mu$ is the rigidity. The value of $\mu$ for glass is, roughly, about 10 times the value of $\kappa$ for water; this would give a diminution of about 5 per cent. in the wave-velocity.

174 63. Waves of Finite Amplitude.

The laws of sound propagation, as they are investigated in this and succeeding chapters, are subject to some qualifications which may best be considered in relation to plane waves, where the theory is simplest.

In the first place, it has been assumed that the condensation $s$ may be treated as infinitely small. This hypothesis is adequate for most purposes, but there are certain "second order" effects which are of some theoretical importance.

It is easy to shew that a progressive wave of finite (as distinguished from infinitely small) amplitude cannot be propagated without change of type, except on the hypothesis of a certain special relation between pressure and density. Assuming, for a moment, that a wave of permanent type is in progress, we may in imagination impress on the whole mass of air a velocity equal and opposite to that of the wave. In this way we obtain a condition of "steady motion" as it is called, in which the velocity, pressure, and density at any point of space are constant with respect to the time. For definiteness we may fix our attention on the air contained in a long straight tube of unit sectional area. The velocity $u$ 
being now a function of the space-coordinate $x$ only, the acceleration of the air-particles will be given by $u d u / d x$ as in ordinary dynamics. Hence, considering the acceleration of momentum of the mass which at the instant considered lies between the planes $x$ and $x+\delta x$, we have

$$
\rho u \frac{d u}{d x}=-\frac{d p}{d x} \text {. }
$$

Also, since the same amount of matter crosses each section in unit time, we have

$$
\rho u=\text { const. }=m,
$$

say. Hence $m d u / d x=-d p / d x$, and

$$
p=C-m u \text {, }
$$

or

$$
p-p_{0}=m\left(u_{0}-u\right)=m^{2}\left(\frac{1}{\rho_{0}}-\frac{1}{\rho}\right),
$$

where the symbols $p_{0}, \rho_{0}, u_{0}$ refer to the parts of the medium which in the original form of the question were undisturbed. This gives the special relation referred to. In terms of the volume per unit mass we have

$$
p-p_{0}=m^{2}\left(v_{0}-v\right)
$$

which is the equation of a straight line on the indicator diagram. A relation of this type does not hold for any known substance, whether under the adiabatic or the isothermal condition, and could in any case only apply to a limited range, since the volume would otherwise shrink to nothing under a certain finite pressure.

If, however, the range of density be small, the equation (5) can be identified with $\S 59(6)$ provided $m^{2}=\kappa \rho_{0}$. Since $m=\rho_{0} u_{0}$, where $u_{0}$ is the wave-velocity in the original form of the problem, this gives $u_{0}^{2}=\kappa / \rho_{0}$, in agreement with $§ 59(8)$. The process is equivalent to choosing $m$ so that the straight line (5) shall be a tangent at the point $\left(v_{0}, p_{0}\right)$ to the curve which on the indicator diagram gives the effective relation between $p$ and $v$.

The condition (5) was obtained in different ways by Earnshaw (1860) and Rankine* (1870).

To ascertain the character of the continual change of type

* W. J. M. Rankine (1820-72), professor of engineering at Glasgow, 1855-72. 
which must take place in sound-waves propagated in actual fluids, we must have recourse to accurate equations of motion. On the plan of $\S 59$ we have

$$
\rho_{0} \frac{\partial^{2} \xi}{\partial t^{2}}=-\frac{\partial p}{\partial x}
$$

and

$$
\rho=\rho_{0} /(1+\Delta)=\rho_{0} /\left(1+\frac{\partial \xi}{\partial x}\right) \text {. }
$$

Hence, on the adiabatic hypothesis ihat

$$
p / p_{0}=\left(\rho / \rho_{0}\right)^{\gamma},
$$

we find by elimination of $p$ and $\rho$

$$
\frac{\partial^{2} \xi}{\partial t^{2}}=c^{2} \frac{\partial^{2} \xi}{\partial x^{2}} /\left(1+\frac{\partial \xi}{\partial x}\right)^{\gamma+1},
$$

where $c^{2}=\gamma p_{0} / \rho_{0}$ as before.

For illustrative purposes it is sufficient to consider the isothermal case, which is derived from the above by putting $\gamma=1$, so that

$$
\frac{\partial^{2} \xi}{\partial t^{2}}=c^{2} \frac{\partial^{2} \xi}{\partial x^{2}} /\left(1+\frac{\partial \xi}{\partial x}\right)^{2}
$$

We have seen in $\S 60$ that on the hypothesis of infinitely small vibrations there is a definite relation between particlevelocity and condensation in a progressive wave. Following Earnshaw, we assume (tentatively) that the same thing holds in the general case, and write accordingly

$$
\frac{\partial \xi}{\partial t}=f\left(\frac{\partial \xi}{\partial x}\right)
$$

where the form of the function is to be determined. From this we deduce

$$
\frac{\partial^{2} \xi}{\partial t^{2}}=f^{\prime}\left(\frac{\partial \xi}{\partial x}\right) \frac{\partial^{2} \xi}{\partial x \partial t}, \quad \frac{\partial^{2} \xi}{\partial x \partial t}=f^{\prime}\left(\frac{\partial \xi}{\partial x}\right) \frac{\partial^{2} \xi}{\partial x^{2}},
$$

and therefore $\quad \frac{\partial^{2} \xi}{\partial t^{2}}=\left\{f^{\prime}\left(\frac{\partial \xi}{\partial x}\right)\right\}^{2} \cdot \frac{\partial^{2} \xi}{\partial x^{2}}$.

Hence (10) is satisfied provided

or

$$
\begin{gathered}
f^{\prime}\left(\frac{\partial \xi}{\partial x}\right)= \pm c /\left(1+\frac{\partial \xi}{\partial x}\right), \ldots \\
\frac{\partial \xi}{\partial t}=f\left(\frac{\partial \xi}{\partial x}\right)= \pm c \log \left(1+\frac{\partial \xi}{\partial x}\right),
\end{gathered}
$$


no additive constant being necessary if we assume that $\dot{\xi}=0$ in the parts of the medium not affected by the wave. This may also be written

$$
\dot{\xi}=\mp c \log (1+s)
$$

by $\S 59$ (3). Another form is

$$
\rho / \rho_{0}=e^{\mp \dot{\xi} / c} \text {. }
$$

When $s$ is infinitesimal the formula (16) reduces to $\dot{\xi}=\mp c s$, in agreement with $\S 60$.

To find the rate at which any particular value of $s$ is propagated, in either of these cases, we note that the value of $\partial \xi / \partial x$ which is associated with the particle $x$ at the instant $t$ will have been transmitted to the particle $x+\delta x$ at the instant $t+\delta t$, provided

i.e. by (12) and (14),

$$
\frac{\partial^{2} \xi}{\partial x \partial t} \delta t+\frac{\partial^{2} \xi}{\partial x^{2}} \delta x=0,
$$

$$
\delta x \pm c(1+s) \delta t=0 .
$$

The phase $s$ is therefore propagated with the velocity

$$
\frac{d x}{d t}=\mp c(1+s)
$$

relative to the undisturbed medium. To find the rate of propagation in space we have to take account of the total variation of $x+\xi$, which is

$$
\left(1+\frac{\partial \xi}{\partial x}\right) \delta x+\frac{\partial \xi}{\partial t} \delta t
$$

The required velocity is therefore

$$
\left(1+\frac{\partial \xi}{\partial x}\right) \frac{d x}{d t}+\frac{\partial \xi}{\partial t}=\mp c+\frac{\partial \xi}{\partial t} .
$$

The lower sign relates to a wave travelling in the direction of $x$-positive. It appears from (16) that positive values of $\dot{\xi}$ are then associated with positive values of $s$, as in the approximate theory of $\S 60$; but the formula (20) shews that the velocity of propagation is greater, the greater the value of $s$. The parts of the wave where the density is greater therefore gain continually on those where it is less. Thus if the relation between $s$ and $x$ be exhibited graphically, the curve $A$ in the annexed 
figure takes after a time some such form as $B^{*}$. The wave becomes, so to speak, continually steeper in front, and slopes more gradually in the rear, until a time arrives at which the gradient at some point becomes infinite. After this stage the analysis ceases to have any real meaning.
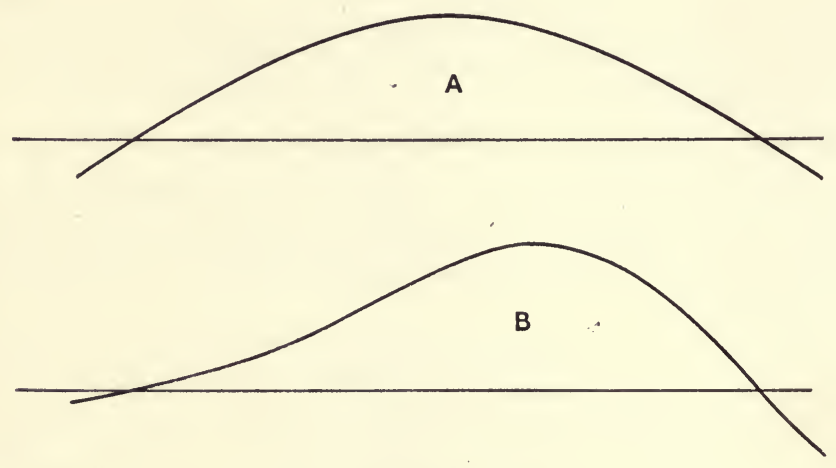

Fig. 62.

The adiabatic hypothesis leads to results of the same general character. The reader will find no difficulty in verifying the following statement. The formula (16) is replaced by

$$
\frac{\partial \xi}{\partial t}= \pm \frac{2 c}{\gamma-1}\left\{1-(1+s)^{\frac{1}{2}(\gamma-1)}\right\}
$$

and the velocity of propagation of a particular value of $s$ is

$$
\mp c(1+s)^{\frac{1}{2}(\gamma+1)}
$$

relative to the undisturbed medium, or

$$
\mp\left(\frac{d p}{d \rho}\right)^{\frac{1}{2}}+\frac{\partial \xi}{\partial t}
$$

in space. In the latter formula the particle-velocity is added to the velocity of sound proper to the actual density, which is on the adiabatic hypothesis dependent on the degree of condensation and consequent change of temperature. The general conclusions are as before.

* It is not very important here whether the coordinate $x$ be supposed (as in the previous part of this investigation) to refer to the undisturbed medium, or to be an ordinary space-coordinate. In either case the tendency is the same. 
It must be remembered that since the equation of motion (9) is not linear, distinct solutions, such as those representing waves travelling right and left, respectively, which we have just been considering, cannot be superposed by mere addition. It may however be remarked that, as a result of a more complete investigation, Riemann* found (1860) that a localized arbitrary initial disturbance does eventually resolve itself into two waves of the above kinds, travelling in opposite directions.

To follow exactly the career of waves of finite amplitude generated in any given manner is a problem of considerable difficulty; but some indications may be obtained by methods of approximation. This procedure was adopted by Airy $†(1845)$ in his work on the dynamical theory of the tides, where similar questions arise with respect to tides in shallow seas and estuaries.

Suppose, for instance, we have a long straight tube in which a piston (at $x=0$ ) is made to move in an arbitrary manner

$$
\xi=f(t) \text {. }
$$

The equation (9) becomes, if we neglect terms of the third order in the derivatives of $\xi$,

$$
\frac{\partial^{2} \xi}{\partial t^{2}}=c^{2} \frac{\partial^{2} \xi}{\partial x^{2}}-(\gamma+1) c^{2} \frac{\partial \xi}{\partial x} \frac{\partial^{2} \xi}{\partial x^{2}} .
$$

If we omit the last term, we have as in $\S 60$ the first approximation

$$
\xi=f\left(t-\frac{x}{c}\right)
$$

Substituting this value of $\xi$ in the small term of (25) we obtain

$$
\frac{\partial^{2} \xi}{\partial t^{2}}=c^{2} \frac{\partial^{2} \xi}{\partial x^{2}}-\frac{1}{2}(\gamma+1) \frac{\partial}{\partial x}\left\{f^{\prime}\left(t-\frac{x}{c}\right)\right\}^{2} .
$$

The solution of this which is consistent with (26) is

$$
\xi=f\left(t-\frac{x}{c}\right)+\frac{\gamma+1}{4 c^{2}} x\left\{f^{\prime}\left(t-\frac{x}{c}\right)\right\}^{2},
$$

as is easily verified. The correction to the first approximation

* Bernhard Riemann (1826-66), professor of mathematics at Göttingen $1857-66$.

+ Sir George Biddell Airy (1801-92), Plumian professor of astronomy at Cambridge 1828-35, astronomer royal $1835-81$. 
(26) is proportional to $x$, and to the square of the ratio of the velocity of the piston to the velocity of sound. This latter ratio may in practice be exceedingly small, but as we travel to the right the correction continually increases in importance, until at length the neglect of terms of the third and higher orders would no longer be justified. This is what we should expect from the results of Earnshaw's investigation.

When the motion of the piston is simple-harmonic, say

the formula (28) gives

$$
f(t)=a \cos n t
$$

$$
\xi=a \cos n\left(t-\frac{x}{c}\right)+\frac{(\gamma+1) n^{2} a^{2}}{8 c^{2}} x\left\{1-\cos 2 n\left(t-\frac{x}{c}\right)\right\} .
$$

The displacement of any particle is no longer simple-harmonic, but consists of a part independent of $t$ together with two simple-harmonic terms, one having the frequency of the imposed vibration (29), and the other a frequency twice as great. This illustrates the implied limitation to infinitely small motions in the usual theory of forced oscillations ( $\$ 17)$.

Again, if the given vibration of the piston be made up of two simple-harmonic components, say

we find

$$
f(t)=a_{1} \cos n_{1} t+a_{2} \cos n_{2} t
$$

$$
\begin{aligned}
\xi=a_{1} \cos n_{1}\left(t-\frac{x}{c}\right)+a_{2} \cos n_{2}\left(t-\frac{x}{c}\right) \\
+\frac{\gamma+1}{8 c^{2}} x\left\{n_{1}^{2} a_{1}^{2}+n_{2}^{2} a_{2}^{2}-n_{1}{ }^{2} a_{1}^{2} \cos 2 n_{1}\left(t-\frac{x}{c}\right)\right. \\
-n_{2}{ }^{2} a_{2}^{2} \cos 2 n_{2}\left(t-\frac{x}{c}\right) \\
+2 n_{1} n_{2} a_{1} a_{2} \cos \left(n_{1}-n_{2}\right)\left(t-\frac{x}{c}\right) \\
\left.-2 n_{1} n_{2} a_{1} a_{2} \cos \left(n_{1}+n_{2}\right)\left(t-\frac{x}{c}\right)\right\} .
\end{aligned}
$$

We thus learn that in addition to the vibrations of double frequency, other simple-harmonic vibrations whose frequencies are respectively the difference and the sum of the primary frequencies now make their appearance. In acoustical language, two simple vibrations of sufficient amplitude may give rise not 
only to the corresponding pure tones, but to their octaves, as well as to certain "combination-tones," whose occurrence reminds us again, that the principle of superposition is no longer valid. We shall have occasion to refer to this investigation at a later period (Chap. X).

The analogous phenomenon in tidal theory is the production of "over-tides," which are in fact appreciable, and have to be provided for in the Harmonic Analysis referred to in $§ 39$.

We have seen that the main effect of finite amplitude is that in a progressive wave the gradients, both of pressure and of density, tend to become infinite. This has suggested the question whether a wave of discontinuity might not finally be established, analogous to a "bore" in water-waves. To examine into the possibility of such a wave we take the question in its simplest form, and assume that the circumstances are everywhere uniform, except for the sudden transition at the plane of discontinuity. Further, by the superposition of a certain uniform velocity, we reduce the problem to one of steady motion in which the plane in question is fixed.

The symbols $p_{0}, \rho_{0}, u_{0}$ will then be supposed to refer to the region to the left of this plane, whilst the values of the corresponding quantities on the right are denoted by $p, \rho, u$. Since in every unit of time the same mass $(m)$ of fluid crosses any unit area normal

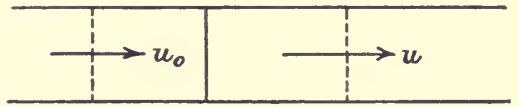

Fig. 63. to the direction of flow, we have

$$
\rho u=\rho_{0} u_{0}=m \text {, or } u=m v, u_{0}=m v_{0} .
$$

Again, since in unit time a mass $m$ has its velocity changed from $u_{0}$ to $u$, the momentum of the portion of air included between two planes in the positions indicated by the dotted lines in Fig. 63 is increasing at the rate $m\left(u-u_{0}\right)$, whence

or, in virtue of (3:3),

$$
p_{0}-p=m\left(\iota-u_{0}\right),
$$

$$
p_{0}-p=m^{2}\left(v-v_{0}\right),
$$

in agreement with (5). If we now superpose a uniform velocity 
$-u_{0}$, we get the case of a wave advancing into a region previously at rest. The wave-velocity is given by

$$
u_{0}^{2}=m^{2} v_{0}^{2}=\frac{p-p_{0}}{v_{0}-v} v_{0}^{2}=\frac{p-p_{0}}{\rho-\rho_{0}} \cdot \frac{\rho}{\rho_{0}},
$$

as first found by Stokes (1848), and afterwards independently by Earnshaw, Riemann, and Rankine. A difficulty, first pointed out by Lord Rayleigh, arises, however, as to the conservation of energy. The rate at which work is being done on the portion of air above considered is $p_{0} u_{0}-p u$, whilst that at which the kinetic energy is increasing is $\frac{1}{2} m\left(u^{2}-u_{0}^{2}\right)$. The difference is

$$
p_{0} u_{0}-p u-\frac{1}{2} m\left(u^{2}-u_{0}^{2}\right)=\frac{1}{2} m\left(p_{0}+p\right)\left(v_{0}-v\right) . \ldots
$$

If the two points $(v, p),\left(v_{0}, p_{0}\right)$ on the indicator diagram be denoted by $P, P_{0}$, respectively, the expression (37) is $m$ times the area of the trapezium bounded by the straight line $P_{0} P$, the axis of $v$, and the ordinates $p_{0}, p$. If the transition be effected without gain or loss of heat, the points $P_{0}, P$ will lie on the same adiabatic, and the gain of intrinsic energy will be represented by the area included between this curve, the axis of $v$, and the same two ordinates. Since the adiabatics are concave upwards, the latter area is (in absolute value) less than the former. It appears on examination of the signs to be attributed to the areas that if $v_{0}>v$ the work done is more than is accounted for by the increase of the kinetic and intrinsic energies, whilst if $v_{0}<v$ the work given out would be more than is equivalent to the apparent loss of energy.

It is evident that no complete theory of waves of discontinuity can be attempted without some reference to viscosity and to thermal conduction, since at the point of transition the gradients of velocity and temperature are infinite.

It does not appear probable that under ordinary conditions the modifications due to finite amplitude are of serious importance. In equation (30), for instance, the ratio of the amplitude of the vibration of the second order to that of the primary vibration is comparable with $n^{2} a x / c^{2}$, or with $n^{2} a / g . x / H$, where $H$ is the height of the homogeneous atmosphere. With ordinary amplitudes $a$, and ordinary distances $x$, this ratio will be very small. In three dimensions the effect must be very 
much less, owing to the diminution of amplitude by spherical divergence.

\section{Viscosity.}

The essence of viscosity is that in a moving fluid the stresses differ from a state of pressure uniform in all directions about a point, by quantities depending on the rates of deformation. It is usually assumed that these quantities are linear functions of the rates of strain; from our present standpoint this is sufficiently justified by the fact that the strain-velocities are regarded as infinitely small. As in $\$ 40$ there will at any instant, and at any given point, be three principal axes of the deformation which is taking place, and these will naturally be the principal axes of the corresponding stress. We therefore write, by analogy with $§ 42(1)$,

$$
\left.\begin{array}{l}
p_{1}=-p+\lambda^{\prime} \dot{\Delta}+2 \mu^{\prime} \dot{\boldsymbol{\epsilon}}_{1}, \\
p_{2}=-p+\lambda^{\prime} \dot{\Delta}+2 \mu^{\prime} \dot{\boldsymbol{\epsilon}}_{2}, \\
p_{3}=-p+\lambda^{\prime} \dot{\Delta}+2 \mu^{\prime} \dot{\boldsymbol{\epsilon}}_{3},
\end{array}\right\}
$$

where $\dot{\epsilon}_{1}, \dot{\epsilon}_{2}, \dot{\epsilon}_{3}$ are the principal strain-velocities, and

$$
\dot{\Delta}=\dot{\epsilon}_{1}+\dot{\epsilon}_{2}+\dot{\epsilon}_{3} \text {. }
$$

By the same kind of proof as in $\S 41, \mu^{\prime}$ is recognized as the coefficient of viscous resistance to a shearing motion in parallel planes; viz. if $\dot{\eta}$ denote the rate of shear, and $\varpi$ the corresponding stress, we have

$$
\varpi=\mu^{\prime} \dot{\eta}
$$

The value of $\mu^{\prime}$ has been determined with considerable accuracy for a number of fluids, gaseous as well as liquid.

It will be noticed that the meaning of the symbol $p$, and consequently the value of $\lambda^{\prime}$, is so far indeterminate, since nothing is altered in the shape of the formulae (1) if we incorporate in $p$ any constant multiple of $\dot{\Delta}$. In the case of liquids it is in fact usual so to incorporate the second terms in (1). In the application to gases it is convenient to regard $p$ as defined by the gaseous laws $(p=R \rho \theta)$. There is at present no experimental evidence as to how far the mean stress about a point, viz.

$$
\frac{1}{3}\left(p_{1}+p_{2}+p_{3}\right)=-p+\left(\lambda^{\prime}+\frac{2}{3} \mu^{\prime}\right) \dot{\Delta}
$$


differs, in a moving gas, from $-p$, as thus fixed; but from considerations based on the kinetic theory of gases Maxwell* inferred (1866) that the two things are identical, and that accordingly

$$
\lambda^{\prime}=-\frac{2}{3} \mu^{\prime}
$$

As we are interested chiefly in the order of magnitude of the effects, the precise determination of $\lambda^{\prime}$ is not of much consequence to us; accordingly Maxwell's view is adopted for simplicity in what follows.

The dimensions of $\mu^{\prime}$ are those of a stress multiplied by a time, or $\left[M L^{-1} T^{-1}\right]$. It is found that $\mu^{\prime}$ is independent of the density, but (in gases) increases with rise of temperature. Its value for air at $0^{\circ} \mathrm{C}$. is about 000170 in absolute c.G.S. units. It will appear however immediately that the effect of viscosity in modifying motion depends not so much on the value of $\mu^{\prime}$ as on its ratio to the inertia of the fluid. This ratio

$$
\nu=\mu^{\prime} / \rho_{0}
$$

is therefore called by Maxwell the "kinematic" coefficient of viscosity; its dimensions are $\left[L^{2} T^{-1}\right] . \quad$ For air at $0^{\circ} \mathrm{C}$. its value is about $\cdot 132$ C.G.S.

The rate at which the stresses on the faces of a unit cube are doing work in changing its size and shape is given by

$$
\begin{aligned}
p_{1} \dot{\epsilon}_{1}+p_{2} \dot{\epsilon}_{2} & +p_{3} \dot{\epsilon}_{3}=-p \dot{\Delta}+\lambda^{\prime} \dot{\Delta}^{2}+2 \mu^{\prime}\left(\dot{\epsilon}_{1}^{2}+\dot{\epsilon}_{2}^{2}+\dot{\epsilon}_{3}^{2}\right) \\
& =-p \dot{\Delta}+\frac{2}{3} \mu^{\prime}\left\{\left(\dot{\epsilon}_{2}-\dot{\epsilon}_{3}\right)^{2}+\left(\dot{\epsilon}_{3}-\dot{\epsilon}_{1}\right)^{2}+\left(\dot{\epsilon}_{1}-\dot{\epsilon}_{2}\right)^{2}\right\} .
\end{aligned}
$$

The term $-p \dot{\Delta}$ represents the rate at which the intrinsic energy is increasing. The remaining terms, which are essentially positive, indicate a dissipation of energy at the rate

$$
\frac{2}{3} \mu^{\prime}\left\{\left(\dot{\epsilon}_{2}-\dot{\epsilon}_{3}\right)^{2}+\left(\dot{\epsilon}_{3}-\dot{\epsilon}_{1}\right)^{2}+\left(\dot{\epsilon}_{1}-\dot{\epsilon}_{2}\right)^{2}\right\}
$$

per unit volume. The mechanical energy thus lost is converted into heat. It will be noticed that $(7)$ vanishes in the case of uniform expansion $\left(\dot{\epsilon}_{1}=\dot{\epsilon}_{2}=\dot{\epsilon}_{3}\right)$; this is a necessary consequence of our previous assumption as to the value of the constant $\lambda^{\prime}$.

* James Clerk Maxwell (1831-79), professor of experimental physics at Cambridge (1871-79); author of the electromagnetic theory of light. 
In the case of a pure shearing motion $(\dot{\eta})$, the formula (6) takes the shape

$$
\varpi \dot{\eta}=\mu^{\prime} \dot{\eta}^{2}
$$

In plane waves of sound we have $\dot{\epsilon}_{2}=0, \dot{\epsilon}_{3}=0$, and therefore from (1) and (4)

$$
p_{1}=-p+\frac{4}{3} \mu^{\prime} \dot{\epsilon}_{1}=-p_{0}-\kappa s+\frac{4}{3} \mu^{\prime} \dot{\epsilon}_{1} .
$$

Moreover, in the notation of $\S 59$,

$$
s=-\frac{\partial \xi}{\partial x}, \quad \dot{\epsilon}_{1}=\frac{\partial}{\partial t}\left(\frac{\partial \xi}{\partial x}\right)
$$

The equation of motion, viz.

therefore becomes

$$
\rho_{0} \frac{\partial^{2} \xi}{\partial t^{2}}=\frac{\partial p_{1}}{\partial x}
$$

$$
\frac{\partial^{2} \xi}{\partial t^{2}}=c^{2} \frac{\partial^{2} \xi}{\partial x^{2}}+\frac{4}{3} \nu \frac{\partial^{3} \xi}{\partial x^{2} \partial t} .
$$

To obtain a solution appropriate to the case of free waves we put

$$
\xi=P \cos k x,
$$

where $P$ is a function of $t$, to be determined. We find that (12) will be satisfied, provided

$$
\frac{d^{2} P}{d t^{2}}+\frac{4}{3} \nu k^{2} \frac{d P}{d t}+k^{2} c^{2} P=0 .
$$

This has the form of $\S 11(3)$, and the solution is therefore

$$
P=C e^{-t / \tau} \cos (n t+\epsilon)
$$

provided

$$
\tau=3 / 2 \nu k^{2}, \quad n^{2}=k^{2} c^{2}-1 / \tau^{2} .
$$

In all cases of interest $c \tau$ is a considerable multiple of the wave-length $(\lambda=2 \pi / k)$, so that $n=k c$, practically, the friction having as usual no appreciable effect on the period. Thus

$$
\xi=C e^{-t / \tau} \cos (k c t+\epsilon) \cdot \cos k x \text {. }
$$

This represents a system of standing waves with fixed nodes and loops. There is a similar solution in which $\cos k x$ is replaced by $\sin k x$, and by superposition of the two we can construct a progressive wave-system

$$
\xi=C e^{-t / \tau} \cos k(c t-x) \text {. }
$$

Putting $\nu=\cdot 132$ for the case of air, we find $\tau=\cdot 288 \lambda^{2}$, the units being the second and the centimetre. 
The solution of (14) may also be effected concisely by means of imaginary quantities. Thus in investigating forced simpleharmonic vibrations of prescribed frequency we assume that

$$
\xi=a e^{i n t+m x},
$$

whence, on substitution,

$$
m^{2}=-\frac{n^{2}}{c^{2}+\frac{4}{3} i \nu n} \text {. }
$$

The ratio $\nu n / c^{2}$ is usually very small; thus for $n=1500$ its value is, with previous data, about $1.8 \times 10^{-7}$. Hence

$$
m= \pm \frac{i n}{c}\left(1-\frac{2}{3} \frac{i \nu n}{c^{2}}\right)
$$

Taking the lower sign, which corresponds to waves travelling in the direction of $x$-positive, and rejecting the imaginary part of (19), we find

$$
\xi=a e^{-x / l} \cos n\left(t-\frac{x}{c}\right),
$$

provided

$$
l=3 c^{3} / 2 \nu n^{2} \text {. }
$$

This represents a system of waves generated to the right of the origin by a prescribed motion $\xi=a \cos n t$ at this point (as by a piston in a tube if we neglect the friction at the sides). The waves advance, with (sensibly) the usual velocity $c$, but diminish exponentially in amplitude as they proceed*. The linear magnitude $l$ measures the distance over which the waves travel before the amplitude is diminished in the ratio $1 / e$. In terms of the wave-length we have

$$
l=\left(3 c / 8 \pi^{2} \nu\right) \cdot \lambda^{2},
$$

or, with previous data, $l=9.56 \lambda^{2} \times 10^{3}$. The effect of viscosity in stifling the vibrations is therefore very slight except in the case of sounds of very high frequency and consequently short wave-length. Even for $\lambda=10 \mathrm{~cm}$. the value of $l$ is nearly 10 kilometres. When we come to the discussion of threedimensional waves it will be clear that the effect of viscosity may for most purposes be ignored in comparison with the diminution of intensity due to spherical divergence. It is, however, of some interest to observe that there is a physical

* This calculation was first made by Stokes (1845). 
limit to the frequency of vibrations which are capable of propagation for more than a very moderate distance.

The viscosity being small, the rate at which work is done per unit area by the piston in maintaining the wave-system (22) must have sensibly the value $\frac{1}{2} \rho_{0} n^{2} a^{2} c$ found in $\S 60$. Since the energy in the medium to the right is now finite and on the average constant, this must be equal to the rate of dissipation of energy by viscosity. The equality is easily verified. The dissipation is, by (7),

$$
\begin{aligned}
\frac{4}{3} \mu^{\prime} \int_{0}^{\infty} \dot{\epsilon}_{1}^{2} d x & =\frac{4}{3} \mu^{\prime} \int_{0}^{\infty}\left(\frac{\partial^{2} \xi}{\partial x \partial t}\right)^{2} d x \\
& =\frac{4}{3} \mu^{\prime} \frac{n^{4} a^{2}}{c^{2}} \int_{0}^{\infty} e^{-2 x / l} \cos ^{2} n\left(t-\frac{x}{c}\right) d x, \ldots
\end{aligned}
$$

approximately, if we keep only the most important term. Writing

$$
\cos ^{2} n\left(t-\frac{x}{c}\right)=\frac{1}{2}+\frac{1}{2} \cos 2 n\left(t-\frac{x}{c}\right),
$$

and taking the mean value with respect to the time, we obtain

by (23).

$$
\frac{4}{3} \mu^{\prime} \frac{n^{4} a^{2}}{c^{2}} \cdot \frac{1}{4} l=\frac{1}{2} \rho_{0} n^{2} a^{2} c,
$$

\section{Effect of Heat Conduction.}

A further cause of dissipation of energy is to be found in the thermal processes consequent on the alternate expansions and rarefactions of the air. If indeed these succeed each other with sufficient rapidity, the variations are almost accurately adiabatic, as explained in $\S 59$; but, as was first pointed out by Kirchhoff (1868), the residual conduction of heat is in any case of equal importance with viscosity. On the kinetic theory of gases the coefficients of "thermometric" conductivity $\left(\nu^{\prime}\right)$ and of kinematic viscosity are in fact of the same order of magnitude; according to Maxwell the relation is $\nu^{\prime}=\frac{5}{2} \nu$. For this reason the preceding calculations of the effect of viscosity on air-waves must not be looked upon as more than illustrative. A complete investigation, in which both influences are taken into account, shews that the effect is equivalent to an increase in the kinematic viscosity, but the order of magnitude is unaffected. 
If on the other hand the alternations of density were to take place with extreme slowness, as in the case of very long waves of simple-harmonic type, there would be time for practical equalization of temperature, and the dissipative influence of conduction as well as viscosity would again be insignificant. Since the expansions are here nearly isothermal, the wave-velocity will approximate to the Newtonian value $(\$ 59(10))$.

In intermediate cases the theory shews that the wavevelocity would no longer be constant, but perceptibly dependent on the frequency. Since no such effect is observed, we infer that in all ordinary cases the conditions are practically adiabatic. It appears also that in such intermediate cases the dissipation would be very greatly increased. The investigation of Stokes (1851), which is here referred to, relates to the effect of radiation; the extension to conduction was made independently by Kirchhoff and Lord Rayleigh. It is probable that the effects of radiation alone are of subordinate importance.

The detailed calculation must be passed over, but the general explanation of the manner in which thermal processes may operate to produce dissipation of energy has been stated with such admirable clearness by Stokes that it is worth while to reproduce the passage in question. The explicit reference is to radiation, but the same principles are involved in the case of conduction also.

"Conceive a mass of air contained in a cylinder in which an air-tight piston fits, which is capable of moving without friction, and which has its outer face exposed to a constant atmospheric pressure; and suppose the air alternately compressed and rarefied by the motion of the piston. If the motion take place with extreme slowness, there will be no sensible change of temperature, and therefore the work done on the air during compression will be given out again by the air during expansion, inasmuch as the pressure on the piston will be the same when the piston is at the same point of the cylinder, whether it be moving forwards or backwards. Similarly, the work done in rarefying the air will be given out again by the atmosphere as the piston returns towards its position of equilibrium, so that 
the motion would go on without any permanent consumption of labouring force. Next, suppose the motion of the piston somewhat quicker, so that there is a sensible change of temperature produced by condensation and rarefaction. As the piston moves forward in condensing the air, the temperature rises, and therefore the piston has to work against a pressure greater than if there had been no variation of temperature. By the time the piston returns, a good portion of the heat developed by compression has passed off, and therefore the piston is not helped as much in its backward motion by the pressure of the air in the cylinder as it had been opposed in its forward motion. Similarly, as the piston continues its backward motion, rarefying the air, the temperature falls, the pressure of the air in the cylinder is diminished more than corresponds merely to the change of density, and therefore the piston is less helped in opposing the atmospheric pressure than it would have been had the temperature remained constant. But by the time the piston is returning towards its position of equilibrium, the cold has diminished in consequence of the supply of heat from the sides of the cylinder, and therefore the force urging the piston forward, arising, as it does, from the excess of the external over the internal pressure, is less than that which opposed the piston in moving from its position of equilibrium. Hence in this case the motion of the piston could not be kept up without a continual supply of labouring force. Lastly, suppose the piston to oscillate with great rapidity, so that there is not time for any sensible quantity of heat to pass and repass between the air and the sides of the cylinder. In this case the pressures would be equal when the piston was at a given point of the cylinder, whether it were going or returning, and consequently there would be no permanent consumption of labouring force. I do not speak of the disturbance of the external air, because I am not now taking into account the inertia of the air either within or without the cylinder. The third case, then, is similar to the first, so far as regards the permanence of the motion; but there is this difference; that, in consequence of the heat produced by compression and the cold produced by rarefaction, the force urging the piston towards its position of equilibrium, on 
whichever side of that position the piston may happen to be, is greater than it would have been had the temperature remained unaltered.

"Now the first case is analogous to that of the sonorous vibrations of air when the heat and cold produced by sudden condensation and rarefaction are supposed to pass away with great rapidity. For we are evidently concerned only with the relative rates at which the phase of vibration changes, and the heat causing the excess of temperature $\theta$ passes away, so that it is perfectly immaterial whether we suppose the change of motion to be very slow, or the cooling of heated air to be very rapid. The second case is analogous to that of sound, when we suppose the constants $q^{*}$ and $n$ comparable with each other; and we thus see how it is, that, on such a supposition, labouring force would be so rapidly consumed, and the sound so rapidly stifled. The third case is analogous to that of sound when we make the usual supposition, that the alternations of condensation and rarefaction take place with too great rapidity to allow a given portion of air to acquire or lose any sensible portion of heat by radiation. The increase in the force of restitution of the piston, arising from the alternate elevation and depression of temperature, is analogous to the increase in the forces of restitution of the particles of air arising from the same cause, to which corresponds an increase in the velocity of propagation of sound."

\section{Damping of Waves in Narrow Tubes and Crevices.}

A somewhat greater effect of viscosity may be looked for when the air is in contact with a solid body, as at the walls of a pipe or resonator, owing to the practically infinite resistance which the surface opposes to the sliding of the fluid immediately in contact with it. It seems in fact to be well-established that the relative velocity vanishes at the surface, whereas in our theoretical investigations we assume for the most part that sliding takes place quite freely. A closer examination shews however that in the case of rapid vibrations, such as we are concerned with in acoustics, the effect is mainly local, being

* $[q$ is a constant of radiation. $]$ 
confined, practically, to a very thin layer of air near the surface, and is except in very narrow spaces unimportant.

The matter may be sufficiently illustrated by a very simple case. Suppose that the fluid above the plane $y=0$ is acted on by a periodic force

$$
X=f \cos n t,
$$

per unit mass, parallel to $O x$, the plane forming a rigid boundary. The consequent motion being everywhere parallel to $O x$ and independent of the coordinate $x$, there is no variation of density, and the deformations which are taking place are of the nature of shearing motions parallel to $y=0$. Denoting the velocity $\dot{\xi}$ by $u$, the rate of shear will be

$$
\frac{\partial}{\partial t}\left(\frac{\partial \xi}{\partial y}\right)=\frac{\partial u}{\partial y}
$$

and the shearing stress on a plane parallel to $y=0$ is accordingly $\mu \partial u / \partial y$. The stratum bounded by the planes $y$ and $y+\delta y$ therefore experiences a resultant force

$$
\frac{\partial}{\partial y}\left(\mu^{\prime} \frac{\partial u}{\partial y}\right) \delta y
$$

per unit area, parallel to $x$, and the equation of motion is of the form

$$
\frac{\partial u}{\partial t}=\nu \frac{\partial^{2} u}{\partial y^{2}}+X .
$$

We have to solve this under the condition that $u=0$ for $y=0$. For conciseness we put $X=f e^{i n t}$, and reject (in the end) the imaginary part of our expressions. The equation is then satisfied by

$$
u=\left(\frac{f}{i n}+A e^{m y}\right) e^{i n t},
$$

provided $m^{2}=i n / \nu$, or

$$
m= \pm(1+i) \beta \text {, }
$$

where

$$
\beta=\sqrt{ }(n / 2 \nu) \text {. }
$$

Since we are looking for a solution which shall be finite for $y=\infty$ we take the lower sign. Also, the condition that $u=0$ for $y=0$ requires that $A=-f /$ in. Hence

$$
u=-\frac{i f}{n}\left\{1-e^{-(1+i) \beta y}\right\} e^{i n t},
$$


or, keeping only the real part,

$$
u=\frac{f}{n} \sin n t-\frac{f}{n} e^{-\beta y} \sin (n t-\beta y),
$$

a result which is easily verified. When $\beta y$ is moderately large the value of $u$ reduces practically to the first term, which is the same as if there had been no friction. The rigid boundary accordingly acts as a drag only on a thin stratum; for example when $y=2 \pi / \beta$ the velocity falls short of its value at a great distance from the surface by about one part in 535 .

In actual problems of acoustics (relating for example to vibrations in pipes) the force $\rho X$ per unit volume is replaced by the negative pressure-gradient $-\partial p / \partial x$, and we have of course changes of density to take into account, but the results have a similar interpretation. The linear magnitude

$$
h=2 \pi / \beta=\sqrt{ }(4 \pi \nu .2 \pi / n)
$$

may be taken to measure the extent to which the dragging effect penetrates into the fluid. With the previous data its value in centimetres is about $1 \cdot 29 / N^{\frac{1}{2}}$, where $N$ is the frequency; thus for $N=256$ we find $h=\cdot 80 \mathrm{~mm}$.

We may apply the above investigation to obtain an estimate of the effect of viscosity on the wave-velocity in a tube, on the supposition that the diameter is small compared with the wave-length but large compared with the quantity $h$. The tangential stress on the fluid at the boundary $y=0$ is, in the case of (7),

$$
-\mu^{\prime} \frac{\partial u}{\partial y}=-(1-i) \frac{\mu^{\prime} \beta f}{n}=-\frac{1-i}{4 \pi} h \rho f,
$$

by (9), the time-factor $e^{i n t}$ being understood. The total tangential force exerted by the walls of a cylindrical tube of radius $a$ on the contained air may therefore be equated to

$$
\frac{1}{2}(1-i) h a . \partial \bar{p} / \partial x
$$

per unit length, where $\bar{p}$ denotes the mean pressure over the section $\left(\pi a^{2}\right)$. Hence if $\bar{u}$ be the mean velocity, we have, calculating the forces on the air contained in an element $\delta x$ of the length,

$$
\pi \rho_{0} a^{2} \frac{\partial \bar{u}}{\partial t}=-\pi a^{2} \frac{\partial \bar{p}}{\partial x}+\frac{1}{2}(1-i) h a \frac{\partial \bar{p}}{\partial x},
$$


or

$$
\frac{\partial \bar{u}}{\partial t}=-\frac{1}{\rho_{0}} \frac{\partial \bar{p}}{\partial x}\left\{1-(1-i) \frac{h}{2 \pi a}\right\} \text {. }
$$

To this we must add the relations

$$
\begin{aligned}
& \bar{p}=p_{0}+c^{2} \rho_{0} \bar{s}, \\
& \frac{\partial \bar{s}}{\partial t}=-\frac{\partial \bar{u}}{\partial x} .
\end{aligned}
$$

The elimination of $\bar{p}$ and $\bar{s}$ between these equations leads to

$$
\frac{\partial^{2} \bar{u}}{\partial t^{2}}=\left\{1-(1-i) \frac{h}{2 \pi a}\right\} c^{2} \frac{\partial^{2} \bar{u}}{\partial x^{2}} .
$$

It is already assumed that the time enters through a factor $e^{i n t}$; and the solution of (14) is therefore of the type

$$
\bar{u}=C e^{i n t+m x},
$$

with

$$
\begin{aligned}
& m^{2}=-\frac{n^{2}}{c^{2}}\left\{1-(1-i) \frac{h}{2 \pi a}\right\}^{-1}, \\
& m= \pm \frac{i n}{c}\left\{1+(1-i) \frac{h}{4 \pi a}\right\},
\end{aligned}
$$

approximately, on account of the assumed smallness of $h / a$. For waves propagated in the direction of $x$-positive we take the lower sign, and write

where

$$
\begin{gathered}
m=-i n / c^{\prime}-\alpha, \\
c^{\prime}=\left(1-\frac{h}{4 \pi a}\right) c, \\
\alpha=n h / 4 \pi a c .
\end{gathered}
$$

and

We have, then

or, in real form, $\quad \bar{u}=C e^{-a x} \cos n\left(t-\frac{x}{c^{\prime}}\right)$.

The wave-velocity is therefore diminished in the ratio given by (19). The exponential factor in (22) expresses the law of decay of the waves as they advance. If $l$ be defined as in $\S 64$ (23) it will be found that $a l$ is of the order $\lambda^{2} / a h$. The rate of decay is therefore much greater under the present conditions than in the case of sound waves in the open.

A formula equivalent to (19) was published without demonstration by Helmholtz in 1863 . The above proof is a variation of that given by Lord Rayleigh in his Theory of Sound. 
A more complete investigation was instituted by Kirchhoff (1868) in which thermal processes are considered, as well as viscosity. The effects are thereby increased, as already explained, but remain of the same order of magnitude.

As already stated, it is implied in the above calculation that the diameter of the tube greatly exceeds the quantity $h$. When on the other hand the diameter is comparable with, or less than $h$, the walls have relatively a much greater hold on the vibrating mass, and the character of the motion is entirely altered by the friction. In particular, when $h$ is large compared with the width the mere inertia of the fluid ceases to have any appreciable influence, the mean velocity over a cross-section being determined by an approximately statical equilibrium between the pressure-gradient (in the direction of the length) and the friction of the walls. We have, then,

$$
R \bar{u}=-\frac{\partial \bar{p}}{\partial x}
$$

where $R$ is a coefficient of resistance, depending on the nature of the fluid, and on the shape and size of the cross-section. Again, by Boyle's law,

$$
\bar{p}=p_{0}(1+\bar{s})
$$

the isothermal hypothesis being adopted as now the most appropriate, since, owing to the assumed narrowness of the tube, transfer of heat can take place freely. Eliminating $\bar{p}$ and $\bar{s}$ between (13), (23), and (24), we find

$$
\frac{\partial \bar{u}}{d t}=\frac{p_{0}}{R} \frac{\partial^{2} \bar{u}}{\partial x^{2}} \text {. }
$$

This has the same form as the equation of linear conduction of heat. Assuming that

$$
\bar{u}=C e^{i n t+m x},
$$

we have $m^{2}=i n R / p_{0}$, and therefore

if

$$
\begin{gathered}
m= \pm(1+i) \varpi, \\
\varpi^{2}=\frac{1}{2} n R / p_{0} .
\end{gathered}
$$

Taking the lower sign we obtain

$$
\bar{u}=C e^{-\varpi x+i(n t-\varpi x)},
$$

or, in real form, $\quad \bar{u}=C e^{-\varpi x} \cos (n t-\varpi x)$. 
The value of $R$ will be sensibly the same as if the fluid were incompressible. Its determination is therefore the same as in the case of the steady flow of a liquid under pressure through a capillary tube. In this case, if the section be circular, the shearing stress per unit length on a coaxial cylindrical surface of radius $r$ is $2 \pi r \cdot \mu^{\prime} \partial u / \partial r$, and the resultant of the longitudinal forces on the two curved faces of a cylindrical shell of thickness $\delta r$ is therefore

$$
2 \pi \mu^{\prime} \frac{\partial}{\partial r}\left(r \frac{\partial u}{\partial r}\right) \delta r
$$

per unit length. The sectional area of the shell being $2 \pi r \partial r$, the requisite pressure-gradient is

$$
\frac{\partial p}{\partial x}=\frac{\mu^{\prime}}{r} \frac{\partial}{\partial r}\left(r \frac{\partial u}{d r}\right)
$$

which is independent of $x$. There being no radial motion, we have $\partial p / \partial r=0$, so that $p$, and therefure $\partial p / \partial x$, is also independent of $r$. The equation (31) is then satisfied by $u=A+B r^{2}$, provided $B$ be properly determined. The constant $A$ is fixed by the consideration that there is no slipping at the wall $(r=a)$ of the tube. In this way we find

$$
u=-\frac{\partial p}{\partial x} \cdot \frac{a^{2}-r^{2}}{4 \mu^{\prime}} \text {. }
$$

The mean velocity over the area of the section is therefore

$$
\bar{u}=\frac{1}{\pi a^{2}} \int_{0}^{a} u \cdot 2 \pi r d r=-\frac{\partial p}{\partial x} \cdot \frac{a^{2}}{8 \mu^{\prime}} .
$$

Hence, for a circular section,

$$
R=8 \mu^{\prime} / a^{2} \text {. }
$$

The formula (33) contains Poiseuille's* law of efflux of liquid through a capillary tube, viz. that the discharge per second varies as the pressure-gradient and as the fourth power of the diameter. It may be made the basis of an experimental method of determining $\mu^{\prime}$.

* J. L. M. Poiseuille (1799-1869), a practising physician in Paris, who was interested in the capillary circulation of the blood. The date of the memoir referred to is $\mathbf{1 8 4 4}$. 
The case of an elliptic section can be solved in a similar manner. The result, first given by Boussinesq (1868), is

$$
R=4 \mu^{\prime}\left(a^{2}+b^{2}\right) / a^{2} b^{2}
$$

where $a, b$ are the semi-axes. If we put $a=\infty$ we get the case of a narrow crevice, bounded by parallel planes, the breadth being $2 b$, viz.

$$
R=4 \mu^{\prime} / b^{2} .
$$

This can of course be obtained more easily by an independent process.

The formula (30), when combined with (34) or (36), agrees with the result of the more complete investigation given by Lord Rayleigh (1883). It appears that $\bar{u}$ goes through its cycle of phases in a distance $2 \pi / \varpi$, but that within this space the amplitude is diminished in the ratio $e^{-2 \pi}=1 / 535$. In the case of circular section we have

$$
\varpi^{2}=4 \mu^{\prime} n / p_{0} a^{2},
$$

by (28) and (34). Hence when the circumstances are such that the ratio $\nu / n a^{2}$ is large, the distance in question is small compared with the wave-length $(\lambda=2 \pi c / n)$ in the open; for we have

$$
(\lambda \varpi / 2 \pi)^{2}=\varpi^{2} c^{2} / n^{2}=4 \nu / n a^{2} .
$$

Hence in a sufficiently narrow tube the waves are rapidly stifled, the mechanical energy lost being of course converted into heat.

The investigation has been employed by Lord Rayleigh to illustrate the absorption of sound by porous bodies. When a sound-wave impinges on a slab which is permeated by a large number of very minute channels, part of the energy is lost, so far as sound is concerned, by dissipation within these channels, in the way just explained. The interstices in hangings and carpets act in a similar manner, and it is to this cause that the effect of such appliances in deadening echoes in a room is to be ascribed, a certain proportion of the energy being lost at each reflection. It is to be observed that it is only through the action of true dissipative forces, such as viscosity and thermal conduction, that sound can die out in an enclosed space, no mere modification of the waves by irregularities being of any avail. 


\section{CHAPTER VII}

\section{GENERAL THEORY OF SOUND WAVES}

\section{Definitions. Flux. Divergence.}

In respect of notation it is convenient now to take a point of view somewhat different from that adopted in the preceding chapter. We denote by $u, v, w$ the component velocities, parallel to rectangular axes, considered as functions of position $(x, y, z)$ and of time $t$. With each point of space there is accordingly associated, at any given instant, a vector $(u, v, w)$, and the whole assemblage of such vectors gives an instantaneous picture of the distribution of velocity*. On the other hand the variations of $u, v, w$ with the time, for given values of $x, y, z$, give the history of what goes on at a particular place $\dagger$, but supply in the first instance no information as to the careers of the various particles which (so to speak) successively cross the scene.

When we proceed to calculate the component accelerations of the particle which at the instant $t$ is in the position $(x, y, z)$ we have to take account of the fact that after the lapse of a time however short its velocities $u, v, w$ will be given by the respective functions of the altered position as well as the altered epoch. Suppose that at two successive instants $t_{1}, t_{2}$ a particle occupies the positions $P$ and $P^{\prime}$, respectively, and that the corresponding values of the $x$-component of the velocity are

* M. Marey and others have taken photographs, of short exposure, of a twodimensional current of water carrying suspended motes. The image of each mote is drawn out into a short line, which indicates the direction and magnitude of the corresponding velocity.

+ As if we were to view the surface of a stream through a narrow tube. 
$u_{1}, u_{2}$ at $P$ and $u_{1}^{\prime}, u_{2}^{\prime}$ at $P^{\prime}$. The $x$-component of the acceleration of this particle will be the limit of

$$
\frac{u_{2}^{\prime}-u_{1}}{t_{2}-t_{1}}=\frac{u_{2}-u_{1}}{t_{2}-t_{1}}+\frac{u_{2}^{\prime}-u_{2}}{t_{2}-t_{1}}
$$

The limit of the first term on the right is $\partial u / \partial t$, the rate of change of $u$ at $P$. Again $u_{2}^{\prime}-u_{2}$ is the difference of simultaneous velocities at the points $P, P^{\prime}$, so that, ultimately,

$$
u_{2}^{\prime}-u_{2}=\frac{\partial u}{\partial s} \cdot P P^{\prime}=\frac{\partial u}{\partial s} q\left(t_{2}-t_{1}\right),
$$

where $\partial u / \partial s$ is a space-differentiation in the direction $P P^{\prime}$, and $q$ is the resultant velocity $\sqrt{ }\left(u^{2}+v^{2}+w^{2}\right)$. The final expression for the acceleration parallel to $x$ is therefore

$$
\frac{\partial u}{\partial t}+q \frac{\partial u}{\partial s}
$$

Similar values are obtained in like manner for the other components. If $(l, m, n)$ be the direction-cosines of $P P^{\prime}$, we have

$$
\begin{aligned}
\frac{\partial u}{\partial s} & =\frac{\partial u}{\partial x} \frac{d x}{d s}+\frac{\partial u}{\partial y} \frac{d y}{d s}+\frac{\partial u}{\partial z} \frac{d z}{d s} \\
& =l \frac{\partial u}{\partial x}+m \frac{\partial u}{\partial y}+n \frac{\partial u}{\partial z}, . \\
u & =l q, \quad v=m q, \quad w=n q .
\end{aligned}
$$

whilst

Hence we may write (3) in the form

$$
\frac{\partial u}{\partial t}+u \frac{\partial u}{\partial x}+v \frac{\partial u}{\partial y}+w \frac{\partial u}{\partial z}
$$

which is familiar to students of Hydrodynamics.

It has been thought worth while, as a matter of principle, to accentuate the changed point of view, but in the application to motions which are treated as infinitely slow the distinction loses its importance. The second term in (3) is then of the second order in the velocities, and the component particleaccelerations may be identified with $\partial u / \partial t, \partial v / \partial t, \partial w / \partial t$. The extent of the error here involved, in acoustical questions, may be estimated as in $\S 60$ by a reference to plane waves of sound. If

$$
\xi=a \cos k(c t-x),
$$


the ratio of the maximum value of $u \partial u / \partial x$ to $\partial u / \partial t$ is $k a$. The restriction to "infinitely slow" motions therefore means that the amplitude must be small compared with $\lambda / 2 \pi$.

If we fix our attention on any geometrical surface, open or closed, drawn in the region occupied by the fluid, the expression

$$
(l u+m v+n w) \delta S . \delta t,
$$

where $(l, m, n)$ is the direction of the normal drawn from an elementary area $\delta S$ of the surface, towards one side, measures the volume which in the infinitely short time $\delta t$ crosses $\delta S$. The coefficient of $\delta t$ in this expression is called the "flux" across $\delta S$, and its integral

$$
\iint(l u+m v+n w) d S
$$

taken over the surface, is called the total flux across the latter towards the side on which the normals are supposed drawn. It measures the rate at which fluid is being carried across the surface, expressed in terms of volume per unit time.

To calculate the flux outwards across the boundary of an elementary rectangular region $\delta x \delta y \delta z$ having its centre $P$ at the point $(x, y, z)$, we note that the average velocities parallel to $x$, over the faces $\delta y \delta z$, being equal to the values of $u$ at the centres of these faces, will be

$$
u \pm \frac{1}{2} \delta x \cdot \frac{\partial u}{\partial x}
$$

respectively. The difference of the fluxes, from left to right, across these faces is accordingly $\partial u / \partial x . \delta x \delta y \delta z$. Adding the corresponding terms for the other pairs of faces, we obtain the result

$$
\left(\frac{\partial u}{\partial x}+\frac{\partial v}{\partial y}+\frac{\partial w}{\partial z}\right) \delta x \delta y \delta z
$$

The expression in brackets gives a sort of measure of the rate at which the substance in the neighbourhood of $P$ is on the whole flowing away from $P$. It is therefore called the "divergence" "of the vector $(u, v, w)$, and is denoted by $\operatorname{div}(u, v, w)$; thus

$$
\operatorname{div}(u, v, w)=\frac{\partial u}{\partial x}+\frac{\partial v}{\partial y}+\frac{\partial w}{\partial z}
$$


By dividing any finite region into rectangular elements we see that the total flux outwards across the boundary must be equal to the volume-integral of the divergence, or

$$
\iiint\left(\frac{\partial u}{\partial x}+\frac{\partial v}{\partial y}+\frac{\partial w}{\partial z}\right) d x d y d z=\iint(l u+m v+n w) d S
$$

This can of course be proved mathematically without attributing any kinematical meaning to the symbols.

\section{Equations of Motion.}

To form the dynamical equations, we fix our attention on that portion of matter which at the instant $t$ occupies the rectangular space $\delta x \delta y \delta z$. On the hypothesis of infinitely slow motion its acceleration of momentum parallel to $x$ is $\rho \delta x \delta y \delta z . \partial u / \partial t$, where $\rho$ is the density. The mean pressures on the respective faces may be taken to be the pressures at the centres of those faces, and the total pressures on the two faces perpendicular to $x$ are therefore

$$
\left(p \pm \frac{\partial p}{\partial x} \cdot \frac{1}{2} \delta x\right) \delta y \delta z
$$

The difference gives a force $-\partial p / \partial x . \delta x \delta y \delta z$ in the direction of $x$-positive. Equating this to the acceleration of momentum, we obtain the first of the following system of equations :

$$
\rho \frac{\partial u}{\partial t}=-\frac{\partial p}{\partial x}, \quad \rho \frac{\partial v}{\partial t}=-\frac{\partial p}{\partial y}, \quad \rho \frac{\partial w}{\partial t}=-\frac{\partial p}{\partial z} .
$$

Since the variations of $\rho$ when multiplied by $\partial u / \partial t, \ldots, \ldots$ may be neglected, we may replace $\rho$ by its equilibrium value $\rho_{0}$, but it will not always be necessary to preserve the suffix.

As in $\S 59$ we write

$$
p=p_{0}+\kappa s,
$$

where $s$ denotes the condensation $\left(\rho-\rho_{0}\right) / \rho_{0}$, and $\kappa$ is the cubic elasticity of the fluid. If we further write

as before, we obtain

$$
c^{2}=\kappa / \rho_{0},
$$

$$
\frac{\partial u}{\partial t}=-c^{2} \frac{\partial s}{\partial x}, \quad \frac{\partial v}{\partial t}=-c^{2} \frac{\partial s}{\partial y}, \quad \frac{\partial w}{\partial t}=-c^{2} \frac{\partial s}{\partial z}
$$


If $\Delta$ denote the dilatation of volume of the fluid which at the instant $t$ fills the space $\delta x \delta y \delta z$, as compared with its equilibrium condition, we evidently have

$$
\frac{\partial \Delta}{\partial t}=\operatorname{div}(u, v, w)
$$

or since, in the case of small motions, $s=-\Delta$,

$$
\frac{\partial s}{\partial t}=-\left(\frac{\partial u}{\partial x}+\frac{\partial v}{\partial y}+\frac{\partial w}{\partial z}\right)
$$

The equations (4), (6) are fundamental in the present branch of our subject. The purely kinematical relation (6) is sometimes called the "equation of continuity."

\section{Velocity-Potential.}

If we integrate the equations (4) of $\S 68$ with respect to $t$ we obtain

$$
\left.\begin{array}{c}
u=-c^{2} \frac{\partial}{\partial x} \int_{0}^{t} s d t+u_{0}, \quad v=-c^{2} \frac{\partial}{\partial y} \int_{0}^{t} s d t+v_{0}, \\
w=-c^{2} \frac{\partial}{\partial z} \int_{0}^{t} s d t+w_{0},
\end{array}\right\}
$$

where $u_{0}, v_{0}, w_{0}$ are the values of $u, v, w$ at the point $(x, y, z)$ at the instant $t=0$. In a large class of cases, these initial values of $u, v, w$ can be expressed as the partial differential coefficients of a single-valued function of $(x, y, z)$, thus

$$
u_{0}=-\frac{\partial \phi_{0}}{\partial x}, \quad v_{0}=-\frac{\partial \phi_{0}}{\partial y}, \quad w_{0}=-\frac{\partial \phi_{0}}{\partial z}
$$

Throughout any region to which this statement applies, the values of $u, v, w$ at any subsequent instant $t$ can be similarly expressed ; thus, from (1),

$$
u=-\frac{\partial \phi}{\partial x}, \quad v=-\frac{\partial \phi}{\partial y}, \quad w=-\frac{\partial \phi}{\partial z},
$$

where

$$
\phi=c^{2} \int_{0}^{t} s d t+\phi_{0}
$$

This function $\phi$ is called a "velocity-potential," owing to its analogy with the potential-function which occurs in the theories of Attractions, Electrostatics, \&c. It was introduced into hydrodynamics by Lagrange. 
The instantaneous configuration of the "equipotential surfaces" $\phi=$ const. indicates at once the distribution of velocity, as regards both magnitude and direction. Suppose two consecutive surfaces to be drawn, for which the values of $\phi$ differ by $\delta \phi$. Let $P P^{\prime}$ be drawn normal to these, and $P P_{1}$ parallel to $x$; and let $P P^{\prime}=\delta \nu$. According to (3) the velocity at $P$, resolved

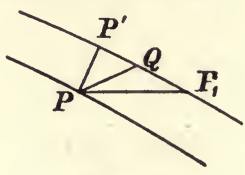

Fig. 64 . in the direction $P P_{1}$, is

$$
u=-\frac{\delta \phi}{P P_{1}}=-\frac{\delta \phi}{\delta \nu} \cdot \frac{P P^{\prime}}{P P_{1}}=-l \frac{\partial \phi}{\partial \nu},
$$

ultimately, if $l$ denote the cosine of the angle which the normal $P P^{\prime}$ makes with $O x$. From this, and from the analogous forms of $v, w$, it is seen that the velocity at $P$ is normal to the equipotential surface passing through that point, and is equal in magnitude to the limiting value of $\delta \phi / \delta \nu$. Hence if a system of surfaces be drawn corresponding to values of $\phi$ which differ by equal infinitesimal amounts, the velocity is everywhere orthogonal to these, and inversely proportional to $\delta \nu$, the distance between consecutive surfaces. More precisely, the velocity is everywhere in the direction in which $\phi$ decreases* most rapidly, and is equal in absolute value to the gradient of $\phi$.

If we draw a linear element $P Q(=\delta s)$ in any other direction, the velocity resolved in the direction of $P Q$ is equal to the limit of

or $-\partial \phi / \partial s$.

$$
-\frac{\delta \phi}{\delta \nu} \cdot \cos P^{\prime} P Q,=-\frac{\delta \phi}{\delta s},
$$

The cases in which a velocity-potential exists include all those where, in the region considered, the fluid was initially at rest, for we may then put $\phi_{0}=0$, simply, and the subsequent value is

$$
\phi=c^{2} \int_{0}^{t} s d t
$$

This will hold whenever the motion has been originated by the vibration of solid or other bodies.

* It should be mentioned that in many books $\phi$ is taken with the opposite sign; thus $u=\partial \phi / \partial x, \& \mathrm{c}$. 
The real meaning of the property which differentiates the present type of motion from all others is most clearly expressed in terms of the "circulation" round a closed curve. If we divide the curve into infinitesimal linear elements, and multiply the length of each element by the tangential component of the velocity, estimated always in the same direction round the curve, the result is the "circulation" referred to. It may be denoted by

$$
\int\left(u \frac{d x}{d s}+v \frac{d y}{d s}+w \frac{d z}{d s}\right) d s, \text { or } \int(u d x+v d y+w d z) .
$$

On the present hypothesis the tangential velocity is $-\partial \phi / \partial s$, and the integral of this, taken round the circuit, is zero, the first and last values of $\phi$ being the same. The circulation is therefore zero in every circuit which can be drawn in the region in question. For a reason which may be understood by reference to the case of an infinitesimal circuit, the type of motion now under consideration is called "irrotational." The name has the advantage of calling attention to a geometrical property rather than to an analytical form of expression.

A dynamical interpretation can also be given to the velocity-potential. The equations (3), when written in the forms

$$
\rho_{0} u=-\rho_{0} \partial \phi / \partial x, \quad \rho_{0} v=-\rho_{0} \partial \phi / \partial y, \quad \rho_{0} w=-\rho_{0} \partial \phi / \partial z,
$$

shew that $\phi$ is the potential per unit mass of a system of extraneous impulsive forces which would generate the actual motion of the fluid instantaneously from rest.

The theorem as to the persistence of the irrotational character is most important; but it is necessary to observe the restrictions under which it has been proved. It was implied, in the first place, that the fluid was frictionless, and this is essential. Again the medium has been supposed free from extraneous forces, but the restriction is easily removed in the case of forces which, like gravity, have a potential (per unit mass). Finally, the assumption has been made that the motion is infinitely small. This simplifies the proof, and covers most cases which are of interest in acoustics. A more rigorous investigation would shew that the circulation is (under the above condition) still 
constant round any circuit, provided we imagine the circuit to move with the fluid. If initially zero for every circuit which can be drawn in a finite portion of the fluid, it will remain zero for every such circuit.

\section{General Equation of Sound Waves.}

We postulate henceforth the existence of a velocity potential, at all events in the case of a uniform medium, to which we confine ourselves for the present. We have then, from $\S 68(6)$

$$
\frac{\partial s}{\partial t}=\nabla^{2} \phi
$$

where

$$
\nabla^{2}=\frac{\partial^{2}}{\partial x^{2}}+\frac{\partial^{2}}{\partial y^{2}}+\frac{\partial^{2}}{\partial z^{2}}
$$

This symbol $\nabla^{2}$ is called the "Laplacian operator," from its constant occurrence in the analytical theory of attractions as first developed by Laplace. Again, by differentiation of $§ 69$ (4) with respect to $t$ we get

$$
c^{2} s=\frac{\partial \phi}{\partial t} .
$$

Finally, by elimination of $s$,

$$
\frac{\partial^{2} \phi}{\partial t^{2}}=c^{2} \nabla^{2} \phi
$$

This may be regarded as the general differential equation of sound waves in a uniform medium. If a solution can be obtained which gives prescribed initial values to $\phi$ and $s$ (or $\partial \phi / \partial t$ ), and satisfies the other conditions of the problem, the subsequent value of $s$ is given by (3), and the values of $u, v, w$ by $§ 69(3)$.

We may stop for a moment to notice the form assumed by the equations when the fluid is incompressible. This may be regarded as an extreme case, in which $c$ is made infinite, whilst $s$ is correspondingly diminished, in such a way that $c^{2} s$, which $=\left(p-p_{0}\right) / \rho_{0}$, remains finite. The equation of continuity, $\S 68$ (6), takes the form

$$
\frac{\partial u}{\partial x}+\frac{\partial v}{\partial y}+\frac{\partial w}{\partial z}=0
$$


which is otherwise obvious from the meaning of "divergence." In the case of irrotational motion, this becomes

$$
\nabla^{2} \phi=0,
$$

which is identical with "Laplace's equation" in the theory of attractions. The same equation occurs in the theory of steady electric (or thermal) conduction in metals. If, for example, $\phi$ denote the electric potential, the formulae (3) of $§ 69$ give the components of current, provided the specific resistance of the substance be taken to be unity. This analogy will be found useful in the sequel.

The theory of the motion of incompressible fluids is capable of throwing more light, occasionally, on acoustical phenomena than might at first sight be anticipated. We are apt to forget that the velocity with which changes of pressure are propagated in water is after all only four or five times as great as in air, and that the visible (or at all events easily imaginable) motions of water, under circumstances where the compressibility has obviously little influence, may supply a valuable hint as to the behaviour of a gaseous substance under similar conditions. This remark will have frequent illustration in the following chapters.

The kinetic energy of a system of sound waves is

$$
\begin{aligned}
T & =\frac{1}{2} \rho \iiint\left(u^{2}+v^{2}+w^{2}\right) d x d y d z \\
& =\frac{1}{2} \rho \iiint\left\{\left(\frac{\partial \phi}{\partial x}\right)^{2}+\left(\frac{\partial \phi}{\partial y}\right)^{2}+\left(\frac{\partial \phi}{\partial z}\right)^{2}\right\} d x d y d z .
\end{aligned}
$$

The potential energy, as given by the argument of $\S 60$, is

$$
V=\frac{1}{2} \kappa \iiint s^{2} d x d y d z=\frac{1}{2} \frac{\rho}{c^{2}} \iiint\left(\frac{\partial \phi}{\partial t}\right)^{2} d x d y d z .
$$

The integrations extend over the region affected.

\section{Spherical Waves.}

In the case of plane waves with fronts perpendicular to $O x$ the equation (4) of $\S 70$ reduces to

$$
\frac{\partial^{2} \phi}{\partial t^{2}}=c^{2} \frac{\partial^{2} \phi}{\partial x^{2}},
$$

whence

$$
\phi=f(c t-x)+F(c t+x) \text {. }
$$

This need not be further discussed. 
The case which comes next in importance is that of symmetrical spherical waves. If $\phi$ be a function of the distance $r$ from the origin and of $t$, only, the velocity is $-\partial \phi / \partial r$ outwards, in the direction of the radius, and is uniform over any spherical surface having the origin $O$ as centre.

Instead of applying the general equation to the present circumstances it is simpler to form the kinematical relation corresponding to $\$ 70$ (1) de novo. The flux outwards across a sphere of radius $r$ is $-\partial \phi / \partial r .4 \pi r^{2}$, and the difference of flux across the outer and inner surfaces of a spherical shell of thickness $\delta r$ is accordingly

$$
-4 \pi \frac{\partial}{\partial r}\left(r^{2} \frac{\partial \phi}{\partial r}\right) \delta r
$$

The volume of the shell being $4 \pi r^{2} \delta r$, this must be equal to $\dot{\Delta} .4 \pi r^{2} \delta r$ or $-\dot{s} .4 \pi r^{2} \delta r$, whence

$$
r^{2} \frac{\partial s}{\partial t}=\frac{\partial}{\partial r}\left(r^{2} \frac{\partial \phi}{\partial r}\right)
$$

Since

$$
c^{2} s=\frac{\partial \phi}{\partial t}
$$

as usual, we have $\quad \frac{\partial^{2} \phi}{\partial t^{2}}=\frac{c^{2}}{r^{2}} \frac{\partial}{\partial r}\left(r^{2} \frac{\partial \phi}{\partial r}\right)$.

This may also be written

$$
\frac{\partial^{2}(r \phi)}{\partial t^{2}}=c^{2} \frac{\partial^{2}(r \phi)}{\partial r^{2}}
$$

The solution of this equation, viz.

$$
r \phi=f(c t-r)+F(c t+r),
$$

represents the superposition of two wave-systems travelling outwards and inwards, respectively, with the velocity $c$. In the case of a diverging wave-system

$$
r \phi=f(c t-r)
$$

we have, by (4),

$$
c r s=f^{\prime}(c t-r) \text {. }
$$

Any value of $r s$ is propagated unchanged; the condensation $s$ therefore diminishes in the ratio $1 / v$ as it proceeds, and the potential energy per unit volume diminishes as $1 / r^{2}$. For the particle-velocity we have

$$
q=-\frac{\partial \phi}{\partial r}=\frac{1}{r} f^{\prime}(c t-r)+\frac{1}{r^{2}} f(c t-r) .
$$


The law of dependence on distance is here more complicated, but as the wave spreads outwards the first term ultimately predominates; the velocity at corresponding points of the wave then varies as $1 / r$, and the kinetic energy per unit volume as $1 / r^{2}$.

In a diverging wave-system we have, from (9),

$$
c r s=-\frac{\partial}{\partial r}(r \phi)
$$

and similarly, in a converging wave-system,

$$
c r s=\frac{\partial}{\partial r}(r \phi) .
$$

These relations correspond to (5) of $\S 60$, which is indeed a particular case, since as $r$ increases our spherical waves tend to become ultimately plane.

The general argument of $\S 23$ can be adduced to prove that in a diverging (or a converging) wave-system by itself the energy is half kinetic and half potential.

The solution (7) can be applied to a region included between concentric spheres, or to a region having only one finite spherical boundary, internal or external. In any case, the conditions to be satisfied at the boundaries, whether finite or infinite, must be given in order that the problem may be determinate. In particular, even when the region is otherwise unlimited, the point $r=0$ is to be reckoned as an internal boundary; this point might for instance be occupied by a "source" of sound ( $§ 73)$. When there is no source there, the flux across a small spherical surface surrounding $O$ must vanish, i.e. we must have

$$
\lim _{r=0}\left(-\frac{\partial \phi}{\partial r} \cdot 4 \pi r^{2}\right)=0 .
$$

When applied to (7) this condition gives

$$
f(c t)+F(c t)=0
$$

for all values of $t$, and the general solution therefore takes the shape

$$
r \phi=F(c t+r)-F(c t-r) .
$$

This formula may be used to determine the motion consequent on arbitrary initial conditions which are symmetrical 
about 0 , in an unlimited medium. Suppose that when $t=0$ we have

$$
\phi=\phi_{0}(r), \quad \frac{\partial \phi}{\partial t}=\chi_{0}(r)
$$

The former of these functions determines the initial distribution of velocity, and the latter that of condensation. The function $F$ must now satisfy the conditions

$$
\begin{aligned}
& F(r)-F(-r)=r \phi_{0}(r), \\
& F^{\prime}(r)-F^{\prime}(-r)=\frac{r}{c} \chi_{0}(r) .
\end{aligned}
$$

It is to be noted that the variable $r$ is essentially positive; this explains why two equations are necessary to determine $F$ for positive and negative values of the argument.

Suppose, for example, that there is no initial velocity anywhere, but only an initial condensation, so that $\phi_{0}(r)=0$. From (17) and (18) we deduce

$$
F^{\prime}(r)=-F^{\prime}(-r)=\frac{1}{2} \frac{r}{c} \chi_{0}(r)
$$

The condensation at time $t$ is given by

$$
s=\frac{1}{c^{2}} \frac{\partial \phi}{\partial t}=\frac{F^{\prime \prime}(c t+r)-F^{\prime \prime}(c t-r)}{c r} .
$$

This takes different forms according as $c t$ is less or greater than $r$. In the former case

$$
s=\frac{1}{2 c^{2} r}\left\{(r+c t) \chi_{0}(r+c t)+(r-c t) \chi_{0}(r-c t)\right\},
$$

and in the latter

$$
s=\frac{1}{2 c^{2} r}\left\{(c t+r) \chi_{0}(c t+r)-(c t-r) \chi_{0}(c t-r)\right\} .
$$

As a particular case, suppose we have an initial condensation which is uniform $\left(=s_{0}\right)$ throughout the interior of a sphere of radius $a$, and vanishes for $r>a$; and let us examine the subsequent variations of $s$ at points outside the originally disturbed region. Since $\chi_{0}(r)$ vanishes by hypothesis for $r>a$, the first part of the solution (21) or (22) disappears in the 
present case. So long as $c t<r-a$, the second part of (21) will also vanish, but when $c t$ lies between $r-a$ and $r$ we shall have

$$
s=\frac{s_{0}}{2 \dot{r}}(r-c t) \text {. }
$$

When $c t>r$, the second formula (22) applies, and we find that, so long as $c t<r+a$, the result (23) will still hold. Finally, when $c t>r+a$ we have again $s=0$. The results are shewn graphically in the following figure which exhibits the variation of $s$ with $t$ at a particular point, and the space-
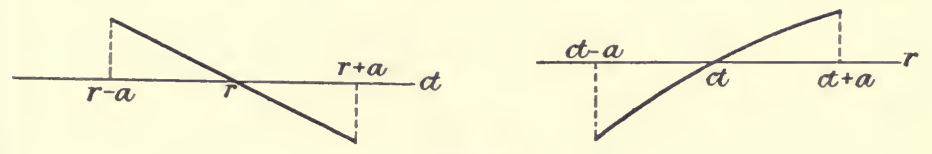

Fig. 65 .

distribution of $s$ at a particular instant, respectively. It appears that after the lapse of a certain time $(2 a / c)$ we have a diverging wave in the form of a spherical shell of thickness $2 a$, and that $s$ is positive through the outer half, and negative through the inner half of the thickness. The changes in the velocity may be inferred by means of the formula $q=-\partial \phi / \partial r$. For values of $t$ between $(r-a) / c$ and $(r+a) / c$, i.e. during the time of transit of the wave across the point considered, we find

$$
\phi=c^{2} \int_{0}^{t} s d t=\frac{c s_{0}}{4 r}\left\{a^{2}-(r-c t)^{2}\right\} ;
$$

whilst for other values of $t$ we have $\phi=0$. Hence within the aforesaid limits of time we have

$$
q=-\frac{\partial \phi}{\partial r}=\frac{c s_{0}}{4 r^{2}}\left(a^{2}+r^{2}-c^{2} t^{2}\right)
$$

When $r$ is large compared with $a$ this changes sign for $t=r / c$, approximately, the velocity being directed outwards in the outer half, and inwards in the inner half of the shell. At the boundaries of the disturbed region, where $r=c t \pm a$, we have $q= \pm c a s_{0} / 2 r$. As the diverging wave reaches any point the velocity suddenly rises from zero to the former of these values, and as it leaves it the velocity falls suddenly from the latter (negative) value to 0 . The origin of the discontinuities in this 
solution is to be sought of course in the discontinuity of the initial distribution of density. Any difficulty which may be felt on such grounds may in general be removed by substituting in imagination an initial distribution in which the discontinuity is replaced by a very rapid but continuous transition.

The solution of (6) in terms of the general initial conditions (16) may be investigated in a similar manner, but it must suffice to quote the results. It may easily be verified that they satisfy all the conditions of the question. They are

$$
\begin{aligned}
r \phi=\frac{1}{2}(r+c t) \phi_{0}(r+c t)+\frac{1}{2}( & r-c t) \phi_{0}(r-c t) \\
& +\frac{1}{2 c} \int_{r-c t}^{r+c t} z \chi_{0}(z) d z
\end{aligned}
$$

for $c t<r$, and

$$
\begin{aligned}
& r \phi=\frac{1}{2}(c t+r) \phi_{0}(c t+r)-\frac{1}{2}(c t-r) \phi_{0}(c t-r) \\
& \quad+\frac{1}{2 c} \int_{c t-r}^{c t+r} z \chi_{0}(z) d z \ldots(
\end{aligned}
$$

for $c t>r$.

Since the origin evidently occupies an exceptional position in the theory of spherical waves it is desirable to calculate the value of $\phi$ there, more especially as the result will be of service presently when we come to the solution of the general equation $\S 70$ (4) of sound waves. The result may be deduced from (27), or more directly from (15). We find

$$
\phi=\lim _{r=0} \frac{F(c t+r)-F(c t-r)}{r}=2 F^{\prime}(c t),
$$

and therefore from (17) and (18)

$$
\begin{aligned}
\phi & =t \chi_{0}(c t)+\phi_{0}(c t)+c t \phi_{0}{ }^{\prime}(c t) \\
& =t \chi_{0}(c t)+\frac{d}{d t} \cdot t \phi_{0}(c t) . \quad \ldots . .
\end{aligned}
$$

For example, in the special problem above considered, where $\phi_{0}(r)=0$, whilst $\chi_{0}(r)=c^{2} s_{0}$ or 0 according as $r \lessgtr a$, we find $\phi=c^{2} s_{0} t$ or 0 according as $t \leqslant a / c$. The consequent value of $s$ at $O$ is $s_{0}$ for $t<a / c$ and zero for $t>a / c$, whilst at the instant $t=a / c$ it is negative infinite. To escape this result we must slightly modify the data, replacing the original distribution of density by a continuous one. The figure is an attempt to 
shew an initial distribution of $s$ which varies rapidly but continuously from $s_{0}$ to 0 in the neighbourhood of $r=a$, together with the consequent time-variation of $s$ at 0 .
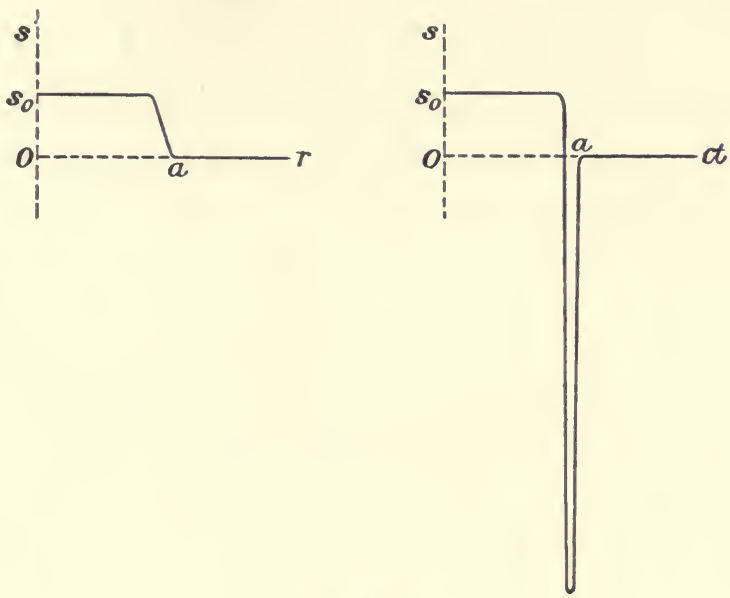

Fig. 66 .

The problem which we have discussed exhibits a marked contrast with the theory of plane waves, in that the wave resulting from an arbitrary disturbance contains both condensed and rarefied portions, even when there is no initial velocity and the initial disturbance of density has everywhere the same sign. The statement is easily generalized by means of equations (1) of $\S 69$. If we take the integral of the value of $s$ at any point $P$ over a time which covers the whole transit of the wave, so that the values of $u, v, w$ vanish at both limits, we find that its space-derivatives are all zero. The integral has therefore the same value for all positions of $P$. And by taking $P$ at an infinite distance, so that $s$ becomes infinitely small by spherical divergence, we see that the value is in fact zero, i.e.

$$
\int s d t=0
$$

The mean value of $s$ at any point is therefore zero. This result is of course not limited to the case of spherical waves. 
72. Waves resulting from a given Initial Disturbance.

We have next to trace the effect of initial conditions in an unlimited region, in the general case. We suppose that at the instant $t=0$ we have

$$
\phi=\phi_{0}(x, y, z), \quad \frac{\partial \phi}{\partial t}=\chi_{0}(x, y, z)
$$

where the functions are arbitrary. To deduce the effect at any subsequent instant, at any assigned point $P$, we consider in the first instance the average value of $\phi$ over a sphere of radius $r$ described with $P$ as centre. This will be denoted by

$$
\bar{\phi}=\frac{1}{4 \pi} \iint \phi d \omega
$$

if $\delta \omega$ represent the elementary solid angle $\left(\delta S / r^{2}\right)$ subtended at $P$ by any elementary area $\delta S$ of the sphere. In the same way we write

$$
\bar{s}=\frac{1}{4 \pi} \iint s d \omega .
$$

This, like (2), will be a function of the variables $r$ and $t$ only. If in $\$ 70$ (3) we multiply both sides by $\delta \omega / 4 \pi$, and integrate over the aforesaid sphere of radius $r$, we find

$$
c^{2} \bar{s}=\frac{\partial \bar{\phi}}{\partial t} .
$$

It is also evident that the average normal velocity over the sphere will be $-\partial \bar{\phi} / \partial r$. The argument by which the rate of change of $s$ was in $\$ 71$ inferred from the consideration of the total flux out of the region bounded by the spheres $r$ and $r+\delta r$ can then be applied to prove that in the present case

$$
\frac{d \vec{s}}{d t}=\frac{1}{r^{2}} \frac{\partial}{\partial r}\left(r^{2} \frac{\partial \bar{\phi}}{\partial r}\right)
$$

Eliminating $\bar{s}$, we have

$$
\frac{\partial^{2} \bar{\phi}}{\partial t^{2}}=\frac{c^{2}}{r^{2}} \frac{\partial}{\partial r}\left(r^{2} \frac{\partial \bar{\phi}}{\partial r}\right)
$$

which is identical in form with (5) of $\S 71$. We recognize then that $\bar{\phi}$ is the velocity-potential of the system of spherical waves 
which would result from initial distributions of velocity and condensation expressed by

$$
\phi=\bar{\phi}_{0}(r), \quad \frac{\partial \phi}{\partial t}=\bar{\chi}_{0}(r)
$$

these functions of $r$ being the average values of $\phi_{0}(x, y, z)$ and $\chi_{0}(x, y, z)$ taken over the aforesaid sphere. It follows from $\S 71$ (29) that the value of $\phi$ at $P$ is given by

$$
\phi_{P}=\frac{d}{d t} \cdot t \bar{\phi}_{0}(c t)+t \bar{\chi}_{0}(c t) \text {. }
$$

This gives a rule for calculating the value of $\phi$ for a point $P$ at any given instant $t$. It may be stated in words as follows:

To find the part of $\phi$ due to the given initial distribution of condensation, we describe about $P$ a sphere of radius $c t$, and calculate the average of the given initial values of $\partial \phi / \partial t$, i.e. of the function $\chi_{0}(x, y, z)$, at the points of space through which this surface passes, and multiply by $t$. To find the part due to the initial velocities we replace the average of the given values of $\partial \phi / \partial t$ by the average of the given initial values of $\phi$, i.e. of the function $\phi_{0}(x, y, z)$, and differentiate the result, as thus modified, with respect to $t$.

The theorem contained in (8) was given by Poisson (1819); the actual form (8) and the interpretation are due to Stokes (1850). It will be seen that the result, as thus stated, is in reality very simple, if regard be had to the great generality of the circumstances which are taken into account.

To trace the sequence of events at $P$ we employ a series of spheres whose radii (ct) increase continually from zero. If $P$ be external to the region which is the locus of the initial disturbance, no effect is produced so long as the spheres do not encroach on this region. If $r_{1}, r_{2}$ be the least and greatest distances of $P$ from the boundary, the disturbance at $P$ will begin after a time $r_{1} / c$, will last for a time $\left(r_{2}-r_{1}\right) / c$, and will then cease.

If with the various points of the boundary of the originally disturbed region as centres we describe a series of spheres of radius $c t$, the outer sheet of the envelope of these spheres will mark out the boundary of the space which has been invaded by 
the disturbance up to the instant $t$. The envelopes corresponding to successive values of $t$ will form a series of what are known in geometry as "parallel surfaces"; in other words, the boundary of the disturbed region spreads everywhere normal to itself with the constant velocity $c$.

As a simple application of the formula (8) we may take the problem already discussed in $\S 71$, where an initial uniform condensation $s_{0}$ was supposed to extend throughout the interior of a sphere of radius $a$ having the origin $O$ as centre. When a spherical surface of radius $c t$, described with $P$ as centre, intersects the boundary of the originally disturbed region, as in the figure, the area of the portion

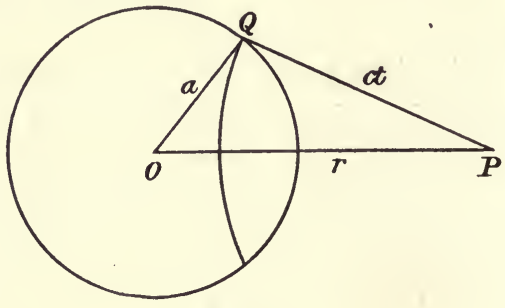

Fig. 67. included within the latter is $2 \pi \cdot P Q^{2}(1-\cos O P Q)$, and the average of the given initial values of $s$ over the whole surface $\left(4 \pi . P Q^{2}\right)$ is therefore

$$
\frac{1}{2}(1-\cos O P Q) s_{0}={\frac{a^{2}-(c t-r)^{2}}{4 c t r}}^{2},
$$

where $r=O P$. Hence, by the rule,

$$
\phi_{P}=\frac{c s_{0}}{4 r}\left\{a^{2}-(c t-r)^{2}\right\},
$$

in agreement with $\S 71$ (24).

\section{Sources of Sound. Reflection.}

The very useful conception of a "point-source" was introduced into the subject by Helmholtz. We may imagine (with Maxwell and Lord Rayleigh) that at such a point fluid is introduced or abstracted at a certain rate, and that the "strength" of the source is measured by the volume thus introduced per unit time. The wave-train due to a source of strength $f(t)$ at the origin is accordingly represented by

$$
4 \pi r \phi=f\left(t-\frac{r}{c}\right) \text {, }
$$


since this makes

$$
\lim _{r=0}\left(-\frac{\partial \phi}{\partial r} \cdot 4 \pi r^{2}\right)=f(t) .
$$

If we differentiate the general equation of sound waves ((4) of $\S 70$ ) with respect to $x$ or $y$ or $z$, we recognize that if $\phi$ is a solution so also is $\partial \phi / \partial x$, or $\partial \phi / \partial y$, or $\partial \phi / \partial z$. Thus from (1) we derive the solution

$$
4 \pi \phi=\frac{\partial}{\partial x} \cdot \frac{f(t-r / c)}{r},
$$

which satisfies the general differential equation except at the singular point $r=0$. The value of $\phi$ thus obtained may be interpreted as the velocity-potential of a "double source" due to the juxtaposition of two simple sources which are always in opposite phases. This will be explained more fully in $\S 76$, in the particular case where the variation with time is simpleharmonic.

The problem of reflection of sound by a rigid infinite plane is readily solved by the method of "images." If with every source $P$ of sound on the near side of the boundary we associate a similar source at the geometrical image $P^{\prime}$ of $P$ with respect to the plane, it is obvious that the condition of zero normal velocity over the plane would still be fulfilled if the boundary were abolished. Hence, in the actual case, the motion on the near side will be made up of that due to the given sources $P$ and of that due to the images $P^{\prime}$. It may be mentioned that the present case of a rigid plane boundary is the only one where the physical "image" of a point-source is itself accurately a point-source.

The problem of reflection at the plane boundary of two distinct fluid media has been discussed in $\S 61$, in the case of direct incidence. The case of oblique reflection was solved by Green (1847). The results are chiefly of interest for the sake of the optical analogies, but one curious point, noticed by Helmholtz, may be mentioned. Owing to the greater velocity of sound in water, the conditions for total reflection may occur when the waves are incident from air on water (in fact whenever the angle of incidence exceeds about $13^{\circ}$ ), but not in the converse case. This is of course the reverse of what holds with regard to light. 


\section{Refraction due to Variation of Temperature.}

Questions relating to wave-propagation in heterogeneous media can only be discussed in a general way, and with the help of conceptions borrowed from geometrical optics. If at any surface there is an abrupt change of properties the law of propagation is of course altered. If the dimensions of the surface, and its radii of curvature, are large compared with the wave-length, we have phenomena of regular reflection and refraction, as in optics. Cases of absolute discontinuity are of course not met with in the atmosphere, but the theory would be practically unaffected if the change of properties were effected within a space which is small compared with the wave-length.

When on the other hand we have a continuous variation such that the change of properties within a wave-length is negligible, the case is analogous to that of atmospheric refraction of light, which is discussed in books on optics and astronomy. In an atmosphere of the same gas, at rest, a variation in the velocity of sound can only arise through a variation of temperature $(\$ 59)$. The refraction due to variation of temperature with altitude was first discussed by Osborne Reynolds (1876). Suppose that, as usually happens, the temperature diminishes upwards. Since the velocity of sound varies as the square root of the absolute temperature, the lower portions of a wave-front will be propagated faster than the upper ones, so that a front which was originally vertical gets tilted upwards more and more as it proceeds. The sound will therefore, for the most part, pass over the head of an observer at a sufficient distance, such residual effects as he perceives being referable to diffraction. On the other hand, whenever the temperature increases upwards the waves will be tilted downwards, and the effect at a distance will be greater than if the temperature had been uniform. This latter condition of the atmosphere sometimes prevails on a clear night following a warm day, when, owing to the cooling of the ground by radiation, the lower strata of the atmosphere are reduced in temperature relatively to the upper ones.

The theory has been further developed by Lord Rayleigh, by means of the conception of rays of sound. The surfaces of 
equal wave-velocity being supposed to be horizontal, each ray will travel in a vertical plane. The curvature of a ray may be calculated directly by a method due to Prof. James Thomson*. If $R$ be the radius of curvature, the two wave-fronts passing through the extremities of an element $\delta s$ of the path will be inclined at an angle $\delta s / R$, and if $\delta s^{\prime}$ be the length intercepted on an adjacent ray in the same vertical plane, we have

$$
\delta s^{\prime}=\left(1-\frac{\delta n}{R}\right) \delta s,
$$

where $\delta n$ denotes the distance between the two rays, the standard case being

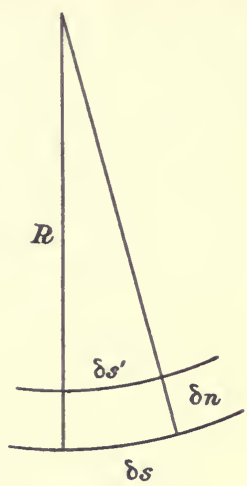

Fig. 68. that shewn in the figure. Since the elements $\delta s, \delta s^{\prime}$ are described in the same time we have

$$
\frac{\delta s^{\prime}}{c+\delta c}=\frac{\delta s}{c}
$$

whence, by comparison with (1),

$$
\frac{1}{R}=-\frac{1}{c} \frac{\partial c}{\partial n}
$$

When the temperature diminishes upwards, $\partial c / \partial n$ is negative and the curvature $1 / R$ is positive, as in the figure, and the rays are curved upwards. But if the temperature increase upwards, the curvature is downwards, so that an observer at the level of the source may hear sounds which would otherwise have been intercepted by obstacles.

The formula (3) leads to the ordinary law of refraction. If $\psi$ be the inclination of the ray to the horizontal we may write

$$
\frac{\partial c}{\partial n}=\frac{d c}{d y} \cos \psi, \quad \frac{\partial c}{\partial s}=\frac{d c}{d y} \sin \psi,
$$

if $y$ be the vertical coordinate. Hence, along the course of a ray,

$$
\frac{d \psi}{d s}=\frac{1}{R}=-\frac{1}{c} \frac{d c}{d s} \cot \psi
$$

* James Thomson (1822 - 92), professor of engineering at Belfast 1857-72, and at Glasgow 1872-89. 
or

$$
c \sec \psi=\text { const., }
$$

which is the law in question. Conversely, from (6) we can derive the formula (3). When $c$ is known as a function of $y$ the equation (6) determines the paths.

The simplest hypothesis is that the temperature decreases (or increases) upwards with a uniform gradient. This includes the particular case of an atmosphere in "convective equilibrium " under gravity, where the gradient is

$$
-\frac{\gamma-1}{\gamma} \cdot \frac{\theta}{H}
$$

$H$ being the height of the homogeneous atmosphere $(§ 59)$ corresponding to the temperature $\theta^{*}$. This is at the rate of about $1^{\circ} \mathrm{C}$. per 100 metres. If a law of uniform decrease were to hold without limitation, we should at a certain altitude meet with a zero temperature (absolute). If for a moment we take the origin at this level, and draw the axis of $y$ downwards, the temperature will be proportional to $y$, and the wave-velocity $c$ to $y^{\frac{1}{2}}$. Hence by (6) we have, along any ray,

$$
y=C \cos ^{2} \psi \text {. }
$$

The paths are therefore cycloids, the generating circles of which roll on the under side of the line $y=0$. If on the other hand the temperature increases upwards with a uniform gradient, the paths are the cycloids whose generating circles roll on the upper side of the line which corresponds to the zero of temperature. In any practical case we are concerned only with the portions of the curves near the vertices. The ares may therefore be taken to be circular, with a radius double the distance below (or above) the level of zero temperature. In the extreme case of upward diminution to which the formula (7) refers, this radius will therefore be (roughly) $2 \times 273 \times 100=54600$ metres, for a temperature of $0^{\circ} \mathrm{C}$.

* It was pointed out by Lord Kelvin (1862) that this is the condition into which the atmosphere would be brought by the free play of convection currents alone, without conduction or radiation. It is therefore one of neutral equilibrium. If the temperature diminish upwards at a greater rate the equilibrium becomes unstable. 


\section{Refraction by Wind.}

Another interesting question is that of refraction by wind. A uniform motion of the medium introduces of course no complication, the relative motion of the sound waves being exactly the same as if the medium were at rest. Usually, however, the wind-velocity near the ground is less than above, the motion of the lower layers of air being obstructed. Hence when a wave-front travels with the wind, the upper portions are propagated (in space) somewhat faster than the lower, the velocity of the wind being superposed on that of sound. The front is therefore continually being tilted downwards. For a similar reason a wave-front travelling against the wind gets tilted upwards, so that the sound tends to pass over the head of an observer at a distance. This explanation of the familiar fact that sound can be heard better, and further from the source, when this lies to windward than when it is to leeward of the observer, was first given by Stokes (1857). The only previous suggestion had been that a sound which has travelled a certain distance with the wind has really traversed a shorter length of air, and has consequently become less attenuated by spherical divergence, than if the wind had been absent. Owing to the smallness : of wind-velocities in comparison with that of sound, this cause is quite inadequate to explain the very marked effects which are observed. The true theory was discovered independently by Reynolds (1874), and confirmed by a number of interesting experiments.

If we proceed to apply optical methods to the question, it is necessary to distinguish, as in the theory of aberration, between the direction of a ray and that of a wave-normal. Let $S_{1}$ represent the position of a wave-front at time $t, S^{\prime}$ the position at time $t+\delta t$ of those particles which were on $S_{1}$, and $S_{2}$ the new position of the wave-

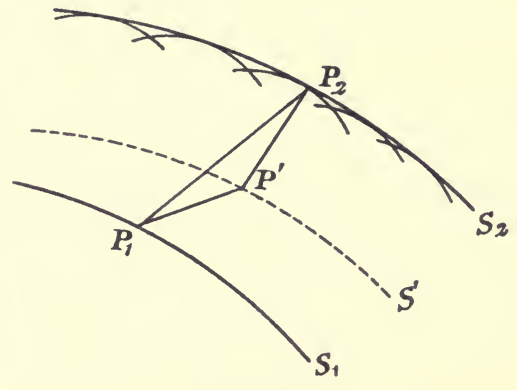

Fig. 69 . 
front. Let $P_{1}$ be any point on $S_{1}$, and $P^{\prime}$ the corresponding point on $S^{\prime}$, so that $P_{1} P^{\prime}$ is the path of a particle of the medium in the time $\delta t$. On the principles of optics, the new position $S_{2}$ of the wave-front is obtained as the envelope of a system of spheres of radius $c \delta t$, described with the various points $P^{\prime}$ of $S^{\prime}$ as centres. If $P_{2}$ be that point on the envelope which corresponds to $P^{\prime}, P_{1} P_{2}$ will be an element of a ray, and $P^{\prime} P_{2}$ an element of the wave-normal. Also since $P_{1} P^{\prime}=U \delta t$, where $U$ is the velocity of the medium, the " rayvelocity" $\left(P_{1} P_{2} / \delta t\right)$ is the resultant of the wave-velocity and the velocity of the medium.

In the present question the velocity $U$ is horizontal, and a function of the altitude $(y)$ only. If $\psi, \phi$ denote the inclinations to the horizontal of the ray and the wave-normal, respectively, we have

$$
\begin{gathered}
\sin (\phi-\psi)=\frac{U}{c} \sin \psi, \\
\phi=\psi+\frac{U}{c} \sin \psi,
\end{gathered}
$$

if $U / c$ be small, as will usually be the case.

To ascertain the law governing the change of direction of the ray, consider first the case of refraction at the common horizontal boundary of two uniform currents $U, U^{\prime}$. If $\phi, \phi^{\prime}$ be the inclinations of the wave-normal on the two sides of the plane of discontinuity, we have

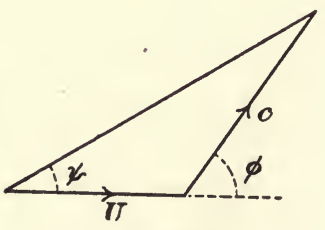

Fig. 70.

$$
c \sec \phi+U=c \sec \phi^{\prime}+U^{\prime},
$$

each side expressing the horizontal velocity of the trace of the wave-front on the plane in question. Since a continuous variation of $U$ can be approximated to by a series of small discontinuities, we infer that (3) will still hold if $\phi, U$ and $\phi^{\prime}, U^{\prime}$ refer to any two positions on the same ray. This gives the altered law of refraction. Lord Rayleigh points out that since $\sec \phi^{\prime} \nless 1, \phi^{\prime}$ will become imaginary if

$$
\left(U^{\prime}-U\right) / c>\sec \phi-1 \text {. }
$$


There is therefore total reflection, at the stratum to which the accents refer, of all wave-fronts whose initial inclination $(\phi)$ to the vertical falls short of a certain limit.

Along any one ray we have

$$
\sec \phi+\frac{U}{c}=\text { const. }
$$

or, by (2), $\quad \sec \psi+\frac{U}{c} \sec ^{2} \psi=$ const.,

provided $\psi$ be not too great. If we differentiate this with respect to the $\operatorname{arc} s$, and put $d \psi / d s=1 / R, d y / d s=\sin \psi$, we find

$$
\frac{1}{R}\left(1+\frac{2 U}{c} \sec \psi\right)=-\frac{1}{c} \frac{d U}{d y}
$$

The ray is therefore curved downwards or upwards, according as $d U / d y$ is positive or negative, i.e. according as the ray is travelling with or against the wind. If the gradient $d U / d y$ be uniform, the rays have all the same uniform curvature, approximately, owing to the smallness of $U / c$, unless indeed the inclination $\psi$ becomes considerable. It

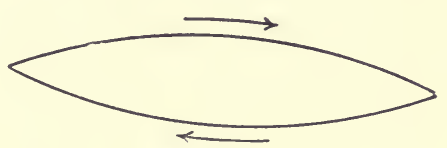

Fig. 71. will be noticed that in this problem the path of a ray is not reversible.

This is a convenient place for a reference to what is known as "Doppler's principle"*. Suppose, for instance, that a periodic source of sound is approaching a stationary observer. The number of maxima of (say) the condensation $s$ which strike the ear of the latter in a second is increased, and the pitch is therefore raised. The diminution in the period is to the period when the source is at rest in the ratio of the velocity of approach to the velocity of sound. When the source recedes from the observer, this ratio is negative, and the pitch is lowered. When the motion of the source is oblique to the rays by which the sound is heard, the com-

* Christian Doppler (1803-54), an Austrian mathematician, professor of physics at Vienna 1851. 
ponent of its velocity in the direction of the ray is alone effective. Analogous effects are produced when the source is at rest and the observer in motion. The principle is exemplified in the apparent change of pitch of the whistle of a locomotive as a train dashes through a station; but its most striking and fruitful applications are met with in the theory of radiation. 


\section{CHAPTER VIII}

\section{SIMPLE-HARMONIC WAVES. DIFFRACTION}

\section{Spherical Waves. Point-Sources of Sound.}

From this point it is convenient to consider specially the case of simple-harmonic vibrations. In problems relating to the impact of sound waves on obstacles, or their transmission by apertures in a screen, and so on, the results will vary in character with the pitch, the determining element being the relation between the wave-length and the linear dimensions of the obstacles, \&c.

It will be desirable, for the sake of conciseness, to use imaginary quantities somewhat more freely than in the preceding chapters. Thus we assume that the velocity-potential $\phi$ varies as $e^{i n t}$, or $e^{i k c t}$, where

$$
k=n / c=2 \pi / \lambda,
$$

if $\lambda$ be the wave-length of plane waves of the same period $2 \pi / n$. The géneral equation of sound waves $(\$ 70(4))$ therefore becomes

$$
\nabla^{2} \phi+k^{2} \phi=0 \text {. }
$$

In the case of plane waves whose fronts are perpendicular to the axis of $x$, we have

$$
\frac{\partial^{2} \phi}{\partial x^{2}}+k^{2} \phi=0
$$

the solution of which may be written

$$
\phi=A e^{-i k x}+B e^{i k x},
$$

$$
\phi=C \cos k x+D \sin k x \text {, }
$$


the time-factor $e^{i n t}$ being understood. Thus a train of simpleharmonic waves travelling in the direction of $x$-positive is represented by

$$
\phi=A e^{i(n t-k x)} .
$$

When we proceed to calculations of energy it is of course necessary to revert to real forms. Thus, taking the real part of (6), we have

$$
\phi=A \cos k(c t-x) \text {. }
$$

The mean energy per unit volume, as given by $\$ 70(7),(8)$, is $\frac{1}{2} \rho k^{2} A^{2}$, and the mean energy transmitted per unit time, per unit area of the wave-front, is

$$
\frac{1}{2} \rho k^{2} c A^{2} \text {, or } \frac{1}{2} \rho n^{2} / c . A^{2} \text {. }
$$

We may call this the "energy-flux" in the wave-system (7).

The equation of symmetrical spherical waves, $\$ 71(6)$, now takes the form

$$
\frac{\partial^{2}(r \phi)}{\partial r^{2}}+k^{2}(r \phi)=0
$$

and the solution is

or

$$
\begin{aligned}
& r \phi=A e^{-i k r}+B e^{i k r}, \\
& r \phi=C \cos k r+D \sin k r,
\end{aligned}
$$

the time-factor being understood as before. The two terms in (10) correspond to waves diverging from, or converging to, the origin, respectively. In particular, the diverging waves due to a source $A e^{i k c t}$ at the origin are represented by

$$
\phi=\frac{A}{4 \pi r} e^{i k(c t-r)}
$$

or, in real form, $\quad \phi=\frac{A}{4 \pi r} \cos n\left(t-\frac{r}{c}\right)$.

This is of course a particular case of $\S 73(1)$.

The maintenance of such a source in an unlimited medium requires a certain expenditure of energy. The work done per unit time at the surface of a sphere of radius $r$, on the fluid outside, is the product of the pressure, the area, and the outward velocity, or

$$
-\left(p_{0}+\kappa s\right) \cdot 4 \pi r^{2} \frac{\partial \phi}{\partial r}=-\left(p_{0}+\rho \frac{\partial \phi}{\partial t}\right) \cdot 4 \pi r^{2} \frac{\partial \phi}{\partial r} .
$$


It is evident that $p_{0}$ contributes nothing to the average effect, since the mean value of $\partial \phi / \partial r$ at any point is zero. If we substitute from (13) we find that the average of the remaining part is

$$
W=\frac{n^{2} \rho A^{2}}{8 \pi c}=\frac{k^{2} \rho c A^{2}}{8 \pi} .
$$

This quantity $W$ is independent of $r$, as was to be anticipated, since the mean energy in the space included between two concentric spheres is constant. It measures the emission of energy (per unit time) by the source. The formula may also be inferred from the consideration that at a great distance the waves may be regarded as plane. If in (8) we replace $A$ by the $A / 4 \pi r$ of (13), and multiply by $4 \pi r^{2}$, we obtain the result (15).

It must be remembered that this calculation of the energy emitted applies only to an isolated source in free space. A source placed in an enclosure with rigid walls does no work on the whole, since the energy of the gas is constant. Even in an open space the emission of energy may be greatly modified by the neighbourhood of an obstacle. Thus in the case of a source $P$ close to a rigid plane boundary the amplitude of vibration at any point is doubled by the reflection as from the image $P^{\prime}$ ( $\$ 73)$; the intensity is quadrupled, and the emission (on one side) is therefore twice that of an equal source in free space.

The equation

$$
\phi=\frac{B}{4 \pi r} e^{i k(c t+r)},
$$

or, in real form,

$$
\phi=\frac{B}{4 \pi r} \cos n\left(t+\frac{r}{c}\right)
$$

may likewise be interpreted as representing a "sink" of sound, i e. a point where energy is absorbed, under similar conditions, at the rate $n^{2} \rho B^{2} / 8 \pi c$. This conception is however of no great assistance in acoustics.

The notion of a simple source, valuable as it is for theoretical purposes, is seldom realized even approximately in practice. A vibrating body such as a membrane, or either prong of a tuning fork, is tending at any instant to produce a condensation of the air in contact with it on the one side and a rarefaction 
on the other, and is therefore more adequately represented, in the simplest cases, by a combination of two simple sources near together but in opposite phases. Idealizing this a little further we are led to the mathematical conception of a "double source." We begin with a simple source of strength $-m$ at a point $O$, and a simple source of strength $+m$ at an adjacent point $O^{\prime}$, the signs indicating the opposition of phase. If we next imagine $m$ to become infinitely great, whilst the distance $0 O^{\prime}$ becomes infinitely small, in such a way that the product $m .00^{\prime}$ remains finite, we have the ideal "double source" of theory. The direction $0 O^{\prime}$ is called the " axis," and the limit of $\mathrm{m} .0 \mathrm{O}^{\prime}$

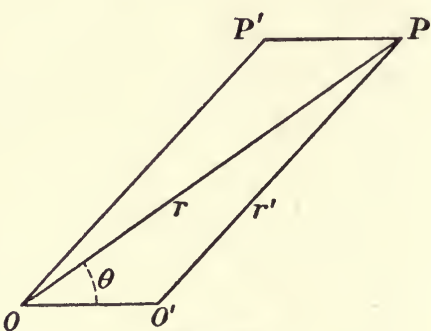

Fig. 72. is called the "strength." The resulting motion is evidently symmetrical about the axis.

If the direction $O O^{\prime}$ be that of the axis of $x$, and $O$ be taken as origin, the velocity-potential at $P$ due to simple sources $\pm m$ at $O^{\prime}$ and $O$, respectively, will be given by

$$
4 \pi \phi=m\left(\frac{e^{-i k r^{\prime}}}{r^{\prime}}-\frac{e^{-i k r}}{r}\right)
$$

where $r=O P, r^{\prime}=O^{\prime} P$. If we draw $P P^{\prime}$ equal and parallel to $O^{\prime} O$, we have $r^{\prime}=O P^{\prime}$, and the expression in brackets is equal to the change of value of the function $e^{-i k r} / r$ caused by a displacement of $P$ to $P^{\prime}$. Hence, ultimately, if $P^{\prime} P=\delta x$,

$$
4 \pi \phi=-m \delta x \cdot \frac{\partial}{\partial x}\left(\frac{e^{-i k r}}{r}\right) .
$$

Putting $m \delta x=1$, we deduce the formula for a unit double source at $O$, having its axis along $O x$, viz.

$$
4 \pi \phi=-\frac{\partial}{\partial x}\left(\frac{e^{-i k r}}{r}\right)
$$

this is a particular case of $\$ 73(3)$. When $x$ alone is varied, whilst $y$ and $z$ are constant, it appears from the figure that 
$\delta r=\cos \theta \delta x$, where $\theta$ denotes the inclination of $O P$ to $O x$. Hence $\partial / \partial x=\cos \theta \partial / \partial r$, and

$$
4 \pi \phi=-\frac{\partial}{\partial r}\left(\frac{e^{-i k r}}{r}\right) \cdot \cos \theta .
$$

Performing the differentiation, we find

$$
4 \pi \phi=\frac{(1+i k r) e^{-i k r}}{r^{2}} \cos \theta .
$$

For small values of $k r$, i.e. within distances from $O$ which are small compared with $\lambda / 2 \pi$, this becomes

$$
4 \pi \phi=\frac{\cos \theta}{r^{2}} \text {. }
$$

On the other hand, for large values of $k r$,

$$
4 \pi \phi=i k \frac{e^{-i k r}}{r} \cos \theta
$$

so that along any one radius vector the condensation $\left(s=\dot{\phi} / c^{2}\right)$ varies ultimately as $1 / r$. The radial and transverse components of the velocity are to be found by the formula (6) of $\$ 69$; viz. they are $-\partial \phi / \partial r$ and $-\partial \phi / r \partial \theta$, respectively. It appears that near the origin these are of the same order of magnitude, whilst at a great distance the lateral velocity is less than the radial in the ratio $1 / k r$.

Introducing the factor $C e^{i n t}$ in (24), and taking the real part, we find that the velocity-potential due to a double source, of strength $C \cos n t$, at a great distance, is

$$
\phi=-\frac{k C}{4 \pi r} \sin n\left(t-\frac{r}{c}\right) \cos \theta .
$$

The waves sent out in any direction $\theta$ are therefore ultimately plane, of the type ( 7 ), provided $A=k C \cos \theta / 4 \pi r$, the mere difference of phase being disregarded; and the flux of energy (across unit area) will therefore be $\rho k^{4} c C^{2} \cos ^{2} \theta / 32 \pi^{2} r^{2}$. Multiplying by $2 \pi r \sin \theta \cdot r \delta \theta$, which is the area of a zone of a spherical surface of radius $r$ bounded by the circles whose angular radii are $\theta$ and $\theta+\delta \theta$, and integrating from $\theta=0$ to $\theta=\pi$, we find that the total emission of energy by the double source $C \cos n t$ is

$$
W=\frac{\rho k^{4} c C^{2}}{24 \pi}
$$


It will be noticed that as the wave-length $\lambda$ is increased, and $k$ accordingly diminished, the fundamental equation (2) tends to assume the form

$$
\nabla^{2} \phi=0
$$

which is met with in the dynamics of incompressible fluids, and in the theories of attractions and of electric and thermal conduction. This assimilation may come about in two ways, either through a diminution in the frequency $(n / 2 \pi)$, or by an increase in the elasticity of the medium and consequently in the wavevelocity. Under the same condition the formula (12) approximates to the form

$$
\phi=\frac{A}{4 \pi r},
$$

which is the expression for the potential of a magnetic pole, or for a source of electricity, and so on; whilst in the case of the double source (21) the limiting form is (23), which is recognized as the potential of an infinitely small magnet.

A further remark of great importance is that within any region, free from sources, whose dimensions are small compared with $\lambda$, the configuration of the equipotential surfaces $\phi=$ const. is at any instant sensibly the same as if the fluid were incompressible. For the value of $\phi$ due to an external source differs from its value in the case of incompressibility chiefly by a factor $e^{-i k r}$, where $r$ denotes distance from the source. If $b$ denote the greatest breadth of the region, this factor can at most vary in the ratio $e^{-i k b}$, which differs very little from unity when $k b$ is small.

\section{Vibrating Sphere.}

By means of the fiction of a double source, of suitable strength, at the centre, it is possible to calculate the waves generated in the surrounding air by a vibrating solid sphere of any radius. As this is almost the only problem of the kind which can be completely solved we devote some space to it. The work is simple, and the results throw a good deal of light on other cases.

For reasons just referred to, it is instructive to look first at the case where the fluid is incompressible. We take the origin 
at the mean position of the centre of the sphere, and the axis of $x$ along the line of its vibration; and we denote its velocity by $U$. The velocity of the fluid in contact with the sphere at any point $P$, resolved in the direction of the normal, must be equal to the normal component of the velocity of the point $P$ of the sphere itself, i.e. to $U \cos \theta$, where $\theta$ is the angle $P O x$. This gives

$$
-\frac{\partial \phi}{\partial r}=U \cos \theta \quad[r=a]
$$

if $a$ be the radius. The velocity due to a double source at $O$ in an unlimited mass of incompressible fluid is of the form

$$
\phi=\frac{C}{4 \pi r^{2}} \cos \theta ;
$$

and in order that this may be consistent with (1) we must have

$$
C=2 \pi a^{3} U \text {. }
$$

With this determination of $C$ the effect of the sphere on the fluid is exactly that of the double source, and the solution of our problem is

$$
\phi=\frac{U a^{3}}{2 r^{2}} \cos \theta
$$

This depends only on the instantaneous value of $U$, as we should expect, since under the present hypothesis disturbances are propagated with infinite velocity. It should also be noted that there is so far no assumption that $U$ is small.

The directions of motion at various points of the field may be shewn by tracing the "lines of motion," which are lines drawn from point to point, always in the direction of the instantaneous velocity. In the case of small vibratory motion, which we have especially in view, each particle oscillates backwards and forwards through a short distance along the line on which it is situate. If $\delta r, r \delta \theta$ be the radial and transverse projections of an element of such a line, these quantities must be proportional to the radial and transverse components of velocity, viz. $-\partial \phi / \partial r$ and $-\partial \phi / r \partial \theta$, respectively. Hence

$$
\frac{\delta r}{\cos \theta}=\frac{r \delta \theta}{\frac{1}{2} \sin \theta}
$$

the integral of which is

$$
r^{2}=b^{2} \sin \theta
$$


where $b$ is a parameter which varies from one line of motion to another. The curves, which are identical in form with the lines of force due to a small magnet, are shewn in Fig. 73.

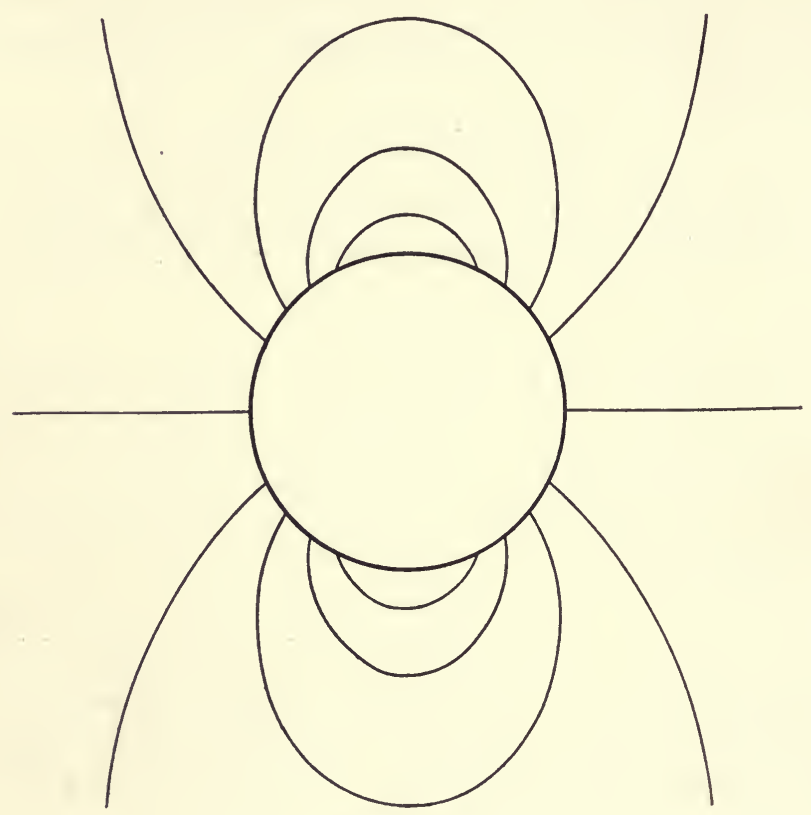

Fig. 73.

To calculate the reaction on the sphere we divide the surface into zones by planes perpendicular to $O x$. The area of a zone being $2 \pi a^{2} \sin \theta \delta \theta$, the resultant force on the sphere in the direction of $x$-positive is

$$
X^{\prime}=-\int_{0}^{\pi} p \cos \theta \cdot 2 \pi a^{2} \sin \theta d \theta
$$

The constant part of the pressure contributes nothing to the resultant. The variable part is, if terms of the second order in the velocities be neglected,

$$
p=\rho \frac{\partial \phi}{\partial t}=\frac{1}{2} \rho a \frac{d U}{d t} \cos \theta
$$


since $t$ enters only through $U$. Substituting we find

$$
X^{\prime}=-\frac{2}{3} \pi \rho a^{3} \frac{d U}{d t} .
$$

The remarkable point here is that the force is independent of the velocity, and depends only on the acceleration of the sphere. If the mass of the sphere be $M$, and if it be subject to other extraneous force $X$, its equation of motion will be

$$
\begin{gathered}
M \frac{d U}{d t}=X+X^{\prime}, \\
\left(M+\frac{2}{3} \pi \rho a^{3}\right) \frac{d U}{d t}=X .
\end{gathered}
$$

This is the same as if the fluid were abolished, and the inertia of the sphere were increased by $\frac{2}{3} \pi \rho a^{3}$, i.e. by half that of the fluid which it displaces. It was shewn by Stokes (1843) that this conclusion is accurate even when the restriction to small motions is abandoned.

There is, as we shall see ( $(79)$, nothing peculiar to the sphere in the general character of the above result, but the apparent addition to the inertia will vary of course with the shape as well as the size of the solid, and will usually be different for different directions of motion, as e.g. in the case of an ellipsoid. The theory here touched upon has had a great influence on recent physical speculations, and is responsible ultimately for the suggestion that the apparent inertia of ordinary matter may be partly or even wholly due to that of a surrounding aetherial medium.

Turning now to the acoustical problem, let the velocity of the sphere be expressed symbolically by

$$
U=A e^{i n t}
$$

The surface-condition will have the same form (1) as before. The velocity-potential of a double source $C e^{i n t}$ at $O$ is

$$
\phi=-\frac{C}{4 \pi} \frac{\partial}{\partial r}\left(\frac{e^{-i k r}}{r}\right) \cdot \cos \theta,
$$

by $\S 76(21)$, the time-factor $e^{\text {int }}$ being omitted. The ratio of $C$ to $A$ is then determined by (1). 
The most interesting case is where the radius $a$ of the sphere is small compared with $\lambda / 2 \pi$, where $\lambda$ is the wave-length. In the immediate neighbourhood of the sphere $k r$ will then be small, and the formula (13) is, for this region, practically identical with (2). It follows that

$$
C=2 \pi a^{3} A,
$$

nearly, and further that the lines of motion near the sphere will have sensibly the configuration shewn in Fig. 73. The apparent addition to the inertia of the sphere has very approximately the same value $\frac{2}{3} \pi \rho a^{3}$ as before. On the other hand, at distances $r$ which are comparable with, or greater than, $\lambda$, the motion of the fluid is altogether modified by the compressibility. At sufficiently great distances we have, by (13) and (14),

$$
\phi=\frac{1}{2} i k a^{3} A \frac{e^{-i k r}}{r} \cos \theta,
$$

or, in real form,

$$
\phi=-\frac{1}{2} \frac{k a^{3} A}{r} \sin n\left(t-\frac{r}{c}\right) \cos \theta
$$

corresponding to a velocity

$$
U=A \cos n t
$$

of the sphere. The amplitude now varies ultimately as $1 / r$, instead of $1 / r^{2}$, as in the case of (4).

The investigation so far discloses nothing analogous to a frictional resistance, whereas we know that owing to the generation of waves travelling outwards a continual abstraction of energy must take place. To calculate either the dissipative resistance, or the work done, at the surface of the sphere, we should have to use the complete formula (13); but the emission of energy may be ascertained independently from the formula (26) of $\$ 76$. The strength of the equivalent double source being given approximately by (14), we find

$$
W=\frac{1}{6} \pi \rho k^{4} a^{6} c A^{2} \text {. }
$$

If $\rho$ ' denote the mean density of the sphere, its energy when vibrating under the influence of (say) a spring will be

$$
\frac{1}{2} \cdot \frac{4}{3} \pi \rho^{\prime} a^{3} \cdot A^{2} \text {. }
$$


If, following a procedure explained in $§ 11$, we equate the rate of decay of this energy to $W$, we find

$$
\frac{d A}{d t}+\frac{A}{\tau}=0
$$

and therefore

$$
A=A_{0} e^{-t / \tau} \text {, }
$$

provided

$$
\tau=\frac{8}{n(k a)^{3}} \cdot \frac{\rho^{\prime}}{\rho} \text {. }
$$

The ratio $(n \tau / 2 \pi)$ of the modulus of decay to the period is therefore usually very great.

\section{Effect of a Local Periodic Force.}

Corresponding results can, with the help of more or less intuitive considerations, be obtained for other forms of vibrating solid, but the work is much simplified by a preliminary theorem, which has also an independent interest. This relates to the effect of a periodic extraneous force concentrated about a point in a gaseous medium.

An elementary proof can be derived at once from the preceding investigation. The result will obviously be the same if the force be imagined to act on an infinitely small sphere having the same density as the surrounding fluid. The effect is therefore that of a double source; and if we now denote the concentrated force, supposed acting parallel to $x$, by $P e^{i n t}$, we find, putting $M=\frac{4}{3} \pi \rho a^{3}$ in $\S 77$ (11),

$$
P=2 i \pi \rho k c a^{3} A,
$$

and therefore, by $\S 77$ (15), for large values of $k r$,

$$
\phi=\frac{P}{4 \pi \rho c} \frac{e^{-i k r}}{r}-\cos \theta .
$$

The following investigation is of a more formal character; but it involves mathematical processes more intricate than those which are employed in other parts of this book. The work depends on the solution of the equation

$$
\nabla^{2} \phi+k^{2} \phi=\Phi,
$$

where $\Phi$ is a given function of $x, y, z$ which vanishes outside a certain finite region $R$. In the theories of attraction, and of thermal and electric conduction, we meet with the equation

$$
\nabla^{2} \phi=\Phi,
$$


where $\Phi$ represents a distribution of density $(\rho=-\Phi / 4 \pi)$, or of sources of heat, \&c. The solution of (4) appropriate to infinite space (when there are no sources at infinity) is known, viz. it is

$$
\phi=-\frac{1}{4 \pi} \iiint \frac{\Phi^{\prime}}{r} d x^{\prime} d y^{\prime} d z^{\prime}
$$

where $\Phi^{\prime}$ denotes the value of $\Phi$ at $\left(x^{\prime}, y^{\prime}, z^{\prime}\right), r$ denotes distance from this point to the point $P$, or $(x, y, z)$, for which the value of $\phi$ is required, and the integration extends over all space for which $\Phi$ differs from 0 . For example, if we put $\Phi^{\prime}=-4 \pi \rho^{\prime}$, we get the ordinary expression for the gravitation potential of a continuous distribution of matter.

The analogous solution of (3) is

$$
\phi=-\frac{1}{4 \pi} \iiint \Phi^{\prime} \frac{e^{-i k r}}{r} d x^{\prime} d y^{\prime} d z^{\prime}
$$

This represents a distribution of simple sources through $R$, the strength per unit volume being $\Phi$, and it is therefore obvious at once that the equation $\nabla^{2} \phi+k^{2} \phi=0$ is satisfied at all points $P$ external to $R$. The only question of any difficulty arises when $P$ is inside $R$. We then divide $R$ into two regions $R_{1}$ and $R_{2}$, of which $R_{2}$ encloses $P$ and is ultimately taken to be infinitely small in all its dimensions. The parts of $\phi$ at $P$ due to the sources in $R_{1}$ and $R_{2}$, respectively, may be denoted by $\phi_{1}$ and $\phi_{2}$. Since $P$ is external to $R_{1}$ we have $\nabla^{2} \phi_{1}+k^{2} \phi_{1}=0$ as before. Within $R_{2}$ we may ultimately put $e^{-i k r}=1$, and $\phi_{2}$ then approximates to the gravitation potential of matter of density - $\Phi / 4 \pi$ restricted to the space $R_{2}$. We have then, ultimately, on known principles, $\nabla^{2} \phi_{2}=\Phi$ and $\phi_{2}=0$. Hence (1) is satisfied by $\phi=\phi_{1}+\phi_{2}$. It is further evident that (6) is the solution of (1) consistent with the condition that there are no sources of sound except at the points to which $\Phi$ refers.

When forces $X, Y, Z$ per unit mass act at the various points of a gaseous medium, the equations (4) of $\S 68$ are replaced by

$$
\frac{\partial u}{\partial t}=-c^{2} \frac{\partial s}{\partial x}+X, \frac{\partial v}{\partial t}=-c^{2} \frac{\partial s}{\partial y}+Y, \frac{\partial w}{\partial t}=-c^{2} \frac{\partial s}{\partial z}+Z .
$$


If we eliminate $u, v, w$ by the kinematical relation (1) of $\$ 70$ we obtain

$$
\frac{\partial^{2} s}{\partial t^{2}}=c^{2} \nabla^{2} s-\left(\frac{\partial X}{\partial x}+\frac{\partial Y}{\partial y}+\frac{\partial Z}{\partial z}\right) .
$$

If $X, Y, Z, s$ all vary as $e^{i k c t}$, this becomes

$$
\nabla^{2} s+k^{2} s=\frac{1}{c^{2}} \operatorname{div}(X, Y, Z),
$$

in the notation of $\S 67$. This is of the form (3), and the solution is therefore

$$
\begin{aligned}
s & =-\frac{1}{4 \pi c^{2}} \iiint\left(\frac{\partial X^{\prime}}{\partial x^{\prime}}+\frac{\partial Y^{\prime}}{\partial y^{\prime}}+\frac{\partial Z^{\prime}}{\partial z^{\prime}}\right) \frac{e^{-i k r}}{r} d x^{\prime} d y^{\prime} d z^{\prime} \\
& =\frac{1}{4 \pi c^{2}} \iiint\left(X^{\prime} \frac{\partial}{\partial x^{\prime}}+Y^{\prime} \frac{\partial}{\partial y^{\prime}}+Z^{\prime} \frac{\partial}{\partial z^{\prime}}\right) \frac{e^{-i k r}}{r} d x^{\prime} d y^{\prime} d z^{\prime} .
\end{aligned}
$$

The transformation is effected by partial integration of the several terms, the integrated portions vanishing at infinity if $X^{\prime}, Y^{\prime}, Z^{\prime}$ do so. Also, since

we have

$$
r=\sqrt{ }\left\{\left(x-x^{\prime}\right)^{2}+\left(y-y^{\prime}\right)^{2}+\left(z-z^{\prime}\right)^{2}\right\},
$$

whence

$$
\partial / \partial x^{\prime}=-\partial / \partial x, \quad \partial / \partial y^{\prime}=-\partial / \partial y, \quad \partial / \partial z^{\prime}=-\partial / \partial z,
$$

$$
s=-\frac{1}{4 \pi c^{2}} \iiint\left(X^{\prime} \frac{\partial}{\partial x}+Y^{\prime} \frac{\partial}{\partial y}+Z^{\prime} \frac{\partial}{\partial z}\right) \frac{e^{-i k r}}{r} d x^{\prime} d y^{\prime} d z^{\prime} .
$$

From this the value of $\phi$ follows by the relation

$$
c^{2} s=\frac{\partial \phi}{\partial t}=i n \phi=i k c \phi \text {. }
$$

As a particular case, suppose that $Y^{\prime}=0, Z^{\prime}=0$, and that $X^{\prime}$ differs from 0 only in a small region about the origin, and put

$$
\rho \iiint X^{\prime} d x^{\prime} d y^{\prime} d z^{\prime}=P \text {. }
$$

We have

$$
\phi=\frac{i P}{4 \pi \rho k c} \frac{\partial}{\partial x}\left(\frac{e^{-i k r}}{r}\right),
$$

or, for large values of $k r$,

$$
\phi=\frac{P}{4 \pi \rho c} \frac{e^{-i k r}}{r} \cos \theta,
$$

as before. Comparing with $\$ 76$ (24) we see that a concentrated force $P e^{i n t}$ has the effect of a double source of strength $-i P / \rho k c$. 
79. Waves generated by Vibrating Solid.

We return to the problem of investigating the waves generated by a vibrating body. In order not to complicate the question too much we will assume that the body has some sort of symmetry with respect to an axis; thus it may be a form of revolution about this axis, or it may have two mutually perpendicular planes of symmetry meeting in this axis, or (again) a single plane of symmetry perpendicular to the axis. In any case this axis is taken to be the direction $(O x)$ of vibration.

The dimensions of the solid being supposed small in comparison with $\lambda / 2 \pi$, the motion of the fluid in the immediate neighbourhood will be sensibly the same as in the case of incompressibility, and the principal effect on the body will therefore be equivalent to an increase of inertia. To establish this latter point in a general manner, we note that the (irrotational) motion of a frictionless liquid due to the motion of a solid in it will have the velocity at every point in a determinate ratio to the velocity $U$ of the solid, and that the total kinetic energy of the fluid may therefore be expressed by $\frac{1}{2} \rho Q^{\prime} U^{2}$, where $Q^{\prime}$ is a constant, of the nature of a volume, depending only on the size and shape of the solid and the direction of its vibration. Hence if $M$ be the mass of the body, the equation of energy takes the form

$$
\frac{d}{d t}\left(\frac{1}{2} M U^{2}+\frac{1}{2} \rho Q^{\prime} U^{2}\right)=X U,
$$

where the right-hand member represents the rate at which work is being done by the extraneous force $X$. Thus

$$
\left(M+\rho Q^{\prime}\right) \frac{d U}{d t}=X,
$$

which shews that the inertia of the body is apparently increased by the amount $\rho Q^{\prime}$. An equivalent statement is that the reaction of the liquid is equivalent to a force $-\rho Q^{\prime} d U / d t$.

In the actual case of the gaseous medium, it is plain that if the solid were removed, and its place supplied by fluid, the motion at a distance would be very approximately the same as would be produced by a suitable periodic force from without, 
acting on the substituted matter. Since this force has to produce an acceleration of momentum $\rho Q d U / d t$, where $Q$ is the volume displaced by the solid, as well as to balance the reaction just referred to, its amount would be

$$
P=\rho\left(Q+Q^{\prime}\right) \frac{d U}{d t}=i n \rho\left(Q+Q^{\prime}\right) A e^{i n t},
$$

if

$$
U=A e^{i n t}
$$

By $\$ 78$ (2), the velocity-potential at a great distance $r$ will therefore be

$$
\phi=\frac{P}{4 \pi \rho c} \frac{e^{-i k r}}{r} \cos \theta=\frac{i k\left(Q+Q^{\prime}\right)}{4 \pi r} A e^{i k(c t-r)} \cos \theta .
$$

Comparing with $\S 76$ (24) we see that the effect of the vibrating solid is equivalent to a double source of strength $C=\left(Q+Q^{\prime}\right) A$, and that the emission of energy is accordingly

$$
W=\frac{\rho k^{4} c}{24 \pi}\left(Q+Q^{\prime}\right)^{2} A^{2},
$$

by $\$ 76$ (26). In the case of the sphere we have $Q=\frac{4}{3} \pi a^{3}$, $Q^{\prime}=\frac{1}{2} Q$, and the result accordingly agrees with $\S 77$ (18). It can be shewn that for a circular disk of radius $a$, moving broadside on, $Q^{\prime}=\frac{8}{3} \pi a^{3}$, whilst $Q$ of course $=0$.

\section{Communication of Vibrations to a Gas.}

The circumstances which govern the efficiency of a vibrating body in generating sound waves, and the comparative effects in different gases, were elucidated by Stokes in a classical memoir "On the Communication of Vibrations from a Vibrating Body to a surrounding Gas*." The starting point of the investigation was an observation by Prof. J. Leslie (1837), who found that the sound emitted by a bell vibrating in an atmosphere of hydrogen was extremely feeble as compared with the effect in air. No satisfactory explanation of this phenomenon was forthcoming up to the time of Stokes' paper. The essence of the matter is conveyed in the following quotation:

"When a body is slowly moved to and fro in any gas, the gas behaves almost exactly like an incompressible fluid, and

* Phil. Trans. 1868. The passage which follows below is from the "abstract" in the Proc. Roy. Soc. 
there is merely a local reciprocating motion of the gas from the anterior to the posterior region, and back again in the opposite phase of the body's motion, in which the region that had been anterior becomes posterior. If the rate of alternation of the body's motion be taken greater and greater, or, in other words, the periodic time less and less, the condensation and rarefaction of the gas, which in the first instance was utterly insensible, presently becomes sensible, and sound waves (or waves of the same nature in case the periodic time be beyond the limits of audibility) are produced, and exist along with the reciprocating flow. As the periodic time is diminished, more and more of the encroachment of the vibrating body on the gas goes to produce a true sound wave, less and less a mere local reciprocating flow. For a given periodic time, and given size, form, and mode of vibration of the vibrating body, the gas behaves so much the more nearly like an incompressible fluid as the velocity of propagation of sound in it is greater; and on this account the intensity of the sonorous vibrations excited in air as compared with hydrogen may be vastly greater than corresponds merely with the difference of density of the two gases."

These remarks are exemplified in the results of $\S 77$ (13), (14). If we fix our attention on a point at a distance from the sphere, supposed vibrating with the velocity

$$
U=A \cos n t,
$$

the motion there is given, when the period is sufficiently long, by the formula

$$
\phi=\frac{A a^{3}}{2 r^{2}} \cos \theta \cdot \cos n t,
$$

as if the fluid were incompressible. But when the frequency is increased until the wave-length is small compared with the distance $r$ from the centre, the appropriate formula is

$$
\phi=-\frac{k a^{3} A}{2 r} \cos \theta \cdot \sin n\left(t-\frac{r}{c}\right),
$$

and the amplitude is accordingly greater than in the former case in the ratio $k r$, or $2 \pi r / \lambda$. For the same frequency, the amplitude, which depends on $k / c$ or $n / c^{2}$, will in different gases 
now vary inversely as the square of the wave-velocity. Again, the emission of energy is, by $\$ 77$ (17),

$$
W=\frac{1}{6} \pi \rho k^{4} a^{6} c A^{2}=\frac{1}{6} \pi \rho n^{4} a^{6} / c^{3} \cdot A^{2},
$$

and so varies (for the same gas) as the fourth power of the frequency. The emission in different gases will (for the same frequency) vary inversely as the fifth power of the wavevelocity, if we assume ( $(59)$ that the latter varies inversely as the square root of the density. For instance it will be about 1000 times less in hydrogen than in oxygen.

In order further to illustrate the effect of the lateral motion of the gas, near the surface of the sphere, from the hemisphere which is at the moment moving outwards to that which is moving inwards, in weakening the intensity of the waves propagated to a distance, we may calculate what the emission would be if this lateral motion were prevented. For this purpose we may (after Stokes) imagine a large number of fixed partitions to extend radially outwards from near the surface. In any one of the narrow conical tubes thus formed, the motion will be of the same character as in the case of symmetrical spherical waves. Now a uniform radial velocity $C \cdot \cos n t$ over the surface of a sphere would be equivalent to a simple source $4 \pi a^{2} C \cos n t$, and the corresponding emission per unit area would be $\frac{1}{2} k^{2} a^{2} \rho c C^{2}$, by $\S 76(15)$. If we now put $C=A \cos \theta$, and integrate over the surface, we get the total emission in our system of conical tubes. The result is

$$
W^{\prime}=\frac{2}{3} \pi k^{2} a^{4} \rho c A^{2},
$$

since the average of $\cos ^{2} \theta$ for all directions in space is $\frac{1}{3}$. If we compare this with (4), we see that the effect of the lateral motion is to diminish the emission in the ratio $\frac{1}{4} k^{2} a^{2}$.

When, as for example in the case of a plate or a bell, the surface is divided by nodal lines into a number of compartments vibrating in opposite phases, the opportunity of lateral motion is increased, and the emission of energy correspondingly weakened. For facility of calculation Stokes took the case of a spherical surface, with various symmetrical arrangements of nodal lines. In the problem of the oscillating sphere we have one such line, viz. the great circle $\theta=\frac{1}{2} \pi$, and the emission, as 
we have just seen, is diminished by the lateral motion in the ratio $\frac{1}{4} k^{2} a^{2}$. For a spherical surface with two nodal great circles meeting at right angles the effect is much greater, the ratio being $\frac{1}{81} k^{4} a^{4}$. And as we increase the number of compartments into which the sphere is divided, the ratio, already very small, decreases with enormous rapidity.

For the sake of simplicity it has been assumed in the preceding statements that the perimeter $2 \pi a$ of the sphere is small compared with $\lambda$. The influence of lateral motion is however not confined to this case, but will make itself felt whenever the dimensions of the compartments referred to are small compared with $\lambda$. In the case of the oscillating sphere there is no difficulty in working out the result without any restriction to the value of $k a$, starting from the formula (13) of $\$ 77$.

Stokes has also investigated mathematically the case of a cylinder vibrating at right angles to its length, where the same cause is of course operative. In this way an estimate is obtained of the direct effect of a vibrating string in generating air-waves. This involves the ratio of the perimeter of the cross-section of the string to the length of the air-waves, and is in any practical case extraordinarily minute. As explained in $\S 24$, almost the whole of the sound given out when a piano string is struck comes from the sounding board.

\section{Scattering of Sound Waves by an Obstacle.}

We have next to consider the disturbance produced in a train of sound waves by a rigid obstacle whose dimensions are small compared with the wave-length. The scattered waves which are sensible at a distance are due mainly to two causes. If the obstacle were absent the space which it occupies would be the seat of alternate condensations and rarefactions. The effect of the obstacle in refusing to execute the corresponding contractions and expansions of volume is, at a distance, approximately the same as if in a medium otherwise at rest its volume were to undergo a periodic change of just the opposite character. The result is equivalent to a simple source. On the disturbance thus produced there is superposed a second 
wave-system, which is due to the immobility of the obstacle. If the latter were freely movable, and if it had moreover the same density as the surrounding air, it would swing to and fro with the air-particles, and the second wave-system would be absent. This system is accordingly the same as would be produced if the obstacle were constrained to oscillate with a motion exactly equal and opposite to that of the air in the primary waves when undisturbed. The effect is, as we have seen in $\$ 79$, that of a double source. It might appear, at first sight, that the former of these disturbing influences would be much less important than the second, but in its effect at a distance it becomes comparable, owing to the greater attenuation by lateral motion of the waves proceeding from a double source.

If $Q$ be the volume of the obstacle, the strength of the simple source due to the first cause is

$$
-Q \frac{\partial s}{\partial t}=-\frac{Q}{c^{2}} \frac{\partial^{2} \phi}{\partial t^{2}}=k^{2} Q \phi
$$

where $s, \phi$ refer to the primary waves. In the case of a system of plane waves

$$
\phi=C e^{-i k x}
$$

incident on a small obstacle at $O$, this gives a velocity-potential

$$
\phi_{1}=\frac{k^{2} Q C}{4 \pi r} e^{-i k r} .
$$

As regards the second cause, we will assume for simplicity that the obstacle has the degree of symmetry postulated in $\S 79$ with respect to the direction $(O x)$ of the vibration in the air-waves. If the wave-system (2) were undisturbed, the velocity of the air-particles at $O$ would be represented symbolically by $i k C$, and the strength of the double source due to the obstacle moving with this velocity reversed would be $-i k\left(Q+Q^{\prime}\right) C$, in the notation of $\S 79$. The scattered waves at a distance, due to the immobility, are therefore represented by

$$
\phi_{2}=\frac{k^{2}\left(Q+Q^{\prime}\right) C}{4 \pi r} e^{-i k r} \cos \theta,
$$

by $\$ 76$ (24). The complete result is given by $\phi=\phi_{1}+\phi_{2}$.

It follows that the amplitude of the scattered waves at any

L. 
distant point is, for similar forms, directly proportional to the volume of the obstacle and inversely proportional to the square of the wave-length. This latter particular might have been foreseen without calculation. The ratio to the original amplitude must necessarily vary directly as the volume $Q$, and inversely as the distance $r$, and in order that the result may come out a pure number we must divide by $\lambda^{2}$, since $\lambda$ is the only other linear magnitude involved. The emission of energy, being proportional to the square of the amplitude, will therefore vary as $\lambda^{-4}$. This law of the inverse fourth power holds also in optics, and for a similar reason, with respect to the scattering of light by particles whose dimensions are small compared with the dimensions of the light-waves. The blue of the sky, for instance, is attributed to the relative preponderance of the shorter waves in the light scattered by the molecules of air, and possibly by other particles; in the transmitted light, on the other hand, the longer waves predominate. The theory is due to Lord Rayleigh, who has also pointed to an acoustic illustration in what are called "harmonic echoes." If a composite musical note, consisting of a fundamental tone with its octave, \&c., be sounded near a grove of trees, for example, the ratio of the intensity of the octave to that of the fundamental will in the scattered sound be 16 times what it was in the original note. The scattered sound may therefore appear to be raised in pitch by an octave.

The actual scattering of energy is found by adding the results due to the simple and the double source. This may be proved by calculating the work done at the surface of a sphere of large radius $r$. The terms due to the combined action of the two sources contain a factor $\cos \vartheta$, and so disappear when integrated over the surface. Hence, by $\S 76$ (15), (26),

$$
W=\frac{\rho k^{6} c C^{2}}{8 \pi}\left\{Q^{2}+\frac{1}{3}\left(Q+Q^{\prime}\right)^{2}\right\}
$$

The energy-flux in the primary waves being $\frac{1}{2} \rho k^{2} c C^{2}$, by $\S 76$ (8), the ratio which the energy scattered per second bears to this is

$$
\frac{k^{4}}{4 \pi}\left\{Q^{2}+\frac{1}{3}\left(Q+Q^{\prime}\right)^{2}\right\}
$$


In the case of the sphere we found $Q^{\prime}=\frac{1}{2} Q=\frac{2}{3} \pi a^{3}$, and the expression (6) therefore reduces to

$$
\frac{7}{9}(k a)^{4} \cdot \pi a^{2} \text {. }
$$

In other words, the sphere scatters only the fraction $\frac{7}{9}(k a)^{4}$ of the energy which falls upon it. For example, if the wavelength be a metre (which corresponds to a frequency of about 332 ), and the diameter of the sphere $1 \mathrm{~mm}$., the fraction is roughly $7.6 \times 10^{-11}$. In the case of the circular disk, where $Q^{\prime}=\frac{8}{3} \pi a^{3}, Q=0$, the ratio of the scattered to the incident energy is $\frac{16}{27}(k a)^{4}$.

The mathematical theory of the scattering by cylindrical obstacles is more difficult. We will merely quote the result, based on Lord Rayleigh's calculations, that when plane waves are incident on a circular cylinder of radius $a$ the fraction of the incident energy which is scattered is $\frac{3}{8} \pi^{2}(k a)^{3}$, approximately, it being assumed as usual that $k a$ is small. For a wire of diameter $1 \mathrm{~mm}$., and a wave-length of a metre, this

$$
=1 \cdot 15 \times 10^{-7} \text {. }
$$

It is to be observed however that in the case of very minute obstacles the order of magnitude of the results may be considerably modified by viscosity. The determining element here is the ratio of the diameter of the obstacle to the quantity $h$ which was introduced in $\S 66$ as a measure of the thickness of the air-stratum, at the surface of the obstacle, whose motion is appreciably affected by the friction. When the ratio in question is moderately large the influence of viscosity on the results will be very slight.

The distribution of velocity in the immediate neighbourhood of the obstacle will be sensibly the same as in the case of a uniform current of incompressible fluid flowing past the body. In the case of the sphere it can be determiner completely, but the following approximation will be sufficient. We assume

$$
\phi=C e^{-i k r \cos \theta}+\frac{B}{r^{2}} \cos \theta,
$$

where the first term represents the incident waves, and the 
second is the form which the velocity-potential of a double source assumes $(\S 76)$ when $k r$ is small. This makes

$$
-\frac{\partial \phi}{\partial r}=i k C \cos \theta e^{-i k r}+\frac{2 B}{r^{3}} \cos \theta,
$$

and the condition of zero normal velocity for $r=a$ is therefore approximately satisfied provided $B=-\frac{1}{2} i k a^{3} C$. Hence in the neighbourhood of the sphere we have

$$
\phi=C\left\{1-i k\left(r+\frac{a^{3}}{2 r^{2}}\right) \cos \theta\right\},
$$

nearly. The velocities are therefore nearly the same as if the fluid were incompressible. The pressure is given by

$$
p=p_{0}+\rho \dot{\phi}=p_{0}+i n \rho \phi .
$$

This differs from the pressure $\left(p_{0}+i n \rho C\right)$ which would obtain at the origin if the obstacle were absent by a term which is small, of the order $k r$, in comparison. At points whose distance $r$ is a moderate multiple of $a$, whilst still small compared with $\lambda$, the pressure approximates even more closely to that due to the incident waves alone.

\section{Transmission of Sound by an Aperture.}

In discussing the transmission of sound waves by an aperture in a thin screen we will suppose, in the first instance, that the dimensions of the aperture are small compared with the wavelength. This is of course the most interesting case from an acoustical point of view.

The screen being supposed to occupy the plane $x=0$, and the origin being taken in the aperture $(S)$, let a wave-train represented by

$$
\phi=C e^{-i k x}
$$

be incident from the left. If we distinguish the functions relating to the two sides of the screen by the suffixes 1 and 2 , we should have, if the screen were complete,

$$
\phi_{1}=C e^{-i k x}+C e^{i k x}, \quad \phi_{2}=0,
$$

the second term in $\phi_{1}$, which represents reflected waves, being chosen so as to make $\partial \phi_{1} / \partial x=0$ for $x=0$.

In the actual problem the disturbance due to the aperture will be confined mainly to the immediate neighbourhood of $S$, and may be taken to be very small at distances from $O$ which, 
though large as compared with the linear dimensions of $S$, are small compared with $\lambda$. Let two surfaces be drawn, on the two sides, at some such distance from 0 , each abutting on the screen in the manner indicated by the dotted lines in the figure. Within the region thus bounded, the fluid oscillates backwards and forwards almost as if it were incompressible, and the total flux ( $§ 67)$ through the aperture will therefore bear a constant ratio to the difference of the velocity-potentials at the two sarfaces. This will perhaps be understood more clearly if we have recourse to the analogy of electric conduction. Suppose we have a large metallic mass, severed almost in two by a non-conducting partition occupying the place of the screen. If this mass form part of an electric circuit, there will be little variation of potential in it except in the neighbourhood of the narrow neck which connects the two portions. The electric potentials at a distance on the two sides being

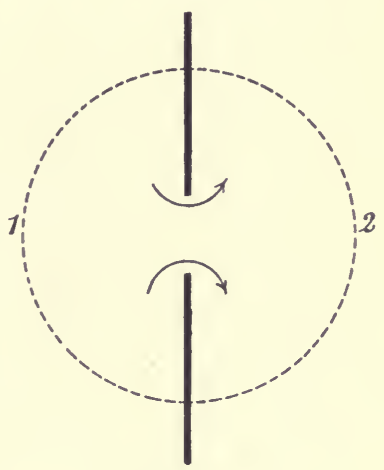

Fig. 74 . $\phi_{1}$ and $\phi_{2}$, the current through the neck will be

$$
K\left(\phi_{1}-\phi_{2}\right) \text {, }
$$

where $K$ may be called the "conductivity" of the neck, the specific conductivity of the substance being taken to be- unity. In the hydrodynamical question, also, the quantity $K$ may appropriately be called the conductivity of the aperture. It is easily seen that it is of the nature of a length.

At the two surfaces shewn in the figure we have $\phi_{1}=2 C$, $\phi_{2}=0$, approximately, and the total flux through the aperture is therefore $2 K C$. If an equal flux were directed symmetrically from the aperture on the left-hand side, the combination would be equivalent, in an unlimited medium, to a simple source of strength $4 K C$. Hence, by $\$ 76$ (12),

$$
\phi_{2}=\frac{K C}{\pi r} e^{-i k r} .
$$


The corresponding velocity-potential on the near side is evidently

$$
\phi_{1}=C e^{-i k x}+C e^{i k x}-\frac{K C}{\pi r} e^{-i k r} .
$$

The energy $(W)$ transmitted by the aperture per second is by the above reasoning one-half that due to a simple source $4 K C$ at $O$, whence, by $\S 76(15)$,

$$
W=\rho k^{2} c K^{2} C^{2} / \pi \text {. }
$$

The energy-flux in the primary waves (1) being $\frac{1}{2} \rho k^{2} c C^{2}$, the ratio of $W$ to this is $2 K^{2} / \pi$. It is to be noted that this is independent of the wave-length $\lambda$, so long, of course, as $\lambda$ is large compared with the linear dimensions of $S$.

The exact calculation of $K$ for various forms of aperture is naturally a matter of some difficulty. For a circular aperture of radius $a$ it is found that $K=2 a$; for other forms differing little from a circle the value is sensibly the same as for a circular aperture of the same area, the circle being evidently a "stationary" form, in the sense in which this term is used in the theory of maxima and minima. It appears then that a circular (or nearly circular) aperture transmits the fraction $8 / \pi^{2}$, or 816 , of the energy propagated across an equal area $\left(\pi a^{2}\right)$ in the primary waves. This is, under the present limitation as to size, very great compared with the energy intercepted by a disk of the same dimensions ( $\$ 81)$. The figure opposite gives the shapes of the surfaces of equal pressure $(\phi=$ const.), drawn for equidistant values of $\phi$, in the immediate neighbourhood of a circular aperture, and shews how rapidly these tend to assume the spherical form. The directions of vibration of the air-particles are of course normal to these surfaces.

With regard to further problems of the kind we must content ourselves with a few statements of results. In the case of an aperture in the shape of a long narrow slit, whose breadth is small compared with $\lambda$, the energy transmitted is again comparable with, and may even considerably exceed, that corresponding to an equal area of wave-front in the primary waves. In the case of a grating composed of equal, 
parallel, and equidistant slits in a thin screen, the fraction of the total incident energy which is transmitted is found to be $1 /\left(1+k^{2} l^{2}\right)$, where $k=2 \pi / \lambda$, as usual, and

$$
l=\frac{a+b}{\pi} \log \sec \frac{\pi b}{2(a+b)},
$$

where $a$ denotes the breadth of an opening, and $b$ that of each intervening portion of the screen. As a numerical example,

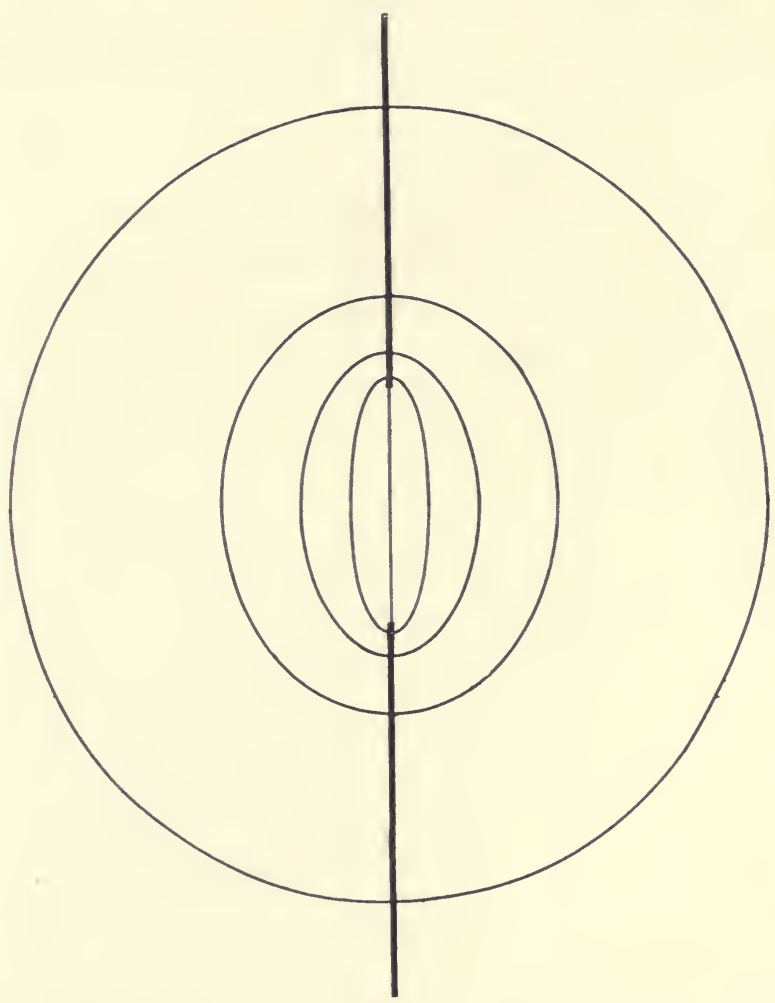

Fig. 75 .

suppose the wave-length to be ten times the interval $a+b$ between the centres of successive apertures; then even if the apertures form only one-tenth part of the whole area of the screen, 88 per cent. of the sound will get through. In the 
case of a grating formed by equidistant bars of circular section, the corresponding value of $l$ is

$$
l=\pi b^{2} / a,
$$

where $b$ is the radius of the section, and $a$ the distance between the axes of consecutive bars. It is implied, however, that the ratio $b / a$ must not exceed (say) $\frac{1}{4}$.

\section{Contrast between Diffraction Effects in Sound and}

\section{Light. Influence of Wave-Length.}

In the investigation of $\S 82$ an aperture was found to act as a simple source from which sound diverges on the farther side uniformly in all directions. This is in striking contrast with what is usually observed in the case of light. We have so far no indication of anything of the nature of beams or rays of sound, just as when sound waves were incident on an obstacle we found nothing of the nature of a sound-shadow. The difference in the results is due to the fact that the dimensions of the aperture (or obstacle) have been supposed small in comparison with the wave-length, whereas with light the relation is usually the reverse.

We have avoided trespassing on the domain of Optics, but as the dynamical conditions are in the present subject perfectly definite, it may be permissible to examine this question of the influence of wave-length a little more fully.

Consider the region of space lying to the right of the plane $x=0$. If this plane were a fixed boundary, and if there were no sources of sound in the region, any disturbance would ultimately pass away. Any steady periodic motion in the region must therefore in the absence of internal sources be due to motion of the boundary, and will be determinate when the value of the normal component of the velocity at every point of the latter is given. It can, moreover, be expressed in terms of this distribution of normal velocity, as follows. The flux outwards from an element $\delta S$ of the plane is $-\partial \phi / \partial n . \delta S$, if $\delta n$ denote an element of the normal drawn inwards from $\delta S$, and if in imagination we associate with this an equal flux in the opposite direction on the other side, the result is equivalent 
to a source $-2 \partial \phi / \partial n . \delta S$ in infinite space. The corresponding velocity-potential at a point $P$ is

$$
-\frac{1}{2 \pi} \frac{\partial \phi}{\partial n} \delta S \cdot \frac{e^{-i k r}}{r}
$$

where $r$ denotes the distance of $\delta S$ from $P$. Integrating over all the elements $\delta S$ of the plane, we have

$$
\phi_{P}=-\frac{1}{2 \pi} \iint \frac{\partial \phi}{\partial n} \frac{e^{-i k r}}{r} d S
$$

which is the required formula.

The motion to the right of the plane $x=0$ is also determinate when the value of $\phi$ at every point of the plane, and thence the pressure, is given, these two quantities being connected by the relation $p=p_{0}+\rho \dot{\phi}=p_{0}+i k c \rho \phi$. Suppose for a moment that in an otherwise unlimited medium we have a thin massless membrane occupying the plane $x=0$, and that on each element of this a normal force $X$ per unit area is exerted, which is adjusted so as to produce the actual periodic pressure, and therefore the actual value of $\phi$, on the positive face of the membrane. By the theorem of $\S 78$ (15), the effect for an element $\delta S$, will be equivalent to a double source, and the corresponding velocity-potential at a point $P$ will be

$$
\frac{i}{4 \pi k c \rho} X \delta S \cdot \frac{\partial}{\partial x}\left(\frac{e^{-i k r}}{r}\right) \text {. }
$$

The variable parts of the pressures on the two faces of the membrane, viz. $\pm \rho \dot{\phi}= \pm i k c \rho \phi$, must balance the force $X$, so that $X=2 i k c \rho \phi$. Substituting in (2), and integrating over the plane $x=0$, we obtain

$$
\phi_{P}=-\frac{1}{2 \pi} \iint \phi \frac{\partial}{\partial x}\left(\frac{e^{-i k r}}{r}\right) d S .
$$

The structure of the integrals in (1) and (3) recalls the process by which "Huygens' principle" is applied in optics to find the disturbance at any point $P$ in terms of "secondary waves" supposed to issue from the various elements of a wave-front. There was at one time much discussion as to the exact character to be assigned to these secondary waves, more especially as to the law of intensity in different directions. We now recognize 
that the problem has mathematically more than one solution; either of the above formulae will lead to an exact result, and we might even use a combination of the two, in any arbitrary proportions. This resolution of a historic controversy is due to Lord Rayleigh.

As a verification of (3), suppose that the value of $\phi$ at $x=0$ is that due to a train of plane waves $\phi=e^{-i k x}$. Let $\approx$ denote the distance of $\delta S$ from the orthogonal projection of the point $P$ on the plane $x=0$, so that $r^{2}=x^{2}+\varpi^{2}$. For the aggregate of elements $\delta S$ forming a certain annulus of the plane we may write $2 \pi \varpi \delta \varpi=2 \pi r \delta r$. We have also $\partial r / \partial x=x / r$. The formula (3) therefore gives

$$
\begin{aligned}
\dot{\phi}_{P}=-\frac{1}{2 \pi} \int_{0}^{\infty} \frac{\partial}{\partial x}\left(\frac{e^{-i k r}}{r}\right) 2 \pi \varpi d \varpi & \\
& =-x \int_{x}^{\infty} \frac{\partial}{\partial r}\left(\frac{e^{-i k r}}{r}\right) d r=e^{-i k x}
\end{aligned}
$$

In the case of waves transmitted by an aperture in a plane screen $(x=0)$, we have, in (1), $\partial \phi / \partial n=0$ except over the area of the aperture. If further, the dimensions of the aperture $S$ are small compared with $\lambda$, then at a point $P$ whose distance $r$ is large compared with $\lambda$, the function $e^{-i k r} / r$ will have sensibly the same value for all the elements of $S$, and we may write

$$
\phi_{P}=-\iint \frac{\partial \phi}{\partial n} d S \cdot \frac{e^{-i k r}}{2 \pi r},
$$

where the first factor represents the total flux through $S$. Under these circumstances the aperture acts like a simple source, as in $\S 82$.

It is understood of course that the expression $-\partial \phi / \partial n$ in (1) or (5) represents the normal component of the velocity, as modified by the action of the screen. When as in the case just considered the aperture is relatively small, the distribution of normal velocity over it will differ considerably from that due to the primary waves alone. This distribution can be ascertained approximately, in the case of plane waves incident directly on a circular opening, from the electrical analogy of $\S 82$. The lines of flow have the same configuration as the lines of force 
due to an electrified disk*, and the normal velocity has the distribution

$$
-\frac{\partial \phi}{\partial n}=\frac{B}{\sqrt{\left(a^{2}-\varpi^{2}\right)}}
$$

where $\varpi$ denotes the distance of any point of the aperture from its centre. The velocity becomes very great near the edge, and is mathematically infinite + at the edge itself $(\varpi=a)$, but it appears on integration that the parts of the area near the edge contribute little to the total flux, which is

$$
-\int_{0}^{a} \frac{\partial \phi}{\partial n} 2 \pi \varpi d \varpi=2 \pi a B .
$$

If the incident waves be represented by

$$
\phi=C e^{-i k x} \text {, }
$$

the same flux will as in $\S 82$ be expressed by $2 K C$, or $4 a C$. Hence, comparing,

$$
B=2 / \pi . C \text {. }
$$

In the other extreme, where the wave-length is only a minute fraction of the dimensions of the aperture, the effect of the screen in modifying the distribution of normal velocity over the latter is practically confined to a distance of a few wavelengths from the edge, and the corresponding part of the integral in (1) is quite unimportant. In this case, the incident waves being still expressed by (8), we can put $-\partial \phi / \partial n=i k C$ with sufficient accuracy over the whole area of the aperture, whence

$$
\phi_{P}=\frac{i k C}{2 \pi} \iint \frac{e^{-i k r}}{r} d S .
$$

For the methods of approximating to the value of this integral, by the use of Huygens' or Fresnel's "zones," or otherwise, we must refer to books on Optics. It is found that the amplitude is nearly uniform within the space bounded by a cylindrical surface whose generators are normal to the screen through the edge of the aperture, and is nearly zero in the surrounding region. Near the cylindrical boundary, on either side, we have

* See Fig. 75, p. 247, which represents the configuration of the equipotential surfaces.

+ The awkwardness of this conclusion may be aroided by giving the screen a certain thickness, and rounding the edges. 
the diffraction effects which are especially studied in the theory of Light.

The question of the impact of waves on a plane lamina can be treated in a similar manner. For this purpose the formula (3) is most convenient. The lamina being in the plane $x=0$, and the primary waves being represented by (8), we may write

$$
\phi=C e^{-i k x}+\chi
$$

where $\chi$ is the velocity-potential due to a vibration of the lamina normal to its plane with the velocity $-i k C$, equal and opposite to that in the primary waves. It is evident that the values of this function at any two points which are symmetrically situated with respect to the plane $x=0$ will be equal in magnitude but opposite in sign. We have then, to the right of the lamina

$$
\chi_{P}=-\frac{1}{2 \pi} \iint \chi \frac{\partial}{\partial x}\left(\frac{e^{-i k r}}{r}\right) d S .
$$

This only requires a knowledge of the value of $\chi$ at the positive face of the lamina, the value at all other points of the plane $x=0$ being obviously zero. The case where the dimensions of the lamina are small compared with $\lambda$ has been noticed in $\S 81$; the scattered waves have then a much smaller intensity than those transmitted by an aperture of the same size and shape. In the opposite extreme, the value of $\chi$ near the positive face is, except near the edge, the same as in the case of an infinite vibrating plate, viz. $\chi=-C e^{i k x}$, so that we have with sufficient accuracy

$$
\chi=\frac{C}{2 \pi} \iint \frac{\partial}{\partial x}\left(\frac{e^{-i k r}}{r}\right) d S .
$$

A detailed study of this integral would indicate, in the complete solution expressed by (11), the existence of a sound-shadow to the right of the lamina. For large values of $\mathrm{kr}$ the formula (13) may be replaced by

$$
\chi=-\frac{i k C}{2 \pi} \iint \frac{e^{-i k r}}{r} \cos \theta d S,
$$

and for small obliquities $\theta$ we may further put $\cos \theta=1$. The formula then becomes, except as to sign, identical with (10), shewing that the disturbance produced by the lamina is, under 
the conditions postulated, exactly opposite to that transmitted by an aperture of the same dimensions. This is a familiar fact in Optics; but the preceding considerations shew that it may be utterly wide of the mark when the wave-length is no longer small compared with the linear dimensions concerned.

It need hardly be said that there are acoustical phenomena where, as in the case of large reflecting or obstructing surfaces, optical relations are approximated to. The results are then analogous, the resemblance being more complete the higher the pitch of the note sounded. By the use of a source of very high pitch, and of a sensitive flame as a detector, Lord Rayleigh has succeeded in imitating some of the most delicate phenomena of physical optics.

In the above theoretical investigation we have been obliged to rely to some extent on intuitive considerations, as e.g. in the assumed distribution of velocity over the area of an aperture when the wave-length is relatively small. It is therefore desirable that such assumptions should be tested if possible by exact calculation. The only instance, at present, where this has been successfully carried out is that of waves incident on a plane screen with a straight edge. The reflection by the screen, the transmission past the edge, the formation of a shadow behind the screen, and the diffraction phenomena near the boundaries of the respective regions, all come out in practical accordance with the usual theory. The investigation was published by Sommerfeld in 1895*.

* A simplified version is given in the Proc. Lond. Math. Soc. (2), vol. Iv. (1906). 


\section{CHAPTER IX}

\section{PIPES AND RESONATORS}

\section{Normal Modes of Rectangular and Spherical} Vessels.

The main object in this chapter is to develop the laws of vibration of air contained in cavities, such as those of resonators and organ pipes, which are in communication with the external atmosphere. A little space may however be devoted in the first instance to some problems relating to the vibrations of air in spaces which are completely enclosed by rigid walls. These will at all events supply some interesting examples of the general theory of normal modes $(\$ 16)$.

The analytical process consists in finding solutions of the equation

$$
\nabla^{2} \phi+k^{2} \phi=0
$$

consistent with the condition

$$
\frac{\partial \phi}{\partial n}=0,
$$

which expresses that the component of the fluid velocity in the direction of the normal $(n)$ vanishes at the boundary. It appears that, as in former analogous problems, this is only possible for a certain sequence of values of $k$, which determine the nature and the frequency of the respective normal modes.

In the case of a rectangular cavity we take the origin at a corner, and the coordinate axes along the edges which meet there. If the lengths of these edges be $a, b, c$, the condition (2) is fulfilled by

$$
\phi=C \cos \frac{p \pi x}{a} \cos \frac{q \pi y}{b} \cos \frac{r \pi z}{c},
$$


where $p, q, r$ are any integers; and the equation (1) is also satisfied provided

$$
k^{2}=\pi^{2}\left(\frac{p^{2}}{a^{2}}+\frac{q^{2}}{b^{2}}+\frac{r^{2}}{c^{2}}\right) .
$$

If we put $q=0, r=0$, the case degenerates into that of the doubly closed pipe $(\$ 62)$.

A more interesting case is that of a spherical cavity. The symmetrical radial vibrations come under the methods of $\$ 71$, 76. The formula (15) of $\$ 71$, which implies that there is no source at the origin, gives, in the case of simple-harmonic vibrations,

$$
\begin{aligned}
& \phi=A \frac{e^{i k r}-e^{-i k r}}{r}, \\
& \phi=C \frac{\sin k r}{r} .
\end{aligned}
$$

or, say,

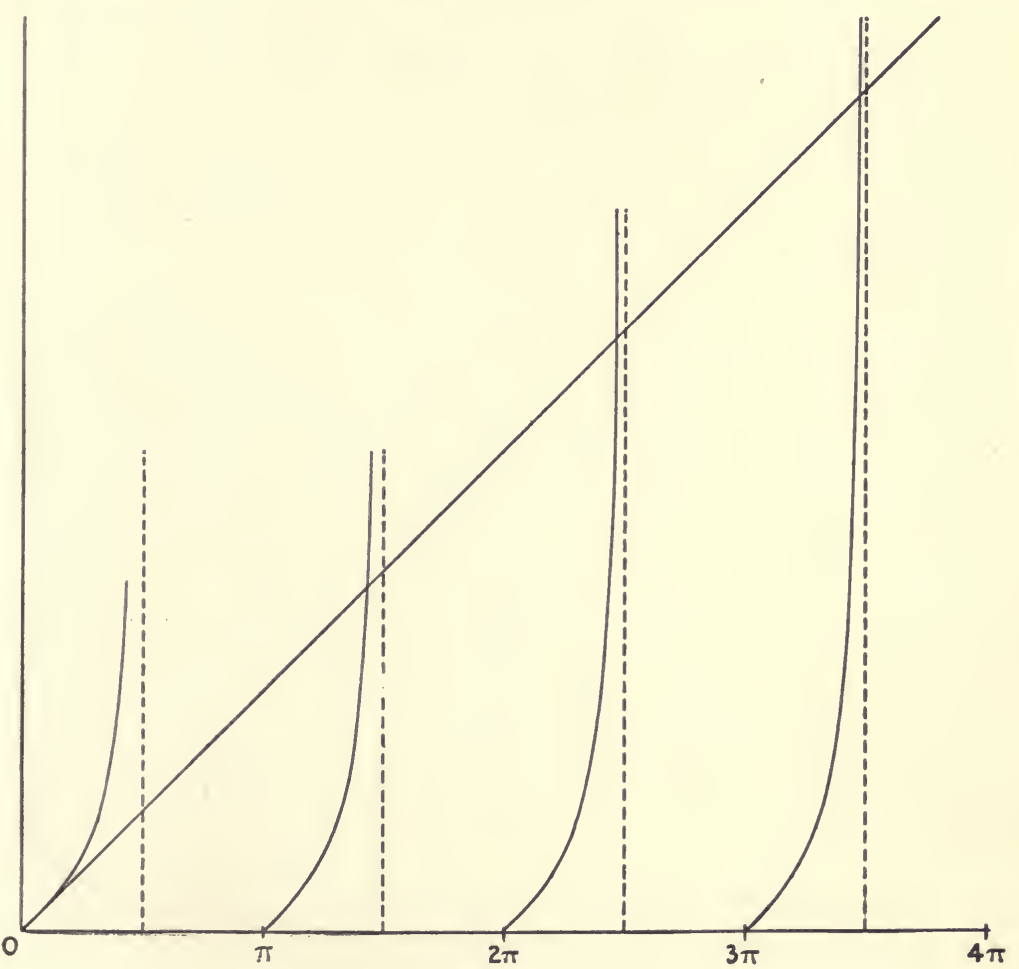

Fig. 76. 
The condition (2) requires that $\partial \phi / \partial r=0$ for $r=\alpha$, the radius of the cavity. Hence

$$
\tan k a=k a .
$$

This is a transcendental equation to find $k$, and thence $n(=k c)$. The roots are obtained graphically (see Fig. 76) as the abscissae of the intersections of the lines $y=\tan x, y=x$, the zero root being of course excepted as irrelevant. We have, approximately, $k a=\left(m+\frac{1}{2}\right) \pi$, where $m=1,2,3, \ldots$ More accurate values of the first three roots are

$$
k a / \pi=1 \cdot 4303,2 \cdot 4590,3 \cdot 4709 \text {. }
$$

The numbers give the ratio of the diameter $2 a$ of the cavity to the wave-length. In the modes after the first there are internal spherical nodes (i.e. surfaces of zero velocity) whose relative positions are indicated by the roots of inferior rank. In the higher modes the nodal surfaces terid, as we should expect, to become equidistant, since the conditions, except near the centre, approximate to those of plane waves.

Equations of somewhat similar structure to (7) occur (as we have seen) in various parts of our subject, as well as in other branches of mathematical physics, and processes of numerical solution have been devised by Euler, Lord Rayleigh and others. There is one method, of very general application, which is so elegant, and at the same time so little known, that it may be worth while to explain it. It is given by Fourier in his Théorie de la Chaleur (1822). Starting with a rough approximation, say $x=x_{1}$, to a particular root of (7), we calculate in succession the quantities $x_{2}, x_{3}, x_{4}, \ldots$ determined by the relations

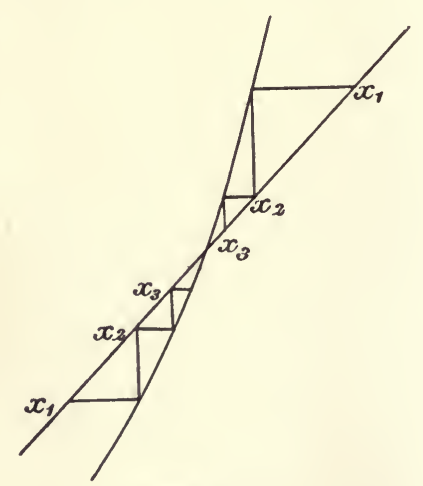

Fig. 77.

$$
x_{2}=\tan ^{-1} x_{1}, x_{3}=\tan ^{-1} x_{2}, x_{4}=\tan ^{-1} x_{3}, \ldots
$$

The figure illustrates the manner in which these converge towards the desired root as a limiting value, no matter from 
which side we start. Some fairly obvious precautions are necessary in using the method, and it is easily seen that the convergence will be slow if the two curves have nearly the same inclination (in the same or in opposite senses) to the axis of $x$. Expressed as multiples of $\pi$, the successive approximations obtained in this way to the first root of (7) are*

$1 \cdot 5,1 \cdot 433435,1 \cdot 430444,1 \cdot 430304,1 \cdot 430297, \ldots$

The same analysis can obviously be applied to the theory of vibrations in a conical pipe whose generating lines meet in 0 . If the tube extend from the origin to $r=a$, the usual approximate condition $(s=0)$ to be satisfied at the open end gives

$$
\sin k a=0,
$$

the same as for a doubly open pipe of length $a(\$ 62)$. For the case of a tube extending from $r=a$ to $r=b$, and open at both ends, we require the complete solution

$$
r \phi=A \cos k r+B \sin k r .
$$

The conditions give

$$
A \cos k a+B \sin k a=0, \quad A \cos k b+B \sin k b=0,
$$

whence

$$
\sin k(b-a)=0,
$$

as in the case of a doubly open pipe of length $b-a$.

If $\chi$ be any solution of the general equation (1), it appears on differentiation throughout with respect to $x$ that the equation is also satisfied by $\phi=\partial \chi / \partial x$. We have already had an example of this in the general double source of $\S 73$. From (6) we derive in this way the solution

or, if $x=r \cos \theta$,

$$
\phi=C \frac{\partial}{\partial x}\left(\frac{\sin k r}{r}\right)
$$

$$
\phi=C \frac{\partial}{\partial r}\left(\frac{\sin k r}{r}\right) \cos \theta=\frac{C}{r^{2}}(k r \cos k r-\sin k r) \cos \theta
$$

This leads to another series of normal modes of the air con-

* In calculations of this kind, and for the purposes of mathematical physics generally, trigonometrical tables based on the centesimal division of the quadrant are most convenient. A four-figure table of this type is included in J. Hoüel's Recueil des Formules et des Tables Numériques, 3rd ed., Paris, 1885. 
tained in a spherical cavity. The condition $\partial \phi / \partial r=0$ is satisfied for $r=a$, provided

$$
\tan k a=\frac{2 k a}{2-k^{2} a^{2}} .
$$

The solution can be carried out as in the case of (7). The annexed diagram of the curves $y=\cot x, y=\left(2-x^{2}\right) / 2 x$, shews that the roots tend after a time to the form $m \pi$. Approximate values of the first few roots are

$$
k a / \pi=\cdot 6625,1 \cdot 891,2 \cdot 930,3 \cdot 948,4 \cdot 959,
$$

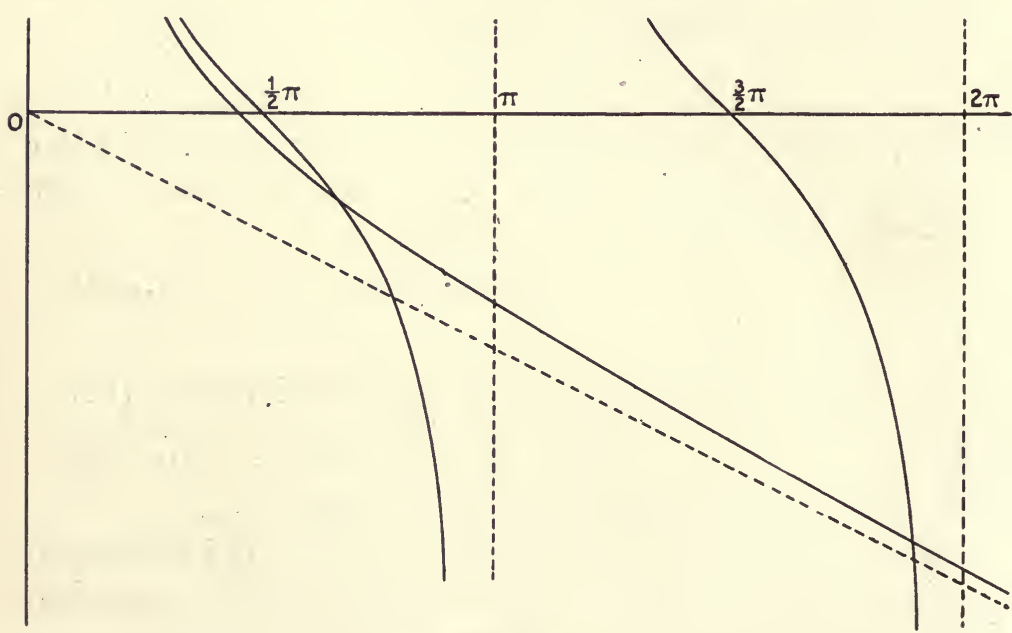

Fig. 78.

the first of which alone gives any trouble. This root corresponds to the gravest of all the normal modes of the cavity. The air swings from side to side, much as in the case of a doubly closed pipe, and the wave-length is $\lambda=2 \pi / k=1.509 \times 2 a$. The forms of the equipotential surfaces, to which the directions of vibration of the air-particles are orthogonal, are shewn in Fig. 79. In the next mode the radial velocity vanishes over the sphere $r / a=\cdot 6625 / 1 \cdot 891=\cdot 350$.

The study of the more complicated normal modes of vibration in a spherical vessel would lead us too far. The problem is fully discussed in Lord Rayleigh's treatise. 


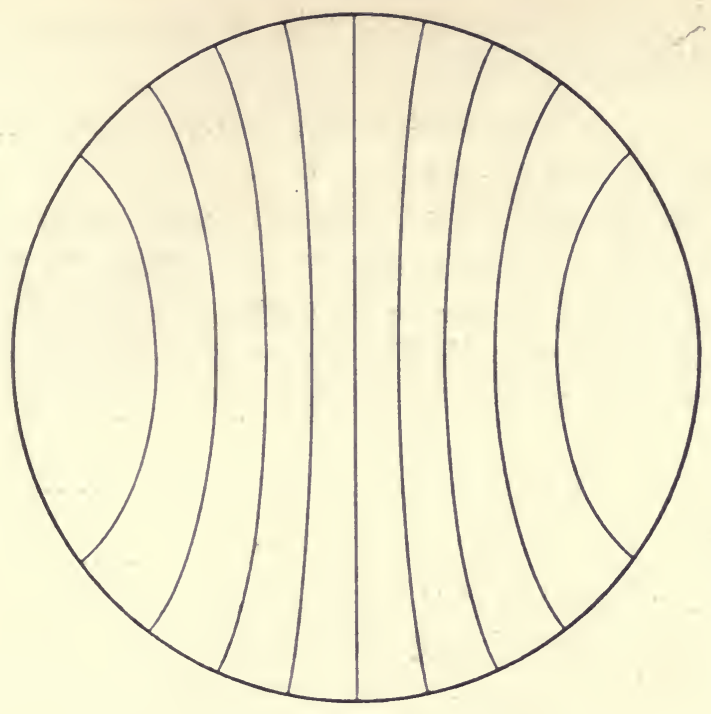

Fig. 79.

\section{Vibrations in a Cylindrical Vessel.}

The theory of the purely transversal vibrations of the air enclosed by a circular cylinder is very similar. As in $\S 54$, the equation

$$
\frac{\partial^{2} \phi}{\partial x^{2}}+\frac{\partial^{2} \phi}{\partial y^{2}}+k^{2} \phi=0,
$$

where $x, y$ are Cartesian coordinates in the plane of a crosssection, becomes in polar coordinates

$$
\frac{\partial^{2} \phi}{\partial r^{2}}+\frac{1}{r} \frac{\partial \phi}{d r}+\frac{1}{r^{2}} \frac{\partial^{2} \phi}{\partial \theta^{2}}+k^{2} \phi=0
$$

and the typical solution, when there is no source at the origin, is

$$
\begin{gathered}
\phi=C J_{m}(k r) \text { cos } \\
J_{m}^{\prime}(k a)=0 .
\end{gathered}
$$

The admissible values of $k$ are determined by the condition that $\partial \phi / \partial r=0$ for $r=a$, or

For the radial vibrations $(m=0)$ the earlier roots are given by

$$
k a / \pi=1 \cdot 2179,2 \cdot 2330,3 \cdot 2383, \ldots,
$$


the limiting form being integer $+\frac{1}{4}$. In the case $m=1$, which includes the gravest mode,

$$
k a / \pi=586,1 \cdot 697,2 \cdot 717, \ldots,
$$

the limiting form being integer $-\frac{1}{4}$.

The purely longitudinal modẹs of a closed circular cylinder come under $\S 62$. There remain the vibrations of mixed type. The equation (2) has now to be modified by the inclusion of a term $\partial^{2} \phi / \partial z^{2}$, where $z$ is the longitudinal coordinate. It is found that the equation is satisfied by

$$
\phi=C \cos \frac{m^{\prime} \pi z}{l} J_{m}(\beta r) \cos m \theta,
$$

provided

$$
k^{2}=\beta^{2}+m^{\prime 2} \pi^{2} / l^{2},
$$

the origin being taken at the centre of one end. The condition of zero normal velocity $(\partial \phi / \partial z)$ at the other end $(z=l)$ is satisfied if $m^{\prime}$ be integral. The corresponding condition at the cylindrical surface requires that $\beta$ should be a root of

$$
J_{m}^{\prime}(\beta a)=0 \text {. }
$$

\section{Free Vibrations of a Resonator. Dissipation.}

The foregoing examples are of theoretical rather than practical interest, since the vibrations of a mass of air enclosed by rigid walls would be completely isolated. For acoustical purposes the vibrating mass must have some communication with the external atmosphere; on the other hand it is essential that the communication should be so restricted that the fraction of the energy which is used up in a single period in the generation of diverging waves shall still be very small. Otherwise the free vibrations could hardly be regarded as approximately simple-harmonic, and might even resemble the "dead-beat". type (§ 11).

The theory is simplest in the case of "resonators" such as were employed by Helmholtz in his researches on the quality of musical notes. These are nearly closed vessels, with an aperture, and are used to intensify, by sympathetic vibration of the enclosed air, the effect of a simple tone produced in the neighbourhood. The precise form is not important; it may be spherical or cylindrical, or almost any shape, so long as 
the least diameter considerably exceeds the dimensions of the aperture. In his synthetic work on the vowel sounds Helmholtz used cylindrical resonators having a circular opening at the centre of one end. When the object was to detect and to isolate a particular overtone in a complex sound, he used the more convenient form shewn in Fig. 80. The small open nipple opposite the mouth is inserted into the ear cavity, so that the tympanic membrane becomes part of the internal wall of the resonator.

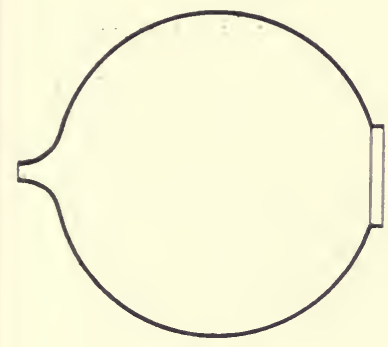

Fig. 80 .

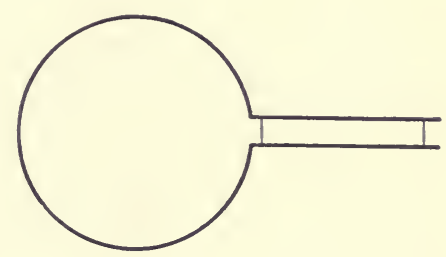

Fig. 81.

The theory of resonators was treated mathematically for the first time by Helmholtz in 1860, and was afterwards greatly simplified by Lord Rayleigh (1871). Suppose in the first place that we have a vessel with a narrow cylindrical neck which is occupied by a plug or piston freely movable to and fro (Fig. 81). Let $Q$ denote the capacity of the vessel, $l$ the length of the neck, $\omega$ its sectional area, $\rho^{\prime}$ the density of the piston. We will assume that the period of vibration is so long that the corresponding wave-length $(\lambda)$ in air is large compared with the diameter of the vessel. Under this condition the condensation $s$ will at any instant be almost uniform throughout the interior, and we may put $s=-\omega x / Q$, where $x$ denotes the small displacement of the piston outwards from its mean position. The resulting excess of pressure on the base of the piston is $\rho c^{2} s \omega$, or $-\rho c^{2} \omega^{2} x / Q$, and the equation of motion of the system is, approximately,

$$
\rho^{\prime} \omega l \ddot{x}=-\rho c^{2} \omega^{2} x / Q .
$$


The motion is accordingly simple-harmonic, with a period $2 \pi / n$, provided

$$
n^{2}=\frac{c^{2} \omega}{l Q} \cdot \frac{\rho}{\rho} .
$$

The nature of the piston is of little importance, provided its mass be sufficiently small. We may even replace it by air, if the length $l$ be small compared with $\lambda$, for under this condition the column of air in the neck will behave almost as if it were incompressible. We have then $\rho^{\prime}=\rho$, and

$$
n^{2}=c^{2} \omega / l Q
$$

Even in the case of a resonator whose mouth consists of a mere opening in the wall, without a neck, the theory is not very different. It is only a question of obtaining a proper measure of the inertia of the mass of air in the immediate neighbourhood of the mouth, inside and outside, which takes the place of the piston in the above problem. The flow through the aperture at any instant is still regulated, approximately, by the same laws as that of an incompressible fluid, or of electricity in a uniform conductor. There being little motion in the interior, the value of $\phi$ there will be sensibly uniform; we denote it by $\phi_{1}$. Outside, at a short distance beyond the mouth, we shall have $\phi=0$, nearly. If $q$ denote the volume of air which has passed through the aperture outwards up to time $t$, the current, or flux, outwards at this instant will

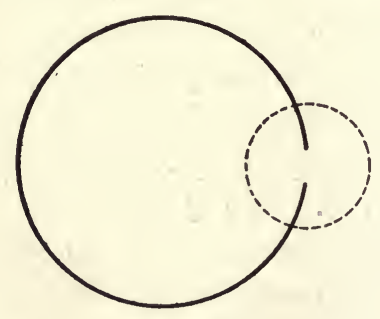

Fig. 82. be $\dot{q}$, and we have, by the electric analogy,

$$
\dot{q}=K \phi_{1},
$$

where $K$ is the "conductivity" ( $\$ 82)$, which depends, of course, on the shape and size of the aperture and the configuration of the wall in its neighbourhood. It is to be observed that this relation (4) is purely kinematical; from the point of view of the generalized dynamics of a system of one degree of freedom 
(§ 7), it expresses the momentum (which may. be symbolized by $\rho \phi_{1}$ ) in terms of the velocity $\dot{q}$. The dynamical equation

$$
c^{2} s=\dot{\phi}_{1}
$$

of $\S 70$ (3) may in like manner be interpreted as expressing the relation between change of momentum and force. If the zero of $q$ correspond to the equilibrium state, we have

$$
s=-q / Q \text {. }
$$

Eliminating $s$ and $\phi_{1}$ between (4), (5), and (6), we obtain

$$
\frac{d^{2} q}{d t^{2}}+\frac{K c^{2}}{Q} q=0 .
$$

The motion is therefore of the type

provided

$$
\begin{gathered}
q=C \cos (n t+\epsilon), \\
n^{2}=K c^{2} / Q .
\end{gathered}
$$

If we write $n=k c$, this gives

$$
k^{2}=K / Q, \quad \lambda=2 \pi \sqrt{ }(Q / K) .
$$

The wave-length depends, as we should expect, solely on the linear dimensions of the resonator and its aperture. For resonators which are geometrically similar in all respects, it varies directly as the linear dimension. This is in accordance with a general principle which may be inferred from the differential equation (2) of $\S 76$, or otherwise. The formula (9) indicates further that the pitch of the resonator is lowered by contracting or partially obstructing the aperture, whilst it is raised by diminishing the internal capacity.

The kinetic energy, being mainly resident in the neighbourhood of the mouth, may be calculated from the principles applicable to an incompressible fluid. If the actual motion were generated instantaneously from rest, the work required would be the sum of half the products of the impulses into the corresponding velocities. The equations (9) of $§ 69$ shew that the requisite impulsive pressure is $\rho \phi_{1}$; hence

$$
T=\frac{1}{2} \rho \phi_{1} \dot{q}=\frac{1}{2}(\rho / K) \cdot \dot{q}^{2} .
$$

The potential energy is, by $\S 70(8)$,

$$
\forall=\frac{1}{2} \rho c^{2} s^{2} Q=\frac{1}{2}\left(\rho c^{2} / Q\right) \cdot q^{2} \text {. }
$$

The coefficients in these expressions being known, the speed $n$ of the oscillations can be inferred at once by the general 
formula (7) of $\S 7$. It was under this form that the theory was presented by Lord Rayleigh. It is to be noticed that the inertia-coefficient is proportional to the "resistance" of the aperture (in the electrical sense), whilst the cuefficient of stability, or elasticity, varies inversely as the capacity $Q$.

The preceding theory applies only to the gravest mode of the resonator. In the higher modes the internal space is divided into compartments by one or more "loop surfaces" (i.e. surfaces of constant pressure, where $\dot{\phi}=0$ ), and the frequencies are much greater. The wave-length is then at most comparable with the linear dimensions, as in the problems of $\S 84$.

As already stated ( $\$ 82)$ the calculation of $K$ is usually difficult. For a circular aperture in a thin wall $K$ is equal to the diameter, and for any form differing not too much from a circle we may put $K=2 \sqrt{ }(\omega / \pi)$, approximately, where $\omega$ is the area. The frequency, as determined by (9), will then vary as $\omega^{\frac{1}{4}} / Q^{\frac{1}{2}}$. It is remarkable that this law was arrived at empirically by Sondhauss at a date (1850) anterior to the theory. When the aperture is fitted with a cylindrical neck, the conductivity is limited mainly by the neck itself, and we may put $K=\omega / l$, approximately, where $l$ is the length. The formula (9) then agrees with (3). It is implied that $l$ is small compared with $\lambda$, and at the same time large compared with the diameter of the channel.

We have in the above theory allowed for the inertia of the external atmosphere, but not for its compressibility, and the vibrations as given by (8) are accordingly persistent. In other words, we have neglected the apparent* dissipation of the energy of the resonator due to air-waves diverging outwards from the neighbourhood of the mouth. This will have, in general, no appreciable influence on the period, but will manifest itself by a gradual decay of the amplitude.

The effect can be estimated with sufficient accuracy indirectly. The flux outwards at the mouth is, by (8),

$$
\dot{q}=-n C \sin (n t+\epsilon) \text {. }
$$

* True dissipative influences such as viscosity and thermal conduction are ignored in the present investigation. They probably play as a rule a wholly subordinate part. 
If the resonator were practically isolated in space, then on account of the assumed smallness of its dimensions as compared with $\lambda$, the effect of the flux at a distance would be that of a simple source of strength $n C$, and the rate of emission of energy would accordingly be

$$
W=n^{4} \rho C^{2} / 8 \pi c,
$$

by the formula (15) of $\S 76$. The energy $E$ of the motion, being equal to the potential energy at its maximum, is, approximately,

$$
E=\frac{1}{2} \rho c^{2} C^{2} / Q
$$

by (12). Equating, on the principles of $\$ 11$, the rate of decay of this energy to the emission $W$, we find

$$
\frac{d C}{d t}+\frac{C}{\tau}=0
$$

and therefore

$$
q=C_{0} e^{-t / \tau} \cos (n t+\epsilon),
$$

provided

$$
\tau=8 \pi c^{3} / n^{4} Q=8 \pi Q / K^{2} c,
$$

in virtue of (9). The ratio of the modulus of decay to the period $(2 \pi / k c)$ is given by

$$
4 k Q / K^{2}=4 Q^{\frac{1}{2}} / K^{\frac{3}{2}}
$$

Since $K$ is at most comparable with the mean breadth of the aperture, this ratio is usually very great, and the preliminary assumptions implied in the above process are amply justified.

If the mouth of the resonator were furnished with an infinite flange, i.e. one whose breadth is large compared with $\lambda$, the equivalent source would, as explained in $\$ 82$, have double the strength above assumed, and the emission of energy, now operative in one half of the surrounding region, would be twice as great. The modulus (18) would accordingly be halved.

As a numerical illustration of the theoretical results, take the case of a spherical vessel $10 \mathrm{~cm}$. in diameter, with a circular aperture $1 \mathrm{~cm}$. in radius, so that $Q=523 \cdot 6, K=2$. The wavelength, calculated from (10), is $101 \cdot 6$; and the frequency therefore about 327. The modulus of decay, as given by (18), is about one-tenth of a second. 


\section{Corrected Theory of the Organ Pipe.}

The same principles can be applied to obtain a correction to the imperfect theory of the open pipe which was given in $\S 62$. We may begin by a brief examination of the slightly simpler problem of reflection at an open end of an infinitely long pipe (\$61).

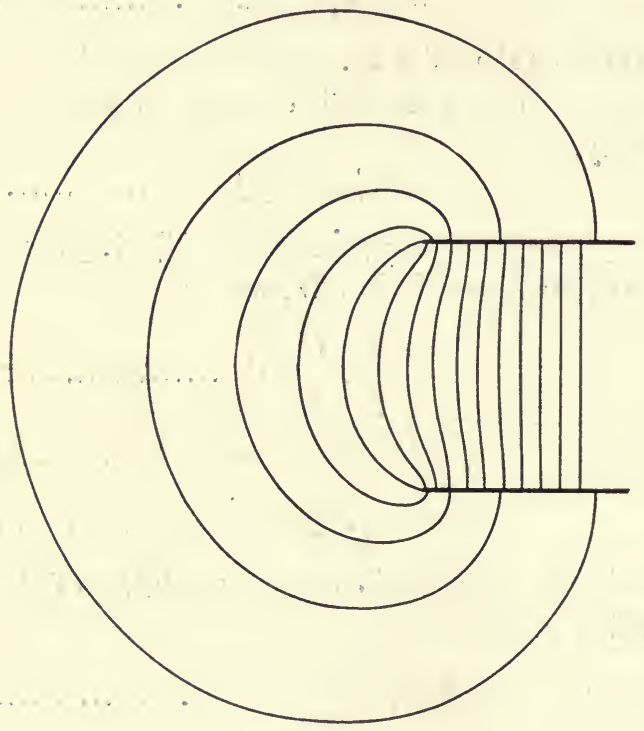

Fig. 83.

Near the open end there is a certain region, whose dimensions are small compared with the wave-length, within which the transition takes place from plane waves within the tube to diverging spherical waves outside*. We take the origin inside the tube, near the mouth, but in the region of plane waves, and the positive direction of the axis of $x$ along the tube. For the region of plane waves we may write

$$
\phi=A e^{i k x}+B e^{-i k x},
$$

where the first term may be taken to represent a train of waves approaching the end, from the right, whilst the second term

* The figure, which is based on formulae given by Helmholtz in another connection, relates to the two-dimensional form of the problem. In three dimensions the transition to a state of uniform radial flow outwards from the mouth would be still more rapid. 
represents the reflected waves. The outward velocity at $O$ is therefore represented by $i k(A-B)$, and the flux is

$$
\dot{q}=i k \omega(A-B) \text {, }
$$

where $\omega$ is the sectional area. The velocity-potential at $O$ is $A+B$. The "resistance" between the section $x=0$ and the external region to the left may be specified as equivalent to that of a certain length $\alpha$ of the pipe, and is accordingly denoted by $\alpha / \omega$. Hence, by the electrical analogy,

$$
A+B=\frac{\alpha}{\omega} \times i k \omega(A-B),
$$

whence

$$
\frac{B}{A}=-\frac{1-i k \alpha}{1+i k \alpha} \text {. }
$$

If we put

$$
k \alpha=\tan k \beta \text {, }
$$

this may be written

$$
B / A=-e^{-2 i k \beta} \text {. }
$$

Hence

$$
\phi=A\left\{e^{i k x}-e^{-i k(x+2 \beta)}\right\} .
$$

The reflected train is therefore equal in amplitude to the incident one, as was to be expected, since the inertia only of the external air is so far taken into account; but there is a difference of phase. In the theory of $\S 61$ the condition to be satisfied at an open end was $s=0$, or $\phi=0$. Hence if we write (7) in the form

$$
\phi=A e^{-i k \beta}\left\{e^{i k(x+\beta)}-e^{-i k(x+\beta)}\right\}
$$

we recognize that the circumstances are the same as if the pipe were prolonged to the left for a length $\beta$, and the reflection at the mouth were to take place according to the rudimentary theory. The wave-length being assumed to be large compared with the diameter of the pipe, $k \alpha$ will usually be small, so that $\beta=\alpha$, nearly. But if the pipe be very much contracted or obstructed at the mouth, $k \alpha$ may be considerable, and $k \beta$ will in that case approach $\frac{1}{2} \pi$. We then have $B=A$, nearly, and the circumstances approximate to those of reflection at a closed end.

The actual determination of $\alpha$ is a problem in electric conduction which has at present only been solved, even approximately, in a very few cases. Lord Rayleigh estimates that for an accurately cylindrical tube fitted with an infinite flange the value of $\alpha$ is about 82 of the sectional radius. For 
an unflanged cylindrical tube experiment seems to indicate a value of about 6 of the radius.

We will next suppose the pipe to be of finite length, and to be closed at $x=l$, the origin being chosen as before, near the mouth, in the region of plane waves. For this latter region we may assume

$$
\phi=A \cos k(l-x),
$$

since $\partial \phi / \partial x$ must vanish for $x=l$. The flux outwards at the mouth is therefore

$$
\dot{q}=\omega \partial \phi / \partial x=k \omega A \sin k l,
$$

and the potential at $O$ is $A \cos k l$. Hence with the same meaning of $\alpha$ as before we have

$$
A \cos k l=\frac{\alpha}{\omega} \times k \omega A \sin k l,
$$

or

$$
\cot k l=k \alpha \text {. }
$$

This equation determines the wave-lengths $(2 \pi / k)$ of the various normal modes. Usually, $k \alpha$ is small, and the solution of (11) is then

or

$$
\begin{gathered}
k l=\left(m+\frac{1}{2}\right) \pi-k \alpha, \\
k(l+\alpha)=\left(m+\frac{1}{2}\right) \pi,
\end{gathered}
$$

where $m$ is integral. The character of the normal modes is therefore the same as on the rudimentary theory ( $\$ 62)$, provided we imagine the length of the pipe to be increased by the quantity a. In particular, the frequencies are as the odd integers $1,3,5, \ldots$, so long as the wave-length remains large compared with the diameter.

If the aperture be contracted the value of $\alpha$ is increased, and the result tends to become less simple. In particular, the harmonic relation of the successive frequencies is violated, as may easily be seen from a graphical discussion of the equation (11). When the pipe is almost closed, $\alpha$ is relatively great, and the solution of (11) is $k l=1 / k \alpha$, or $k^{2}=1 / l \alpha$. This agrees with the formula (10) of $\S 86$, if we put $\omega l=Q, \omega / \alpha=K$.

In the case of a pipe open at both ends the period equation is found to be

$$
\tan k l=-k\left(\alpha+\alpha^{\prime}\right)
$$

where $\alpha, \alpha^{\prime}$ are the corrections for the two ends, but the calcula- 
tion implies that $k \alpha$ and $k \alpha^{\prime}$ are small. It is, however, only on this condition that the conductivities at the two ends can, as a rule, be estimated independently of one another. The equation is then equivalent to

$$
\sin k\left(l+\alpha+\alpha^{\prime}\right)=0,
$$

and the frequencies are therefore those which are assigned to a pipe of length $l+\alpha+\alpha^{\prime}$ by the rudimentary theory. The harmonic relation between the various normal modes is preserved, but it must be remembered that the approximation is the more precarious, the higher the order of the harmonic.

The wave-lengths of the proper tones are in all cases fixed by the linear dimensions, but the frequencies, which vary as the velocity of sound, will rise or fall with the temperature. An "open" organ pipe is tuned by means of a contrivance which increases or diminishes the effective aperture at the open end, i.e. the end remote from the "mouth" proper. The pitch of a "closed" pipe is regulated by adjusting the position of a plug which forms the barrier.

To calculate the rate of decay of the free vibrations it will be sufficient to take the case of the stopped pipe. The kinetic energy corresponding to

$$
\phi=A \cos k(l-x) \cos n t
$$

is given with sufficient accuracy by

$$
T=\frac{1}{2} \rho \omega \int_{0}^{l}\left(\frac{\partial \phi}{\partial x}\right)^{2} d x=\frac{1}{4} \rho k^{2} \omega l \cdot A^{2} \cos ^{2} n t,
$$

if $k \alpha$ be small, since $\cos k l=0$, nearly. A more careful calculation, taking account of the transition region between the plane and the spherical waves, replaces $l$ by $l+\alpha$, approximately, in this formula, but the correction is not important. The total energy, being equal to the kinetic energy at its maximum, is accordingly

$$
E=\frac{1}{4} \rho k^{2} \omega l A^{2} \text {. }
$$

If the mouth be unflanged it acts, in relation to the external space, as a simple source of strength $k \omega A \sin k l$, or $k \omega A$, nearly, and the consequent emission of energy per second is accordingly

$$
W=\rho k^{\star} \omega^{2} c A^{2} / 8 \pi,
$$


by $\S 76(15)$. Equating the rate of decay of the energy to $W$, we are led to the equation

$$
\frac{d A}{d t}+\frac{k^{2} \omega c}{4 \pi l} A=0,
$$

and the modulus of decay is therefore

$$
\tau=4 \pi l / k^{2} \omega c \text {. }
$$

The ratio of this to the period $(2 \pi / k c)$ is $2 l / k \omega$, or (in the gravest mode) $4 l^{2} / \pi \omega$, nearly. Since the moduli of the various normal modes are proportional to the squares of the respective wave-lengths, the decay is the more rapid the higher the order. For a flanged pipe the result (20) would be halved.

\section{Resonator under Influence of External Source.} Reaction on the Source.

The theory of forced vibrations due to an external source of sound, to which we now proceed, involves some rather delicate considerations, and is often misunderstood. That the mass of air contained in a resonator or an organ pipe should be set into vigorous vibration by a source in approximate unison with it is intelligible enough; but it is further desirable to have some estimate of the amplitude of the forced vibration, and in particular to understand why the sound which is apparently emitted by the resonator should under certain conditions enormously exceed that which would be produced by the original source alone.

For simplicity we will suppose that this source is maintained at constant amplitude by a suitable supply of energy, so that the vibration of the air is everywhere steady. It is evident at once that under this condition no work is done, on the average of a whole period, at the mouth of a resonator on the contained air, the energy of the latter being constant, and consequently that no work can in turn be done by the reaction of this mass on the external atmosphere. Any increased propagation of sound to a distance must be due to the changed conditions which the action of the resonator has introduced in the neighbourhood of the original source. If this source be not maintained constant, but merely started with an initial fund of energy (as in the case of a tuning 
fork), this fund will under the influence of the resonator be more rapidly consumed.

In order to treat the question in a form free from unessential details, which may vary from one case to another, we take the case of a resonator of the type considered in $\S 86$, whose dimensions are small compared with the wave-length.

The theory is simplest when the frequency of the source is very nearly equal to the natural frequency of the resonator, as determined by $\S 86(9)$, so that the forced vibration in the latter is at its strongest. It will perhaps make the matter clearer if we imagine in the first instance that the resonator has a short cylindrical neck in which a thin massless disk, almost exactly fitting it, can be made to move to and fro by a suitable application of force. Suppose then that the disk is made to execute a vibration such that the volume swept over by it outwards up to time $t$ is

$$
q=C \cos n t
$$

and let the extraneous force which must be applied to the disk to compensate the difference of the air-pressures on the two sides be denoted by

$$
A \cos n t+B \sin n t
$$

this expression being (say) positive when the force is outwards. The component $A \cos n t$ which keeps step with the displacement is required to control the inertia of the air. From the general theory of forced vibrations $(\$ 9,12)$ it appears that the coefficient $A$ can be made to have one sign or the other by adjusting the value of $n$, the sign being the same as that of $C$ when the imposed vibration is relatively slow, and the opposite when it is relatively rapid. We may therefore suppose $n$ to be so adjusted that $A=0$. The circumstances are then very nearly those of a free vibration, and the required value of $n$ is given by

$$
n^{2}=K c^{2} / Q,
$$

very approximately. The second component of the force (2), which keeps step with the velocity $(\dot{q})$, is required to maintain the emission of energy outwards, which is, by $§ 76$ (15),

$$
W=n^{4} \rho C^{2} / 8 \pi c \text {. }
$$


This must be equal to the mean value of $p \dot{q}$, where $p$ is the pressure at the outer face of the disk. Hence by comparison we find that $p$ must have the form

$$
p=p_{0}+D \cos n t-\frac{n^{3} \rho C}{4 \pi c} \sin n t .
$$

The corresponding pressure on the inner face will be

$$
p=p_{0}+D \cos n t,
$$

simply, since no work is done, on the whole, on the air contained in the resonator.

We may now invoke the action of the external source. If this be such as would produce the pressure

$$
p=p_{0}+\frac{n^{3} \rho C}{4 \pi c} \sin n t
$$

at the mouth of the resonator if the disk were at rest, then in the motion which is compounded of that due to the source and that due to the disk no extraneous force will be required, and the disk may therefore be annihilated without causing any appreciable change in the conditions. If $\phi_{2}$ be the velocity-potential due to the source alone, at the mouth of the resonator, we must have, in this case,

$$
\phi_{2}=-\frac{n^{2} C}{4 \pi c} \cos n t,
$$

since (7) must be identical with $p=p_{0}+\rho \dot{\phi}_{2}$.

The hypothesis of a rigid disk vibrating in a cylindrical space was merely introduced for facility of conception, and is in no way essential to the argument. The disk may, if we please, be replaced by a flexible and extensible membrane enclosing the aperture of the resonator, and abutting on the external wall in the region of diverging waves.

Comparing (1) with (8) we see that to a disturbing potential whose value at the mouth is

$$
\dot{\phi}_{2}=J \cos (n t-\epsilon)
$$

will correspond a vibration

$$
q=-\frac{4 \pi c}{n^{2}} J \cos (n t-\epsilon)
$$


under the condition of maximum resonance, when $n$ is given by (3) approximately. The corresponding flux is

$$
\dot{q}=\frac{4 \pi J}{k} \sin (n t-\epsilon) \text {. }
$$

The emission of energy is best calculated from a consideration of the circumstances at a great distance. The velocity-potential will be compounded of that due to the original source and that due to the flux $\dot{q}$, and under certain conditions the latter component may greatly preponderate. The emission of energy is then

$$
W=2 \pi \rho c J^{2},
$$

approximately, by $§ 76(15)$.

Thus if $\phi_{2}$ be due to a simple source $A \cos k c t$ at a distance $b$ from the aperture, we have

$$
\phi_{2}=\frac{A}{4 \pi b} \cos k(c t-b) .
$$

Hence

and

$$
\begin{gathered}
J=A / 4 \pi b, \\
\dot{q}=\frac{A}{k b} \sin k(c t-b) .
\end{gathered}
$$

This is equivalent to a source whose amplitude is to that of the primary source in the ratio $1 / k b$. If $b$ be small compared with $\lambda / 2 \pi$ this ratio is large; and the emission of energy exceeds that due to the original source in the ratio $1 / k^{2} b^{2}$.

In the case of a double source $B \cos k c t$ we may write, if $k b$ be small,

$$
\phi_{2}=\frac{B}{4 \pi b^{2}} \cos \alpha \cos k(c t-b)
$$

by $\S 76$ (23), if $\alpha$ denote the angle which the axis of the source makes with the line drawn from it to the aperture. Hence $J=B \cos \alpha / 4 \pi b^{2}$, and the emission, as given by (12), is

$$
W=\rho c B^{2} \cos ^{2} \alpha / 8 \pi b^{4} .
$$

The emission due to the original (double) source alone would be $\rho k^{4} c B^{2} / 24 \pi$, by $\S 76(26)$. The ratio in which the emission is increased is therefore $3 \cos ^{2} \alpha / k^{4} b^{4}$. Since the mean value of $\cos ^{2} \alpha$ is $\frac{1}{3}$, the mean value of this ratio, for all directions of the axis of the double source, is $1 / k^{4} b^{4}$. That the ratio 
should, under the given conditions, be so much greater than in the preceding case is due to the relatively smaller efficiency of a double source, as compared with a simple one, in propagating energy outwards $(\$ 80)$.

It may be well to insist again that the increased output of energy is an indirect consequence of the presence of the resonator, which itself does no work. The whole energy is supplied by the original source, where the motion takes place against an augmented component of pressure in the same phase with the velocity. The velocity-potential due to the flux $\dot{q}$ outwards from the resonator, as given by (11), is

$$
\phi_{1}=\frac{J}{k r} \sin (n t-k r-\epsilon),
$$

and the resultant pressure is

$$
p=p_{0}+\frac{\rho c J}{r} \cos (n t-k r-\epsilon) .
$$

In the case of a simple primary source we had $J=A / 4 \pi b$, $\epsilon=k b$; hence, putting $r=b$, we find that the consequent pressure in the neighbourhood of this source is

$$
p=p_{0}+\frac{\rho c A}{4 \pi b^{2}} \cos (n t-2 k b) \text {. }
$$

Since the imposed outward flux is $A \cos n t$, the mean rate of work against this part of the pressure is

$$
\frac{\rho c A^{2}}{8 \pi b^{2}} \cos 2 k b \text {. }
$$

The output is therefore greater than it would be in the absence of the resonator, in the ratio $\cos 2 k b / k^{2} b^{2}$. This agrees with the former result, obtained on the hypothesis that $k b$ is small.

The energy stored in the resonator under the conditions of maximum vibration is, by $\S 86$ (15),

$$
E=8 \pi^{2} \rho c^{4} J^{2} / n^{4} Q=8 \pi^{2} \rho Q J^{2} / K^{2} .
$$

This varies directly as the capacity $Q$, and is for apertures of similar form inversely proportional to the area.

The effect of a resonator under the influence of a distant 
source in unison with it may be sufficiently illustrated on the assumption that the incident waves are plane. If

$$
\phi_{2}=J \cos k(c t-x) \text {, }
$$

the ratio of the energy scattered by the resonator, which is given by (12), to the energy-flux in the primary waves, viz. $\frac{1}{2} \rho k^{2} c J^{2}$, is $4 \pi / k^{2}$, or $\lambda^{2} / \pi$. The energy diverted per second, at its maximum, is therefore equal to 318 of that which in the primary waves is transmitted across a square area whose side is the wave-length. It may be added that a similar law is met with in the theory of selective absorption of light.

When approximate agreement between the frequency $(n / 2 \pi)$ of the source and the natural frequency $\left(n_{0} / 2 \pi\right)$ of the resonator is no longer assumed, the external pressure which is required to maintain a steady vibration (1) through the aperture will consist of two parts. In the first place we have a component keeping step with the displacement, which is required in order to control the inertia of the air. This is easily found by an extension of the method of $\S 86$. If $\phi_{1}$ denote the velocitypotential in the interior of the resonator, $\phi_{2}$ that at a short distance outside the aperture, in the region of approximately spherical waves, we have

$$
\dot{q}=K\left(\phi_{1}-\phi_{2}\right)
$$

in accordance with the electrical analogy. In the interior we have $s=-q / Q, c^{2} s=\dot{\phi}_{1}$, as before. Hence

$$
\begin{gathered}
\ddot{q}+n_{0}^{2} q=-K \dot{\phi}_{2}, \\
n_{0}{ }^{2}=K c^{2} / Q .
\end{gathered}
$$

This gives, for the external pressure,

$$
p-p_{0}=\rho \dot{\phi}_{2}=\frac{n^{2}-n_{0}^{2}}{K} \rho C \cos n t .
$$

The second part, which is in the same phase as $\dot{q}$, is needed in order that there may on the average be no gain or loss of energy to the air contained in the resonator, and is accordingly given by (7). Hence we have, altogether,

$$
p=p_{0}+\rho C\left(\frac{n^{2}-n_{0}^{2}}{K} \cos n t+\frac{n^{3}}{4 \pi c} \sin n t\right)
$$


and the complete expression for the disturbing velocity-potential near the mouth must be

$$
\phi_{2}=\frac{n C}{K}\left\{\left(1-\frac{n_{0}{ }^{2}}{n^{2}}\right) \sin n t-\frac{k K}{4 \pi} \cos n t\right\} .
$$

In the problem as it actually presents itself the value of $\phi_{2}$ at the mouth is prescribed, say

$$
\phi_{2}=J \cos (n t-\epsilon) ;
$$

and in order to identify this with (28) we must have

Hence

$$
\begin{gathered}
J \cos \epsilon=-\frac{k K}{4 \pi} \cdot \frac{n C}{K}, \quad J \sin \epsilon=\left(1-\frac{n_{0}{ }^{2}}{n^{2}}\right) \frac{n C}{K} . \\
\frac{J^{2}}{n^{2} C^{2}}=\frac{1}{K^{2}}\left\{\left(1-\frac{n_{0}^{2}}{n^{2}}\right)^{2}+\frac{k^{2} K^{2}}{16 \pi^{2}}\right\} . \quad \ldots \ldots .
\end{gathered}
$$

This determines $C$ in terms of $J$. If $\tau$ denote the modulus of decay of free vibrations, as given by $\S 86$ (18), the formula may also be written

$$
\frac{J^{2}}{n^{2} C^{2}}=\frac{1}{K^{2}}\left\{\left(1-\frac{n_{0}^{2}}{n^{2}}\right)^{2}+\frac{4}{n_{0}^{2} \tau^{2}} \cdot \frac{n^{2}}{n_{0}^{2}}\right\} .
$$

Except in the case of approximate synchronism the second term within the brackets will be small compared with the first. Hence for a given value of $J$, the value of $n C$ (which is the amplitude of the flux $\dot{q}$ ) will be greatest when $n=n_{0}$, approximately. Moreover, for a given deviation of the ratio $n / n_{0}$ from unity the intensity of the resonance falls short of the maximum in a greater proportion the greater the value of $n_{0} \tau$, i.e. the greater the ratio of the modulus of decay to the free period. In other words, the smaller the damping of free vibrations, the more sharply defined is the pitch of maximum resonance. This is in accordance with the general theory of $\S 13$.

The vibrations of a resonator under the influence of an internal source of sound are discussed in $§ 90$ with special reference to the theory of reed-pipes.

89. Mode of Action of an Organ Pipe. Vibrations caused by Heat.

Although the loss of energy in a single period may be small, the free vibrations of the column of air contained in an organ 
pipe are practically dissipated in a fraction of a second ; this is owing to the small inertia as compared with that of a pianowire. For musical purposes some device for sustaining the note is required. In the ordinary "flute pipe," the lower part of which is shewn in section in Fig. 84, a thin stream of air is driven by pressure from a wind-chest so as to strike against the bevelled lip of the aperture. Under these circumstances a very slight cause will make the jet pass either wholly inside or wholly outside the pipe. The precise mode of action is obscure, but there can hardly be any doubt that in its main features it is analogous to that of a clock-escapement. Periodic impulses are given by the jet, alternately inwards and outwards, to the air near the mouth, always in the direction in which the air is tending; whilst the vibrating column itself

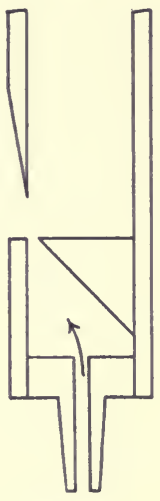

Fig. 84. mainly determines the epochs at which these impulses shall occur. The circumstances are accurately periodic, so that the driving force can be resolved by Fourier's theorem into a series of harmonic components whose frequencies are as $1,2,3, \ldots$ The relative amplitudes with which these are reproduced in the vibrating column will depend on the closeness of their frequencies to the natural frequencies. Thus in a "closed" pipe, i.e. one closed at the upper end, the harmonics of odd order are alone excited. Again the theory of $\S 87$ indicates that in a sufficiently wide pipe the natural frequencies may deviate sensibly from the harmonic relation, in which case only the lower harmonics (after the fundamental) will be sensible; in particular, a wide closed pipe gives almost a pure tone. On the other hand a pipe which is narrow in comparison with the length may give a note rich in harmonics. Indeed, if such a pipe be blown with sufficient force, the fundamental is not sounded at all, the period becoming that of the first harmonic; if the strength of the blast be further increased the note may jump to the next member of the series, and so on. An explanation is probably to be found in the sort of dynamical elasticity possessed by the jet. 
Metal pipes are richer in harmonics than wooden pipes of the same dimensions. This may be partly due to the greater fineness of the lip, which introduces a greater degree of abruptness in the action of the jet, and so favours the amplitude of the terms of higher order in the Fourier series which expresses the driving force. Another source of the contrast in quality may be found in the smaller rigidity and imperfect elasticity of the walls of a wooden pipe, which may tend to absorb the energy, especially in the case of the higher harmonics.

The "speaking" of a resonator of any kind, when a jet of air is blown across its mouth, is to be explained on the same principles. In a resonator of the usual type the normal modes after the first are far removed in pitch from the fundamental, and are not sensibly excited by the essentially periodic impulse. The note obtained is therefore a pure tone.

The vibrations of a column of air may also be excited by the periodic application of heat, as in the well-known experiment of the "singing flame," where a jet of hydrogen burns within an open cylindrical pipe. For the maintenance of the vibration it is necessary that heat should be supplied at a moment of condensation, or abstracted at a moment of rarefaction. To explain how the adjustment is effected, it would be necessary to take account of the fact that the vibrating system includes the gas contained in the supply tube of the jet, as well as the column of air in the pipe. The matter is thus somewhat intricate, but a satisfactory theory has been made out, which accounts clearly for the several conditions under which the experiment is found to succeed or to fail*.

\section{Theory of Reed-Pipes.}

The mechanism of the reed stops of the organ is quite different. The current of air issuing from the wind-chest is made intermittent by its passage through a rectangular aperture in a metal plate, which is periodically opened and closed (partially) by a vibrating metal tongue, or " reed." The period is accordingly determined mainly by the elasticity and inertia of the tongue itself. The vibrations of the latter were 
found by Helmholtz, by direct observation, to be of the simpleharmonic type, but the fluctuations in the current of air are necessarily of a more complex character. If the periodic current be expressed by a Fourier series

$$
C_{0}+C_{1} \cos \left(n t+\epsilon_{1}\right)+C_{2} \cos \left(2 n t+\epsilon_{2}\right)+\ldots, \quad \ldots(1)
$$

the coefficients $C_{2}, C_{3}, \ldots$ are usually by no means insensible as compared with $C_{1}$, and accordingly if the sound is heard directly it has a very harsh and nasal character. In practice, the reed is fitted with a suitable resonator, or "sound-pipe," which specially reinforces one or more of the lower elements in the harmonic series (1).

For the purposes of mathematical treatment we may idealize the question somewhat, and imagine that at a given point in the interior of the resonator we have a simple source of the type corresponding to one of the terms in (1). It appears from the elementary theory of $\S 62$ that in the case of a cylindrical pipe, with the source at one end, the frequencies of maximum resonance are very approximately those of the free vibrations when that end is closed. Hence a reed fitted with a cylindrical sound-pipe of suitable length will emit a series of tones whose frequencies are proportional to the odd integers $1,3,5, \ldots$. In a conical pipe, on the other hand, with the source near the vertex, we have the complete series of harmonics with frequencies proportional to $1,2,3,4, \ldots$ (see $\S 84)$. But in either case the harmonics of high order are discouraged by the increasing deviation of the frequencies of maximum resonance from the harmonic relation which necessarily holds in the expression for the essentially periodic current of air.

As the question is instructive in various ways it may be worth while to examine more in detail the case of a cylindrical sound-pipe (of any form of section), applying the correction for the open end, and allowing for the dissipation due to the escape of sound outwards. The plan of the investigation is similar to that of $\S 87$, the difference being that we now have a source $C e^{i n t}$ (say) at the end $x=l$. For simplicity we will 
assume this source to be distributed uniformly over the crosssection, so that

$$
\frac{\partial \phi}{\partial x}=\frac{C}{\omega} e^{i n t} \quad[x=l] .
$$

Let us suppose for a moment that we have a flux

$$
\dot{q}=A \cos n t
$$

outwards from the mouth. The pressure at $O$ will consist of two components. We have first the part necessary to control the inertia of the air near the mouth; the corresponding part of the velocity-potential just inside is

$$
\phi=\frac{\alpha}{\omega} \dot{q}=\frac{\alpha A}{\omega} \cos n t,
$$

where $\alpha$ has the same meaning as in $\S 87$. Next we have the part which is effective in generating diverging waves outside. On the principles of $\S 88$ this is found to be

$$
\frac{\rho k^{2} c}{4 \pi} A \cos n t
$$

corresponding to

$$
\phi=\frac{k A}{4 \pi} \sin n t
$$

since $k=n / c$. The total velocity-potential at 0 , corresponding to (3), is therefore

$$
\phi_{0}=\left(\frac{\alpha}{\omega} \cos n t+\frac{k}{4 \pi} \sin n t\right) A .
$$

Generalizing this, we may say that to a flux

$$
\dot{q}=A e^{i n t}
$$

corresponds

$$
\phi_{0}=\left(a-\frac{i k \omega}{4 \pi}\right) \frac{A}{\omega} e^{i n t},
$$

the expression (7) being in fact the real part of (9), when $A$ is real. The correspondence will hold even if $A$ be complex, since this is merely equivalent to a change in the origin of $t$.

We now assume, for the region of plane waves,

$$
\phi=\frac{1}{k \omega}\{B \cos k(l-x)-C \sin k(l-x)\} e^{i n t},
$$


where the constants have been adjusted so as to satisfy (2). Comparing with (8) and (9) we find

Hence

$$
\left.\begin{array}{l}
B \sin k l+C \cos k l=A, \\
B \cos k l-C \sin k l=\left(k \alpha-\frac{i k^{2} \omega}{4 \pi}\right) A .
\end{array}\right\}
$$

$$
\left.\begin{array}{l}
B=A\left\{\sin k l+\left(k \alpha-\frac{i k^{2} \omega}{4 \pi}\right) \cos k l\right\}, \\
C=A\left\{\cos k l-\left(k \alpha-\frac{i k^{2} \omega}{4 \pi}\right) \sin k l\right\} .
\end{array}\right\}
$$

The latter equation gives $A$ in terms of $C$. Considering only absolute values we have

$$
\left|\frac{C^{2}}{A^{2}}\right|=(\cos k l-k \alpha \sin k l)^{2}+\left(\frac{k^{2} \omega}{4 \pi}\right)^{2} \sin ^{2} k l .
$$

Since $k^{2} \omega$ is usually a small fraction, the emission of energy, which varies as $\left|A^{2}\right|$, will be greatest for a given source $C \cos n t$ when

$$
\cos k l=k \alpha \sin k l,
$$

nearly, i.e. when the imposed frequency approximates to that of one of the normal modes of the pipe when closed at $x=l$, as determined by $\S 87$ (11). In the case of the reed-pipe, therefore, the tones which are specially reinforced consist of the fundamental and the harmonics of odd order.

When (14) is satisfied, we have by (12)

$$
\frac{C}{A}=\frac{i k^{2} \omega}{4 \pi} \sin k l .
$$

This determines the relation between the flux outwards at the mouth, and that constituting the source. The former now greatly exceeds the latter in amplitude, and the factor $i$ shews that it differs in phase by a quarter-period.

Again, from (10) and (11) we have

$$
\phi=\frac{A}{k \omega}\left\{\sin k x+k \alpha \cos k x-\frac{i k^{2} \omega}{4 \pi} \cos k x\right\} e^{i n t} .
$$


When (14) holds, this reduces to

$$
\begin{aligned}
\phi & =\frac{A}{k \omega \sin k l}\left\{\cos k(l-x)-\frac{i k^{2} \omega}{4 \pi} \sin k l \cos k x\right\} e^{i n t} \\
& =-\frac{4 \pi i C}{k^{3} \omega^{2} \sin ^{2} k l}\left\{\cos k(l-x)-\frac{i k^{2} \omega}{4 \pi} \sin k l \cos k x\right\} e^{i n t} .
\end{aligned}
$$

The real part gives

$$
\phi=\frac{4 \pi C^{\circ}}{k^{3} \omega^{2} \sin ^{2} k l}\left\{\cos k(l-x) \sin n t-\frac{k^{2} \omega}{4 \pi} \sin k l \cos k x \cos n t\right\},
$$

corresponding to a source $C \cos n t$. The variable part of the pressure at the source $(x=l)$ is

$p-p_{0}=\rho \dot{\phi}=\frac{4 \pi \rho c C}{k^{2} \omega^{2} \sin ^{2} k l}\left(\cos n t+\frac{k^{2} \omega}{4 \pi} \sin k l \cos k l \sin n t\right)$.

The first part of this is by far the more considerable; it is, moreover, the only part which is effective in doing work. The mean rate of work done at the source, i.e. the mean value of $p C \cos n t$, is

$$
W=\frac{2 \pi \rho c C^{2}}{k^{2} \omega^{2} \sin ^{2} k l}
$$

It may easily be verified that this is equal to the work spent in generating waves at the mouth, where, by (15),

$$
\dot{q}=\frac{4 \pi C}{k^{2} \omega \sin k l} \sin n t .
$$

It appears further from (19) that the maximum of pressure at $x=l$ synchronises almost with the maximum influx of air, following it however by a short interval. There is therefore a tendency slightly to lower the pitch of the reed, which is, in the instruments here referred to, of the "inbeating type," i.e. the passage is opened when the reed swings inwards, towards the wind-chest. The fact that the resultant force on the reed is approximately in the same phase with the displacement indicates that the reed is vibrating with a frequency somewhat less than that natural to it (\$12). 
The reed-stops of an organ fall in pitch as the temperature rises, owing to the diminished elasticity of the metal tongues; this is the opposite of what happens with regard to the flutepipes ( $\$ 62)$. A reed-pipe is tuned by a contrivance which alters the effective length of the vibrating tongue.

It should be mentioned that there is another class of instruments in which the "reed" has a much smaller elasticity and is mainly controlled by the reaction of the resonant chamber, its own natural frequency being relatively low. The reed is then of the "out-beating type," the aperture being widest when the reed swings outwards, i.e. with the wind. The human larynx comes essentially under this class. 


\section{CHAPTER $\mathrm{X}$}

\section{PHYSIOLOGICAL ACOUSTICS}

\section{Analysis of Sound Sensations. Musical Notes.}

The vibrations of elastic bodies and the propagation of waves through the atmosphere are subject to well-ascertained mechanical laws, and the inferences drawn from these can be controlled by more or less decisive experiments. But when we approach the field where the human mechanism comes into play, we are met by the peculiar difficulties which are inherent in the observation and study of subjective phenomena. In particular, when we endeavour to analyse a familiar complex sensation into its elements, we are attempting a task for which the experience of daily life has peculiarly unfitted us. Thus we may have been accustomed to interpret the sensation in question as indicating the presence of a particular object, or the occurrence of a particular kind of event, in a particular place. The elements of which it is made up give individually little or no information; it is the combination which is significant, and attention to the details would only distract from what is of immediate practical interest. To use a rough and indeed an utterly inadequate illustration, it is as if we were to insist upon spelling every word we read.

The theory of sense-perception, especially in relation to optics and acoustics, is a fascinating subject, but it cannot be dealt with here. The student who is unversed in it may be referred to the writings of Helmholtz*.

* The theory is explained in its acoustical bearings in the Tonempfindungen, already cited (p. 3). It is also discussed from the optical point of view in his Handbuch der physiologischen Optik, 2nd ed., Hamburg and Leipzig, 1896. Elementary expositions will be found in the two volumes of his Vorträge und Reden, Brunswick, 1884, of which there is an English translation by E. Atkinson, with the title: Popular Lectures on Scientific Subjects, 2nd ed., London, 1893. 
There are one or two questions, however, relating principally to Ohm's Law ( $\$ 1$ ), to which some reference is necessary. The first point on which the student should satisfy himself is that the various simple-harmonic vibrations which are as a rule combined in the production of a musical note are really represented by independent elements in the resulting sensation; that the latter can in fact be resolved into a fundamental tone and a series of harmonics. For this a slight course of education is necessary. A series of resonators of the type shewn in Fig. 80, p. 261, tuned to the overtones which it is desired to detect, are of great service for this purpose*. But such assistance is not indispensable, and a good deal can be effected with the piano or monochord. Take for instance the note $c$, whose harmonics are $c^{\prime}, g^{\prime}, c^{\prime \prime}, e^{\prime \prime}, g^{\prime \prime}, \ldots$ If on the piano one of these, say $g^{\prime}$, be gently sounded, and the key then released, so that the vibration is stopped, and if immediately afterwards the note $c$ be struck with full intensity, it is not difficult to recognize in the compound sensation the presence of the element previously heard. This is often more perceptible as the sound dies away, the overtones being apparently extinguished more slowly than the fundamental. A more immediately convincing series of experiments can be made with the monochord, or with a piano whose strings are horizontal and therefore easily accessible. If a string be set into vibration whilst damped at a nodal point of one of the harmonics by contact with a hair-pencil, the fundamental tone and all the harmonics of lower rank may be reduced in intensity or altogether extinguished, according to the degree and duration of the pressure applied. In this way a whole series of types of vibration can be produced in which the harmonic in question is accompanied by a varying admixture of the fundamental, \&c. The occurrence throughout of the corresponding sensation as an independent element in the resulting sound is in this way easily appreciated. The piano also lends itself readily to the

- * It may be noted that the external ear-cavity is itself a resonator, responding most intensely to a certain tone, which varies for different individuals but is usually in the neighbourhood of $e^{i v}-g^{i v}$. The aperture being relatively large, the damping and consequently the range of resonance is considerable. 
analysis of compound notes by resonance. If the string $c^{\prime}$, for example, be freed from its damper by holding down its key, whilst $c$ is sounded for a moment, the harmonic $c^{\prime}$ is taken up and continued by the first-mentioned string. If on the other hand, the string $c$ be free from its damper, whilst $c^{\prime}$ is sounded for a moment, the tone $c^{\prime}$ is taken up as a harmonic of the lower string. These simple experiments, which (with others) are recommended by Helmholtz, can of course be varied in many ways. Again, when the ear has learnt to distinguish the partial tones in a complex note, it is easy to note the absence of a particular tone of the series when the corresponding simpleharmonic vibration is not excited. For instance, when a string is struck at its middle point, the harmonics of even order are wanting ( $(26)$.

\section{Influence of Overtones on Quality.}

The quality of a musical note is determined $(\S 2)$ by the number and relative intensities of the various tones which compose it. The kind of influence which overtones of different ranks exercise on the quality is summarised by Helmholtz, somewhat as follows:

$1^{0}$. Pure tones like those of tuning forks with resonance boxes, or of wide stopped organ pipes, are soft and pleasing, smooth, but wanting in power.

$2^{0}$. Notes which contain a series of overtones up to the fifth or sixth in rank are richer and more musical, and are perfectly smooth so long as no higher overtones are sensible. The notes of the piano and of open organ pipes are examples, whilst those of the flute, and of the flute-stops on the organ when softly played, approximate more to the character of pure tones. In the "mixture" stops of the organ the lower harmonics are expressly provided in greater intensity by auxiliary pipes which are played automatically along with that which gives its name to the note.

30. When the harmonics of even order are absent, as in the case of a stopped organ pipe, or a piano string struck at the middle point, the note has a hollow, and even a nasal character, if the odd harmonics are numerous. 
4". The sound may further be described as "full," if the fundamental tone be predominant, and as "empty" if it be relatively feeble. This is exemplified in the difference of quality between the sound of a piano-wire when struck with a soft or a hard hammer, respectively $(\$ \$ 26,38)$.

$5^{\circ}$. When harmonics beyond the sixth or seventh are present in considerable intensity, the sound is harsh and rough, owing to the discords which these higher overtones make with one another. If, however, the higher harmonics, though present, are relatively weak, as in the case of the stringed instruments of the orchestra, reed-pipes, and the human voice, they are useful as giving character and expression to the sound. Brass instruments, on the other hand, with their long series of powerful overtones, are as a rule only tolerable in combination with others, or for the sake of particular effects.

The analysis of the sounds of the human voice is naturally a more difficult matter. In particular, the constitution of the vowel sounds has been much debated, without any very definite conclusion. The same vowel may be sung on a wide range of notes, but preserves its peculiar character throughout; and the question arises, does this special quality depend solely on the relative intensities of the various partial tones, or on the predominance of one or more overtones of, or near to, a particular pitch? It will be remembered that the vibration of the larynx is periodic, and that particular harmonics may be reinforced by the resonance of the mouth-cavity, as in the case of a reed-pipe $(\$ 90)$. The balance of authority appears to incline, though not very decisively, to the "fixed-pitch" theory, which is the second of the two alternatives above stated. A review of the subject down to the year 1896 will be found in the concluding chapter of Lord Rayleigh's treatise.

93. Interference of Pure Tones. Influence on the Definition of Intervals.

It has so far been assumed that the sensations due to two coexistent simple-harmonic vibrations are produced quite independently of one another. This appears to be in fact the 
case when the interval between the two tones is sufficiently great; but when the interval is small we have "interference," as we should expect from the analysis of $\S 10$, and the sensation is in whole or in part intermittent. The phenomenon of " beats" hardly needs description; it is often met with in mistuned pairs of piano-wires, in the vibrations of finger-bowls, and so on. For methodical study two pure tones are required of equal intensity, as e.g. from two tuning forks (with resonators), or two stopped organ pipes, which can be made to differ in pitch by a variable amount. As unison is departed from, the beats (whose frequency is always equal to the difference of the frequencies of the primary tones) are at first slow and easily counted. As the interval widens they become more rapid, and a sensation of roughness or discord is experienced; moreover, the primary tones are now heard along with the beats. Finally, as the interval is continually increased, the beats and the consequent roughness gradually cease to be perceptible.

The intervals at which roughness begins and ceases, vary in different parts of the scale. For the same interval the roughness is less, the higher the pitch; on the other hand for a given number of beats per second the roughness is greater in the higher octaves.

In the case of two (or more) compound musical notes, we may have beats and eventual roughness between any constituent tones which are sufficiently near in the scale. We may even have interference between the higher overtones of the same note; and it is for this reason that harmonics of higher order than the sixth are prejudicial to good musical quality.

It is through the interference of pairs of overtones that deviations from the consonant intervals ( $\$ 3$ ) usually make themselves felt. Thus in the case of the Octave $c c^{\prime}$ we have tones with the frequencies

$$
\begin{aligned}
& c=132, \quad 264, \quad 396, \quad 528, \quad 660, \quad 792, \ldots \text {, } \\
& c^{\prime}=\quad 264, \quad 528, \quad 792, \ldots \text {, }
\end{aligned}
$$

and if this be mistuned all the harmonics of $c^{\prime}$ are interfered with by the even harmonics of $c$. 
In the case of the Fifth $c g$ we have

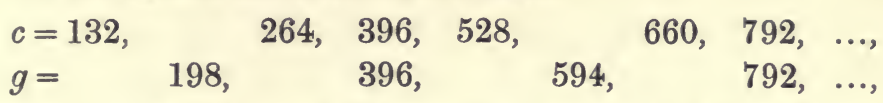

and if this be mistuned the second tone of $g$ beats with the third tone of $c$, and so on. When the ratio of the vibration numbers of the fundamentals is less simple, the harmonics which can interfere are of higher order. Thus in the case of the Major Third, where the ratio is $4: 5$, the first pair of interfering overtones consists of the fifth tone of the lower note, and the fourth of the higher. Since in many musical instruments the fifth tone is very feeble, this consonance is less well defined than the preceding ones. On the other hand the fundamentals may fall, in the lower parts of the scale, within beating distance (for example $c=132, e=165$ ), so that this consonance is to be reckoned also as less perfect than the former ones. Similar remarks apply with greater force to such cases as the Minor Third $(5: 6)$ and the Minor Sixth $(5: 8)$.

\section{Helmholtz Theory of Audition.}

The connection between primary sensations and simpleharmonic vibrations has still to be accounted for. The problem is a physiological one; but the theory which Helmholtz has framed to explain Ohm's law, so far as it holds, and the various deviations from it, is in its essentials so simple, and is so successful in binding together the facts of audition into a coherent system, that a brief statement of it may be attempted.

In its simplest form the theory postulates the existence, somewhere in the internal ear, of a series of structures each of which has a natural period of vibration, and is connected with a distinct nerve-ending. For brevity we will speak of these structures as "resonators," since that is their proper function. A particular resonator is excited whenever a vibration of suitable frequency impinges on the ear; the appropriate nerve is stimulated; and the sensation is communicated to the brain. In this way the resolution of a musical note into its constituent tones is at once accounted for.

It is necessary to suppose that the resonators are subject 
to a considerable amount of damping. If it were not so, each resonator would go on vibrating, and the corresponding sensation would persist, for an appreciable time after the exciting cause had ceased. A similar interval of time would elapse before the sensation reached its full intensity when the cause first sets in. The effect would be that the sensations due to a sufficiently rapid succession of distinct notes would not be altogether detached from one another in point of time. From considerations of this kind Helmholtz estimated that the degree of damping must be such that the intensity (as measured by the energy) of a free vibration would sink to one-tenth of its initial value in about ten complete vibrations. It follows, as explained in $\S 13$, that each resonator will respond to a certain range of frequencies on each side of the one which has maximum effect. It is assumed, further, that the difference of pitch of adjacent resonators is so small that the same simple-harmonic vibration will excite a whole group, the intensity falling off from the centre on either side.

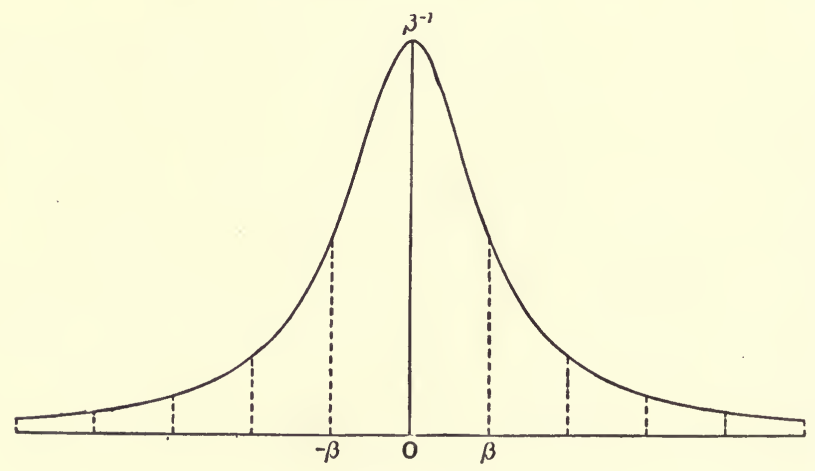

Fig. 85 .

This is illustrated by the annexed figure, repeated from $\S 13$, which may now serve to exhibit the distribution of intensity over a continuous series of resonators under the influence of a given simple-harmonic vibration. The abscissa is $p / n-1$, where $p$ is now taken to represent the natural frequency of a resonator, and $n$ that of the imposed vibration. The horizontal scale depends on the value of $\beta$, or $1 / n \tau$, where $\tau$ is the 
modulus of decay of a free vibration. On the above estimate of Helmholtz we shall have

$$
e^{-40 \pi / n \tau}=\frac{1}{10},
$$

whence $\beta=\cdot 018$. The intensity is therefore one-half the maximum for

$$
\frac{p}{n}=1 \pm \cdot 018
$$

It will be observed that on the above view we ought in strictness to speak of "simplest" rather than of "simple" sensations of sound, absolutely simple sensations, in the strict physiological meaning, being impossible to excite.

When two simple-harmonic vibrations, sufficiently far apart in the scale, are in operation, the two groups of resonators which are affected will be practically independent, and the two sensations (of pure tones) will coexist. But when the interval between the frequencies is sufficiently small, the two groups will overlap, and the energy of vibration of those resonators which are common to them will fluctuate in the manner explained in $\$ 10$. The excitation of the corresponding nerve-endings will therefore be intermittent, with a frequency equal to the difference of those of the two originating vibrations. This is, on the theory, the explanation of beats. As the interval is increased, the beats become more rapid. The "roughness" which is ultimately perceived, in spite of the diminishing amplitude of the fluctuations, has a more remote physiological explanation. According to Helmholtz, there is here an analogy with the painful effect produced by a flickering light, and in other cases where a nerve is stimulated repeatedly at intervals of time which are neither too great nor too small. When the intervals are sufficiently long, the nerve has time to recover its initial sensibility, and so experiences the full effect of each recurring stimulus. When on the other hand the intervals are sufficiently short, the sensation tends to become continuous. It is for this reason that beats exceeding, say, 132 per second cease to produce the sensation of roughness, even although the interval between the beating tones be such as would be perceptibly discordant in a lower part of the scale. 
The student of dynamics cannot fail to admire the beauty of a theory which lends itself readily to the explanation of so many complicated relations; but it is with the physiologist and the anatomist that in the last resort it lies to decide whether a mechanism of the kind postulated is really to be found in the internal ear. In the original form of the theory (1862) the resonators were identified with the structures known as "Corti's rods," which are found arranged, some 3000 in number, along the basilar membrane in the spiral cavity of the cochlea. A disturbing discovery by Hasse that these structures do not occur in the ears of birds, to whom we can hardly deny the perception of pitch, led to a modified form of the theory. In the third edition of the Tonempfindungen (1870) Helmholtz propounded the view that the resonators consist of the various parts of the basilar membrane itself. This membrane varies in breadth from one end to another, like a very acute-angled triangle, and the tension appears to be very much less in the direction of length than in that of breadth. On this view the different parts could be set into sympathetic vibration, much as in the case of a series of strings of variable length placed side by side, except that the independence of adjacent parts would be approximate instead of absolute. For a full description of the complicated structure of the internal ear, and for further speculations as to the functions performed by its various parts, we must refer to books on physiology.

\section{Combination-Tones.}

In one important respect the theory as so far developed is inadequate. The explanation of consonant intervals outlined in $\S 93$ assumes that one at least, and generally both, of the notes concerned is complex, and contains one or more overtones in addition to the fundamental. It was in fact through the interference of two tones, one at least of which is an overtone, that departure from the exact relation of pitch was stated to make itself manifest. When both tones are pure this means of definition is wanting, and on the theory of audition sketched in the preceding section there appears to be no reason why 
the octave (for example) should be distinguished by any character of smoothness from adjacent intervals on either side, the two groups of sensations being in any case quite independent. Since the more consonant intervals at all events are as a matter of fact easily recognized by the ear, even in the case of apparently pure tones, and are thoroughly well defined, the difficulty is a serious one. To meet it, Helmholtz developed his theory of "combination-tones," which are assumed to supply the function of the missing overtones.

In most of our investigations it has been assumed that the amplitude of the vibrations may be treated as infinitely small, so that disturbances due to different sources may be superposed by mere addition. In the theory now in question this assumption is abandoned; the vibrations are regarded as small, but not as infinitely small, and the interaction of the disturbances due to different causes is, to a certain degree of approximation, investigated.

We have already had an indication in $\S 63$ of the manner in which two imposed simple-harmonic disturbing forces of small but finite amplitude, with frequencies $N_{1}, N_{2}$ respectively, may generate in the air other simple-harmonic vibrations whose frequencies are

$$
2 N_{1}, \quad 2 N_{2}, \quad N_{1}-N_{2}, \quad N_{1}+N_{2},
$$

and whose amplitudes involve the squares or product of the amplitudes of the two primaries. If the approximation were continued we should meet with further vibrations whose frequencies are of the type $p_{1} N_{1} \pm p_{2}{ }^{\circ} N_{2}$, where $p_{1}, p_{2}$ are integers. In acoustical language, two simple-harmonic vibrations can, if of sufficient intensity, give rise not only to the pure tones usually associated with them, but also to a series of other pure tones of higher order. The fact that a single harmonic vibration can by itself give rise to a pure tone together with its octave, \&c. is itself of some importance, but the most interesting result is due to the interaction, viz. the "difference-tone" $\left(N_{1}-N_{2}\right)$. The existence of difference-tones was observed, apart from all theory, by Sorge (1745) and Tartini (1754). The "summation-tone" $\left(N_{1}+N_{2}\right)$ is more difficult to hear, and its 
existence has even been denied. It has however been objectively demonstrated by Rücker and Edser*, by its effect on a tuning fork of the same frequency.

Difference-tones due to the causes just considered are most easily perceptible where we have a mass of air which is subject to the joint and vigorous action of the primary vibrations, as in the harmonium and the siren; they can then, like other tones, be reinforced by suitable resonators.

There is however a way in which combination tones may conceivably be originated in the ear itself. To explain this it is necessary briefly to consider the forced vibrations of an unsymmetrical system. When a particle, or any system having virtually one degree of freedom, receives a displacement $x$, the force (intrinsic to the system) which tends to restore equilibrium is a function of $x$, and may be supposed expressed, for small values of $x$, by a series

$$
\mu x+\alpha x^{2}+\beta x^{3}+\ldots
$$

An example is furnished by the common pendulum, where the force of restitution is proportional to $g \sin \theta$, or

$$
g \theta-\frac{1}{6} g \theta^{3}+\frac{1}{120} g \theta^{5}-\ldots \text {; }
$$

but here, on account of the symmetry with respect to the vertical, the force changes $\operatorname{sign}$ with $\theta$, so that only odd powers of $\theta$ occur. The correction for small finite amplitudes depends therefore on the term of the third order in $\theta$. But if the system be unsymmetrical, as in the case of a pendulum hanging from the circumference of a horizontal cylinder $\dagger$, the term of the second order comes in, and the correction is more important. Helmholtz lays stress on the fact that in the slightly

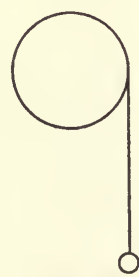

Fig. 86.

* Phil. Mag. (5), vol. xxxrx. (1895).

† If $a$ be the radius, and $l$ the length of the free portion of the string when vertical, the potential energy is

$$
V=\frac{1}{2} \frac{m g}{l} s^{2}-\frac{1}{6} \frac{m g a}{l^{3}} s^{3}+\ldots,
$$

where $s$ is the are described by the bob from the lowest position. The restoring force is therefore

$$
\frac{d V}{d s}=\frac{m g}{l} s-\frac{1}{2} \frac{m g a}{l^{3}} s^{2}+\ldots
$$


funnel-shaped tympanic membrane and its connections we have precisely such an unsymmetrical system, the restoring force being somewhat greater for inward than for outward displacements of the same extent*. If we keep only the first two terms in (1), the equation of motion is of the type

$$
\ddot{x}+\mu x=-\alpha x^{2}+X,
$$

where $X$ represents the disturbing forcet. The joint action of two simple-harmonic forces will be represented by

$$
X=f_{1} \cos n_{1} t+f_{2} \cos n_{2} t .
$$

Neglecting, for a first approximation, the square of $x$, we have

$$
x=\frac{f_{1}}{\mu-n_{1}{ }^{2}} \cos n_{1} t+\frac{f_{2}}{\mu-n_{2}{ }^{2}} \cos n_{2} t,
$$

the terms which represent the free vibrations being omitted, since these are rapidly destroyed by dissipation. If we substitute this value of $x$ on the right hand of (2), and write for shortness

$$
f_{1} /\left(\mu-n_{1}^{2}\right)=g_{1}, \quad f_{2} /\left(\mu-n_{2}^{2}\right)=g_{2},
$$

we obtain the differential equation

$$
\begin{aligned}
\ddot{x}+\mu x=X & -\frac{1}{2} \alpha\left(g_{1}^{2}+g_{2}^{2}\right)-\frac{1}{2} \alpha g_{1}^{2} \cos 2 n_{1} t-\frac{1}{2} \alpha g_{2}^{2} \cos 2 n_{2} t \\
& -\alpha g_{1} g_{2} \cos \left(n_{1}-n_{2}\right) t-\alpha g_{1} g_{2} \cos \left(n_{1}+n_{2}\right) t, \ldots
\end{aligned}
$$

correct to the second order of $f_{1}, f_{2}$. The terms written in full on the right hand may be regarded as a correction to the disturbing force $X$. The solution of (6) gives, in addition to (4), the terms

$$
\begin{aligned}
& -\frac{1}{2} \frac{\alpha}{\mu}\left(g_{1}^{2}+g_{2}^{2}\right)-\frac{1}{2} \frac{\alpha g_{1}^{2}}{\mu-4 n_{1}^{2}} \cos 2 n_{1} t-\frac{1}{2} \frac{\alpha g_{2}{ }^{2}}{\mu-4 n_{2}^{2}} \cos 2 n_{2} t \\
& -\frac{\alpha g_{1} g_{2}}{\mu-\left(n_{1}-n_{2}\right)^{2}} \cos \left(n_{1}-n_{2}\right) t-\frac{\alpha g_{1} g_{2}}{\mu-\left(n_{1}+n_{2}\right)^{2}} \cos \left(n_{1}+n_{2}\right) t .
\end{aligned}
$$

The first term merely indicates a shift of the mean position

* It may be noted that the same element of asymmetry is present in the investigation of $\S 63$. When we proceed to the second order of small quantities, the changes of pressure due to condensations $\pm s$ are no longer equal in amount.

+ It is unnecessary to take account of the variability of inertia, since this can be got rid of by a proper choice of the coordinate $x$. In any case it will not alter the general character of the results obtained in the second approximation. 
about which the oscillations take place. For the rest, we have octaves of the primary tones, together with a difference- and a summation-tone. If the approximation were continued we should obtain combination-tones of higher order, as in the former case.

When, as in the case of the tympanic membrane, the free period $2 \pi / \sqrt{ } \mu$ is relatively long, the most important combination-tone is the difference-tone $\left(n_{1}-n_{2}\right)$, on account of the relative smallness of the corresponding denominator in (7).

The theory of combination-tones here reproduced has not been accepted without question. The difference-tones, as already mentioned, were known as a fact since the time of Tartini, and a plausible explanation had been given by Thomas Young (1800). According to this view the beats between the two tones, as the interval increases, ultimately blend, as if they were so many separate impulses, into a continuous tone having the frequency of the beats. The difficulty of this explanation is that the actual impulses during a beat are as much positive as negative, so that it does not appear how any appreciable residual effect in either direction could be produced, if the vibrating system be symmetrical. It is true that if we turn to the figure on p. 23, it is apparently periodic, with the period of the intermittence; but from the point of view of Fourier's theorem the lower harmonics are all wanting, and the only two which are present are precisely the two which are used in constructing the figure. On the Helmholtz theory of audition the intermittent excitation of a particular resonator $m$ times a second is a wholly different phenomenon from the excitation of an altogether distinct resonator whose natural frequency is $m$. Young's view appears indeed to be inadmissible on any dynamical theory of audition, at least in the case of infinitely small vibrations. On the other hand it is true, as we have seen, that given a finite amplitude, and an unsymmetrical system, a vibration of the type shewn in Fig. 10, p. 23, does actually generate (among others) a vibration whose period corresponds to the fluctuations there shewn. The distinction between the two theories might therefore, from a merely practical point of 
view, be held to be almost verbal, were it not that Young's theory fails to give an explanation of combination-tones other than the first difference-tone.

96. Influence of Combination-Tones on Musical Intervals.

A brief indication of the way in which combination-tones may assist in defining the consonant intervals is all that can be attempted here. Take first the case of (primarily) pure tones. In the case of a slightly mistuned Octave, say $N_{1}=100$, $N_{2}=201$, we have $N_{2}-N_{1}=101$, which gives a difference-tone making 1 beat per second with $N_{1}$.

For the Fifth, let $N_{1}=200, N_{2}=301$. We have

$$
N_{2}-N_{1}=101,2 N_{1}-N_{2}=99 \text {, }
$$

giving combination-tones with 2 beats per second.

For the Fourth, let $N_{1}=300, N_{2}=401$. Then

$$
2 N_{1}-N_{2}=199,2 N_{2}-2 N_{1}=202,
$$

and the corresponding tones make 3 beats per second.

For the Major Third, let $N_{1}=400, N_{2}=501$. We have $2 N_{2}-2 N_{1}=202,3 N_{1}-2 N_{2}=198$, giving 4 beats per second.

We might proceed further in the list, but it will already have been remarked that combination-tones of increasingly high order are being invoked. This is quite in conformity with the observed fact that the beats are, in all cases after the octave, very faint unless the primaries be especially vigorous.

A more effective part is played by the combination-tones when the notes concerned have one or two overtones, but not a sufficient range of them to account for the definition on the principles of $\S 93$. Take for instance the case of the Fifth, when each note has a first harmonic in addition to the fundamental. If the interval be slightly mistuned, we have say the primary tones: 200,$400 ; 301,602$. These give the two difference-tones $301-200=101,400-301=99$, which interfere with one another.

The combination-tones have an influence again, in the case 
of consonant triads, especially of simple tones, but enough has been said to shew their importance from the musical point of view. For further developments reference must be made to the work of Helmholtz*.

\section{Perception of Direction of Sound.}

One important question of physiological acoustics in which dynamical principles are involved remains to be mentioned. An observer, even when blindfolded, and with no adventitious circumstances to guide him, is in general able to indicate with great accuracy the direction from which a sound proceeds. In the case of pure tones the discrimination between back and front is indeed lost, as was to be expected, considering the symmetry with respect to the medial plane of the head, but right and left are clearly distinguished. For tones of small wave-length this may be accounted for by the difference of intensity of the sensation in the two ears, since the head acts to some extent as a screen, as regards the further ear. But when the wave-length of the sound much exceeds the perimeter of the head the investigation given near the end of $\S 81$ shews that this difference must be very slight. According to the most recent investigations of Lord Rayleigh + , the interpretation depends on the relative phase of the sounds as they. reach the two ears, a difference of even a fraction of a period being effective. He found that if the same tone be led by different channels to the two ears, and all extraneous disturbances be excluded, the sound can be made to appear to come from the right or left, at will, by adjusting the relative phase. The origin of the sound was always attributed to that side on which the phase is in advance (by less than half a period). The result, which has been arrived at independently by other observers, is at present unexplained. It has been suggested that the phenomena may really be due to a difference of intensity. A fraction of the sound may be transmitted from each side to the opposite internal ear, through the bones of

* See also Sedley Taylor, Sound and Music, London, 1873.

† Phil. Mag. (6), vol. xiı. (1907). 
the head, in which case the original difference of phase would produce a slight difference of intensity on the two sides owing to interference between the direct and transmitted vibrations*. It is impossible to suppose, however, that this difference could be other than exceedingly minute.

* Myers and Wilson, Proc. Roy. Soc. vol. Lxxx. A, p. 260 (1908). This hypothesis is discussed by Lord Rayleigh, Proc. Roy. Soc. vol. LxxxiI. A, p. 61 (1909). 



\section{INDEX}

\section{[The numerals refer to the pages]}

Absorption of sound, 196

Adiabatic lines, 158

Air-waves, general theory of, 204 see also Sound waves

Amplitude, minimum audible, 167

Analysis of sound sensations, 2, 284

Anticlastic curvature of a flat bar, 152

Approximate solution of periodequations, $83,125,126,128,256$, 258

Audibility, range of frequency for, 3 least amplitude for, 167

Audition, Helmholtz theory of, 289

Bars, longitudinal vibrations, 114 flexural vibrations, 120,130

Beats, 23, 132, 138 relation of, to dissonance, 288

Bells, 155

Bessel's functions, 85, 145, 147, 259

Blackburn's pendulum, 35

Chain, vibrations of hanging, 84

Circular vibrations, 55

'Circulation' defined, 203 •

Clamped-free bar, transverse vibrations of $\mathrm{a}, 127$

Combination-tones, 181, 292, 294!

Communication of vibrations to a gas, 237

'Condensation' defined, 160

Conduction of heat, effect of, on sound waves, 187

'Conductivity' of an aperture, 245

Conical pipe, normal modes of a, 257

Consonant intervals, 3, 283, 297

Cosine-series, 92

Curved shells, vibrations of, 155

Cylindrical vessel, normal modes of a, 259

Damping of vibrations, $25,27,57$

effect of, on resonance, 32

of air-waves by viscosity, 185, 186, 190

of a resonator, 265

of an organ pipe, 269
Degrees of freedom of a dynamical system, 12,14

Diatonic scale, 5

Diffraction of sound, 240, 244, 248

'Dilatation' defined, 107

Direction of sound, perception of, 298

Discontinuity, waves of, 181

Dissipation of energy by friction, 27, 184

Dissipation (apparent), by generation of air-waves, 166, 225, 227, 232, 264, 269

'Divergence' defined, 199

Doppler's principle, 221

Double pendulum, 38

'Double source' of sound, 215, 226

Elasticity, elementary theory of, 106 coefficients of, 110, 113 of gases, 159

Elliptic vibrations, 49

Emission of energy, by a simple source, 225

by a double source, 227

by a resonator, 265

by an open pipe, 269

Energy, of a simple-harmonic vibration, 15

of a string, 60

of an elastio solid, 114

of a bar, 123

of a membrane, 141

of a bent plate, 151

of air-waves, 163, 205

'Extension' defined, 107

Extensional vibrations of a rod, 114 of a circular ring, 136

Finite amplitude, air-waves of, 174

Flexure, uniform, of a bar, 121 of a plate, 150

Flexural vibrations, of a bar, 120 , of a ring, 136

of a plate, 153

'Flux,' defined, 199

'Flux of energy,' 165, 224 


\section{[The numerals refer to the pages]}

Forced oscillations, 16, 20, 47 effect of friction on, 28, 57, 104

Fork, tuning, 132

Fourier's theorem, 87, 92 influence of discontinuities in, 92 law of convergence of coefficients in, 94

Freedom, degrees of, 12, 34

Free-free bar, transverse vibrations of a, 124

Free oscillations, 12 with friction, 24 general theory of, 44

Frequency, range of, for audibility, 3

Friction. See Dissipation

Gas, elasticity of a, 159 isothermal and adiabatic lines of a, 157,158

Graphical solution of period-equations, $83,125,126,128,256,258$

Grating, transmission of sound by a, 247

Harmonic analysis, 101

Harmonics, 5

Heat, vibrations caused by, 278

Heat-conduction, effect of, on sound waves, 187

Hooke's law of elasticity, 11, 110

Huygens' principle, 249

Imaginaries, use of, 53

Impact, vibrations of a string due to, 73,99

Indicator diagram, 157

Inertia, coefficients of, 42

'Irrotational ' motion defined, 203

Interference of simple-harmonic vibrations, 23

of pure tones, 287

Intervals, musical, 5, 288 degree of definition of, 297

Isothermal lines, 157

Laplace's equation, 205

Leslie's experiment, 237

Lines of motion, 229

Lissajous' figures, 49

Loaded string, normal modes of a, $\mathbf{3 6}$, 37,82

Local periodic force, effect of, in a gaseous medium, 233

Longitudinal vibrations, of bars, 114 of columns of air, 170, 266

Loops, on a vibrating string, 70 in a pipe, 171
Membrane, transverse vibrations of a, 139

normal modes of a rectangular, 142

of a circular, 144

Mersenne's laws, 70

'Modulus of decay,' defined, 25

of air-waves, 185

of a vibrating sphere, 233

of a resonator, 265

of a pipe, 270

Modulus, Young's, 111

Multiple system, equations of motion of a, 41,44

normal modes of a, 44

forced vibrations of $a, 47$

Nodal lines, of a membrane, 143, 144, 148

of a plate, 153,154

Nodes, in a vibrating string, 70

in a bar, 116, 127

in a pipe, 171

' Normal functions,' 101, 130

Normal modes of vibration, 44

Notes, musical, 3, 285

Ohm's law, 2, 285, 289

Organ pipe, normal modes of, 171 corrected theory of, 266

mode of action of, 276

Overtones, 5

influence of, on quality, 286

on the definition of consonant intervals, 288

Pendulum, 8, 16

Blackburn's, 35

double, 38

Period-equations, graphical solution of, $83,125,126,128,256,258$

Permanency of type, condition for, in air-waves, 174

Pipe, normal modes of a, 171 modulus of decay of a, 270 velocity of sound in a narrow, 193

Plane waves in an elastic medium, 118 in air, 160, 174, 223

Plate, transverse vibrations of a circular, 153 ; of a square, 154

Plucked string, theory of, 66,98

Poiseuille's law, 195

Poisson's ratio, 111

'Quality' of musical notes, 4 influence of overtones on, 286

Reciprocity, principle of, 47, 81 
[The numerals refer to the pages]

Rectangular vessel, normal modes of a, 254

Reed-pipes, theory of, 278

Reflection of waves, $64,168,215$, 267

Refraction of sound, due to variation of temperature, 216

to wind, 219

Resonance, 18, 20, 22, 32, 270

Resonator, 261

free vibrations of a, 262

forced vibrations of a, 270

Ring, normal modes of a, 133

Scattering of sound waves by obstacles, 240

Sensations, analysis of, 2,284

Shearing strain, 107

stress, 109

Shells, vibrations of curved, 155

'Simple source' of sound, 214,224

Simple-harmonic vibrations, 2, 9

energy of, 15

superposition of, 22,48

Sine-series, 87

Sound, velocity of, in air, 161, 162 in water, 163

Sound waves, plane, 160

spherical, 205, 224

general, 204, 212, 214

of finite amplitude, 174

Sounding board, function of, 68,81

Source of sound, simple, 214, 224 double, 215,226

'Speed' of a simple vibration, 10

Sphere, waves produced by oscillating, 228

vibrations of an elastic, 156

Spherical vessel, normal modes of a, 255,258

Stability, coefficients of, 43

Stationary property of normal modes, 45

Stiffness of piano-wire, effect of, 82, 133

Strains, 106

Stresses, 108
String, transverse vibrations of a, 59 waves on a, 61,64 normal modes of a finite, 68

forced vibrations of $a, 80$

String excited by plucking, 66, 72, 98

by impact 73,99

by bowing, $7 \overline{5}, 98$

Superposition of vibrations, 22, 48

Temperament, equal, 7

Temperature, effect of unequal, on propagation of sound, 216

Tension, effect of permanent, on the vibrations of a bar, 132

Tones, pure, 1 interference of, 287

Transmission of sound by an aperture, 244

by a grating, 247

Transverse vibrations, of strings, 59

of bars, 120

of membranes, 139

of plates, 152

Tuning fork, 132

Velocity of sound, 161, 162

in a narrow pipe, 193

-Velocity-potential,' 201

Violin-string, 75, 98

Viscosity, 183

effect of, on air-waves, 185, 186, 190

on waves in a narrow pipe, 193, 194, 196

Water, velocity of sonnd in, 163 vibrations of a column of, 173

Watt's indicator diagram, 157

Waves, on a string, 61,64

in a bar, 115,123

in an elastic medium, 118. See also Sound waves

Wind, influence of, on sound propagation, 219

Young's modulus, 111 




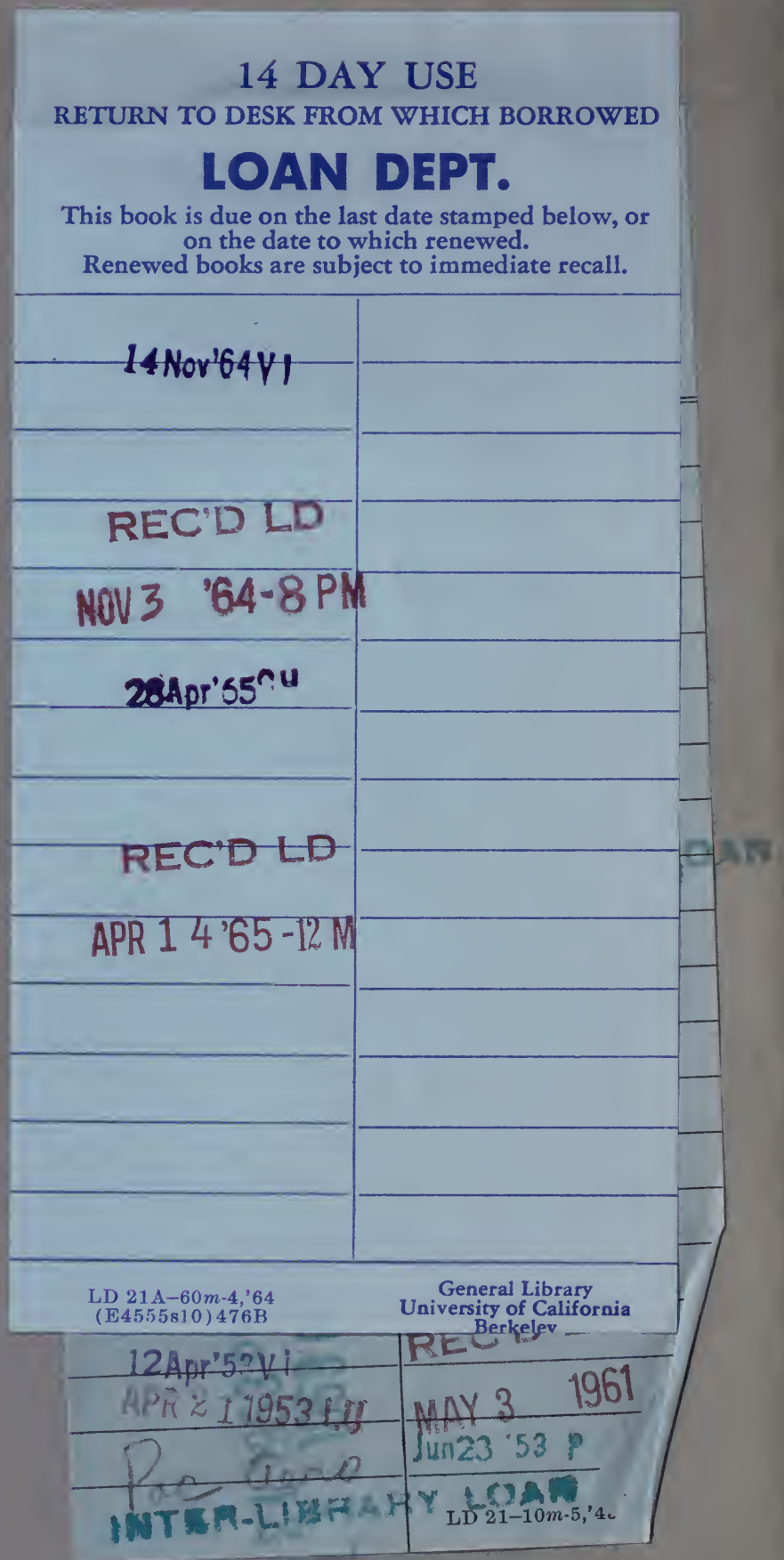


YC 10940

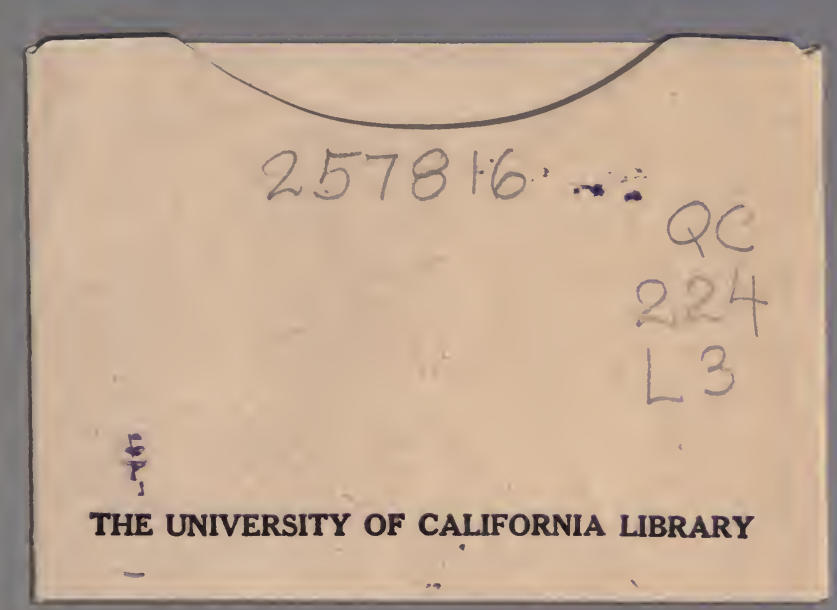


$y-x=$ 